This item was submitted to Loughborough's Research Repository by the author.

Items in Figshare are protected by copyright, with all rights reserved, unless otherwise indicated.

\title{
Design and additive manufacture for flow chemistry
}

PLEASE CITE THE PUBLISHED VERSION

\section{PUBLISHER}

(c) Andrew James Capel

\section{PUBLISHER STATEMENT}

This work is made available according to the conditions of the Creative Commons Attribution-NonCommercialNoDerivatives 4.0 International (CC BY-NC-ND 4.0) licence. Full details of this licence are available at: https://creativecommons.org/licenses/by-nc-nd/4.0/

\section{LICENCE}

CC BY-NC-ND 4.0

\section{REPOSITORY RECORD}

Capel, Andrew J.. 2019. "Design and Additive Manufacture for Flow Chemistry". figshare. https://hdl.handle.net/2134/21613. 
A thesis submitted in partial fulfilment of the requirements for the award of Doctor of Philosophy

\title{
Design and Additive Manufacture for Flow Chemistry
}

\author{
Andrew James Capel
}

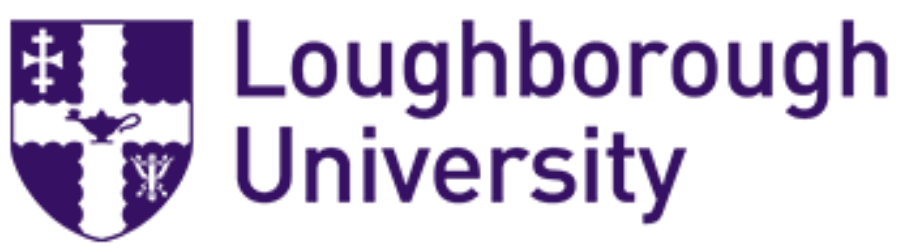

Supervisors: Dr S.D.R Christie, Dr S. Edmondson, Dr R.D Goodridge

(C)Andrew James Capel 2015 


\section{Certificate of Originality \\ Thesis Access Conditions and Deposit Agreement}

Students should consult the guidance notes on the electronic thesis deposit and the access conditions in the University's Code of Practice on Research Degree Programmes

Author: Andrew James Capel

Title: Design and Additive Manufacture for Flow Chemistry

I [Andrew James Capel], "the Depositor",

Would like to deposit [Design and Additive Manufacture for Flow Chemistry], hereafter referred to as the "Work", once it has successfully been examined in Loughborough University Institutional Repository

Status of access OPEN / RESTRICTED / CONFIDENTIAL

Moratorium

Period. years,

ending 20.

Status of access approved by (CAPITALS):

Supervisor (Signature)

School of Science

Author's Declaration I confirm the following:

\section{CERTIFICATE OF ORIGINALITY}

This is to certify that I am responsible for the work submitted in this thesis, that the original work is my own except as specified in acknowledgements or in footnotes, and that neither the thesis nor the original work therein has been submitted to this or any other institution for a degree

\section{NON-EXCLUSIVE RIGHTS}

The licence rights granted to Loughborough University Institutional Repository through this agreement are entirely non-exclusive and royalty free. I am free to publish the Work in its present version or future versions elsewhere. I agree that Loughborough University Institutional Repository administrators or any third party with whom Loughborough University Institutional Repository has an agreement to do so may, without changing content, convert the Work to any medium or format for the purpose of future preservation and accessibility.

\section{DEPOSIT IN LOUGHBOROUGH UNIVERSITY INSTITUTIONAL REPOSITORY}

I understand that open access work deposited in Loughborough University Institutional Repository will be accessible to a wide variety of people and institutions - including automated agents - via the World Wide Web. An electronic copy of my thesis may also be included in the British Library Electronic Theses On-line System (EThOS). 
I understand that once the Work is deposited, a citation to the Work will always remain visible. Removal of the Work can be made after discussion with Loughborough University Institutional Repository, who shall make best efforts to ensure removal of the Work from any third party with whom Loughborough University Institutional Repository has an agreement. Restricted or Confidential access material will not be available on the World Wide Web until the moratorium period has expired.

- That I am the author of the Work and have the authority to make this agreement and to hereby give Loughborough University Institutional Repository administrators the right to make available the Work in the way described above.

- That I have exercised reasonable care to ensure that the Work is original, and does not to the best of my knowledge break any UK law or infringe any third party's copyright or other Intellectual Property Right. I have read the University's guidance on third party copyright material in theses.

- The administrators of Loughborough University Institutional Repository do not hold any obligation to take legal action on behalf of the Depositor, or other rights holders, in the event of breach of Intellectual Property Rights, or any other right, in the material deposited.

The statement below shall apply to ALL copies:

This copy has been supplied on the understanding that it is copyright material and that no quotation from the thesis may be published without proper acknowledgement.

Restricted/confidential work: All access and any copying shall be strictly subject to written permission from the University Dean of School and any external sponsor, if any.

Author's

signature

..Date

user's declaration: for signature during any Moratorium period (Not Open work):

I undertake to uphold the above conditions:

\begin{tabular}{|l|l|l|l|}
\hline Date & Name (CAPITALS) & Signature & Address \\
\hline & & & \\
\hline & & & \\
\hline & & & \\
\hline & & & \\
\hline
\end{tabular}




\section{Acknowledgements}

Firstly I would like to thank my three supervisors for the fantastic support they have given me during my thesis. Ruth whose help and guidance never wavered despite moving universities and starting a family. Steve E. whose endless list of brilliant (for the most part) ideas and boundless enthusiasm helped really drive the project forward, again despite moving Universities (was it me?). And finally Steve Christie, who has shown immense trust in me over the last few years. Without your help I would not be where I am today. I never did find out what was up your kilt though! Special mention must also go to Russ Harris and the other members of the AM department, who allowed this research to progress at Loughborough after the move to Nottingham. Also thanks to Nick, David and the other members of the radiochemistry department, who put up with me squatting in their office for the last few years. And finally thanks go to THE Alistair Daley, you have filled my brain full of hours of useless trivia I will probably never forget.

A big part of my PhD was the world renowned Acrylate Stanley football team: Bull, Carlos, Wood, Jimmy, Jesus, Ben, Marc, Joel, Phil, Alvaro, Red Ranger, Sam, TC, Noble, Mike, Anish, Swaggy D and anyone else who made cameos. We never did manage to bring home the ultimate prize of the staff league, but we gave it a bloody good go. I think we can all agree with Andrew Wood that it was probably all Jimmy's fault. Thanks must also go to all the other members of the organic labs who made working there for three years bearable.

Also thanks should go to the guys who have put up with me outside of the lab. Mr (you're not a doctor yet?) Kimber, I will finally concede you may be a slightly better organic chemist than me! We have shared many memorable nights out together but your stag do was pretty unbeatable and to this day I will never know how we fitted you inside that fold up bed. Thanks to the Boston shipment (I guess) for allowing me to watch you eat so many revolting things. You were much better at those challenges than the trebuchet one which you sucked at. I'm always a graceful winner. You still owe me a 12" Christmas cake though. To Daz Valance, thanks for teaching me to become the world class fisherman I am today. The legend of Papa Roach will live on. I tried to mould you into the friend I always wanted but you constantly let me down. Seriously, when are you going to take me star gazing! I have decided to issue you with a lifetime zoo ban. Sarah I officially pass complete responsibility of him over to you, make sure you regularly groom him and check for ticks. Jimmy, I am sorry for peppering you over and over again with my booming serves. Your racquets took some abuse. 
I also apologise for some of the slightly exaggerated stories that I leaked to the Stanley press about you. The drunken behaviour and one night stands will always be remembered. Sorry Meg that you had to find out like this. And finally to Tash whose constant supplies of sweeties and gossip livened up my days. You still never ate my beef chewing gum though!

Finally thanks go my parents, whose support through the last seven years of student life have finally paid off. And most importantly to Natalie, I will always remain grateful that I have you in my life. 


\section{Abstract}

This thesis aims to investigate the use of additive manufacturing (AM) as a novel manufacturing process for the production of milli-scale chemical reaction systems. Five well developed additive manufacturing techniques; stereolithography (SL), selective laser melting (SLM), fused deposition modelling (FDM), ultrasonic additive manufacture (UAM) and selective laser sintering (SLS) were used to manufacture a number of miniaturised flow devices which were tested using a range of organic and inorganic reactions.

SL was used to manufacture a range of functioning milli-scale flow devices from Accura 60 photoresin, with both simple and complex internal channel networks. These devices were used to perform a range of organic and inorganic reactions, including aldehyde and ketone functional group interconversions. Conversion of products within these reactors, were shown to be comparable to commercially available milli-scale coil reactors. More complex designs, which allowed SL parts to be integrated to existing flow and analytical instrumentation, allowed us to develop an automated reaction analysis and optimisation platform. This platform allowed precise control over the reaction conditions, including flow rate, temperature and reagent composition. We also designed a simplex type reaction optimisation software package that could input data in the form of reaction conversions, peak intensities, and thermocouple data, and generate a new set of optimal reaction conditions. SL parts which incorporated embedded analytical components were also manufactured, which allowed us to perform inline reaction analysis as a feedback method for input into the optimisation platform. Stereolithography was shown to be a highly versatile manufacturing method for designing and producing these flow devices, however the process was shown to be still limited by the range of processable materials currently commercially available.

SLM was also used to manufacture a number of functioning milli-scale flow devices from stainless steel and titanium, which had simplistic internal channel designs of diameters ranging from 1 to $3 \mathrm{~mm}$. Again, SLM parts were manufactured which incorporated embedded analytical components, which could be integrated into an automated reaction platform. These devices, unlike parts produced via SL, could be attached to heating platforms to allow us to perform high temperature reactions. This control over the reaction temperature formed an essential part of the reaction optimisation platform. These parts were again used to perform a ketone functional group interconversion. Internal structures of these SLM parts were also 
visualised via micro computed tomography ( $\mu \mathrm{CT}$ or microCT) scanning as well as optical microscopy.

FDM was used throughout the project as an inexpensive method of prototyping parts which were to be manufactured via more expensive manufacturing processes. This prototyping allowed the optimisation of intricate design features, such as the manufacture of an inline spectroscopic flow cell for integration with a commercially available LC system. FDM was also proposed as a customisable approach to designing and manufacturing flow devices with embedded components, however the current limitations in build resolution and materials choices severely limited the use of FDM for this application.

UAM was also proposed as a novel manufacturing process whereby the build process would allow discrete components to be embedded directly into a flow channel. This was demonstrated by embedding a type-k thermocouple across a $2 \mathrm{~mm}$ channel. The data from this thermocouple was monitored during a heated reaction, and used as a method of determining the exact reaction conditions the reaction medium was being exposed to.

SLS was also proposed as a possible manufacturing method for milli-scale flow devices, however it proved difficult to remove un-sintered powder from parts with internal channel diameters as high as $5 \mathrm{~mm}$. It was shown that this powder was forming a dense semi solid, due to the large degree of shrinkage upon cooling of the SLS parts, which was compressing the powder. More research into optimum processing conditions is required before SLS could be used for the production of intricate channel networks. 


\section{Table of Contents}

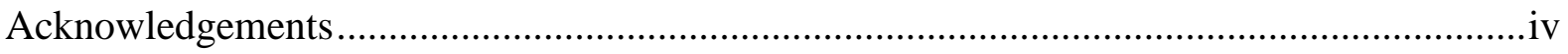

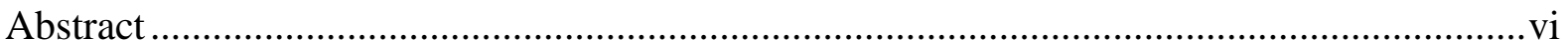

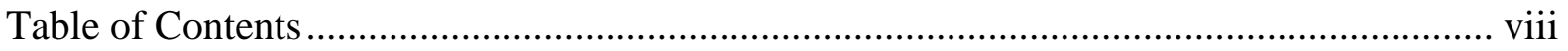

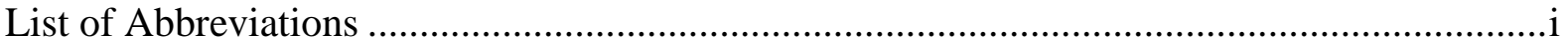

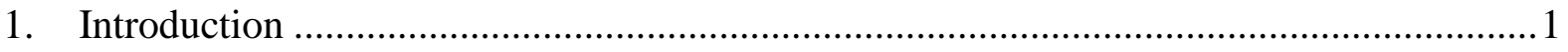

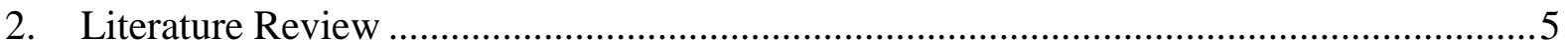

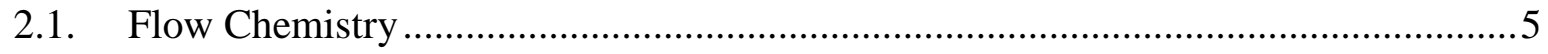

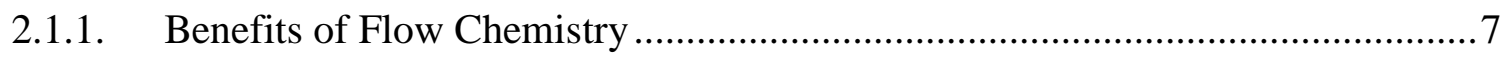

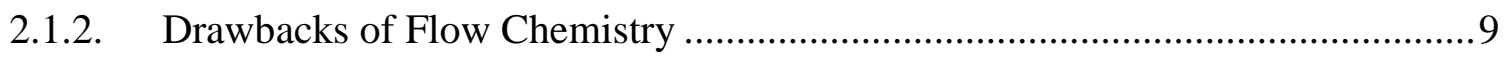

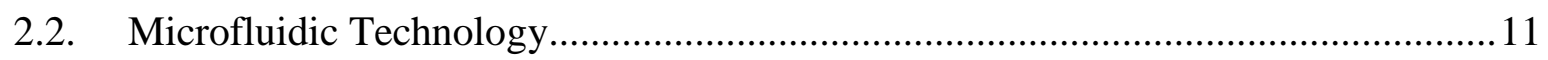

2.3. Microfluidic Devices and Manufacturing Methods ............................................. 14

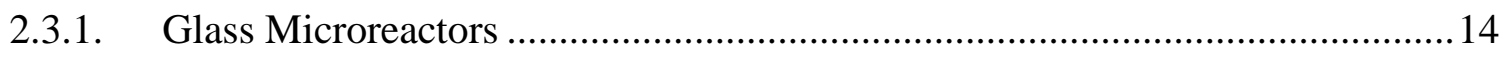

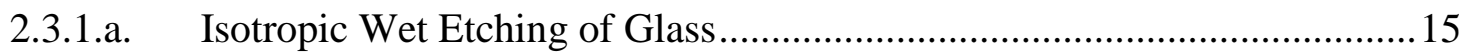

2.3.1.b. Anisotropic Dry Etching of Glass ............................................................. 16

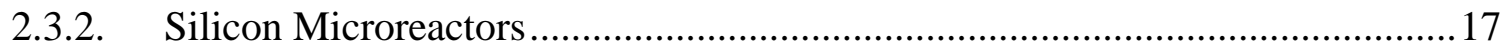

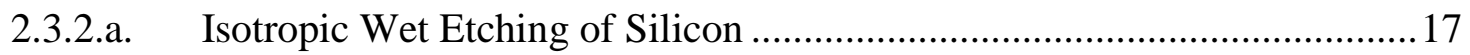

2.3.2.b. Anisotropic Wet Etching of Silicon.................................................... 18

2.3.2.c. Isotropic Dry Etching of Silicon......................................................... 18

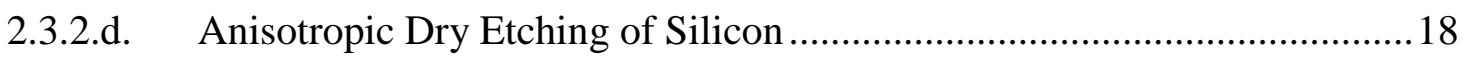

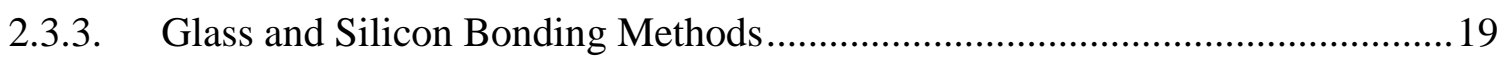

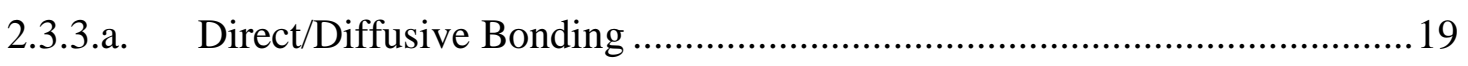

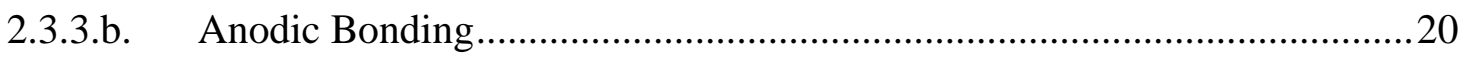

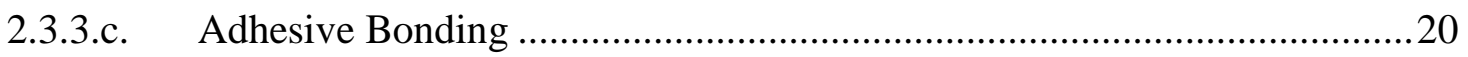

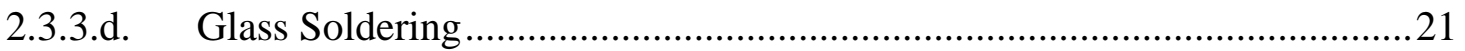

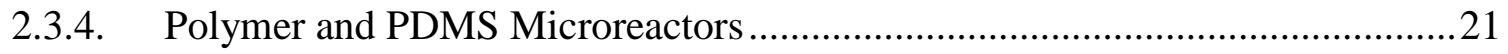

2.3.4.a. $\quad$ Rapid Prototyping and Replica Moulding of PDMS ...............................21 


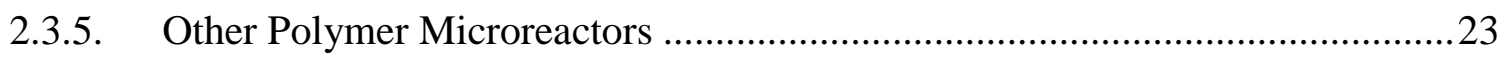

2.3.5.a. Injection Moulding of Polymer Microreactors .........................................2 23

2.3.5.b. Reaction Injection Moulding of Polymer Microreactors............................24

2.3.5.c. Hot Embossing of Polymer Microreactors .................................................2 24

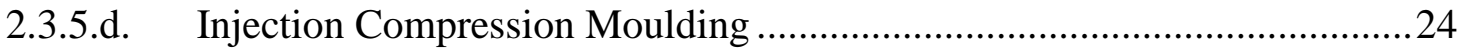

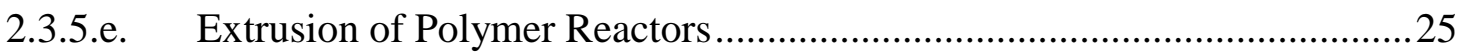

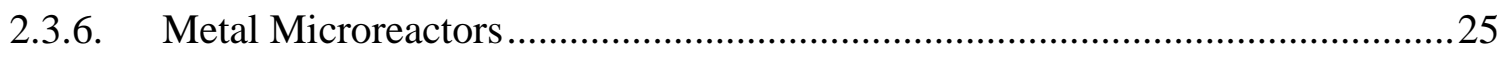

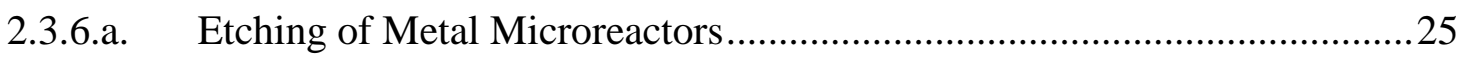

2.3.6.b. Micro-machining of Metal Microreactors ..................................................25

2.3.6.c. Metal Forming Techniques....................................................................... 26

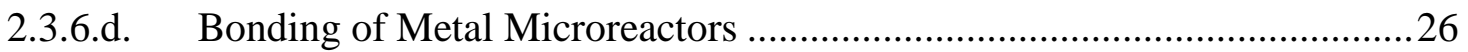

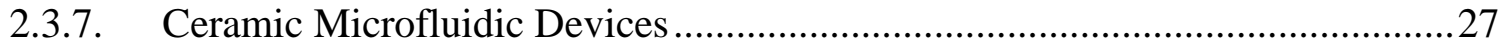

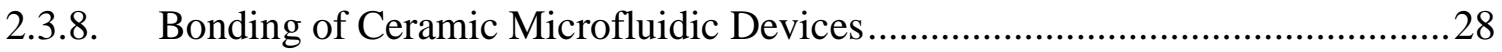

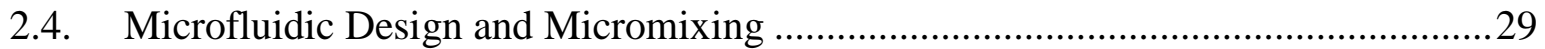

2.4.1. Defining Microfluidic Flow Regimes .........................................................229

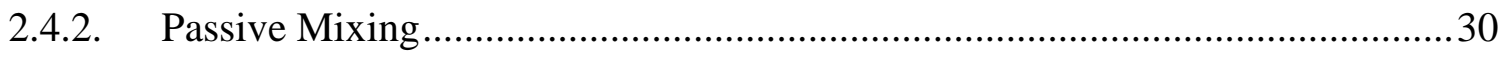

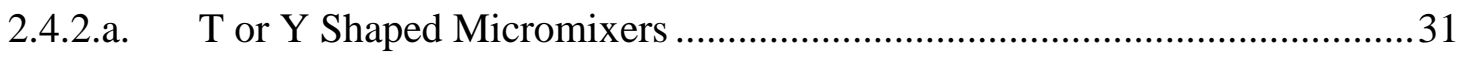

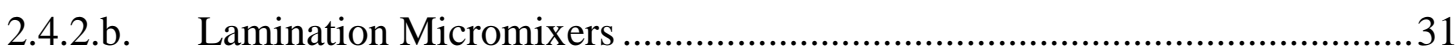

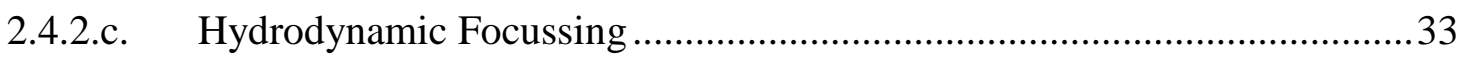

2.4.2.d. Chaotic Advection Micromixers ................................................................. 34

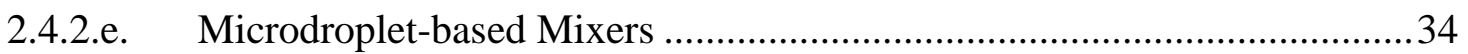

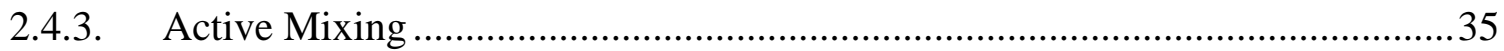

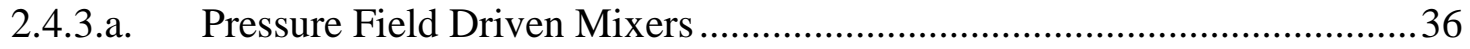

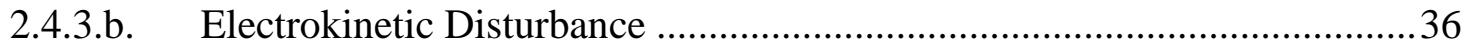

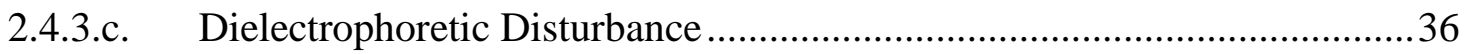

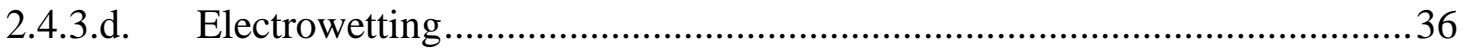

2.4.3.e. Magneto-Hydrodynamic Disturbance ................................................... 36 
2.4.3.f. Ultrasound Disturbance

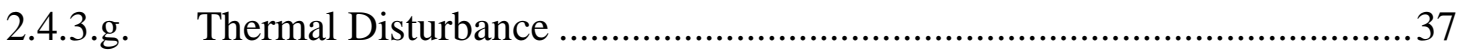

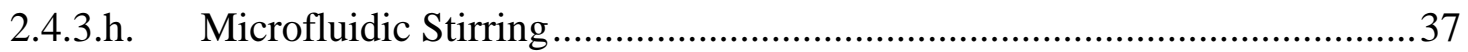

2.5. Integration of Spectroscopic Measurements into Microfluidic Devices ...................39

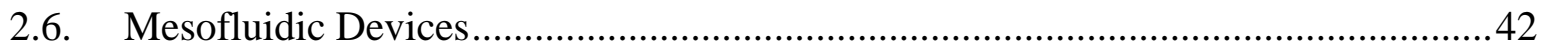

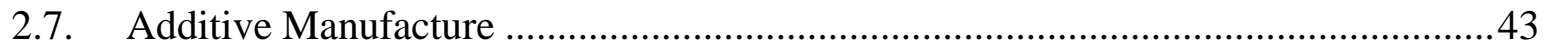

2.8. Additive Manufacture of Fluidic Devices .............................................................. 46

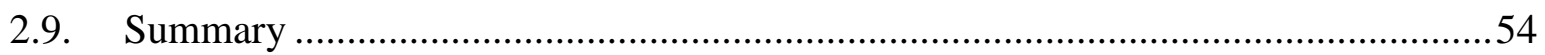

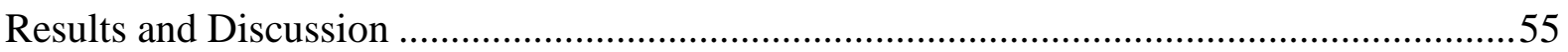

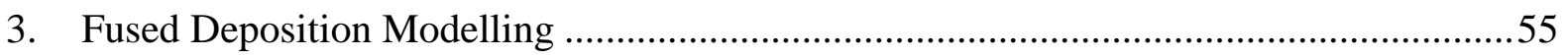

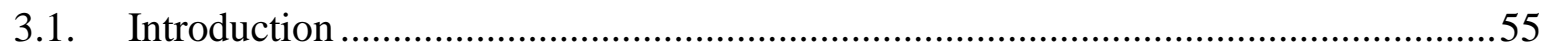

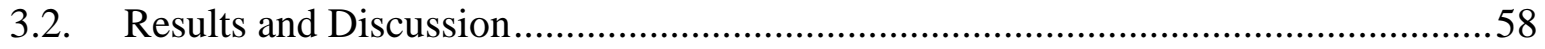

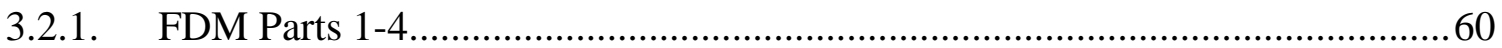

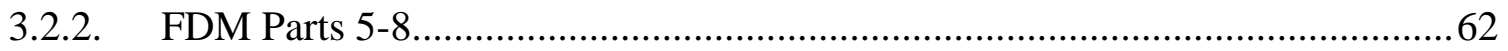

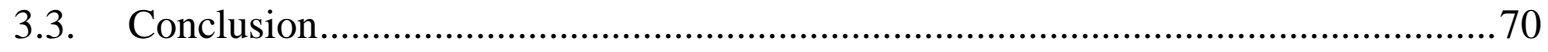

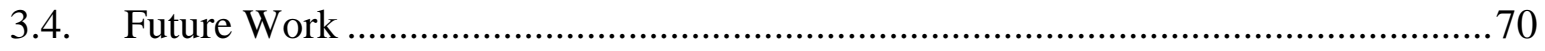

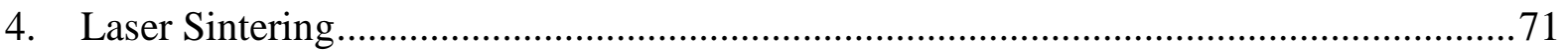

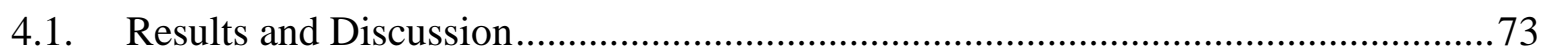

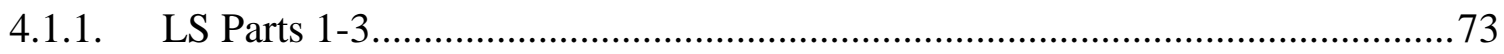

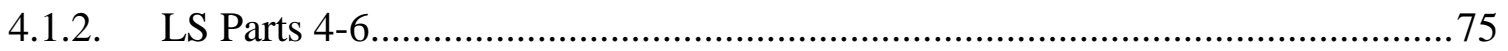

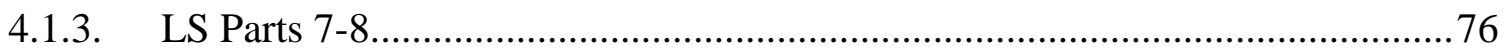

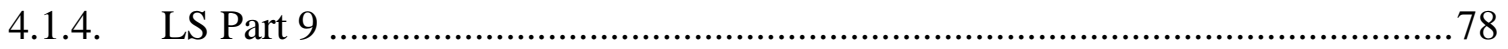

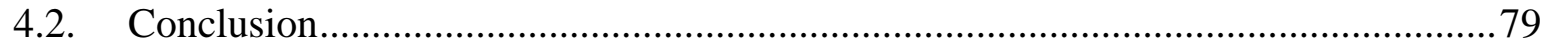

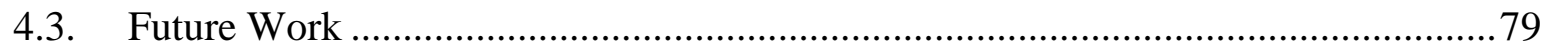

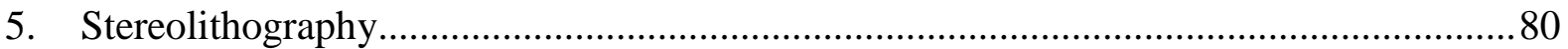

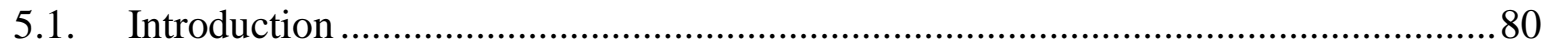

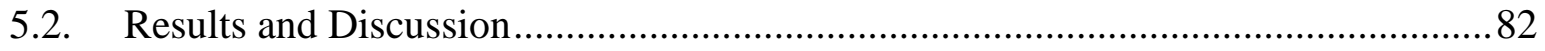




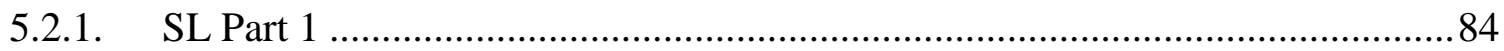

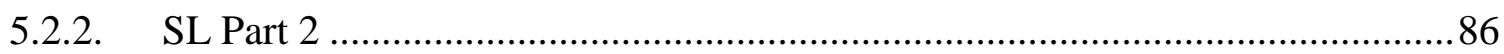

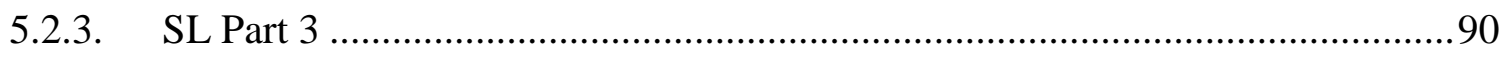

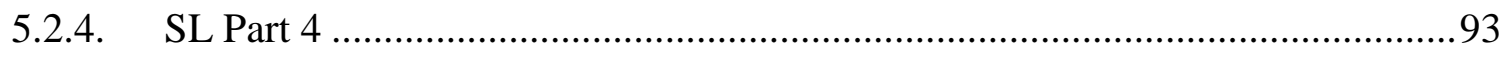

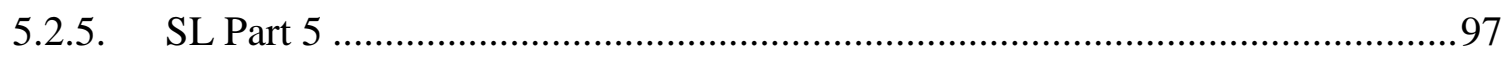

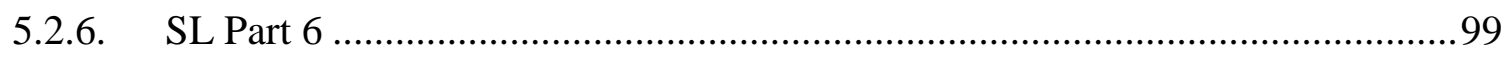

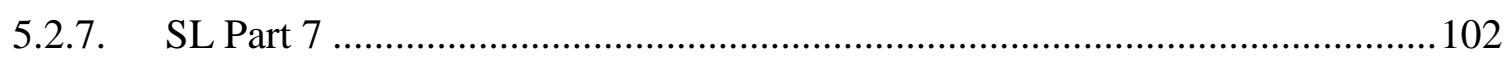

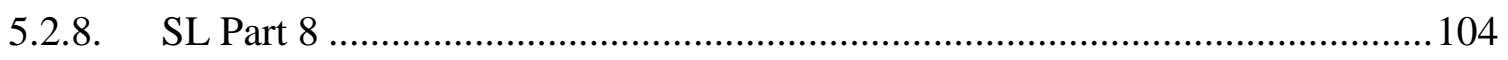

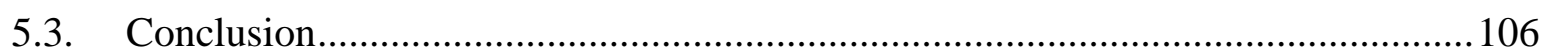

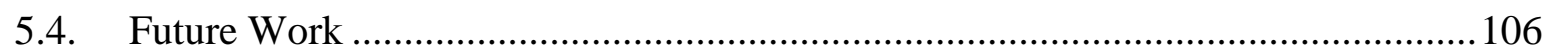

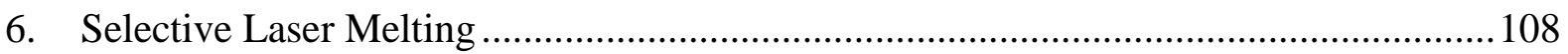

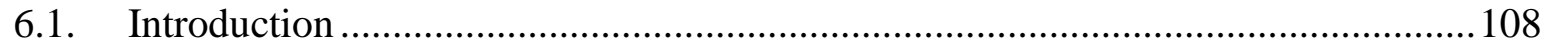

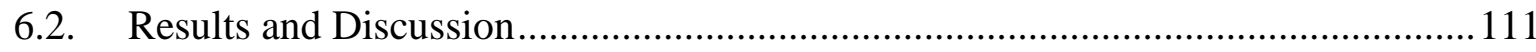

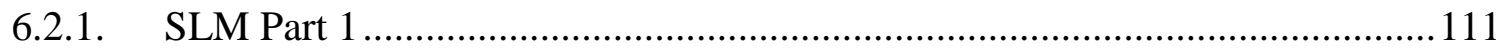

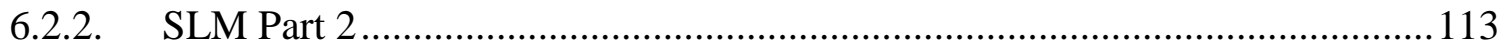

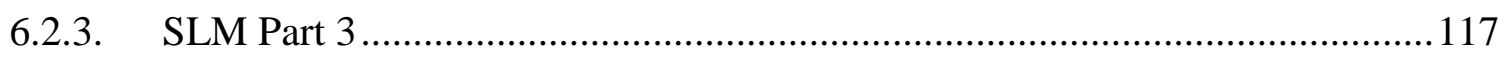

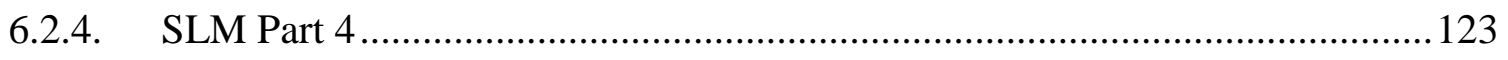

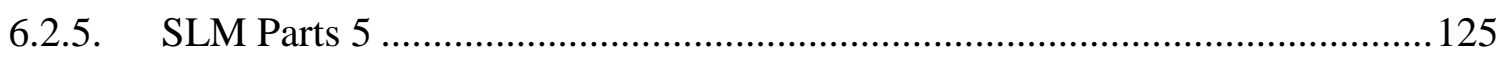

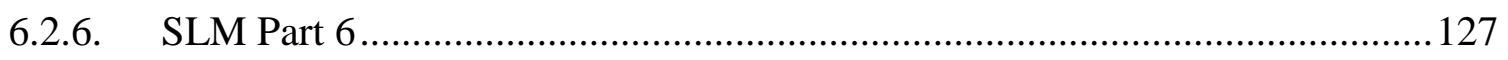

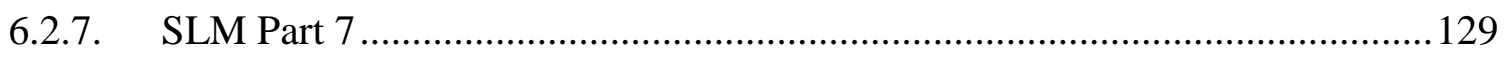

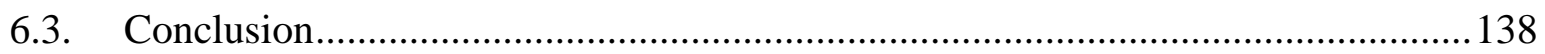

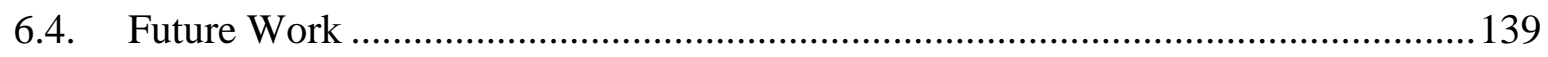

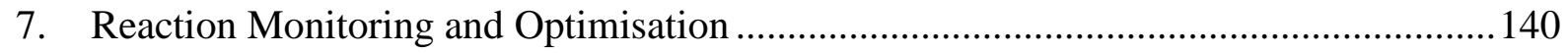

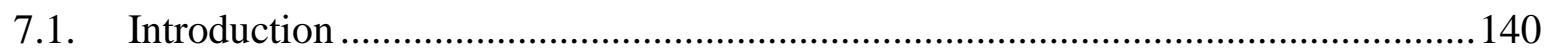

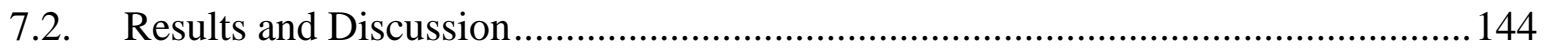

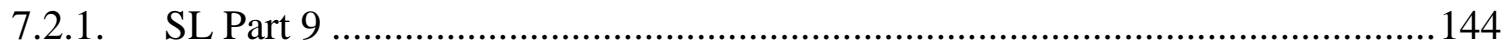

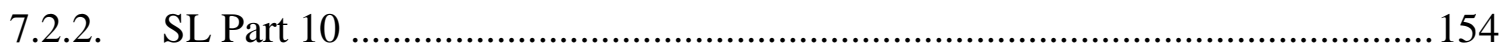


7.2.3. SLM Part 8

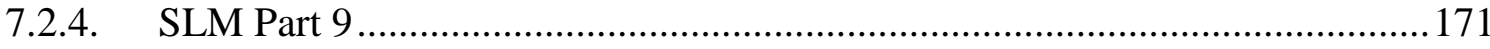

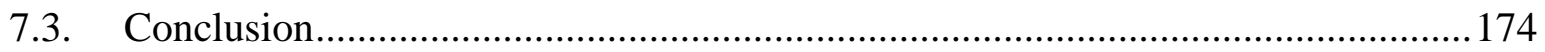

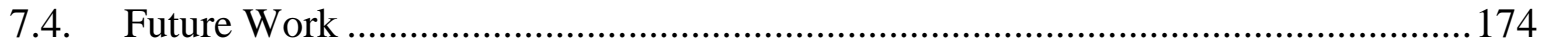

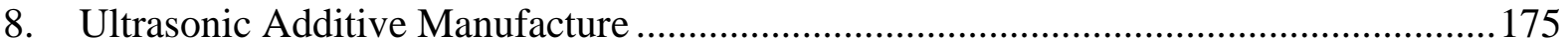

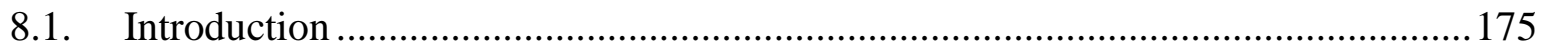

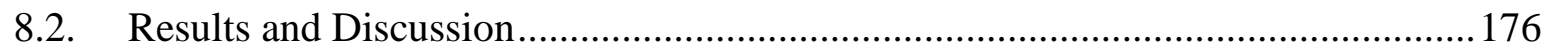

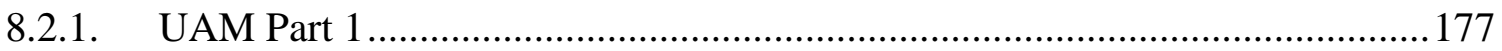

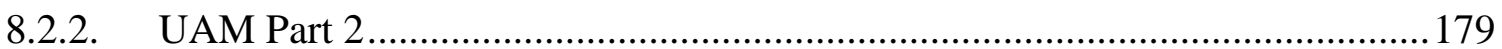

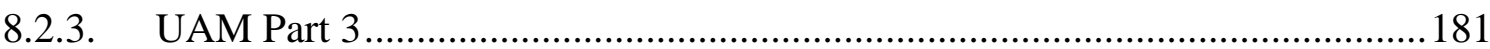

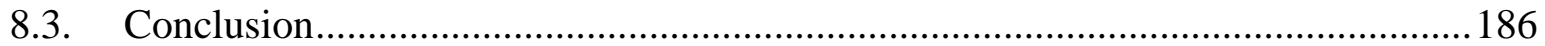

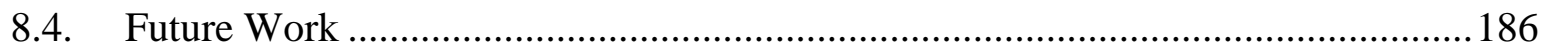

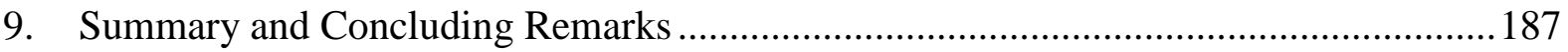

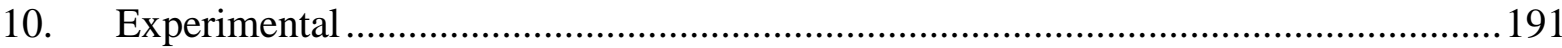

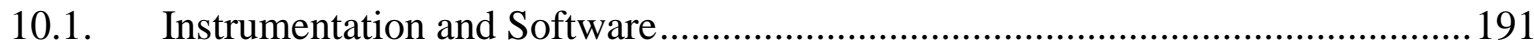

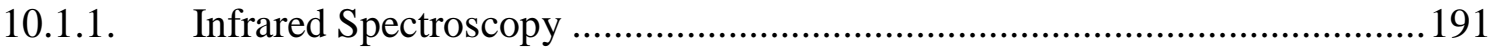

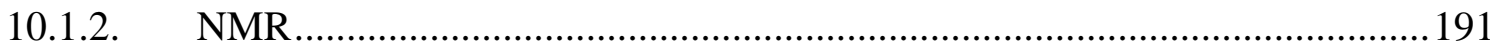

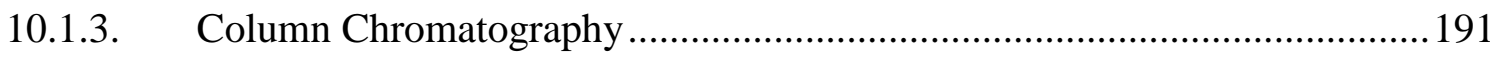

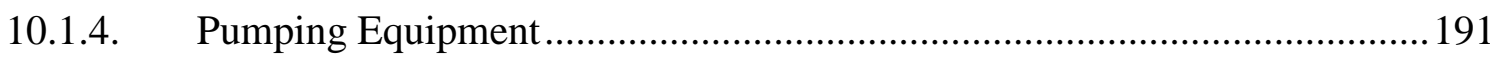

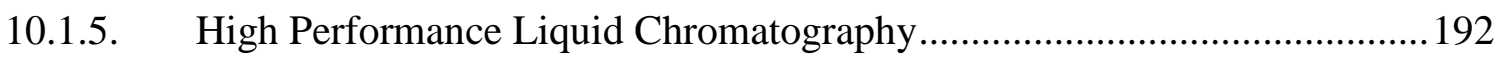

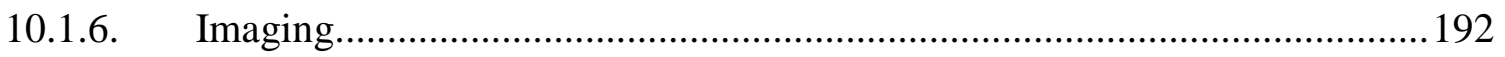

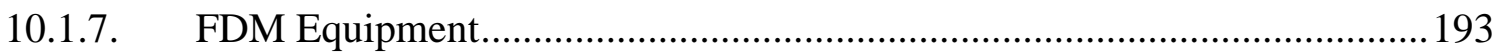

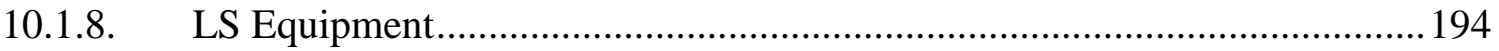

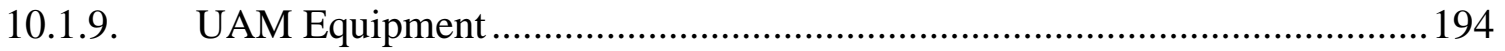

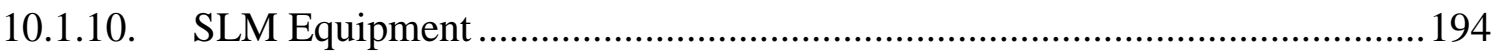

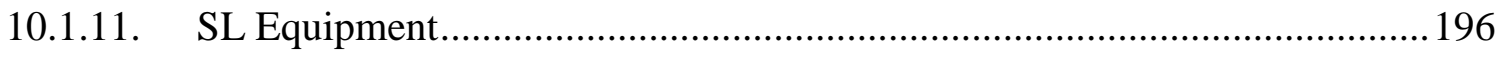

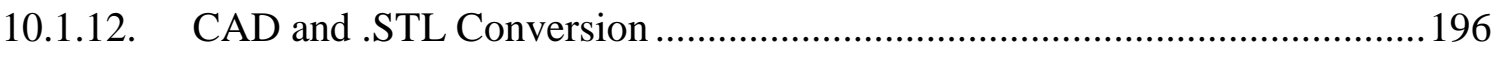




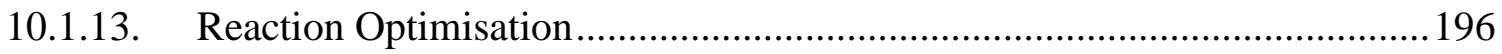

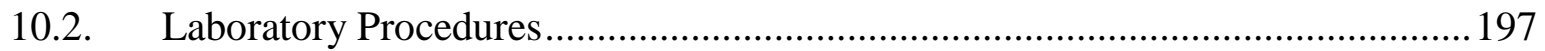

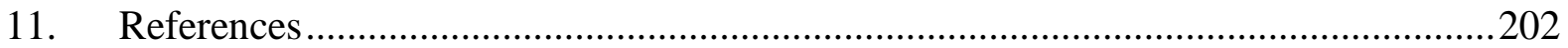

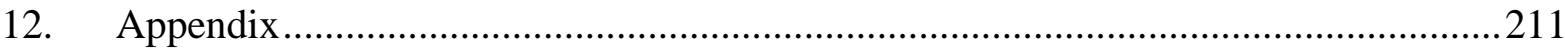

12.1. Design of a Simplex Reaction Optimisation Model ..........................................211

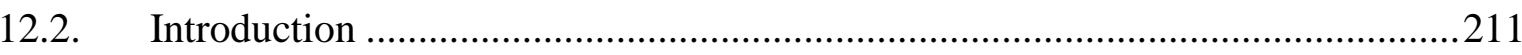

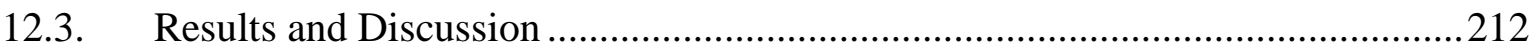

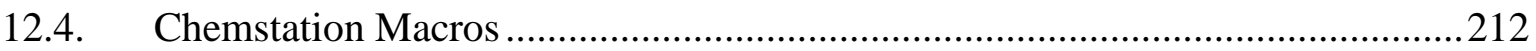

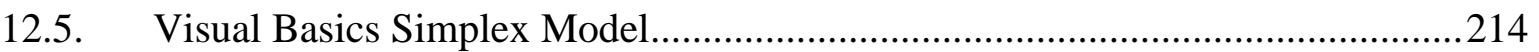

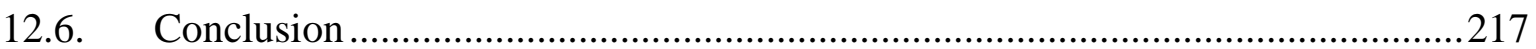

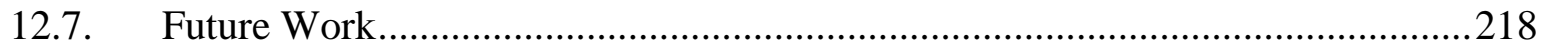

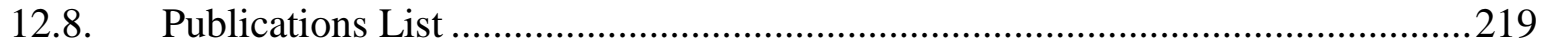

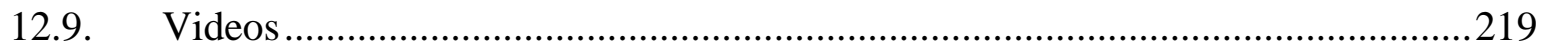

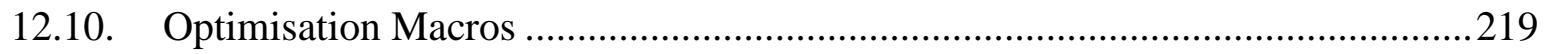




\section{List of Abbreviations}

\section{Abbreviation}

\section{Equipment Used}

${ }^{13} \mathrm{C}$ NMR

Carbon 13 Nuclear Magnetic Resonance Spectroscopy

${ }^{1} \mathrm{H}$ NMR

Proton Nuclear Magnetic Resonance Spectroscopy

FTIR

Fourier Transform Infrared Spectroscopy

IR

Infrared Spectroscopy

HPLC

High Performance Liquid Chromatography

UV-Vis

Ultraviolet-Visible Spectroscopy

SEM

Scanning Electron Microscope

FDM

Fused Deposition Modelling

SL

Stereolithography

$\mathrm{UC}$

Ultrasonic Consolidation

UAM

Ultrasonic Additive Manufacture

SLM

Selective Laser Melting

SLS

Selective Laser Sintering

ATR

Attenuated Total Reflection Spectroscopy

ESI-MS

Electrospray Ionisation Mass Spectrometry

MJM

Multi-Jet Modelling

DAD

Diode Array Detector

Solvents and Reagents

DCM

Dichloromethane

DMF

Dimethylformamide 
THF

TMAH

$\mathrm{BCB}$

TMS

Materials

PTFE

PEEK

PDMS

FEP

PC

PVC

PMMA

PP

\section{Units of Measurement}

$\mathrm{h}$

$\mathrm{min} / \mathrm{m} / \mathrm{mins}$

s

d

$\mathrm{t}$

dt

ddd

$\mathrm{m}$

sept
Tetrahydrofuran

Tetramethyl Ammonium Hydroxide

Benzocyclobutene

Tetramethylsilane

Polytetrafluoroethylene

Polyether Ether Ketone

Polydimethylsiloxane

Fluorinated Ethylene Propylene

Polycarbonate

Polyvinylchloride

Poly(methyl methacrylate)

Polypropylene

Hours

Minutes

Seconds/Singlet

Doublet

Triplet

Doublet Of Triplets

Doublet of Doublets of Doublets

Multiplet or Metres

Septuplet 


\begin{tabular}{|c|c|}
\hline KW & Kilowatts \\
\hline $\mathrm{K}$ & Kelvin \\
\hline$\mu \mathrm{m}$ & Micrometres \\
\hline$£$ & Pounds Sterling \\
\hline $\mathrm{nm}$ & Nanometres \\
\hline $\mathrm{mm}$ & Millimetres \\
\hline mTorr & Millitorr \\
\hline${ }^{\circ} \mathrm{C}$ & Degrees Celsius \\
\hline psi & Pounds Per Square Inch \\
\hline $\mathrm{MPa}$ & Megapascal \\
\hline $\mathrm{kg}$ & Kilogram \\
\hline $\mathrm{ms}$ & Millisecond \\
\hline $\mathrm{mL}$ & Microlitre \\
\hline ppm & Parts Per Million \\
\hline $\mathrm{MHz}$ & Megahertz \\
\hline $\mathrm{Hz}$ & Hertz \\
\hline $\mathrm{mg}$ & Milligram \\
\hline $\mathrm{g}$ & Gram \\
\hline mol & Mole \\
\hline mmol & Millimole \\
\hline KV & Kilovolts \\
\hline$\mu \mathrm{A}$ & Microamps \\
\hline $\mathrm{MHz}$ & Megahertz \\
\hline
\end{tabular}


MW

in

\section{General Terms}

AM

UNF

$t_{R}$

3-D

RF

$\sim$

MEMS

2-D

CAD

$\mu \mathrm{TAS}$

Re

Pe

St

$f$

$\mathrm{u}$

L

D

$\mathrm{P}$

$\mathrm{D}_{\mathrm{h}}$

$\mu$
Megawatts

Inch

Additive Manufacture

Unified Fine Thread

Residence Time

Three dimensional

Radio Frequency

Approximately

Microelectromechanical Systems

Two Dimensional

Computer Aided Design

Micro Total Analysis Systems

Reynolds Number

Péclet Number

Strouhal Number

Frequency

Fluid Velocity

Length

Mass Diffusion Coefficient

Fluid Density

Channel Diameter

Dynamic Viscosity 
Kinematic Viscosity

SAR

Split And Recombine

$\mathrm{Ca}$

Capillary Number

$\gamma$

Interfacial Tension

MHD

Magneto-Hydrodynamic Disturbance

LED

Light Emitting Diode

STL

Surface Tessellation Language

PEM

Proton Exchange Membrane

ATP

Adenosine Triphosphate

HD

High Definition

TLC

Thin Layer Chromatography

RIE

Reactive Ion Etching

RT

Room Temperature

MHD

Magneto-Hydrodynamic Disturbance

VB

Visual Basics

Aq.

Aqueous 


\section{Introduction}

The last few decades have seen the fields of flow chemistry and microfluidics become well established methods of continuous flow processing. The applications for these technologies range from the manufacture of small molecules and pharmaceuticals, to biological screening, and have been demonstrated to have some significant advantages over traditional batch processing methods. These advantages include efficient mixing of reagents, superior handling of hazardous materials, and the ability to automate and optimise the flow regime. Unfortunately, issues associated with this type of processing, ranging from equipment/training cost, handling of precipitates, and lack of user familiarity with this type of work regime, often lead to these methods being overlooked for more familiar batch manufacturing. One particular difficulty associated with flow chemistry is the manufacture of microfluidic devices which can often be costly and time consuming to produce, as well as being limited in the design of the device and the material from which it can be manufactured.

It was therefore proposed to use a range of novel additive manufacturing (AM) or "3D Printing" processes to manufacture multi-functional flow devices for flow chemistry applications. AM has a significant number of advantages over more traditional subtractive manufacturing processes, most notably the substantially increased design freedom and the ability to prototype and produce parts often within the same day. This would hopefully allow the manufacture of flow devices with increased functionality, incorporating more complex and intricate mixing pathways, whilst integrating a range of analytical instrumentation into the device that could increase the functionality of the part. The data generated from this instrumentation could be used to provide instantaneous feedback about the flow process, which could be integrated with sophisticated reaction optimisation and equipment control software to create a highly efficient and automated flow system. The design freedom afforded by AM would allow these flow devices to be integrated with existing laboratory instrumentation to prevent the need for the purchase of expensive flow and analysis equipment.

At the time of commencing the project there had been no literature precedent for the use of AM for the manufacture of microfluidic type devices. AM offers a significant number of different manufacturing processes, each of which come with their own considerations in terms of material selection, design freedom, cost, and production time. Throughout this project, five AM processes were used and reviewed including stereolithography (SL), 
selective laser melting (SLM), fused deposition modelling (FDM), ultrasonic additive manufacture (UAM) and selective laser sintering (SLS). The initial aims of this project would therefore be targeted at gaining a fundamental understanding of the advantages and disadvantages of manufacturing flow devices using each of these different processes. This would allow the development of a dataset of parameters such as typical build resolution, processable and available materials, as well as mechanical properties of the parts produced by each process. This research began by designing and testing parts using FDM, the most readily available AM process, with the hope of being able to manufacture inexpensive and disposable fluidic devices. However, more advanced printing processes within the group such as SL, LS and SLM were also available within the research group and therefore the functionality of each of these three processes was also tested upon completion of the FDM work. Having successfully tested each of these processes and developed a wider understanding of the applicability of each to this application, it was possible to develop more complex parts whilst targeting the designs produced from each process to more specific applications.

The next stage of the research would focus on the manufacture of parts which would mimic existing commercially available flow devices. This would initially focus on devices with simplistic sub-millimetre channel designs, with channel networks that ranged in volume from $1 \mathrm{~mL}$ to $20 \mathrm{~mL}$, replicating the typical milli-scale reactors available upon purchase of a commercial flow system. The research would then look to move towards the manufacture of parts with complex and intricate flow pathways, and microlitre scale reactor volumes that are typical of the more expensive microfluidic devices which are commercially available. Finally the research will look to focus on the manufacture of devices which would be difficult or impossible to manufacture via conventional subtractive processes, incorporating highly complex channel designs and reactor geometries. This research largely focussed on the use of the SL and SLM processes. These two processes were selected to provide a set of manufacturing techniques that could produce parts which were both geometrically precise as well as being thermally, chemically and physically stable across a wide range of different reaction conditions.

Following this, the research would focus on the design of more multifunctional and analytical flow devices, incorporating embedded sensors and detectors, temperature controlled reaction pathways, and designs which allowed the devices to be integrated with existing flow and analytical chemistry apparatus. These reactors would hopefully allow the user to generate instantaneous data about the progression of the reaction, as well as affording the chemist 
greater control over the specific reaction environment. Again this work would focus on the use of the SL and SLM processes, however would also feature the use of UAM. The UAM process was selected for this application with the intention of utilising the processes' ability to embed functionality into the part. The final stage of the research will investigate the possibility of using the data generated from these multi-functional flow devices, as part of a fully automated flow system. This would allow the outputted reaction data would be used to automate the control of parameters such as the flow rate, reaction temperature and pressure, as well as the reagent concentrations. This type of system could be incorporated with a piece of optimisation software, allowing the system to not only identify the optimal flow conditions for each novel reaction undertaken, but automatically control the selection of each new set of reaction conditions.

This thesis presents the methodology used to manufacture such devices, as well as familiarising the reader with the existing manufacturing process currently used to the produce microfluidic devices typically used in flow applications. A short literature review follows this introduction, designed to make clear the origins of the project idea. The literature reported gives a brief introduction into current flow chemistry applications as well as the fluidic reactors used during these processes. It also gives details of the common manufacturing processes used to produce these reactors, as well as a critique of each technique. The section also looks at the range of miniaturised components which have been incorporated into these devices to increase functionality of the part, such as the microfluidic static and dynamic mixers used to improve microfluidic mixing, as well as typical analytical devices which have been used to identify and quantify chemical reagents both inline and online in flow. Finally, the review will analyse the AM techniques proposed to be used during this research, and the specific advantages and disadvantages of using each process. This will also include a summary of the limited number of flow devices which have been manufactured via AM, and discuss the potential impact they may have on future flow applications.

Chapter 3 discusses the use of FDM to produce a number of functional flow devices. FDM was mainly used as an inexpensive method of prototyping parts for more expensive processes, however it was also proposed as a method of embedding discrete components into flow devices.

Chapter 4 covers the use of SLS as a method of method of manufacturing simplistic channel networks. This is followed by chapter 5 which introduces the use of SL as a method of 
manufacturing milli-scale flow devices. This section reports the manufacture of eight flow devices, which were tested using a number of organic and inorganic reactions. A discussion about the suitability of this AM process for these applications is also included.

Chapter 6 covers the use of SLM as a method of manufacturing milli-scale flow devices. Seven flow devices, which were manufactured via SLM, are introduced in this section detailing the specific design and engineering issues which must be overcome to utilise this AM technique. The section also attempts to quantify the precision of this manufacturing process by visualising the parts via scanning electron microscopy, optical microscopy and micro-computed tomography.

Chapter 7 discusses the use of both SL and SLM manufacturing techniques, to incorporate analytical functionality into our devices. The four parts manufactured were used as part of an automated reaction optimisation platform, which allowed us to purify, identify and quantify our reaction medium. These devices were tested by optimising an organic functional group interconversion, and analysing the data via inline UV spectroscopy as well as online HPLC.

Chapter 8 discusses the design and creation of a reaction optimisation platform. This platform includes the design of a piece of software written in Microsoft Office VB which is based around the use of the simplex method of reaction optimisation. As well as this the section discusses the design and development of a range of bespoke macros, which were used to manipulate the Chemstation software used to run an Agilent LC system. These macros allowed the automated control of the pumps, injection valves, heating blocks, chromatographic separation and UV DAD incorporated into a standard LC setup. The combination of these two pieces of software allowed us to develop a completely automated user friendly flow platform.

Finally, chapter 9 discusses the use of UAM to produce a number of functional flow devices. UAM was investigated as a method of embedding thermocouples into an AM part, which could be used as an analysis tool in the optimisation platform discussed in chapter 7 . 


\section{2. $\quad$ Literature Review}

\subsection{Flow Chemistry}

The field of chemistry has for many years relied on batch and semi-batch processes based around the use of glassware to manufacture, analyse and purify chemical compounds. ${ }^{1}$ In recent decades, technological advances have opened up a field of chemistry known as flow chemistry, based around the continuous flow of reagents through chemical reactors. ${ }^{2}$ These reactors typically come in the form of micron-sized channels patterned into the surface of a glass or polymer chip (often referred to as "Lab-on-a-Chip"), ${ }^{3}$ or more simply as lengths of tubing typically made from stainless steel, or inert polymers such as polytetrafluoroethylene (PTFE), or polyetheretherketone (PEEK). ${ }^{4}$ Reactors can be integrated with other flow accessories via the use of fluidic connectors; compression fittings or ferrules (usually PEEK) slide over the end of this tubing and form a tight seal when mated with a second fluidic component. These ferrules are held in place by the pressure generated from tightening a nut into the flow components cavity. Typically these nuts are supplied as either $1 / 4-28$ or 10-32 UNF threaded PEEK fittings. ${ }^{5}$ Reactors are connected to reservoirs of reagent solutions, which are moved along the reaction channels by external pumps. A range of pumping systems are available, such as hydrodynamic, electrokinetic, or capillary flow action pumps. ${ }^{6}$ Flow throughout the system is generally laminar (scale dependent), with mixing of reagents being achieved by diffusion, however turbulent mixing is often incorporated into many reactor pathways, with the aim of improving mixing, and consequently improving reaction efficiency. ${ }^{7}$ It is also possible to heat and cool reactions efficiently via the use of integrated heating and cooling blocks. ${ }^{8}$ The reagents are mixed prior to or upon entering the reactor, usually by a simplistic $\mathrm{T}$ or $\mathrm{Y}$ shaped mixer, however more complex mixers can be implemented. ${ }^{9}$ The time that the reagents spend in the reactor is therefore a function of the flow rate of the pump(s) and the volume of the reactor, and is termed the "residence time" (tR). Reaction products can be monitored inline (analysis without breaking the reagent stream) using analytical methods such as IR (infrared spectroscopy) or UV-Vis (UltravioletVisible) spectroscopy whereby the probe/sensor is placed directly into the flow stream, ${ }^{10}$ or online (by taking an aliquot of the reaction product out of the flow stream) and analysing it via techniques such as high performance liquid chromatography (HPLC) or mass spectrometry. ${ }^{11}$ 
Flow chemistry has emerged as a useful technology for research and development chemists within the pharmaceutical sector. The potential benefits of flow chemistry are numerous and well documented, however flow technologies are yet to attain widespread use amongst research chemists. The reluctance of pharmaceutical companies to move away from traditional chemistry practices is understandable, as flow technologies still suffer from a number of fundamental issues, such as their inability to effectively handle solid compounds and precipitates. On top of this, the cost of moving away from established batch processes would be substantial. It is likely that a complete transition would only be made if this financial deficit was offset by a substantial increase in productivity. Despite this, these reaction platforms are gradually integrating themselves into pilot plants, and even large scale production lines of pharmaceutical companies across the globe, with major pharmaceutical companies now investing heavily in continuous flow production of target compounds. ${ }^{12}$ It is estimated that as many as $50 \%$ of reactions in the fine chemical and pharmaceutical industry could benefit from continuous flow processing. ${ }^{13}$ Hence, continuous flow processing is now widely used within the pharmaceutical industry for both large scale productions of chemical products, as well as part of research and development programmes. Consequently a selection of commercially available flow platforms are now available to meet the wide ranging demands of the modern laboratory (Figure 1). ${ }^{14}$ These platforms can be integrated with liquid handling systems, analytical flow devices and complex optimisation software to create a fully automated flow chemistry platform. However, many research chemists still opt to build their own systems, using low cost PTFE or stainless steel tubing, a simplistic T-piece or Y-piece and syringe pumps, for a fraction of the cost. 


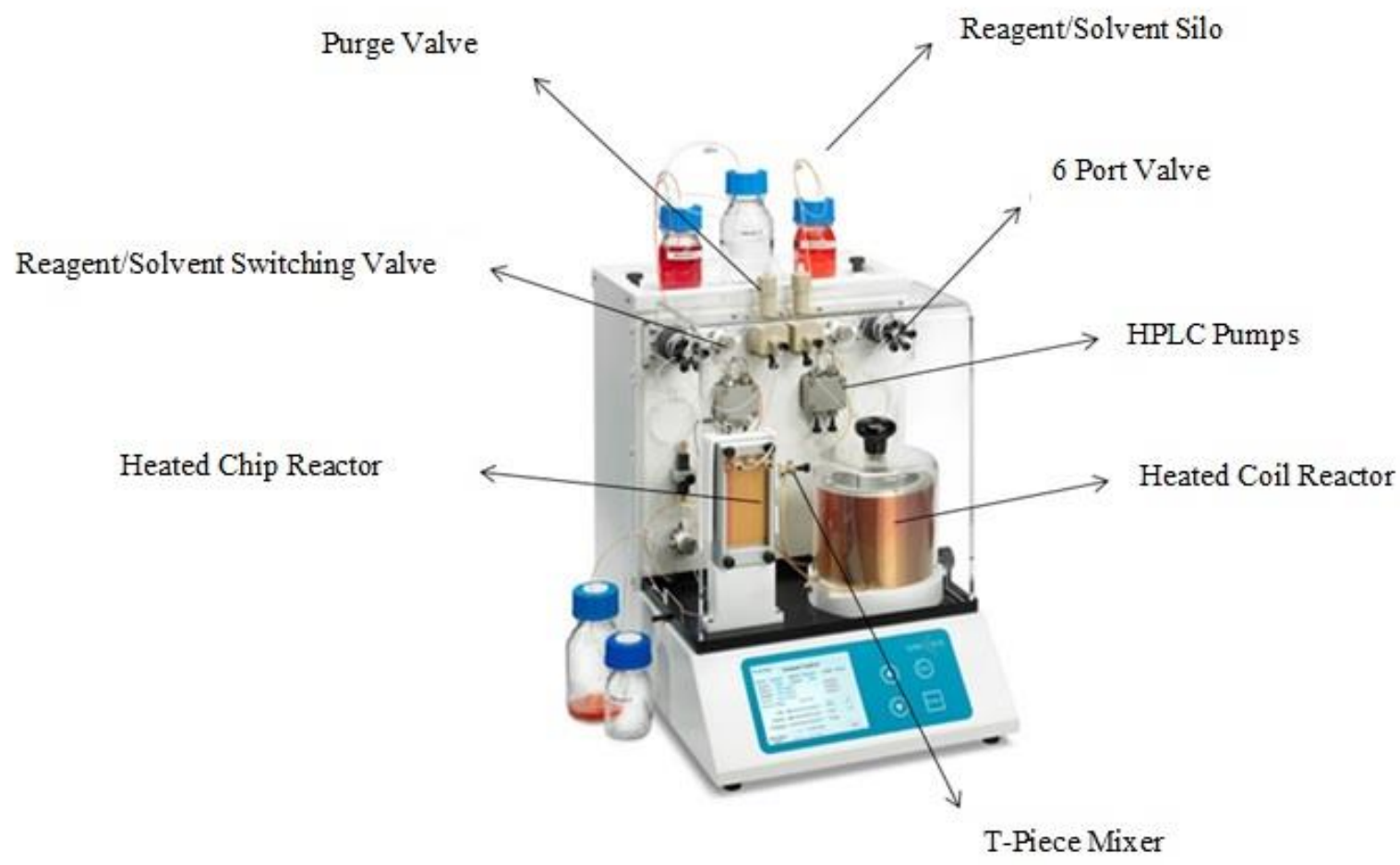

Figure 1: The Uniqsis FlowSyn is an example of a commercially available flow chemistry apparatus. ${ }^{15}$

\subsubsection{Benefits of Flow Chemistry}

The miniaturisation of the reaction pathways utilised in flow chemistry have a number of distinct advantages over more conventional batch laboratory techniques:

Selectivity: many chemical processes generate more than one reaction product from the same reagent pool depending on external environmental conditions. In microfluidics, the precise control over many of these conditions, such as pressure and temperature, affords the chemist some control over the selection of one product over another. ${ }^{16}$

Enhanced mass transfer: enhanced heat and mass transfer throughout the reaction medium leads to more frequent and uniform molecular interactions during a flow process. Typically, microreactors demonstrate a much larger surface area to volume ratio than conventional batch reactors such as round bottom flasks or test tubes, minimising diffusion distances within the reaction. Microreactors with surface area to volume ratios of $\sim 10,000$ to $50,000 \mathrm{~m}^{2} . \mathrm{m}^{-3}$ are not uncommon, ${ }^{4}$ in comparison to typical batch reactors with surface area to volume ratios of $\sim 4 \mathrm{~m}^{2} \cdot \mathrm{m}^{-3}$. These values increase exponentially as the internal channel dimensions of a 
microreactor decrease. The distribution of reagents throughout the microreactor is also very regular when compared with conventional glassware reactors, again leading to more regular molecular interactions and uniform distribution of molecular residence times.

Enhanced heat transfer: similarly the distribution of heat throughout the microreaction system is much more uniform than when using batch reactors, providing the chemist with greater control over the reaction thermodynamics. It is again believed that this heat transfer is a result of the large surface area to volume ratio associated with microreactor systems. ${ }^{4}$ Typically microreactors demonstrate heat transfer coefficients of $\sim 10$ to $25 \mathrm{~kW} \cdot \mathrm{m}^{-2} \cdot \mathrm{K}^{-1}$ which are significantly higher than traditional batch heat exchangers. ${ }^{4}$ This effect allows chemists to heat and cool reactions in a highly efficient manner, allowing precise control over reaction thermodynamics.

Regular flow profiles: micro-sized reactors demonstrate extremely regular flow profiles throughout the reaction medium. These flow profiles are mostly laminar and highly symmetric. When using automated reactor systems, such precise control over reaction conditions can often lead to substantial time reductions in developing optimised reaction conditions. $^{17}$

Hazardous reaction products: microreactor technology also allows for the safe handling of many hazardous reaction products or intermediates that are produced during a reaction, due to the small quantities being generated at a given time, and the closed environment being used. For example, the production of tetrazole rings can be carried out relatively safely by utilising flow chemistry, however this reaction can often be deemed too dangerous to carry out in batch due to the potentially explosive azide starting material. In flow however, as only small quantities of the reagents are exposed to the reaction environment at any given time, the risks associated with the hazardous material are minimized. ${ }^{18}$

Exothermic reactions: due to the efficient heat transfer associated with microfluidic flow, microreactors are ideal tools for carrying out highly exothermic reactions. Exothermic reaction products can be produced on such small scales, that any localised exotherms are dispersed safely across the reaction profile. Therefore, the development of hot spots, or the accumulation of localised areas of heat are supressed. The outcome for a number of reactions is an increase in reaction yield, purity and selectivity. ${ }^{4}$ 
Small reagent volumes: microfluidic devices often consume fewer reagents than bulk systems to gather the same analytical data. This leads to a considerable reduction in processing cost, particularly when dealing with costly reagents or reactions where only small volumes are available for testing and analysis. ${ }^{16}$

Green chemistry: a number of factors such as reduced thermal output, increased reaction selectivity, reduced reaction volumes, and reduced solvent and reagent usage etc., can lead to substantial environmental benefits when using flow chemistry instead of batch processing. ${ }^{19}$

Reaction optimisation and automation: with the advent of more precise pumping systems and the development of highly robust analytical procedures, the fields of reaction optimisation, experimental design, and reaction automation have become intrinsically linked with flow chemistry applications. Within the last decade these processes have gained wide spread interest in research environments, both industrially and academically, with the aim of substantially reducing product development life cycles. ${ }^{20}$

\subsubsection{Drawbacks of Flow Chemistry}

There are however, still areas within the field of flow chemistry, in which substantial development and research still needs to be undertaken;

Solid precipitates: flow reactors are largely unable to deal with solid reactants and precipitating products, which cause increasing pressure and blockages within the flow system. Such blockages will inevitably lead to a breakdown in continuous processing and cause substantial setbacks in time and expense. Reactions are therefore generally designed so that the starting material, product and any by-products are all soluble in the same solvent system. Solid supported catalyst beds, and reaction purification units can be incorporated into flow using packed bed columns within the flow path. ${ }^{21}$

Expense: to establish flow chemistry as a major contributor in modern pharmaceutical and research chemistry would require significant investment in both new equipment and operator training. It would also require huge research and development output, in order to develop effective flow processes which were capable of mimicking or improving upon established batch synthetic processes. ${ }^{22}$ 
Multi-step reactions: chemical synthesis of fine chemicals and pharmaceuticals are often complex, involving multiple reaction and separation steps, each with alternate pathways and technical approaches. Reaction complexity in flow increases substantially as the potential number of reaction steps increases, making the production of many pharmaceutical targets incredibly complex. Continuous processing and batch processing can be used in tandem to overcome these issues facilitating an optimised reaction set up. 


\subsection{Microfluidic Technology}

Microfluidic systems are those which control and manipulate fluids that are constrained within environments that have internal dimensions of a micrometre scale. A generally accepted definition of a microfluidic device, would be when the characteristic channel dimensions are below $500 \mu \mathrm{m} .{ }^{16}$ A more robust definition may be a device whereby fluid flow operates below Reynolds $(R e)$ and thermal Péclet numbers $(P e)$ below 250 and 100 respectively. Although the first reports of microfluidic systems date back to the 1940 's, ${ }^{23}$ the origins of mainstream microfluidics began in the $1990 \mathrm{~s}^{24}$ This led to a considerable drive in the field of microfluidic technology, with notable contributions from GlaxoSmithKline, ${ }^{25}$ Massachusetts Institute of Technology, ${ }^{26}$ and University College London. ${ }^{27}$ Consequently, over recent years, microreaction technology has become one of the most rapidly developing research fields in synthetic and process chemistry. These devices have evolved from relatively simplistic channel networks used for basic chemical processes, right through to complex designs for use in multi-step syntheses. ${ }^{20}$ This development has been driven by the need to develop efficient mixing technologies, as well as the ability to cope with highly exothermic and potentially explosive processes. ${ }^{28}$ These microfluidic devices have been shown to enhance product selectivity, reaction yields, as well as making considerable steps in the field of reaction optimisation and automation. ${ }^{29}$

Microfluidic reactors which flow reactants across 2-dimensional channels networks are typically known as chip reactors or "lab on a chip". The applicability of different microreactors are defined by their size and internal channel designs, the material from which they are manufactured, and also the type of reaction medium/solvent system required. ${ }^{30}$ These chips are generally micro-scale in dimension, and can often demonstrate excellent mixing properties. ${ }^{31}$ The small volume capacities of microfluidic devices considerably reduce the quantities of material required to optimise reaction conditions, however does bring about difficulties associated with larger scale production of material. To obtain synthetically useful amounts of material, the reactors are simply allowed to run for a longer period of time (scaleout). ${ }^{16}$ Alternatively, several reactors are placed in parallel (numbering up), assuring comparable conditions for each module in the batch. ${ }^{30}$ Typically, flow throughout these devices is laminar, with Reynolds numbers being in the range of 1 to 250 depending on channel dimensions and flow rates. ${ }^{4}$ Although chip reactors can be used independently or in conjunction with other chip reactors, they are often employed as pre-mixers to larger milliscale "coil" reactors. ${ }^{32}$ Microfluidic reactors are often characterised as either active or 
passive, in reference to the micromixers which they usually contain. Active mixing requires an external input such as vibrational or electrical forces to enhance mixing efficiency, whereas passive mixers rely on molecular diffusion and chaotic advection. ${ }^{33}$

For synthetic chemistry, lab on a chip devices are generally manufactured from glass, silicon, metals such as stainless steel, or polydimethylsiloxane (PDMS) (Figure 2). ${ }^{30}$ For many years PDMS was seen as the material of choice, because of the ease with which it can be cast on to a micro-scale mould and then bonded to glass. The elastomeric nature of the material has been exploited to integrate fluidic valves and pumps on-chip, and has simplified the production of multi-layer devices because the soft layers readily conform to each other. This allows the production of relatively inexpensive and dimensionally accurate microfluidic devices. However, PDMS does have limitations as a material choice due to the limited range of chemical and thermal conditions in which it is stable. ${ }^{34}$ Glass is a highly favourable alternative to PDMS as it is chemically inert to almost all reagents and solvents, is stable across a wide range of reaction temperatures and pressures, and is optically transparent. Both glass and PDMS reactors allow clear visual inspection of reactions unlike other types of reactor. ${ }^{35}$ Silicon has also found widespread use in the construction of these devices. When oxidised, silicon behaves with very similar physical properties to glass and is chemically inert to most organic solvents. It also has exceptional thermal conductivity and is therefore ideal for use in reactions where the control of heat or heat transfer is paramount. Silicon reactors are therefore suited to exothermic reactions or reactions that need to be kept at low temperatures. ${ }^{30}$ Typically, chip reactors which are manufactured using chemically etched glass or silicon plates, are expensive to manufacture due to the higher material, energy, and capital equipment costs. Commercially available glass chip reactors cost $£ 1000$ s, with the price increasing dramatically with the increasing complexity of the part. They are also often limited to 2-D flow paths, and are often only suitable for synthesising large quantities of material in conjunction with other chip reactors. All of these considerations often make the use of microfluidic devices highly expensive to set up, maintain, and run.

Microreactors manufactured from metals such as stainless steel and Hastelloy® are also common, being thermally stable, conductive, and suitably inert to a range of reaction mediums. Other materials such as noble metals (e.g. platinum and palladium), as well as alloys of materials such as copper, titanium and nickel have also been used for microfluidic device manufacture. ${ }^{36}$ The dimensions of these reactors are generally greater than those seen in glass and silicon microfluidic devices, making them of particular interest to academic 
research laboratories, ${ }^{30}$ as well as gaining use for high temperature and high pressure reactions.

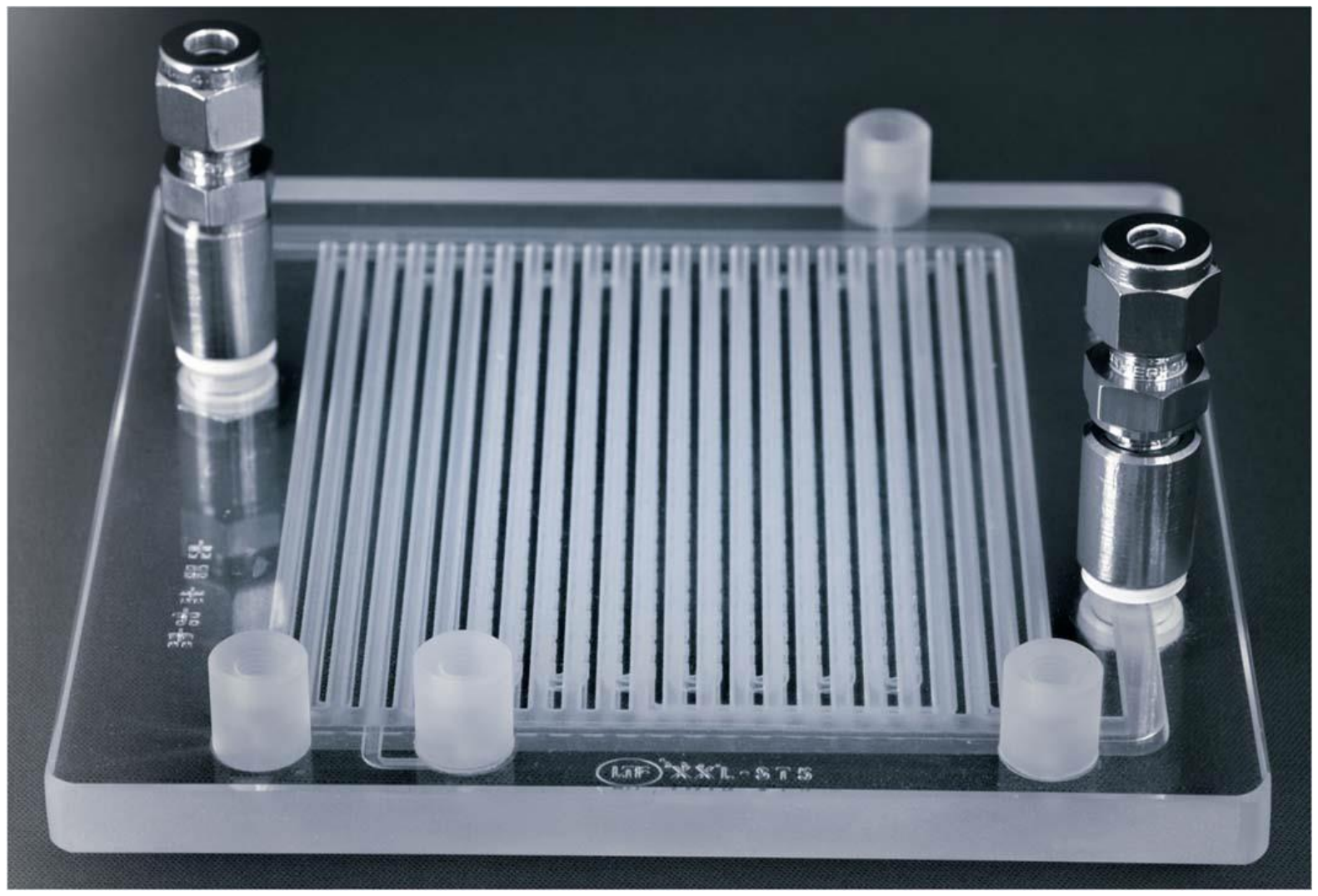

Figure 2: Example of a commercially available glass microreactor. ${ }^{37}$ 


\subsection{Microfluidic Devices and Manufacturing Methods}

Microfluidic devices have been fabricated from a range of different materials including polymers, ${ }^{38}$ glass, ${ }^{39}$ silicon, ${ }^{40}$ metals, ${ }^{4}$ and ceramics. ${ }^{20}$ The selection of material is based on the chemistry required, the operating conditions for the reaction, as well as the ease of manufacture of the part. There are a wide range of different commercial and academic manufacturing procedures used to produce these microfluidic devices. Each method comes with a range of materials, as well as financial and technical considerations. The following subsections outline the techniques most widely used to manufacture these microfluidic devices.

\subsubsection{Glass Microreactors}

In microfluidics, glass is still seen as the gold standard material for the manufacture of chemical reactors due to its excellent thermal conductivity, thermal stability, optical transparency and chemical compatibility. ${ }^{35}$ These microfluidic devices are commercially manufactured to meet a variety of demands ranging from synthetic and analytical devices, to fluidic devices used in biological assays. However, due to the complexity of the manufacturing process they are often highly expensive to purchase and often overlooked for cheaper alternatives. Glass microreactors can be manufactured via a number of different glass patterning techniques. These processes allow microreactors to be produced from a range of different glasses, such as silicon bondable glasses, e.g. Borofloat or Pyrex, or glass with excellent optical transparency such as quartz. ${ }^{41}$

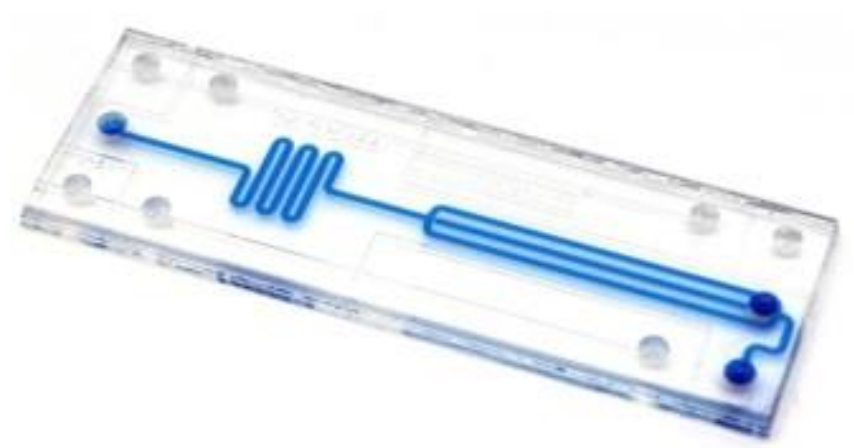

Figure 3: Example of a glass chip reactors. ${ }^{42}$ 


\subsection{1.a. Isotropic Wet Etching of Glass}

The wet etching process begins when a highly polished glass surface is coated with a $100 \mathrm{~nm}$ adhesive layer being applied (usually titanium or chromium based). Typically, an epoxybased photo-resist (a light sensitive material used to form a pattern on a surface), is then spin coated onto the glass sheet in a micron sized film. This photo-resist is exposed through a chemical mask which defines the boundaries of the microreactor channel network. The exposed area of the photo-resist is then removed before subjecting the glass surface to attack from a chemical etchant (Figure 4).

(a)

(b)
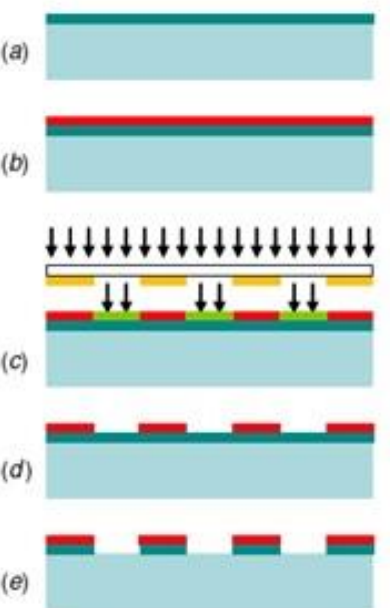

$(f)$

(g)

(h)
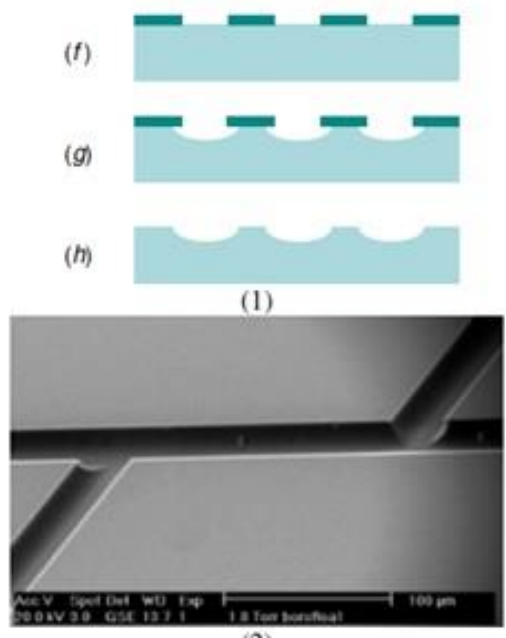

(2)

Figure 4: The chemical etching process: a) application of the adhesive layer; b) application of the photoresist; c/d) exposure of photo-resist; e-h) chemical etching process and removal of remaining adhesive layer; 1) scanning electron microscope (SEM) image of chemically etched channel of $2 .{ }^{43}$

The specific etching solutions depends on the chemical composition of the glass, but typically wet etching of glass is carried out using hydrofluoric acid (HF) as the chemical etchant. ${ }^{44}$ Chemical etching dimensions ranging from 1-300 $\mu \mathrm{m}$ are possible depending on the specific process and resolution requirements, with the etching rate being a parabolic function of $\mathrm{HF}$ concentration (typically $0.5-10 \mu \mathrm{m} \cdot \mathrm{min}^{-1}$ ). The use of wet etching has several limitations, most notably difficulties in controlling the etch profile which can severely limit the possible resolution of channels. This limitation is often caused by the chemical etchant attacking the adhesive layer, which if left for long enough will under-etch the photo-resist and delaminate it from the glass (Figure 5). Slower etches, producing flat channels of only a few micrometres in depth are possible using a buffered $\mathrm{HF} /$ ammonium fluoride $\left(\mathrm{NH}_{4} \mathrm{~F}\right)$ mixture. As glass is an amorphous material, the etchant will typically destroy the two side walls of the 
channel just as quickly as the floor. This phenomenon is termed isotropic etching, whereby wet etched microfluidic channels have dimensions that are twice as wide as they are deep. This phenomenon is said to lead to a low aspect ratio, which defines the ratio of each channel dimension with respect to each other. ${ }^{44}$

(a)

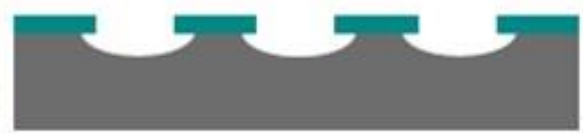

(b)

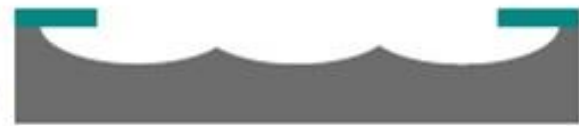

Figure 5: Example of under-etching leading to lower build resolution. ${ }^{43}$

\subsection{1.b. Anisotropic Dry Etching of Glass}

The dry etching process involves exposing a glass surface to a highly ionised gas species. This technique is likened to a micro-scale version of sandblasting, whereby channels are etched using a concentrated stream of sand. In practice, inert ions are generated in a plasma field, and are accelerated by an electrical field created between two electrodes; the glass chip is always fixed to the cathode. The chemical component is the result of the interaction between the radicals generated in the plasma from the reactive gas and the surface of the substrate. This dry etching process is a slow process with etching rates of $0.5-07 \mu \mathrm{m} . \mathrm{min}^{-1}$. The process is also severely limited by the large temperature gradients generated during the etching process, leading to a poor etch selectivity and potential cracking of the glass wafer. Dry etching is generally only favoured when an anisotropic etching profile is required, and consequently high aspect ratio structures are necessary. ${ }^{45}$

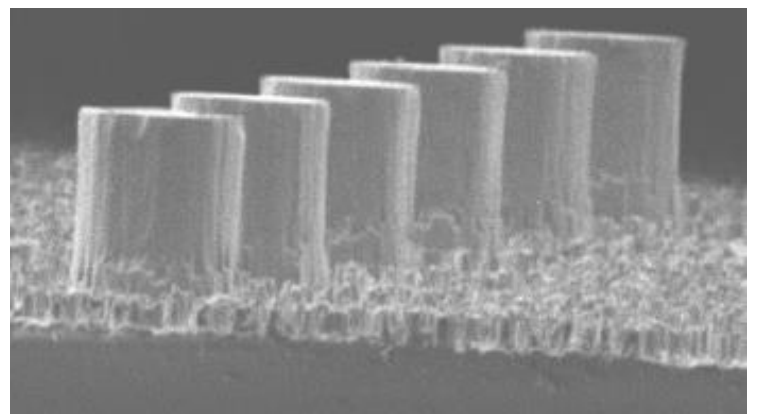

Figure 6: SEM image of glass pillars anisotropically etched into glass. ${ }^{45}$ 


\subsubsection{Silicon Microreactors}

Silicon is also another excellent material for manufacturing microfluidic devices. Due to the well developed field of research of microelectromechanical systems (MEMS), ${ }^{46}$ it is possible to produce very complex 2-D channel networks at a lower cost than those produced from glass. Silicon microreactors also demonstrate excellent thermal conductivity, and are often selected when a uniform reaction temperature distribution is required. ${ }^{44}$ Well established wet and dry etching manufacturing processes enable fabrication of micro channels with controlled sidewall shape and channel dimensions from $\mathrm{nm}$ to $\mathrm{mm}$. A wide range of micron sized flow, pressure, and temperature sensors, have been integrated into these devices. Oxidation of silicon leads to the formation of a glass layer on the surface of the part, so that an oxidised silicon microreactor becomes functionally equivalent to a glass reactor. Silicon microreactor manufacturing processes, like glass, can be subdivided into two categories; dry etching and wet etching. However, unlike with glass reactor manufacture, dry etching is often the process of choice due to its increased manufacturing rates. ${ }^{44}$

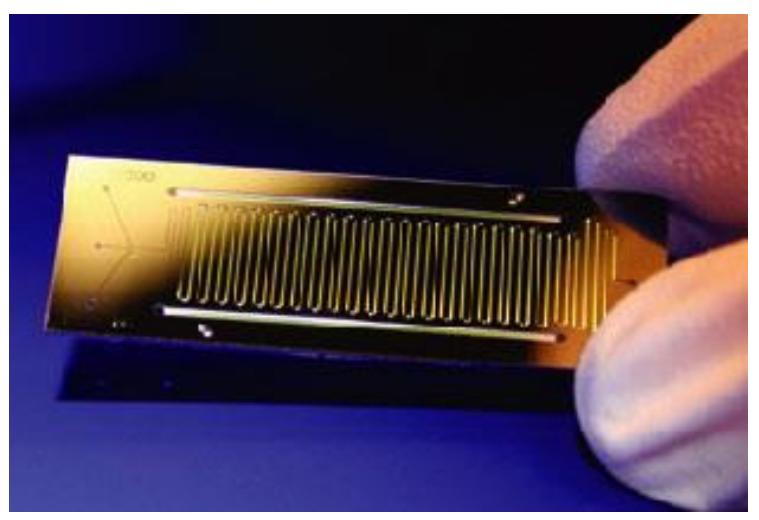

Figure 7: Example of a silicon microreactor. ${ }^{47}$

\subsection{2.a. Isotropic Wet Etching of Silicon}

The wet etching processes used to manufacture silicon microreactors can be defined as either orientation dependent etching, or isotropic etching. The general procedure is largely the same as the wet etching processes used in glass etching; generally, wet etching of silicon is only used when a dry etching process is not available. Isotropic wet etching of silicon is performed using an etching mixture of nitric acid $\left(\mathrm{HNO}_{3}\right), \mathrm{HF}$ and acetic acid $\left(\mathrm{CH}_{3} \mathrm{COOH}\right)$. This is a redox reaction whereby the $\mathrm{HNO}_{3}$ oxidises the silicon surface, whilst the HF dissolved the oxide layer. This method is a highly aggressive etching process, which limits the choice of 
mask available and also strongly affects the selectivity of the process. Orientation-dependent etching of silicon relies on the selective etching of specific crystalline planes, with the method highly versatile depending upon the different silicon wafer being used. It has gained some use due its high etch rates of up to $50 \mu \mathrm{m} \cdot \mathrm{min}^{-1}{ }^{44}$

\subsection{2.b. Anisotropic Wet Etching of Silicon}

Although rare, anisotropic wet etching of silicon is achievable in alkaline solutions such as potassium hydroxide $(\mathrm{KOH})$ or tetramethyl ammonium hydroxide (TMAH). The size and shape of channels attainable using this technique depends on the difference in etching rates for different crystallographic directions. This allows the production of channel networks defined by crystallographic planes of silicon (Figure 8). This method is limited by the range of etching profiles, with simple shapes such as circular channels not possible. ${ }^{43}$

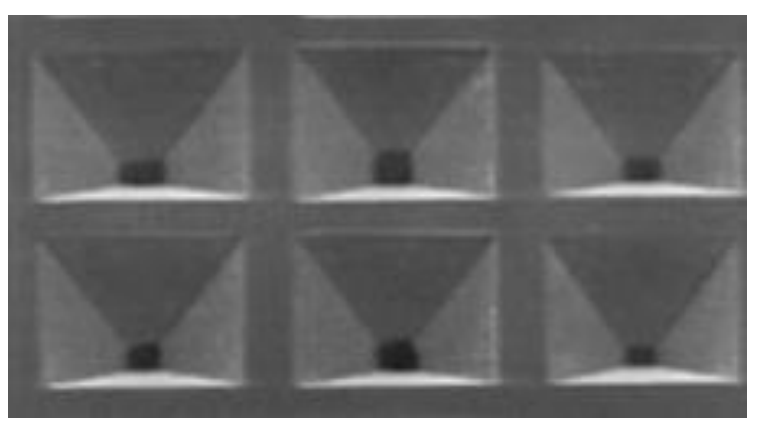

Figure 8: Examples of anisotropic wet etching profiles achieved in silicon. ${ }^{43}$

\subsection{2.c. Isotropic Dry Etching of Silicon}

Isotropic dry etching of silicon is relatively uncommon, however a typical dry etch uses xenon difluoride $\left(\mathrm{XeF}_{2}\right)$, a powerful fluorinating agent, as the precursor. This technique is only applied for dry-release in surface micromachining. ${ }^{44}$

\subsection{2.d. Anisotropic Dry Etching of Silicon}

Two procedures are routinely used in dry anisotropic etching of silicon when aiming to achieve high etch resolutions: the Bosch process and cryogenic etching. ${ }^{48}$ The Bosch process is a two-step method involving alternate passivation and etching steps. The passivation step involves the use of octafluorocyclobutane $\left(\mathrm{C}_{4} \mathrm{~F}_{8}\right)$ based plasma, to generate a PTFE like 
fluoro-polymer over the surface of the silicon. This polymer mask is removed via an "ion bombardment" before being etched using sulphur hexafluoride $\left(\mathrm{SF}_{6}\right)$. Etch rates of up to 30 $\mu \mathrm{m} . \mathrm{min}^{-1}$ can be achieved using this process, as well as a high aspect ratio. ${ }^{49}$ The cryogenic deep reactive ion etching (RIE), cools the silicon chip to $-110^{\circ} \mathrm{C}$, before etching the silica away with fluorine radicals generated using a $\mathrm{SF}_{6} / \mathrm{O}_{2}$ mixture. When using cryogenic RIE, the etching profile is very sensitive to the specific $\mathrm{SF}_{6} / \mathrm{O}_{2}$ ratio. Etch rates of up to $7 \mu \mathrm{m} . \mathrm{min}^{-1}$ can be achieved using this process. ${ }^{50}$ The choice of which process is favoured largely comes down to available equipment, with both achieving excellent etch selectivity.

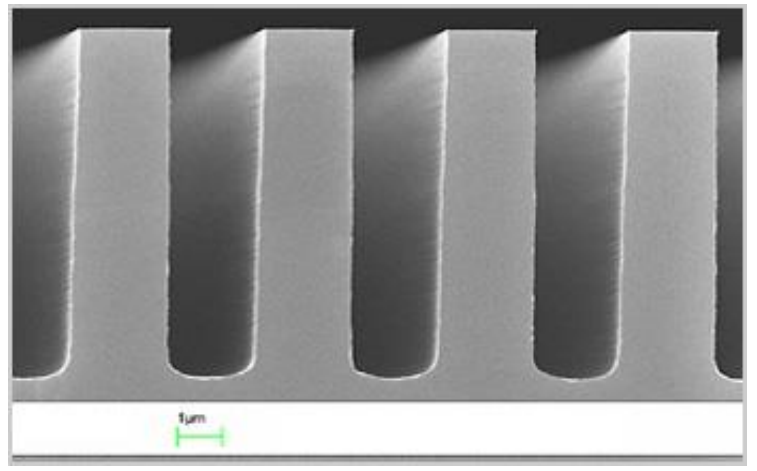

Figure 9: Example of an anisotropic wet etch of silicon via the Bosch process. ${ }^{51}$

\subsubsection{Glass and Silicon Bonding Methods}

Having etched out the requisite 2-D channel network onto the microreactor, it is sealed into place using a second piece of glass or silicon, making the reactor water and pressure tight. Glass and silicon bonding methods suitable for microfluidic applications can be classified as direct, anodic, glass soldering or adhesive. The process used depends on a number of factors including the thermal coefficients of expansion of the materials, surface chemistry, temperature limitations, price, throughput, and yield.

\subsection{3.a. Direct/Diffusive Bonding}

Two highly polished, mirrored glass or silicon surfaces with excellent planarity can be bonded by simple weak van der Waals forces, when placed in contact at room temperature (RT). This process does require a highly efficient cleaning regime to avoid contamination of the surface. This bond strength is relatively weak and can be increased by activation of the two surfaces or annealing of the glass or silicon. The activation process involves heating the 
glass to $\sim 500^{\circ} \mathrm{C}$ or silicon to $\sim 1000^{\circ} \mathrm{C}$, before activating the surface with a $\mathrm{N}_{2} / \mathrm{O}_{2}$ generated plasma source. Fusion bonding of glass can also be achieved by annealing two glass wafers together at $650^{\circ} \mathrm{C}$. The glass must be cooled slowly to prevent cracking of the glass, and the plates must be supported in the horizontal plane.

\subsection{3.b. Anodic Bonding}

Anodic bonding is a process that can bond a glass wafer to a patterned silicon chip. When placed under high temperatures and voltage, sodium ions in the glass wafer migrate towards the negative electrode, causing an irreversible chemical bond between the glass and silicon faces. The strength of this chemical bond is dependent upon the quality of the polished surface, and the cleanliness of the wafer. ${ }^{52}$

\section{Applied Downward Pressure}

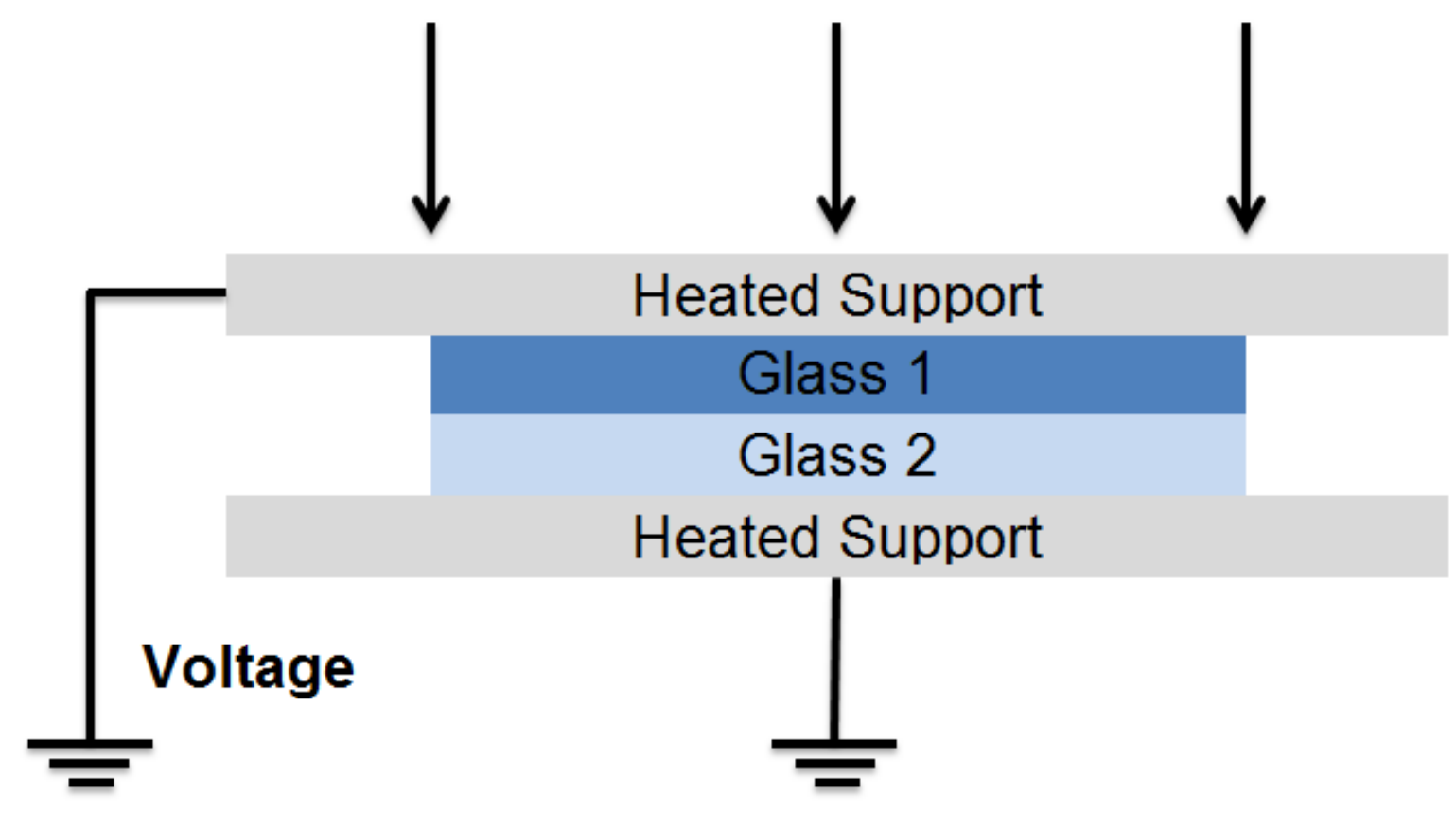

Figure 10: Schematic diagram for the anodic bonding of glass.

\subsection{3.c. Adhesive Bonding}

Adhesive bonding is a low cost, robust, and simple method of bonding together two patterned glass surfaces. Unlike other bonding techniques, the adhesive layer is able to readily conform to any topography at the sealing interface. A range of thermoplastic and thermosetting 
polymers are used as sealants including UV cured epoxy resins, polyimides, benzocyclobutene (BCB), and Parylene C. Parylene $\mathrm{C}$ is favoured due its high chemical and thermal compatibility, as well as its high adhesive properties. BCB is resistant to high levels of acidity and alkalinity, and is also completely UV transparent. Polyimides and UV curable epoxy resins offer less expensive and more readily available adhesive alternatives. The choice of polymer is largely dependent on the specific requirements of each microfluidic chip. $^{53}$

\subsection{3.d. Glass Soldering}

When trying to bond two surfaces that do not have the same thermal expansion coefficient, it may be necessary to use an intermediate glass solder layer. Glass solder is a mixture of low melting point glass and solvent. ${ }^{35}$

\subsubsection{Polymer and PDMS Microreactors}

Polymer microreactors, typically PDMS, have gained widespread use in the world of microfluidics. PDMS has become a prevalent material with which to manufacture microreactors, due to the reduced speed and expense associated with the soft-lithography processes used to manufacture them. ${ }^{34}$ Unfortunately, these polymer devices are at risk of destructively swelling or dissolving in organic solvents and are often over-looked for more chemically stable alternatives. More recent advances have seen more chemically stable fluoropolymers such as FEP utilised, ${ }^{54}$ however the bond strength between glass and fluoropolymers limits these devices to low pressure applications. These soft-lithographic processes are based around rapid prototyping of a positive relief of the channel network, and replica moulding using PDMS.

\subsection{4.a. Rapid Prototyping and Replica Moulding of PDMS}

The rapid prototyping process begins with the creation of a 3D CAD of the part. This image is then printed onto a transparent sheet which serves as the photomask. This photomask is used in contact lithography to produce a positive relief of the photo-resist on the silicon wafer. This positive relief can then be used for the moulding of PDMS devices. The photo- 
resist, when made from a durable photo-curable epoxy, can be reused. The advantages of using rapid prototyping methods like this are a reduction in time and cost for each new design cycle. Unfortunately, poor resolution when producing the photomask can cause an upper limit on the complexity of manufacturable features available further down the manufacturing line. This inhibits the production of the more complex channel networks required in some areas of microfluidics. ${ }^{55}$ The solid relief is then used to cast a negative replica of the relief from PDMS. The part is cured in an oven after casting, with the solid replica being peeled away from the relief. Access holes to the channel network are often produced after this manufacturing process using a borer, with the addition of appropriate threaded channels done via conventional tapping. The part is then adhered to a flat sheet of PDMS to seal the channels. This sealing step occurs as either a reversible seal or an irreversible seal. Reversible sealing is possible due to the flexibility of the polymer, allowing it to conform to minor imperfections in the surfaces. This will generate weak van der Waals forces capable of withstanding low pressures in the channel network. Irreversible sealing of the PDMS parts can be achieved by exposing both surfaces to a plasma source. This aids in the generation of brand new silicon-oxygen-silicon bonds between the layers, leading to a water tight seal capable of withstanding higher pressures up to around 50 psi. As a cheaper alternative, thermoplastic and thermosetting polymers sealants can be used, including UV cured epoxy resins, polyimides, $\mathrm{BCB}$, and Parylene $\mathrm{C} .^{56}$
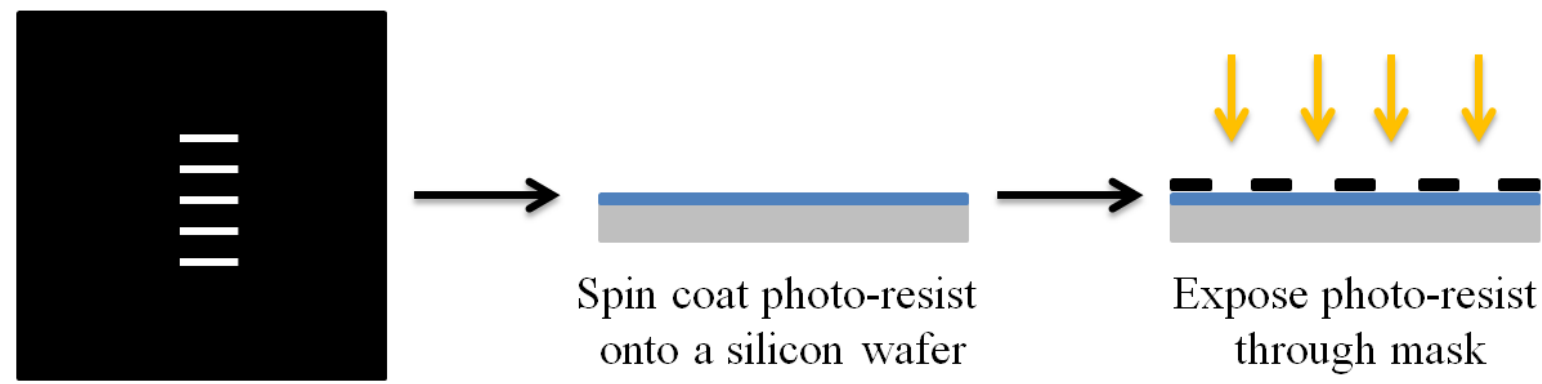

Photomask

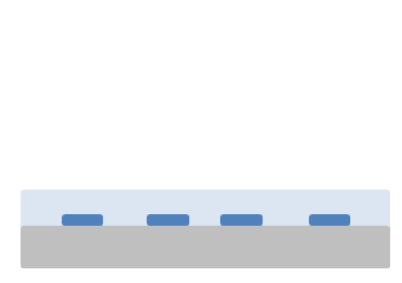

PDMS replica cast

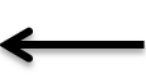

Production of positive relief of pattern

Figure 11: Example replica moulding of PDMS procedure. 


\subsubsection{Other Polymer Microreactors}

Polymer-based microreactors made from inert polymers such as PEEK, are relatively inexpensive and easy to produce. They are produced via a range of well established manufacturing techniques including injection-moulding, reaction injection-moulding, hot embossing, injection compression moulding, and phase-separation-micro-moulding techniques. Many polymers do not demonstrate good solvent or thermal compatibility, and show limited mechanical properties such as discolouration in UV-light and loss of flexibility over time. Thus, the application of these reactors is mostly restricted to aqueous chemistry at atmospheric pressure and temperatures.

\subsection{5.a. Injection Moulding of Polymer Microreactors}

Injection moulding of macroscopic parts is a well known process, and can be adapted to produce micro-scaled chemical reactors. The process begins with the mould cavity (equipped with a micro-structured mould insert) being closed, evacuated, and heated above the glass transition temperature of the specific polymer. An injection unit then heats the polymer, usually in the form of granules, and presses the viscous polymer into the mould. The polymer is subjected to holding pressure to compensate for any material shrinkage. The polymer is then cooled down to below its glass transition temperature and de-moulded from the tool. Injection-moulding is possible using a wide variety of thermoplastic polymers such as polycarbonate (PC), polyvinylchloride (PVC) and PEEK. The process has short production times once the mould insert has been manufactured, but is limited in design complexity by other bulk machining processes required to make the insert. ${ }^{57}$ As well as this the complex thermos-viscoelastic material properties of the polymers used makes the selection of the specific moulding conditions critical to the quality of the final attained part. This is particularly pertinent to the removal of the part from the mould, which limits the specific geometries of the part that can be realised, as well as the material it can be manufactured from. ${ }^{58}$ 


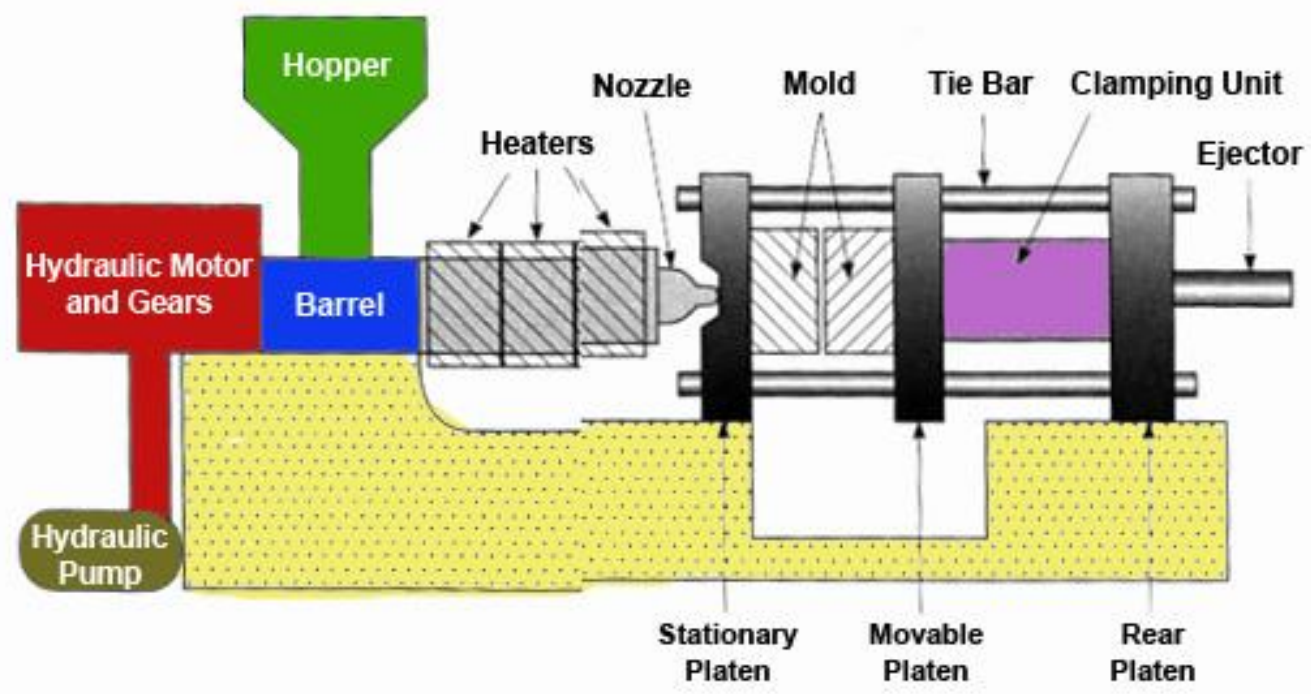

Figure 12: Diagram of the injection moulding process. ${ }^{59}$

\subsection{5.b. Reaction Injection Moulding of Polymer Microreactors}

The reaction injection moulding process is similar to the injection moulding process, however two reactive components are injected into the mould insert together. This allows the production of parts from thermosetting plastics such as polyurethanes, polyesters and polyureas. $^{60}$

\subsection{5.c. Hot Embossing of Polymer Microreactors}

The hot embossing process consists of the following steps: a thermoplastic film is inserted into the micro-moulding machine and heated to above its glass transition temperature, a micron-sized mould insert is pressed into the pre-heated thermoplastic film, the machine is cooled and the insert is removed from the part. The process is once again limited in design complexity by other bulk machining processes required to make the insert. ${ }^{61}$

\subsection{5.d. Injection Compression Moulding}

Injection compression moulding is a hybrid approach of injection and reaction injection moulding, whereby the polymer is injected into the mould insert before being compressed into the insert by a "closing" tool. This process is used to overcome the issues associated with injecting through a narrow cavity for very thin parts. ${ }^{62}$ 


\subsection{5.e. Extrusion of Polymer Reactors}

Extrusion techniques can be used to generate long thin microcapillary tubes through a temperature controlled extrusion nozzle. This type of capillary tube is widely used in a range of industrial applications most notably in HPLC. Typical polymers used include PEEK, PTFE and PFA which are supplied in standard internal tubing diameters of $0.12 \mathrm{~mm}, 0.17 \mathrm{~mm}$, $0.25 \mathrm{~mm}, 0.5 \mathrm{~mm}$ and $1 \mathrm{~mm}$ (amongst others), with the specific reaction conditions being used determining the selection of tubing necessary.

\subsubsection{Metal Microreactors}

Metal microreactors are also commonly used in microfluidics due to the excellent thermal and chemical compatibility of the materials. The range of available materials is considerable, from noble metals such as platinum or palladium, through to more robust materials such as stainless steel (typically 316L), copper, titanium (typically Ti6Al4V), aluminium (typically 6061) and Hastelloy, as well as an extensive list of alloys. The dimensions of stainless steel reactors are generally greater than those used for glass or silicon reactors, with most reactor dimensions being submillimetre, however with the right equipment, micrometre and nanometre geometries are possible. Metal reactors do not allow visual reaction inspection, which can cause problems with contamination and blockages.

\subsection{6.a. Etching of Metal Microreactors}

Wet and dry etching are inexpensive and well established techniques for manufacturing metal microfluidic devices with submillimetre dimensions (Figure 13). The processes are highly comparable to the wet and dry etching techniques discussed previously for glass reactors, with a range of different adhesives, photo-masks and chemical etchants being available.

\subsection{6.b. Micro-machining of Metal Microreactors}

For many metals, chemical etching is not an appropriate manufacturing method, for example noble metals are chemically stable against many corrosive structuring methods. Precision machining processes such as spark erosion and laser machining or more conventional bulk machining methods such as milling and drilling, are commonly used. Equipment and tooling 
can be highly expensive, however the actual manufacturing process can be relatively simple, with many processes being driven by computer aided design (CAD) modelling. Bulk machining processes using milling cutters and diamond profiled cutting wheels, are capable of producing three dimensional features and curved surfaces not possible using other conventional techniques. All of these techniques are ultimately limited by the tooling available, which often restrict channel dimensions to those that are greater than wet/dry etching techniques.

\subsection{6.c. Metal Forming Techniques}

Metal forming techniques, such as embossing, can be a cost efficient method of producing large quantities of metal microreactors, once a suitable negative has been manufactured. Metal forming techniques are highly accurate, and can produce parts with dimensions smaller than $10 \mu \mathrm{m} .^{63}$

\subsection{6.d. Bonding of Metal Microreactors}

Bonding of metal microreactors can be achieved via a number of different processes, including welding, brazing, gluing, diffusive bonding, and soldering. As well as these processes, metal microreactors are also routinely held together using polymer o-rings, metal gaskets and clamps. Diffusive bonding at high temperatures is however often the method of choice, whereby patterned laminas are bonded together at considerable temperatures, pressures and forces. ${ }^{63}$ 


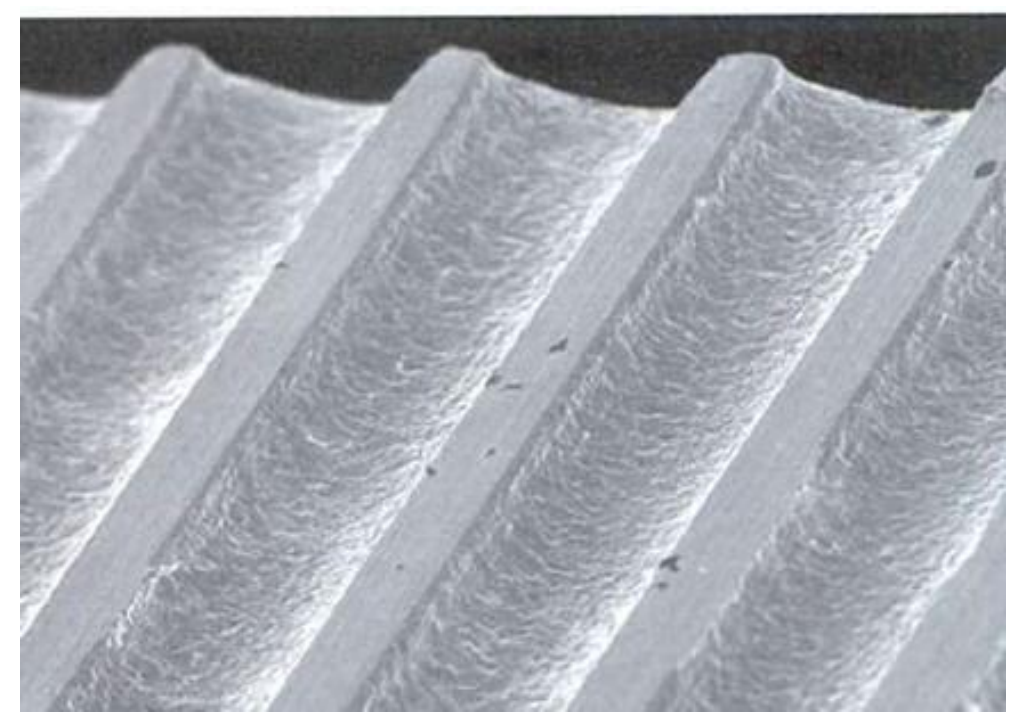

Figure 13: Wet etching of micron sized channels into a stainless steel foil. ${ }^{28}$

\subsubsection{Ceramic Microfluidic Devices}

Ceramic microreactors are often used when metal or polymer microreactors do not have the required thermal or chemical resistance. Unfortunately, the application of ceramic microreactors is limited due to the time-consuming and costly manufacturing processes associated with their production. ${ }^{64}$ Although some machining processes such as laser machining and electrical discharge machining have been applied to ceramic microreactor manufacture, most methods are focussed on moulding in the un-sintered state using a negative mould of the desired part. ${ }^{65}$ High-pressure ${ }^{66}$ and low pressure $^{67}$ are the most common of these methods. Low-pressure moulding (Figure 14), also referred to as hot moulding, is based on the plastification of ceramic powder by the addition of a thermoplastic binder. These binders are often low-melting point waxes or paraffin. This allows moulding at temperatures below $100^{\circ} \mathrm{C}$ and pressures of around $0.2 \mathrm{MPa}$. Consequently, the reduced mechanical and thermal demands allow inexpensive plastic and silicone rubber moulds to be used. ${ }^{68}$ High-pressure injection moulding utilises more expensive metal moulding tools at temperatures of greater than $120^{\circ} \mathrm{C}$ and pressures of around $35 \mathrm{MPa} .{ }^{69}$ 


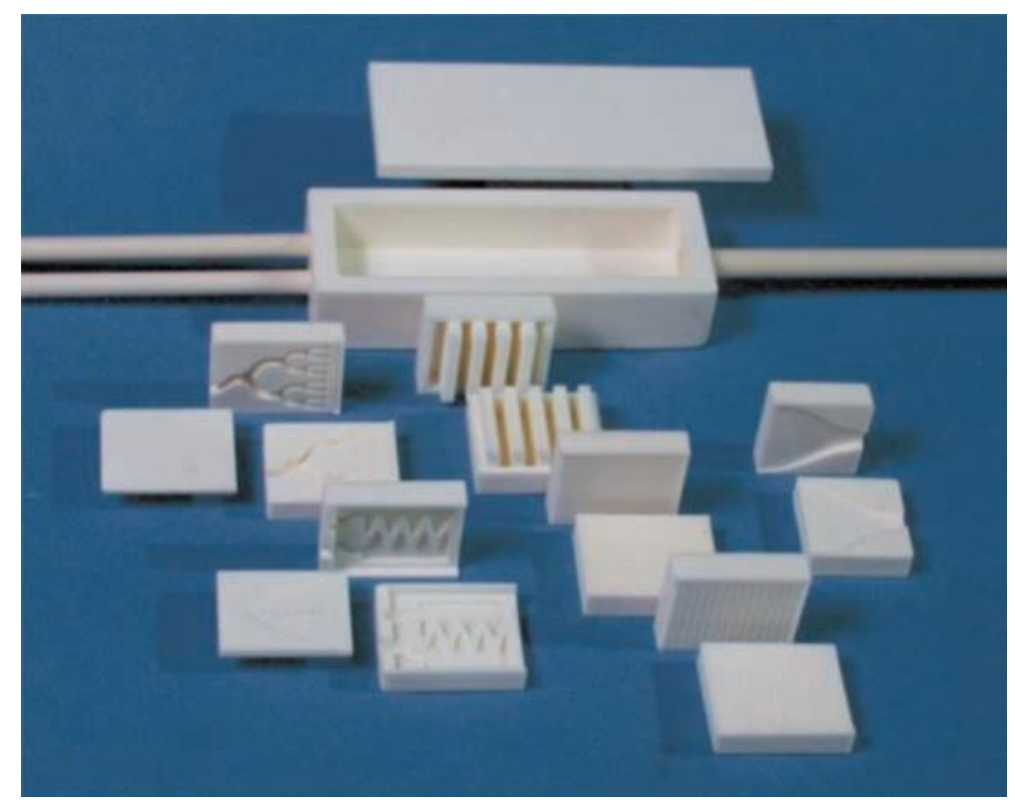

Figure 14: Ceramic microreactors manufactured via low pressure moulding. ${ }^{64}$

\subsubsection{Bonding of Ceramic Microfluidic Devices}

Bonding of ceramic microfluidic devices parts can only be achieved when both materials have similar physical properties, most importantly similar thermal expansion coefficients. This can be achieved via a number of different bonding strategies. Ceramic parts can be fixed together using a firing process, which would bond the parts together irreversibly into a single solid object. Other techniques routinely used are soldering with a sealant such as a glass ceramic composite, clamping of the parts together, or even gluing. ${ }^{63}$ 


\subsection{Microfluidic Design and Micromixing}

Microfluidic design is governed by a wide range of factors including materials, chemical application, cost and functionality. Microfluidic devices can be implemented with a microscale mixer, to provide an intimate contact between the reagent molecules within the fluid stream. Although microfluidic devices are often used as stand-alone devices, they are also incorporated into larger micro total analysis systems ( $\mu \mathrm{TAS}$ ), or as pre-mixers to milli-scale reactors.

\subsubsection{Defining Microfluidic Flow Regimes}

Reagent flow in microfluidic devices can be categorised into two flow regimes: laminar and turbulent. Laminar flow is characterised by smooth and constant fluid motion, whereas turbulent flow is characterised by flow fluctuations and vortices, and is chaotic and unpredictable. ${ }^{70}$ In microfluidic devices laminar flow is often in operation. ${ }^{6}$ Physically, the two regimes differ in terms of the importance of viscous and inertial forces within the system. These forces are measured by the Reynolds number, which will also determine to what extent flow is either laminar or turbulent (Equation 1). ${ }^{71}$

$$
R e=\frac{p u D_{h}}{\mu} \text { or } \frac{u D_{h}}{v}
$$

Equation 1: Reynolds number whereby; Re = Reynolds Number (no units), $p=$ fluid density $\left(\mathrm{kg} / \mathrm{m}^{3}\right), \mathbf{u}=$ fluid velocity $(\mathrm{m} / \mathrm{s}), D_{h}=$ channel diameter $(\mathrm{m}), \mu=$ dynamic viscosity $(\mathrm{kg} / \mathrm{ms}), v=$ kinematic viscosity $\left(\mathrm{m}^{2} / \mathrm{s}\right)$.

At low Re, the viscous effects dominate the inertial forces and a completely laminar flow is said to be in operation. In such a system, the fluid streams will flow completely parallel to one another, with the velocity at any location within the stream being completely uniform. This effect means that mass transfer is only achieved through convective or molecular diffusion between the fluid streams. ${ }^{70}$ At high Re, the flow is dominated by the inertial forces and a turbulent flow regime is in operation. Within this regime, flow is random in both time and space, allowing mass transfer to occur in all directions. Typically, laminar flow will be in operation when the Re is below 1500, and turbulent flow will be dominant when the Re is 
greater than $2500 .^{70}$ There is also clearly a transitional range whereby which regime is in operation is governed by variables such as channel design, dimensions, surface roughness, and aspect ratio. ${ }^{9}$ Microfluidic devices are generally designed with geometries that decrease the mixing path and increase the contact surface area between the different chemical reactants. Micromixer design is therefore either classified as being either active or passive.

Two other non-dimensional fluid parameters commonly used to define flow regimes are the Péclet number and the Strouhal number $(S t)$. The Péclet number is a measure of the relative importance of advection and diffusion in providing the mass transport associated with mixing (Equation 2). ${ }^{72}$

$$
P e=\frac{u L}{D}
$$

Equation 2: Pectlét number whereby; $\mathrm{u}=$ fluid velocity $(\mathrm{m} / \mathrm{s}), \mathrm{L}=$ length $(\mathrm{m}), \mathrm{D}=$ mass diffusion coefficient $\left(\mathbf{m}^{2} \mathbf{s}\right)$.

At high Pectlét numbers advection is dominant, with diffusion taking over at low Pectlét numbers. The Strouhal number is generally associated with active mixing, and represents the ratio between the residence time of a species and the time period of disturbance (Equation 3). ${ }^{73}$

$$
S t=\frac{f D_{h}}{u}
$$

Equation 3: Strouhal number whereby; $f=$ frequency of the disturbance action, $\mathrm{D}_{\mathrm{h}}=$ channel diameter (m), $\mu$ = dynamic viscosity $(\mathrm{kg} / \mathrm{ms})$.

\subsubsection{Passive Mixing}

Passive mixers rely almost entirely on fluidic pumping energy and intricate channel designs, which rearrange the flow in a way which increases the surface contact area between different flow streams. These types of mixers are often a cost-efficient way of inducing efficient mixing into a laminar flow regime. Although a wide range of novel mixing strategies have 
been demonstrated, generally passive mixers can be classified as being either T or Y shape mixers, ${ }^{29}$ serial or parallel lamination, ${ }^{74}$ hydrodynamic focussing of mixing streams, ${ }^{75}$ the introduction of bubbles of gas ${ }^{76}$ or liquid ${ }^{77}$ into the flow, or enhanced chaotic advection using patterned channel walls. $^{78}$

\subsection{2.a. T or Y Shaped Micromixers}

The most simplistic form of micromixers are those that take the form of $\mathrm{T}$ or $\mathrm{Y}$ shaped intersections (Figure 15). ${ }^{79}$

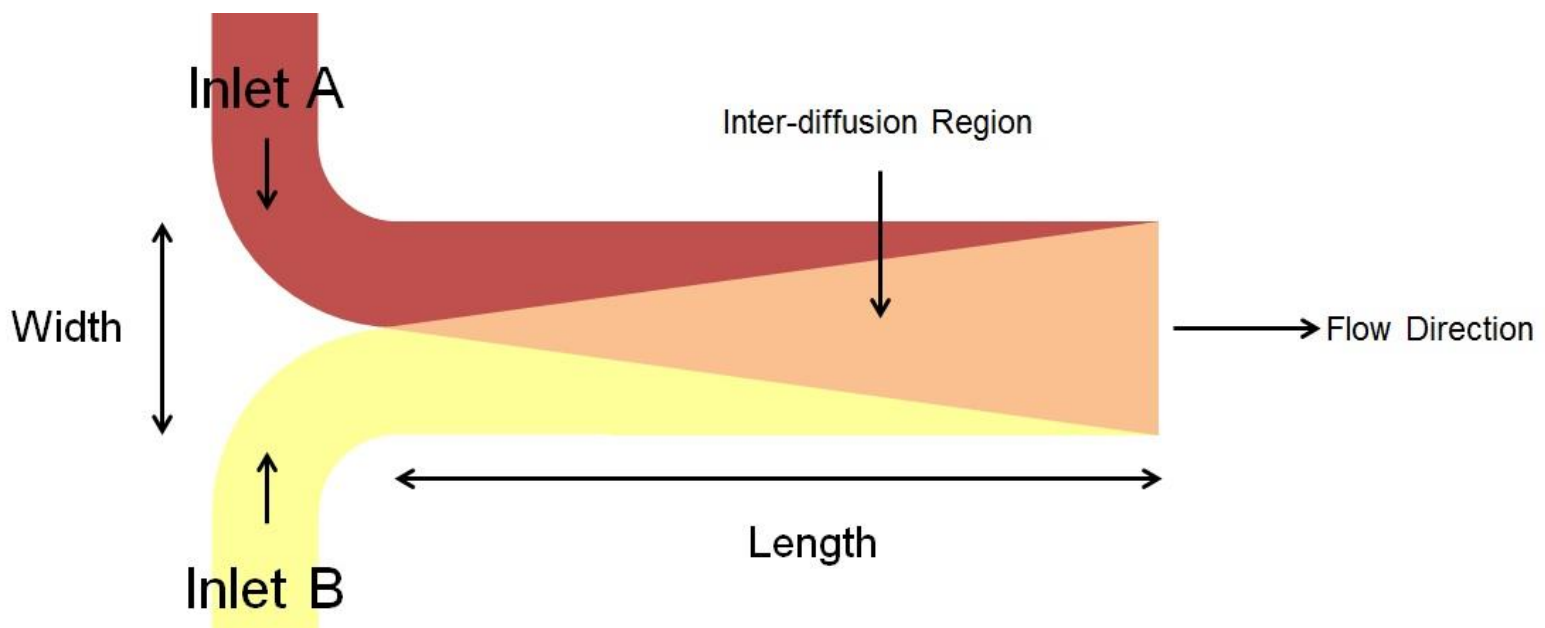

Figure 15: T-shaped micromixer with two input fluid streams.

Mixing within this type of channel design occurs largely via diffusion of the species at the liquid interface, hence mixing efficiency can be relatively low requiring a longer channel design. Mixing efficiency can be improved through the introduction of surface roughness, ${ }^{80}$ higher flow rates, ${ }^{81}$ and narrowing of the mixing channel. ${ }^{82}$

\subsection{2.b. Lamination Micromixers}

Lamination micromixers can be subcategorised into parallel and sequential type mixers. In order to achieve lamination mixing three steps are required: flow splitting, flow recombination and flow rearrangement (Figure 17).

Parallel lamination mixing, splits the main inlet stream into $n$ sub-streams, before re-joining them to form a single laminate stream. This increases the contact surface area between the two streams and enhances the mixing speed of the device by a factor of $n^{2}$. 

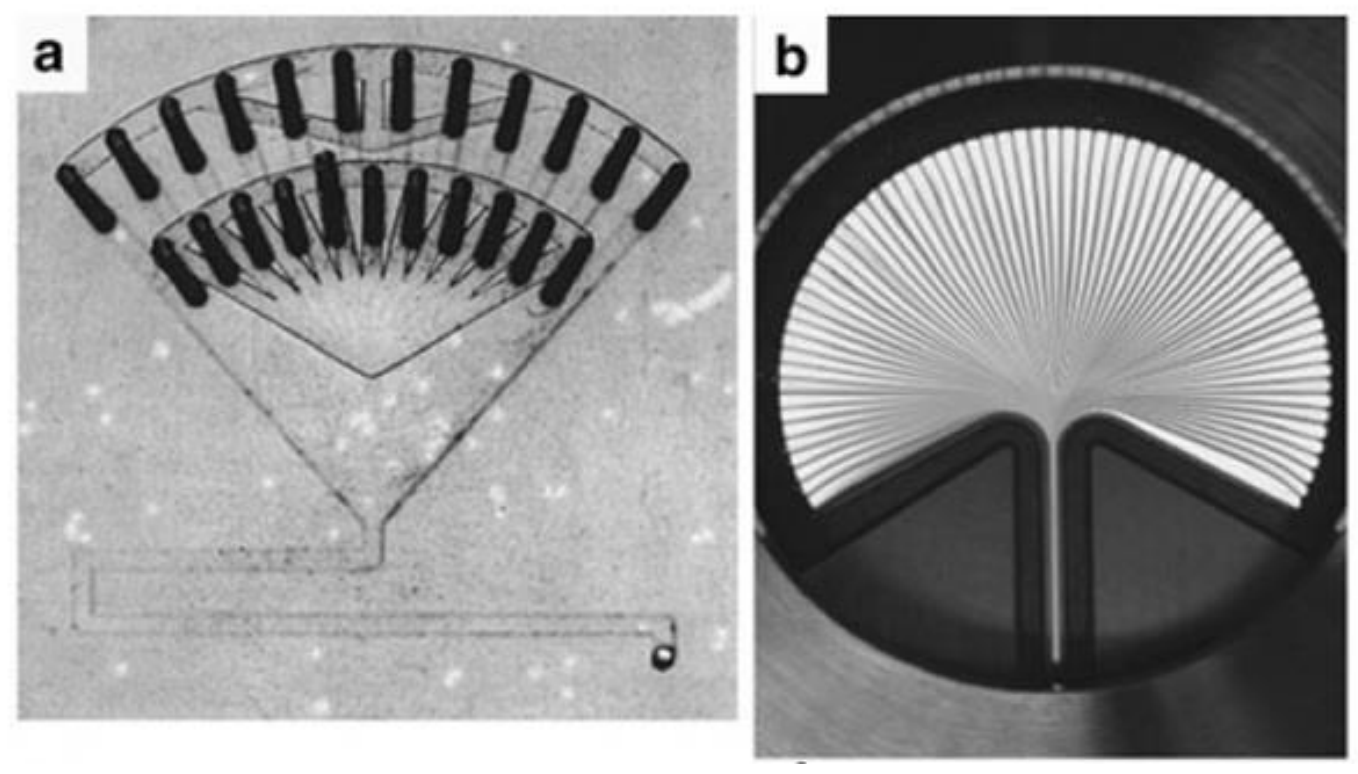

Figure 16: Examples of parallel lamination micromixers. a) bifurcation type mixer; b) interdigital type mixer. ${ }^{9}$

Sequential micromixers, also known as split and recombine (SAR) micromixers, differ in that they rely on sequential splitting and recombining of the flow stream in multiple stages. As with parallel lamination, this relies on the increased contact surface area between the different flow streams, and hence decreases the required path length and overall mixing time. ${ }^{83}$ The designs of SAR mixers differ greatly in the exact geometry by which they actually achieve this fluid arrangement, with fork-like, ramp like, and curved architectures being commonly implemented. In general, SAR type mixers work at very low Reynolds numbers. Figure 17 shows how a laminar flow stream can be sequentially split and recombined twice to create 8 laminar streams. The number of laminar streams created can be calculated using the formula $2^{\mathrm{n}+1}$, where $\mathrm{n}$ equals the number of sequential SAR sections in operation. 


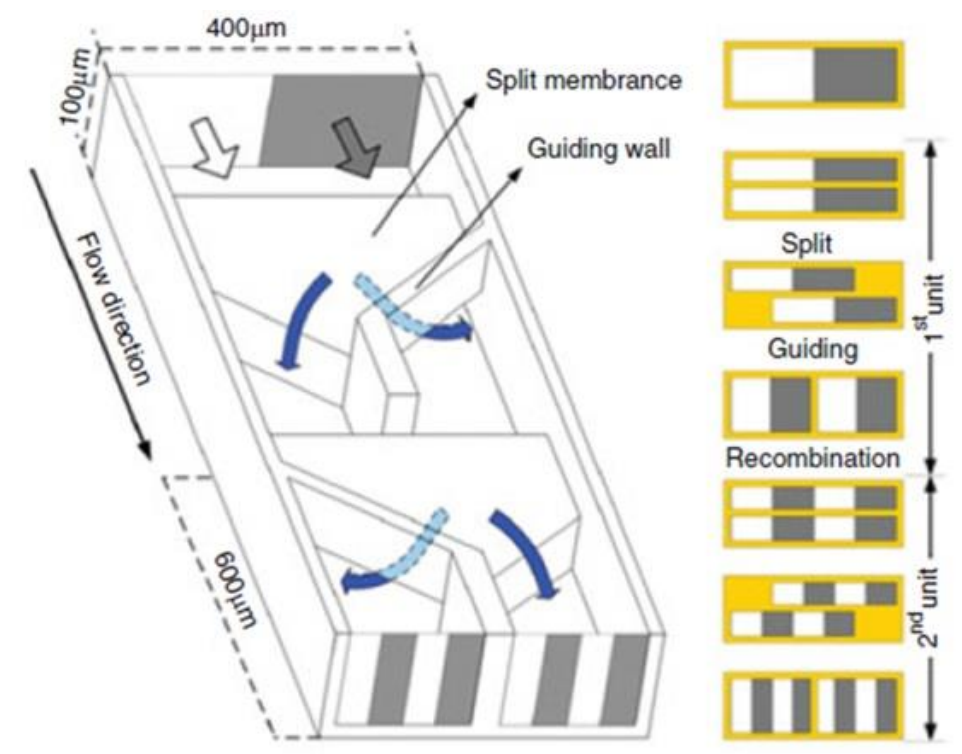

Figure 17: Sequential split and recombine process. ${ }^{83}$

\subsection{2.c. Hydrodynamic Focussing}

The general design for a hydrodynamic focusing mixer is a long microchannel with three inlets. The central flow stream is constrained on both sides via two sheath streams. These sheath streams constrain the central flow to achieve smaller and thinner lamination widths. The extent of this narrowing effect is controlled by the difference between the stream flow rates, with a greater difference leading to a narrower central stream (Figure 18). ${ }^{75}$ This type of microfluidic inlet has a large number of practical applications, however the dramatic increase in contact surface area between the flow streams is the most commonly sought after, as it can substantially increase mixing speeds within a flow system.
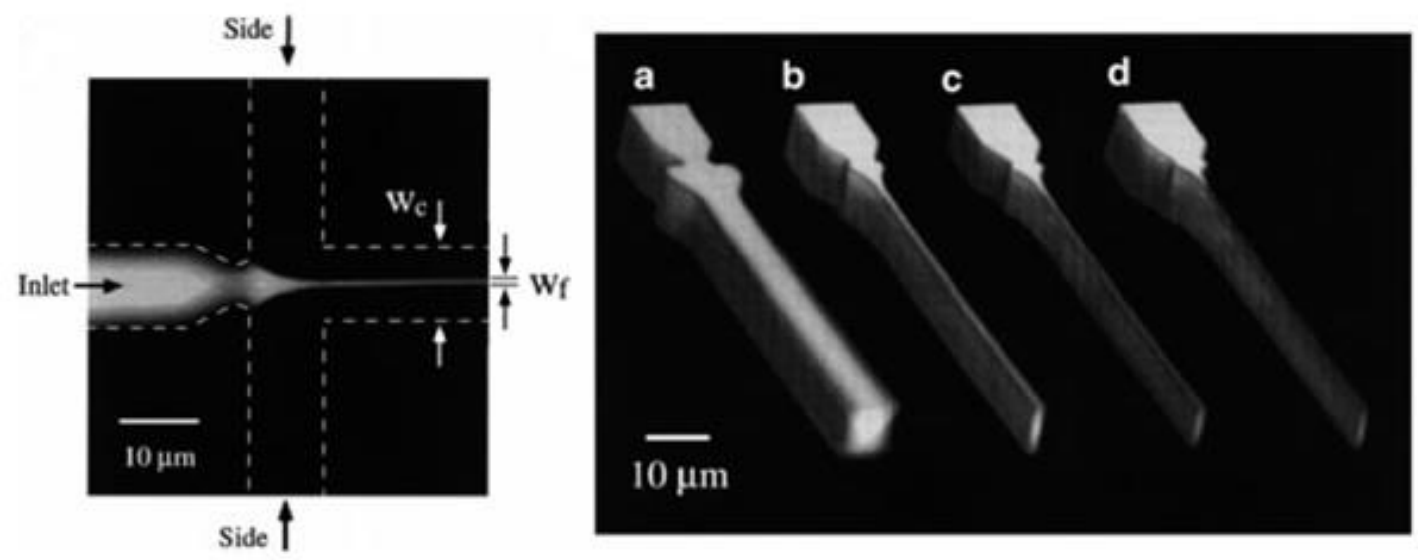

Figure 18: Examples of hydrodynamic focussing mixers. ${ }^{75}$ 


\subsection{2.d. Chaotic Advection Micromixers}

Advection can be defined as the transport of a substance within a moving fluid. In the mixing strategies discussed previously, this advection occurs in the direction of the flow, and therefore has no effect of the transport of the substance. It is possible however to introduce chaotic advection within the flow stream, leading to transverse mass transport within the flow. ${ }^{84}$ This transverse flow can be generated in a number of manners such as inserting obstacles into the flow, ${ }^{85}$ and using curved channels, ${ }^{86}$ or convoluted channels. ${ }^{87}$ This turbulence increases the contact surface area between the different flow streams and increases mixing efficiency.

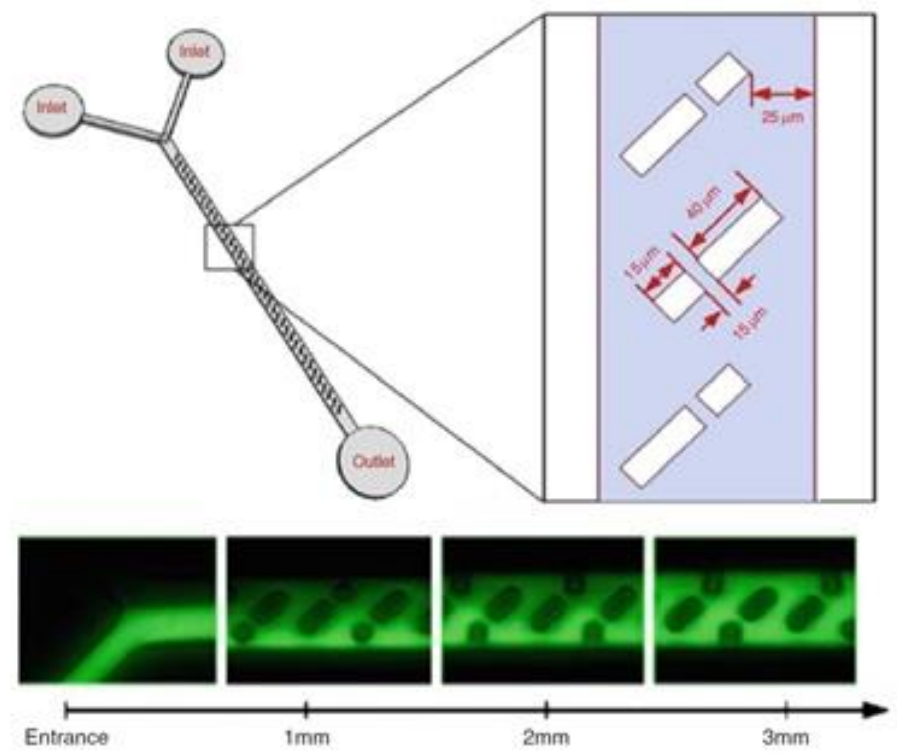

Figure 19: Example of a chaotic advection micromixer. ${ }^{9}$

\subsection{2.e. Microdroplet-based Mixers}

In microfluidic devices it is possible to generate multiphase flows, where two or more immiscible flow streams come into contact with one another. This multiphase flow can take the form of either droplets, slugs or stratified flows. ${ }^{88}$ The capillary number $(\mathrm{Ca})$ is a dimensionless parameter that expresses the relationship between the viscous and interfacial forces within a multi-phase flow stream. $\mathrm{Ca}$ can be defined as being the ratio between these forces (Equation 4); 


$$
C a=\frac{\mu u}{\gamma}
$$

Equation 4: Capillary number whereby: $\mu=$ viscosity of the continuous phase $(\mathrm{kg} / \mathrm{ms})$, $u$ is the average flow velocity $(\mathrm{m} / \mathrm{s})$, and $\gamma=$ the interfacial tension between two fluid phases $\left(\mathrm{kg} / \mathrm{s}^{2}\right)$.

Droplet formation will be seen when interfacial forces dominate and the $C a$ is low. Generally, channel designs are based around either flow focussing or T shaped mixers. In the T-shaped channel design the droplet is generated at the point of contact between the two flow streams, whereas in the flow focussing mixers the droplet is generated when the central flow stream becomes so narrow that the pressure and shear stress exerted on the fluid causes it to collapse and form a droplet.

(a)

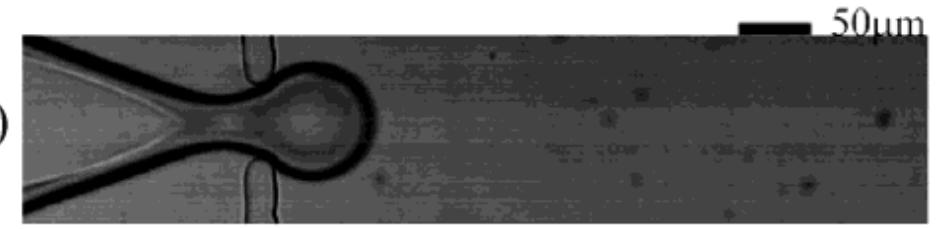

(b)

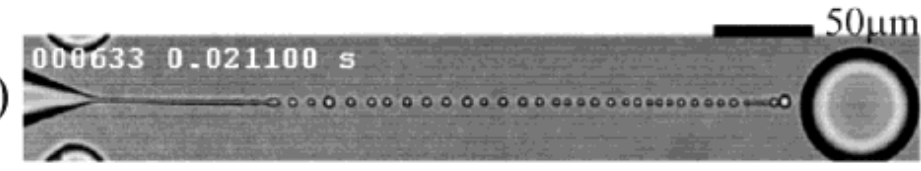

(c)

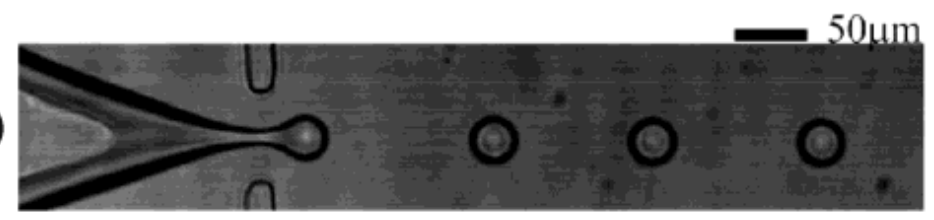

(d)

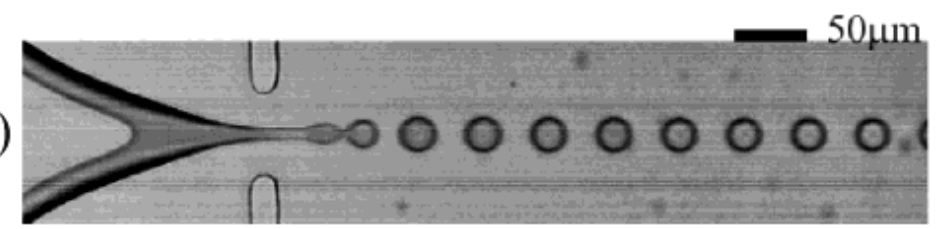

Figure 20: Examples of microdroplet formation. a) squeezing, b) tip streaming, c) dripping and d) jetting. ${ }^{89}$

\subsubsection{Active Mixing}

Active micromixers use external energy inputs to introduce fluctuations that disrupt the fluid flow for accelerated mixing efficiency. ${ }^{90}$ These mixers can be categorised as being either pressure field driven, ${ }^{91}$ acoustic driven, ${ }^{92}$ temperature induced, ${ }^{93}$ electrically induced, ${ }^{94}$ or magneto-hydrodynamic. ${ }^{95}$ Active mixing often leads to more efficient mixing within the device, however the integration of such peripheral devices into microfluidics parts is a time 
consuming and often expensive process. As a consequence active mixing is rarely used for chemical flow applications.

\subsection{3.a. Pressure Field Driven Mixers}

Active mixing can be achieved by inducing a pressure field disturbance within the flow stream. Examples of how this effect can be achieved include integrating micro pumps which drive and stop the flow to create segmentation, ${ }^{96}$ and periodically alternating the flow rate within a channel from high to low in a pulsating manner. ${ }^{91}$

\subsection{3.b. Electrokinetic Disturbance}

Electrokinetic mixers take advantage of fluctuating electrical fields to induce mixing within the microfluidic pathway. This fluctuation can lead to rapid stretching and folding of the fluid interface, effectively stirring the laminar flow regime and inducing efficient mixing. ${ }^{97}$

\subsection{3.c. Dielectrophoretic Disturbance}

Dielectrophoresis is the phenomenon by which polarisation of a particle is induced by a nonuniform electric field. In flow these polarised particles will move away or towards the electrical field, creating chaotic advection and mixing of the fluid. ${ }^{98}$

\subsection{3.d. Electrowetting}

Droplet formation can be induced within a flow channel by electrowetting. Electrowetting relies on the control of the interfacial tension of a droplet using an electric field, allowing droplets of different phases to be coalesced and mixed. ${ }^{94}$

\subsection{3.e. Magneto-Hydrodynamic Disturbance}

Magneto-Hydrodynamic Disturbance (MHD) relies on the coupling of electrodes deposited into the channel wall and an external magnetic field. This allows the induction of a Lorentz 
body force within an electrolyte solution, leading to deformations and stretching of the material interface and enhanced mixing. ${ }^{95}$

\subsection{3.f. Ultrasound Disturbance}

Highly efficient mixing can be achieved by means of acoustic stirring created by acoustic waves. Ultrasonic waves are introduced into channels via integrated piezoelectric ceramic transducers. This leads to acoustic stirring in the direction perpendicular to the fluid flow stream, leading to enhanced mixing within the flow channel. ${ }^{92}$

\subsection{3.g. Thermal Disturbance}

Thermal disturbance is achieved when an increase in the diffusion coefficient due to temperature, increases enhances microfluidic mixing. ${ }^{93}$

\subsection{3.h. Microfluidic Stirring}

Microfluidic stirring can be achieved via the integration of moving parts or stirrer bars into the microfluidic channel. These devices allow rapid mixing of large sample volumes and are applicable to a wide range of different fluids. ${ }^{99}$ 


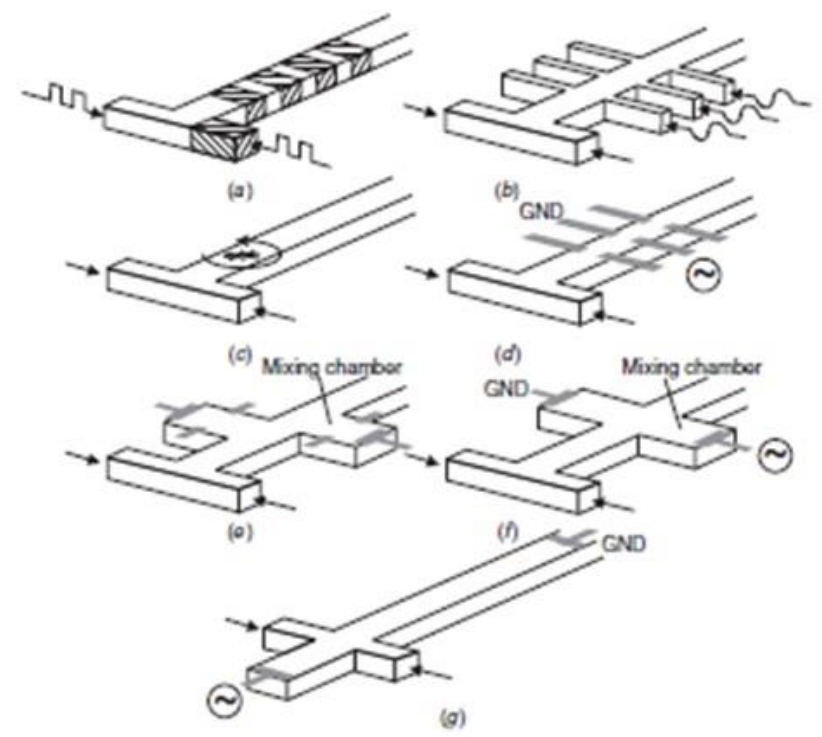

Figure 21: Examples of active mixers: a) serial segmentation; b) pressure disturbance; c) integrated microstirrer; d) electrohdrodynamic disturbance; e) dielectrophoretic disturbance; f and g) electrokinetic disturbance. ${ }^{33}$ 


\subsection{Integration of Spectroscopic Measurements into Microfluidic Devices}

Integration of chemical analysis methods and physical sensors into microfluidic devices allows the user to perform inline reaction optimisation, as well as detailed studies governing reaction mechanics and kinetics. ${ }^{40}$ These inbuilt chemical sensors can be integrated with computer controlled pumps and heating mantles allowing some control over reaction conditions, substantially reducing the time and material costs in identifying optimised processing parameters, and hence accelerating process development. ${ }^{100}$ This opens up faster and more reliable analytical pathways than traditional "black box" approaches, whereby products are analysed off-line. This is particularly true of large industrial scale processes, whereby the technology allows the adoption of a "numbering up" approach. It is important to be able to monitor the output of each unit within the chain, to identify faulty units, or variable outputs during the process. ${ }^{101}$ In line analysis also allows precise control over reaction conditions such as flow regimes, mixing efficiency, temperature distribution etc., as well as revealing the presence of short lived reaction intermediates which may not show up during off-line analysis. Specific techniques for monitoring species concentrations including fluorescence, ${ }^{102}$ UV-Vis, Raman ${ }^{101}$ and Fourier transform infrared (FTIR) spectroscopy, ${ }^{103}$ as well as nuclear magnetic resonance (NMR) spectroscopy, have all had miniaturised analysis systems developed for microfluidic applications.

Fluorescence spectroscopy has gained wide-spread use in $\mu$ TAS due to its high sensitivity to the small detection volumes used in microfluidic devices. ${ }^{104}$ It is based on the detection of light emission from excited singlet states of fluorescent substances (fluorophores). Unfortunately, the majority of pharmaceutical analytes do not fluoresce and therefore have to be chemically altered to do so. This type of spectroscopy also has significant applications in the analysis of biochemically important materials such as amino acids, which can be readily derivatised with a fluorescent tag. A wide range of light sources including lasers, light emitting diodes (LEDs), and lamps can be used as excitation sources. In general, microreactors manufactured from glass or quartz are desirable due to their weak fluorescence backgrounds, however their costly fabrication processes often leads to the use of PDMS devices, whereby the fluorescent background interference must be mitigated for. Detection is usually carried out using an external focussing lens and photomultiplier type detector. A more efficient method of detection can be the integration of optical fibres directly into the fluidic 
microchannel, ${ }^{105}$ or the incorporation of planar waveguides that intersect the flow channel giving direct excitation of the analyte (Figure 17). ${ }^{106}$

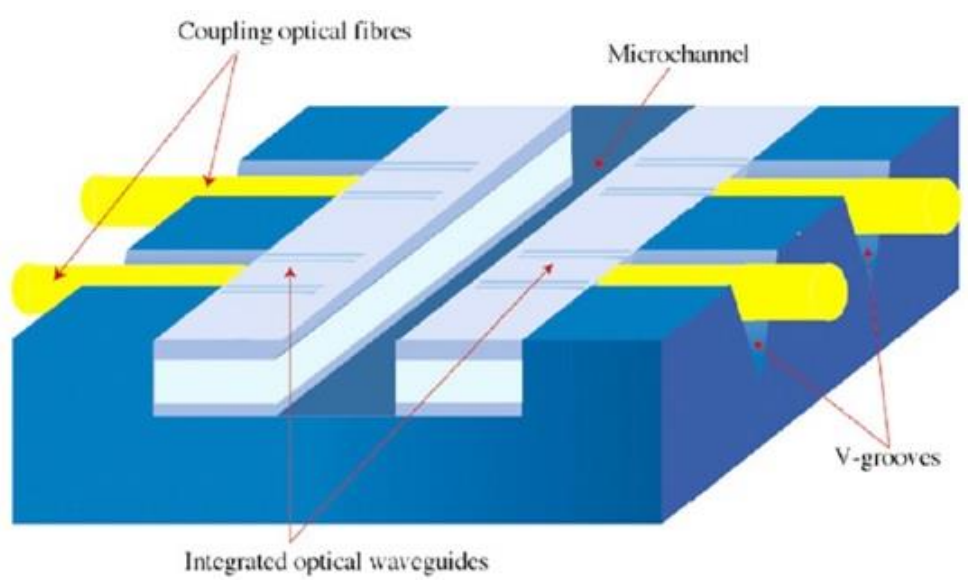

Figure 22: Incorporation of planar waveguides into a microfluidic device for fluorescence spectroscopy. ${ }^{101}$ UV-Vis absorption is a well-established detection method, whereby the absorbance of the incident light source by certain functional groups in the sample molecule is expressed as a function of wavelength and intensity. The simplest way to monitor UV-Vis absorption in flow is via an integrated external flow cell. These flow cells are commercially available and reasonably inexpensive, however it is not the most effective way to take spectroscopic measurements of a flow stream. One approach is to integrate two optical fibres in perpendicular configuration across the flow path, usually via the use of integrated quartz windows (Figure 23). This does however require a delicate and highly precise alignment process. ${ }^{101}$
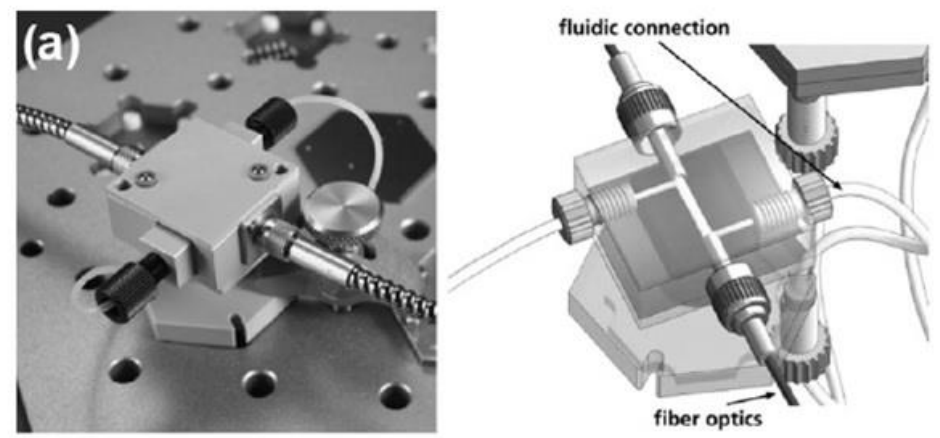

Figure 23: Integrating optical fibres directly into a microfluidic flow path. ${ }^{101}$

IR spectroscopy is the measurement of absorption or emission of electromagnetic radiation in the IR region as a consequence of vibration within a molecule. ${ }^{101}$ FTIR is an effective method for determining chemical structures, functional group conversions, and product concentrations. Consequently, FTIR has been implemented into $\mu$ TAS for a variety of 
applications. ${ }^{107}$ In general, these approaches have been achieved via the capping of microfluidic channels with IR transparent materials, or via off chip FTIR detection. Attenuated Total Reflection (ATR) spectroscopy has also been implemented, particularly when the flow channel path length becomes too large, leading to solvent peaks swamping the spectra. ${ }^{104}$ Inline measurements for IR are usually conducted by placing the microreactor into a commercial IR spectrometer sample compartment, or in a microscope stage connected to an FTIR spectrometer system. ${ }^{108}$ The transparency of glass and silicon to FTIR radiation means these are ideal materials with which to manufacture microfluidic devices for FTIR analysis. IR measurements can also be taken via the attachment of an inline IR flow through cell, often implemented when using non IR transparent materials such as PDMS. ${ }^{109}$

Raman spectroscopy relies on the measurement of inelastic scattering of monochromatic light upon interaction with a flow stream. The spectrum of the scattered light provides information about characteristic vibrations for each molecule, providing a unique "fingerprint" for each compound, and can be utilised for the identification of both organic and inorganic molecules. Unfortunately, like FTIR, the sensitivity of Raman spectroscopy is relatively low. Similarly to FTIR, Raman measurements are usually conducted by placing the microreactor into a commercial Raman spectrometer sample compartment or in a microscope stage connected to a Raman spectrometer system. Measurements can also be taken via the attachment of an inline Raman flow through cell. ${ }^{110}$

NMR spectroscopy is the measurement of absorption and emission of an oscillating radio frequency $(\mathrm{RF})$ that interacts with the atomic nuclei of the compound immersed within a strong external magnet. NMR is widely regarded as the most powerful analytical tool for analysing organic compounds, due to the wide range of structural information that it can provide. Microreactors can be integrated to a conventional NMR instrument via the use of an inline NMR flow probe. ${ }^{111}$ These probes generally suffer from lower signal intensity than a standard NMR sample tube, but are generally strong enough to gain sufficient data to successfully monitor reactions. 


\subsection{Mesofluidic Devices}

Fluidic devices with channel dimensions in the range of $500 \mu \mathrm{m}$ and a few millimetres are termed "mesofluidic" or milli-scale. Mesofluidic technologies which flow reactants through 3-D channels are often referred to as "coil reactors" or "reaction coils". These coils often take the form of lengths of tubing with internal diameters of either 0.5 or $1 \mathrm{~mm}$. Any suitably inert tubing can be used, but generally stainless steel or PTFE are the materials of choice. ${ }^{5}$ Millimetre scale mimics of many microfluidic devices are also not uncommon, however with increasing internal reactor geometries, there is an exponential reduction in control over the reaction environment. Coil reactors typically have millilitre scale internal volumes and therefore allow a much longer residence time than chip reactors, however these smaller dimensions, along with a lack of micro-mixer technology, leads to lower mixing efficiency and consequently slower reactions times. Using a chip reactor as a pre-mixer to your coil reactor will help improve mixing efficiency, aid heat transfer, and lead to a faster and cleaner reaction. Coil reactors traditionally come as either heat resistant polymer or metal tubing; metal reactors (typically stainless steel, aluminium or Hastelloy®) demonstrate good thermal conductivity and chemical compatibility, whereas polymer reactors (PTFE, PEEK, etc.) are not always as reaction friendly. Polymer reactors can also suffer from swelling and discolouration over time, however they are often either transparent or translucent and therefore allow visual inspection of a reaction. Both polymer and metal tubing are inexpensive and commercially available, and reactors of any length, diameter or volume can be manufactured to meet the individual requirements of the reaction.

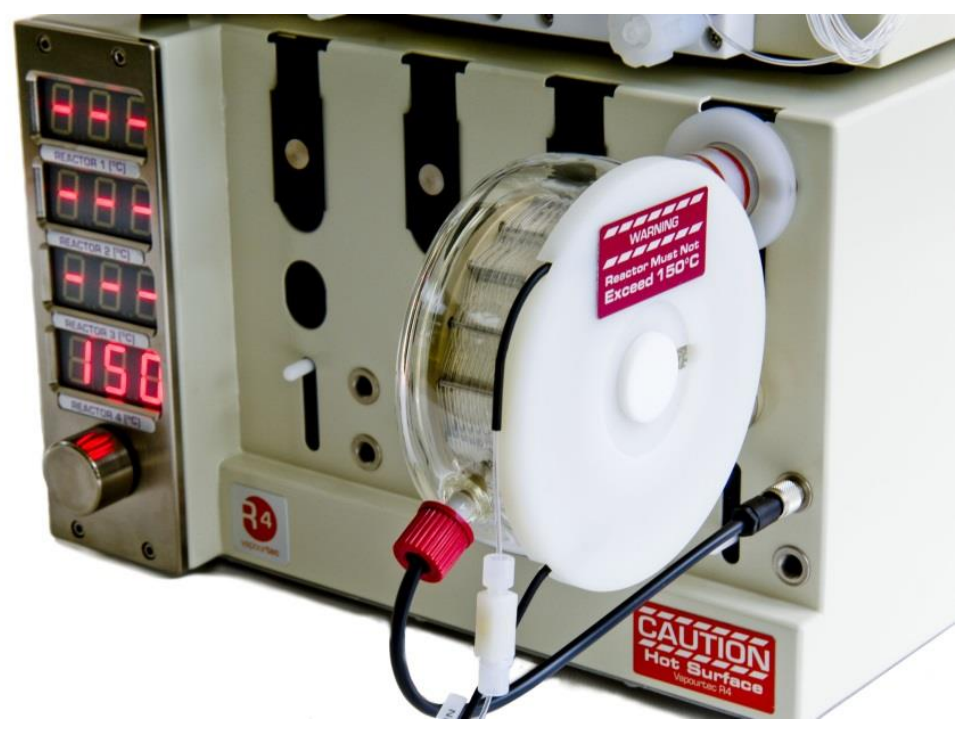

Figure 24: Example of a PFA mesofluidic reactor coil. ${ }^{112}$ 


\subsection{Additive Manufacture}

During the course of this thesis, the research will explore the use of additive manufacturing (AM) as a method of fabricating millilitre-scale flow reactors, to allow the incorporation of the innovative designs which have previously been limited to microlitre-scale microfluidic devices. Developed from rapid prototyping, additive manufacturing, also known as additive layer manufacturing or ' $3 \mathrm{D}$ printing', is the umbrella term used to cover a variety of technologies that can directly produce complex three dimensional parts, with near-complete design freedom. The term additive manufacturing is now widely accepted and is recognised by international standards. ${ }^{113}$ In AM, parts are built layer-by-layer, using techniques such as extrusion, jetting, powder sintering, or photo-polymerisation. Parts can be made with complex and customisable geometries, including those difficult to make by conventional manufacturing techniques. Although still a rapidly developing area of research, AM already offers a range of manufacturing techniques capable of meeting many of the design criteria previously outlined.

The design freedom offered by AM can allow the production of bespoke reactors with near complete design control over reactor path length and internal reaction volume, and therefore the reaction residence time possible for each device. More complex internal geometries such as passive and active mixers are also well within the scope of AM. Commercially, AM can also offer considerable benefits in terms of build cost and production speed. AM manufactured parts can often be taken from concept to realisation within hours, with huge time-savings possible in both the design and manufacturing processes. Manufacturing costs for complex parts such as miniaturised reactor systems can be substantially reduced, with the cost of production directly related to the volume of material used, rather than the necessity for specialist tooling and labour.

However, using AM to produce parts for chemical applications also poses some fundamental challenges. Although the range of materials AM offers is growing, most AM processes are dedicated or limited to specific material types. For instance, glass has long been viewed as the gold standard material choice for chemical reactor manufacture, both in batch and flow processing. Glass is inert to almost all chemical reagents, thermally conductive and stable at high temperatures, transparent to allow reaction visualisation, easy to clean and most importantly trusted industrially. Selective laser sintering (SLS) of borosilicates and glass 
ceramic composites have been successfully demonstrated, however parts often demonstrate poor geometrical resolution, cracking due to thermal shock in the cooling phase and discoloration. ${ }^{114}$ More recent work out of MIT has seen significant improvements in many of these areas, however the achievable geometries are still an order of magnitude greater than those required for microfluidics. ${ }^{115}$ So for the foreseeable future AM must utilise a range of different materials to solve the materials challenge presented by chemical applications. In addition to material limitations, each AM technique has differing constraints for allowable part geometries, accuracy, and spatial resolution. For a given reactor design, this will lead to trade-offs in the selection of AM process and material.

The AM build process begins with the generation of a 3D CAD model of the desired part. The model is then converted into an STL (surface tessellation language) file. The STL file format has become the 3D printing industries standardised data transmission format, allowing transfer of designs between different processes. The STL file format approximates the surface of a model as a triangular mesh, with more complex designs requiring a larger number of triangles to represent the surface. The file is then sliced into sections, each with the information required to print a single layer of the desired part. This file is then uploaded onto the specific AM machine software, the machine build parameters are defined by the user, and finally the part is built. Most AM processes involve post processing such as the removal of un-sintered powder or un-cured resin, the removal of support structures and the removal of the part from the build plate.
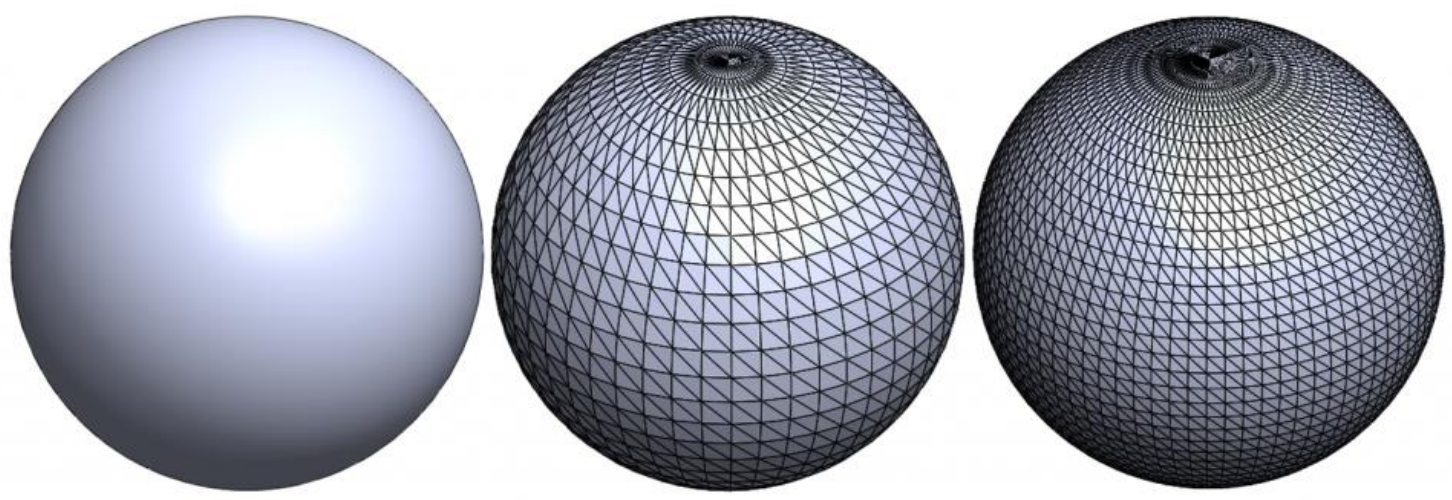

Figure 25: Example of the STL file format representing the surface of a sphere.

This research will look at the applicability of five common AM techniques to millilitre-scale reactor fabrication: stereolithography (SL), ultrasonic additive manufacture (UAM), selective 
laser melting (SLM), selective laser sintering (SLS) and fused deposition modelling (FDM). The strengths and weaknesses of each process have been considered, whilst highlighting the limited prior work in the application of AM to reactor design. The strengths and weaknesses of existing micro-scale and nano-scale reactor manufacturing technologies are also discussed, and ways in which innovative additive manufacturing techniques could be complementary to the world of lab-on-a-chip. 


\subsection{Additive Manufacture of Fluidic Devices}

The first example of microfluidic channel networks being additively manufactured was presented by Lewis et al. in 2003. ${ }^{116}$ The group used an additive process known as direct write assembly to fabricate a network of smooth cylindrical channels $(\sim 10-300 \mu \mathrm{m})$. The direct write assembly manufacturing process begins with the deposition of an organic ink onto a moving X-Y build platform. As each predefined layer is deposited, the extrusion nozzle which is mounted on the $\mathrm{Z}$ axis stage is raised, allowing each subsequent layer to be deposited. Having generated the 3D construct the part is filled with an uncured low viscosity resin. This resin is polymerised and the ink scaffold is removed, leaving the direct negative of the deposited ink filaments prior to the matrix infiltration. ${ }^{117}$ The group used this technique to generate a network of channels which promoted chaotic advection within the flow stream, via vertical obtrusive towers within the flow path.
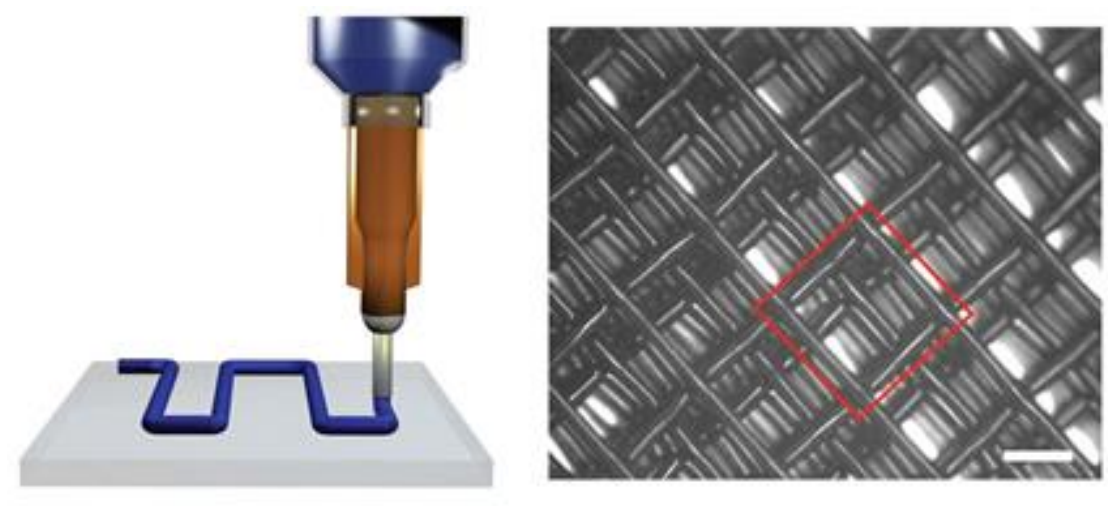

Figure 26: Micro-scaffold fabrication via direct-write assembly. ${ }^{116}$

More recently, the use of AM as a novel process for the production of bespoke fluidic devices has been successfully demonstrated by a number of research groups. Research at the University of Glasgow has seen a number of milli-scale fluidic devices manufactured for continuous flow processes; these devices have all been produced via the fused deposition modelling (FDM) process. The trademarked FDM process was developed and commercialised by Stratasys in 1990, and has become the market leading 3d printing process for the AM of fluidics. FDM uses a temperature controlled extrusion nozzle to deposit a viscous molten thermoplastic polymer into a three-dimensional structure. This process can be used with a range of commercially available thermoplastic polymers including acrylonitrile butadiene styrene (ABS), polypropylene (PP), polyamides, polycarbonate (PC), polylactic acid (PLA) and polyetherimides such as ULTEM®. Parts with overhanging features are supported by the same material as the build, and must be manually removed. Higher precision 
industrial machines often have supports printed in a secondary soluble material. For more details of the FDM process see chapter 3. The first of these examples was published in June 2012, whereby a Fab@Home FDM type extrusion system was used to manufacture a centimetre-scale acetoxysilicone polymer (LOCTITE bathroom sealant) reagent silo. ${ }^{118}$ The Fab@Home printer was developed as part of a project run though Cornell University between the years 2006 and 2012. The project was developed with the aim of developing a low cost, versatile and most importantly customisable printer, allowing open source software and hardware to be shared amongst the wider printing community. The silo was used to store and deliver reagents into a sealed reaction chamber whilst maintaining the structural integrity of the reactor. The reactor was used to undergo a range of inorganic cluster formations along with an organic heterocyclic ring formation. This study successfully demonstrated the applicability of extrusion-based AM procedures towards the manufacture of customisable milli-scale and macro-scale reactors. Whilst comparatively cheap, the type of extrusion process used was crude, typically intended for less demanding prototyping applications. This type of acetoxysilicone polymer is also highly susceptible to chemical attack in a wide range of organic solvents and reagents, therefore limiting its applicability to flow chemistry applications.

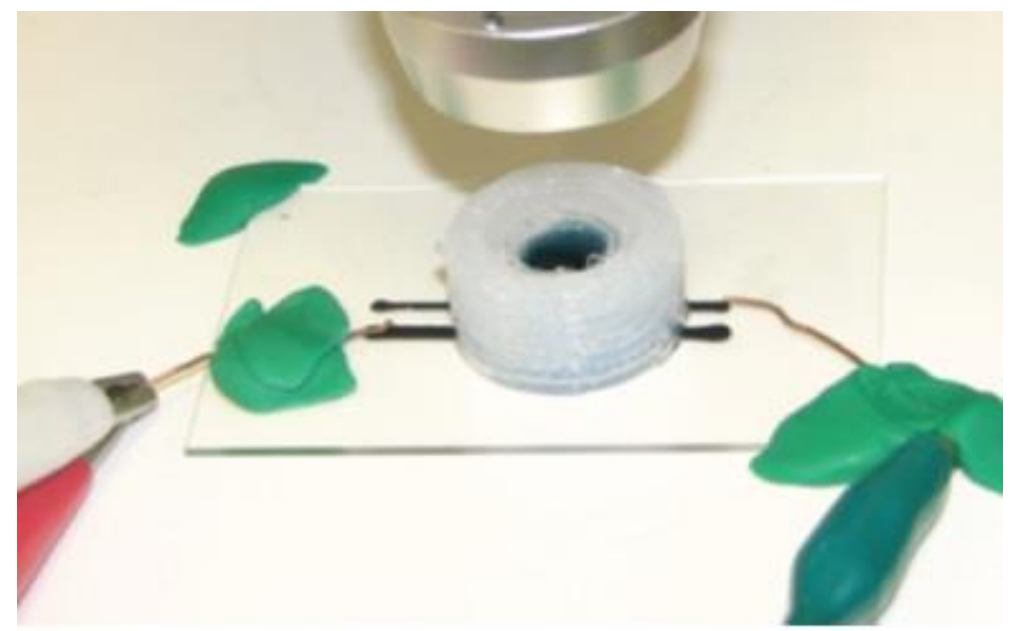

Figure 27: Acetoxysilicone polymer "reactionware" device produced by the University of Glasgow. ${ }^{118}$

Further research was published whereby the group produced three millilitre scale flow devices, using the Bits from Bytes 3DTouch ${ }^{\mathrm{TM}}$ 3D printer to manufacture three polypropylene devices. ${ }^{119,120}$ The three devices all had $0.8 \mathrm{~mm}$ channel diameters and ranged in volume from $60-270 \mu \mathrm{L}$. The parts were the first produced to mimic, on a very small scale, the type of continuous flow channels seen in commercial microfluidic devices. The group 
utilised the parts to perform a range of reactions in flow including an imine formation, an alkylation reaction, and a range of inorganic self-assembly reactions. Polypropylene (PP) is a material with a relatively low melting point $\left(\sim 130^{\circ} \mathrm{C}\right)$ and even lower glass transition temperature, and is therefore not ideally suited to heated flow processes. It is however relatively stable across a wide range of organic solvents such as acetone, methanol and ethyl acetate making it an applicable material for continuous flow processing.

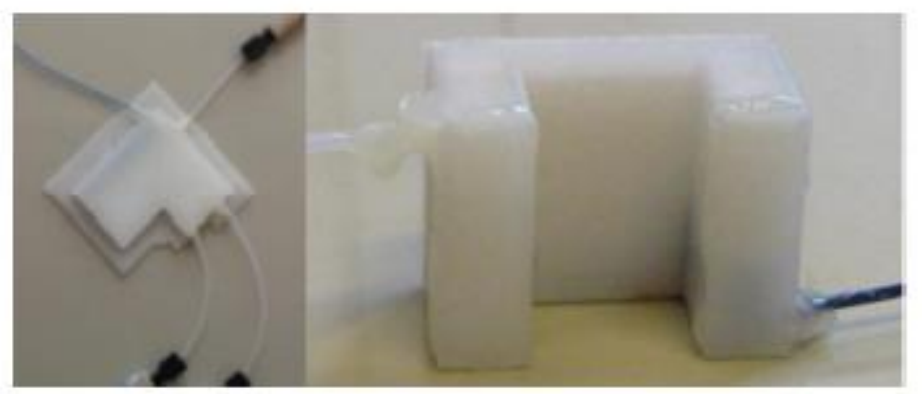

Figure 28: Examples of acetoxysilicone polymer microfluidic devices manufactured via FDM. ${ }^{119}$

The following year the group produced another PP flow device manufactured using the Bits from Bytes 3DTouch ${ }^{\mathrm{TM}}$ 3D printer. ${ }^{121}$ The device had a $1.5 \mathrm{~mm}$ channel diameter and reaction volume of $0.57 \mathrm{~mL}$. The part was attached to a commercially available electrospray ionisation mass spectrometer (ESI-MS) and used to analyse the products of an inorganic supramolecular co-ordination reaction. Using a commercially available system design platform (LabVIEW), they were able to control the flow of the pumping system to mirror the outputs of the ESI-MS. This enabled them to optimise the stoichiometry of the reaction in a real-time on-line analysis.

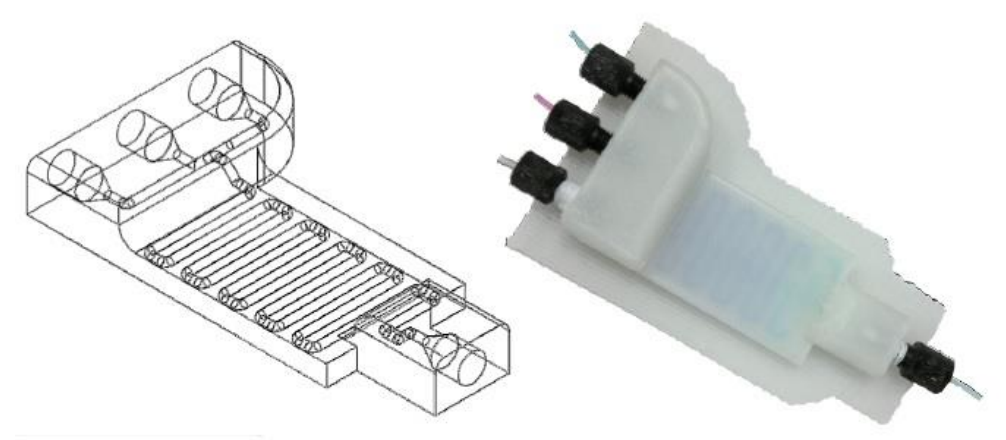

Figure 29: PP flow device used as part of an ESI-MS system. ${ }^{121}$

Later that year the group produced two PP flow channels using the Bits from Bytes 3DTouch ${ }^{\mathrm{TM}}$ 3D printer. ${ }^{122}$ The parts had internal channel diameters of $1.5 \mathrm{~mm}$ making reaction volumes of 0.4 and $0.35 \mathrm{~mm}$ respectively. They connected the reactor outlet to a 
commercially available ATR-FTIR flow cell, then used the devices to perform a range of amine and imine formations, whilst confirming the functional group interconversion online via the use of the FTIR.

A month later (Jun 2013) the group attempted to make liquid and solid handling devices, using PP and the Bits from Bytes 3DTouch ${ }^{\mathrm{TM}}$ 3D printer to produce a serious of interconnecting reaction chambers. ${ }^{123}$ The reaction reagents, catalysts, and purification apparatus were integrated into a single monolithic device. This allowed a multi-step reaction sequence to be performed by simply rotating the device so that the reaction mixture could flow through successive environments under gravity. All this was achieved without the need for any pumps or liquid-handling prior to product retrieval from the reactionware. The group performed a three step reaction which was confirmed via ${ }^{1} \mathrm{H}$ NMR spectroscopy.
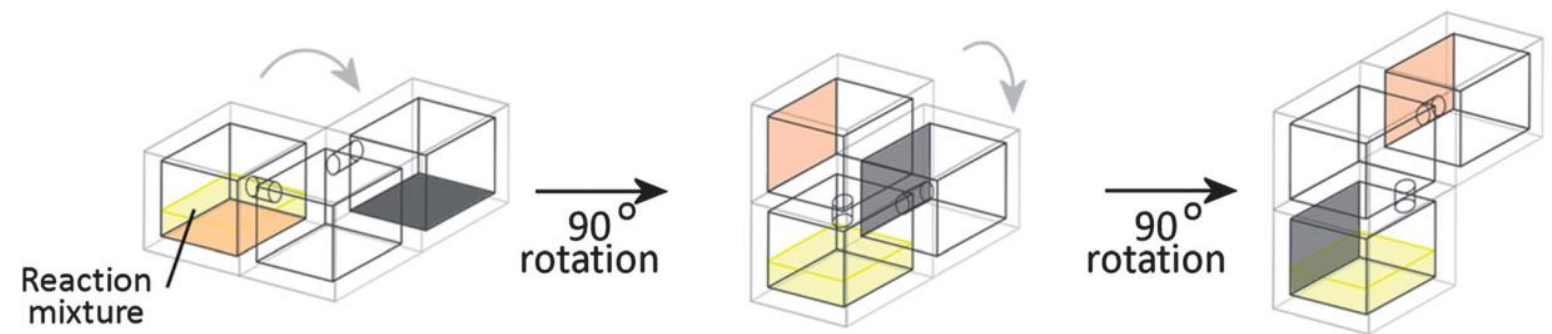

Figure 30: Schematic diagram of the pump-less liquid handling devices used by the Cronin group. ${ }^{123}$

Finally, the group (Jun 2014) published research whereby they used a FDM type Bits from Bytes 3DTouch ${ }^{\mathrm{TM}} 3 \mathrm{D}$ printer, to manufacture a proton exchange membrane (PEM) electrolyser from PP. ${ }^{124}$ A 2-dimensional continuous semi-circular channel was printed to mimic the flow plates of a commercially available PEM. The flow plates were coated with silver paint via a number of discrete surface modifications, and integrated with commercial PEM parts to complete the design. The group then went on to demonstrate the operation of the PEM by testing the performance of the device, quantifying overall efficiency, internal resistances and current-voltage response, whilst cross referencing this data with existing off the shelf products. Although the group did not attempt to use the device for flow processes, it demonstrated the potential of FDM for the manufacture of such devices. The research conducted by this group has certainly demonstrated the flexibility of FDM as a manufacturing process for milli-fluidic devices. Although the range of materials available are not ideal both in terms of chemical compatibility as well as thermal conductivity, FDM is without doubt a relatively inexpensive, user friendly, customisable, and time efficient method of producing functional chemical reactors. 
Research out of Michigan State University has seen mesofluidic devices printed using a commercially available Objet Connex 350 printer and Vero White Plus material. ${ }^{125}$ The material jetting process consists of fine droplets of liquid photopolymer being jetted and cured in situ. Layers of polymer are accumulated on the build plate to create a 3D model. Where supporting material is required for overhanging features, the printer jets a removable support material. The supports can often be removed by hand, or with a solvent mixture. The Vero White material is an acrylate based polymer which makes its susceptible to chemical attack from a range of organic solvents and reagents. It is however very suitable when performing aqueous based biochemical applications such as the ones described as following. The device the group created was a reusable, high throughput fluidic device that incorporated a membrane above a channel in order to study drug transport and affect cells. The device contained 8 parallel channels, $3 \mathrm{~mm}$ wide by $1.5 \mathrm{~mm}$ deep, connected to standard pumping equipment. The part was printed to allow commercially available membranes to be inserted. The surface of these membranes was constructed of a porous polycarbonate membrane, allowing molecular transport to occur from the fluid stream to the above well. The group utilised the part to demonstrate that various antibiotics, when pumped through the cell, would migrate through the membrane wall. They then cultured mammalian cells onto the membrane, before pumping a range of reagents through the device to monitor their interaction with the cells. The study demonstrated the viability of 3D printing as a mode of producing fluidic devices capable of studying drug transport and cell viability in a parallel manner. Devices such as these could easily be optimised to accommodate more high throughput handling systems and plate readers, allowing larger scale industrial applications. 

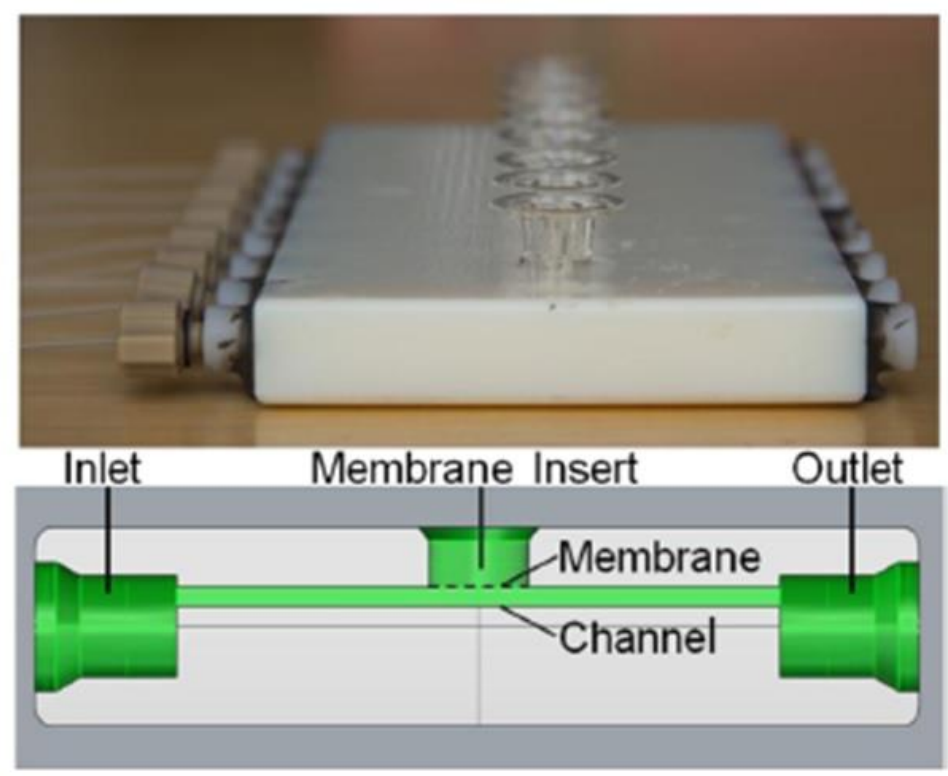

Figure 31: Schematic diagram of the flow cell produced at Michigan State University with integrated membrane inserts. ${ }^{125}$

The same group has also published research whereby the same Objet Connex 350 printer was used to manufacture two 3D printed devices that were used for electrochemical detection. ${ }^{126}$ In both cases the devices were printed in a modular fashion, whereby the electrodes could be easily removed and replaced via a threaded receiving port printed onto the device. The first device represented a $0.5 \mathrm{~mm}$ square microfluidic platform, with ports for integration with glassy carbon or platinum black electrodes. These electrodes were used for dopamine and nitric oxide detection. The second device allowed collection of the biologically relevant analyte adenosine triphosphate (ATP), whilst simultaneously measuring the reduction in oxygen concentration associated with the ATP release stimulus. The study showed that a range of different electrode materials could be integrated into 3D printed devices for a wide range of applications. The modular approach also allows the user to manufacture a complex multicomponent device, identify any design problems, modify the designed part, and reprint quickly and cost effectively for continued study. 


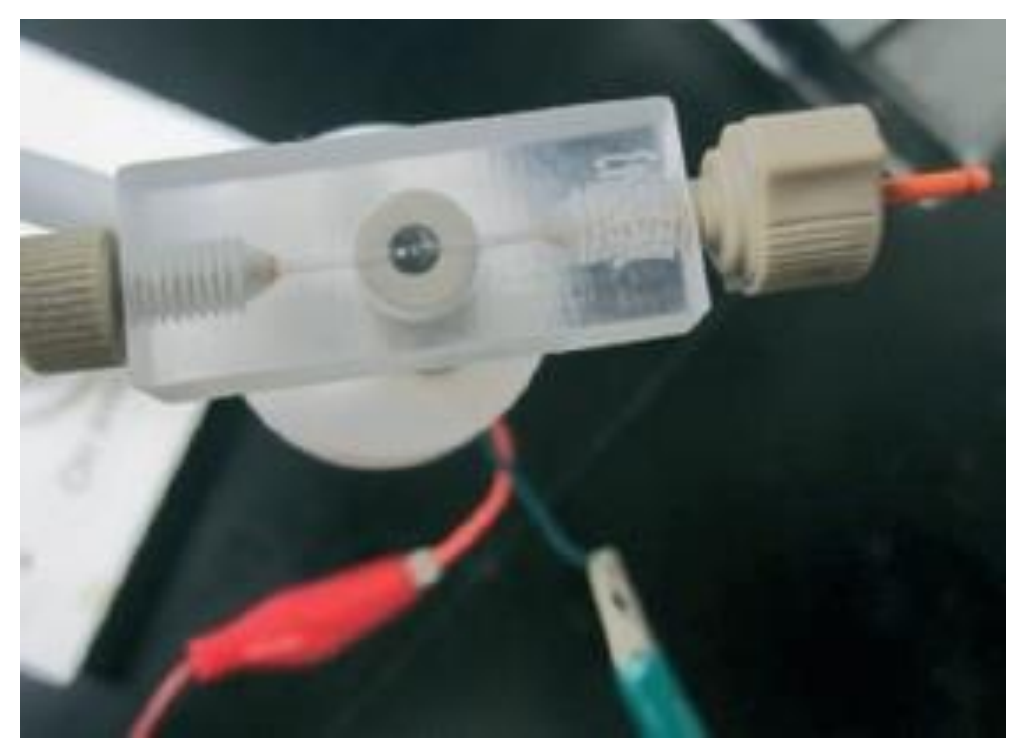

Figure 32: Electrochemical flow cell manufactured using an Objet Connex. ${ }^{127}$

A number of research groups have also published work using 3D printing techniques to fabricate a range of novel microfluidic devices. Malmstadt et al. published research in which they produced a library of compartmentalised microfluidic component and connectors. ${ }^{128}$ These parts were manufactured via stereolithography (SL) from a commercially available Somos WaterShed XC 11122 photoresin. ${ }^{120}$ The SL process uses layer-by-layer photopolymerisation of a liquid resin to create solid three-dimensional parts. SL material choices are typically limited to a narrow range of UV-curable photo-polymers, typically based on acrylates, epoxides and urethanes. Support structures are made from the same material as the build and need to be removed manually, however the uncured resin remaining within the part is easily cleaned away with an alcohol wash. For more details of the SL process see chapter 5 . The type of modular approach to microfluidics used by this group allows the user to build large-scale systems from individual components, designed specifically to meet the demands of the individual microfluidic process. This could potentially reduce the need for expensive pieces of microfluidic apparatus for each individual application. Lee et al. have also published work whereby a Projet HD was used to manufacture a range of microfluidic channel networks. The group used multi-jet modelling (MJM) to manufacture the parts; MJM is an additive manufacturing process which using an industrial print head to jet material onto a build plate in a layer-by-layer process. The print heads jet build and support material simultaneously, allowing a 3D construct to be realised. These materials are similar to those utilised in SL, whereby they require UV light to cure them into a solid part. The support structures used in MJM are often printed in a separate material to the part, usually out of a waxy material which can simply be melted away. This microfluidic construct was applied to 
an immunoreaction as an example of liver cancer diagnosis. Similarly deMello et al. have published research whereby the same Projet HD MJM printer was used to produce a microcapillary device. ${ }^{129}$ This time, the channel network was manufactured via extrusion of a series of glass capillaries, which were held into placed by a 3D printed alignment device. This allowed visualisation of the reagent flow stream via attachment of the part to a standard microscope stage. Folch et al. have also investigated the use of SL for the production of microfluidic devices. ${ }^{130}$ The group manufactured microfluidic devices from DSM Somos® WaterShed XC 11122 resin using a 3D Systems Viper SL system. ${ }^{131}$ The group used the parts to compare a range of factors including build quality, design limitations and optical clarity of the parts when compared to microfluidic devices produced via soft lithography. Other research by Haubelt et al. has seen 3D printing used as a manufacturing aid for conventional ceramic injection moulding processes. ${ }^{132}$ The group used SL to manufacture an epoxy master mould, which was filled with silicone and hardened to produce a negative of the final microfluidic device. By using the SL process the group were able to produce a range of different microfluidic devices, whilst being able to modify and re-design each iteration of the device, in a cost effective and timely manner.

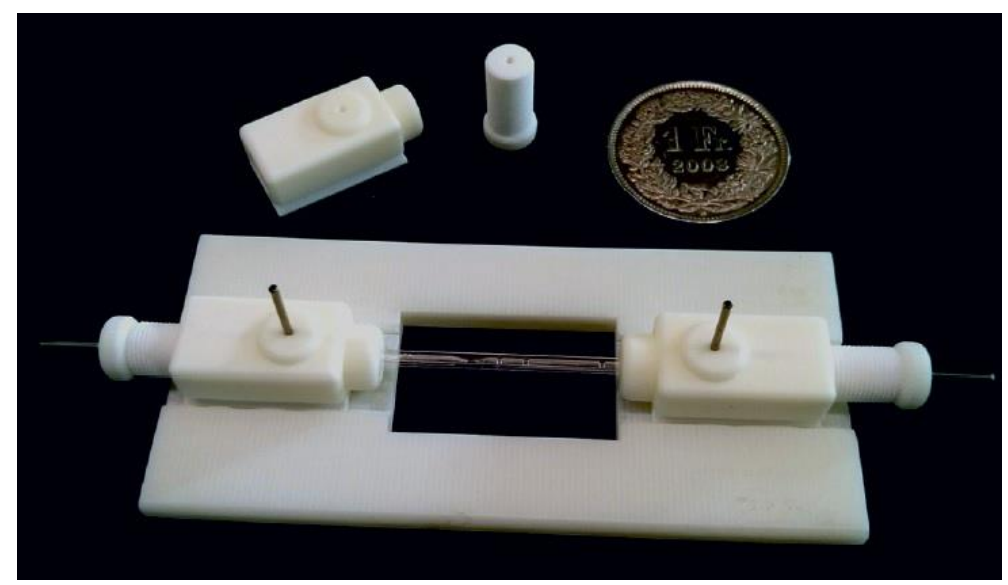

Figure 33: Microcapillary network manufactured via multi-jet modelling. ${ }^{133}$ 


\subsection{Summary}

Despite the field of microfluidic design and manufacture being very well established over a number of decades, there are still clear limitations in terms of production time and cost, manufacturing complexity and availability. AM has the potential to solve all of these issues, however the lack of fundamental research into any AM process for this application has meant that as of yet this method of microfluidic device manufacture remains mostly unexplored. Consequently, we set about exploring the possibility of using a range of AM techniques (SL, SLM, SLS, FDM, UAM) to manufacture these fluidic devices, with the aim of highlighting some of the benefits of using each process, as well as looking to discover novel ways of manufacturing fluidic devices that had functionality in-line with and beyond those of existing microfluidic devices. At the commencement of this research all of these manufacturing processes had never been used for this application, making this area of research a highly exciting and novel field of research. This research also remains to date the only examples of SLM, SLS and UAM flow devices which had been used for flow chemistry applications. 


\section{$\underline{\text { Results and Discussion }}$}

\section{3. $\quad$ Fused Deposition Modelling}

\subsection{Introduction}

Fused deposition modelling (FDM), developed by Stratasys in 1990, is the most widely used inexpensive and commercially available AM process. FDM uses a temperature controlled extrusion nozzle to deposit a viscous molten thermoplastic polymer or wax bead into a threedimensional structure. This process can be used with a range of commercially available thermoplastic polymers, and has been utilised to produce a number of fluidic reaction devices. ${ }^{123}$ Resistive heaters within the nozzle maintain the temperature of the polymer at a temperature just above its melting point, as the filament is fed through the nozzle by a motorised wheel. The material is usually supplied in the form of a long filament, but some machines utilise plastic pellets dispensed through a hopper. ${ }^{134}$ The plastic hardens on contact with either the build plate or the previously deposited layer. Once a single layer has been built, the build platform lowers by a pre-determined distance, and the nozzle extrudes a new layer of polymer. The layer thickness and vertical dimensional accuracy are controlled by an extruder die with a variable diameter. Commercially, reels of thermoplastics such as acrylonitrile butadiene styrene (ABS), polypropylene (PP), polyamides, polycarbonate (PC), polylactic acid (PLA) and polyetherimides such as ULTEM $®$ are available. Generally, FDM materials are limited to these low melting point amorphous thermoplastics, however more chemically stable fluoropolymers such as polyvinylidene fluoride (PVDF) and fluorinated ethylene-propylene (FEP) are theoretically processable.

The accuracy and resolution of the FDM process is limited by the bead size being deposited, which also dictates the layer thickness. Inter-layer bonding depends on localised re-melting of the previous layer by the deposited layer and consequently it is typical for FDM parts to have poorer physical properties perpendicular to the build plane. On small parts, accuracies of around $+/-0.3 \mathrm{~mm}$ are achievable in practice. Typically dimensional accuracy in the $\mathrm{xy}$ build plane can be good approaching $+/-0.1 \mathrm{~mm}$ using the most sophisticated machines. However, accuracy in the z plane is a function of layer thickness. Layer thickness can vary from around 0.35 to $0.1 \mathrm{~mm}$ depending on the exact machine specification, and consequently achievable accuracy will be more in the region of $+/-0.25 \mathrm{~mm}$; even when using sophisticated machines. In the following work the layer thickness used throughout was 0.25 
$\mathrm{mm}$, which is comparatively large compared to other professional standard AM processes. FDM machines are mechanically robust and repeatability is generally good. It may be possible to improve part density and build resolution by using a machine with a smaller layer thickness such as the Stratasys $400 \mathrm{mc}$ (layer thicknesses $=0.127 \mathrm{~mm}$ ), as well as altering build parameters such as raster patterns and nozzle diameter. However, AM techniques that demonstrate strong inter-layer bonding and higher build resolution than the bead of polymer extruded in FDM, are more likely to produce high resolution, non-porous parts suitable for high pressure flow chemistry applications. Despite these limitations, FDM is a relatively inexpensive, user friendly, customisable and time efficient method of producing functional chemical reactors.

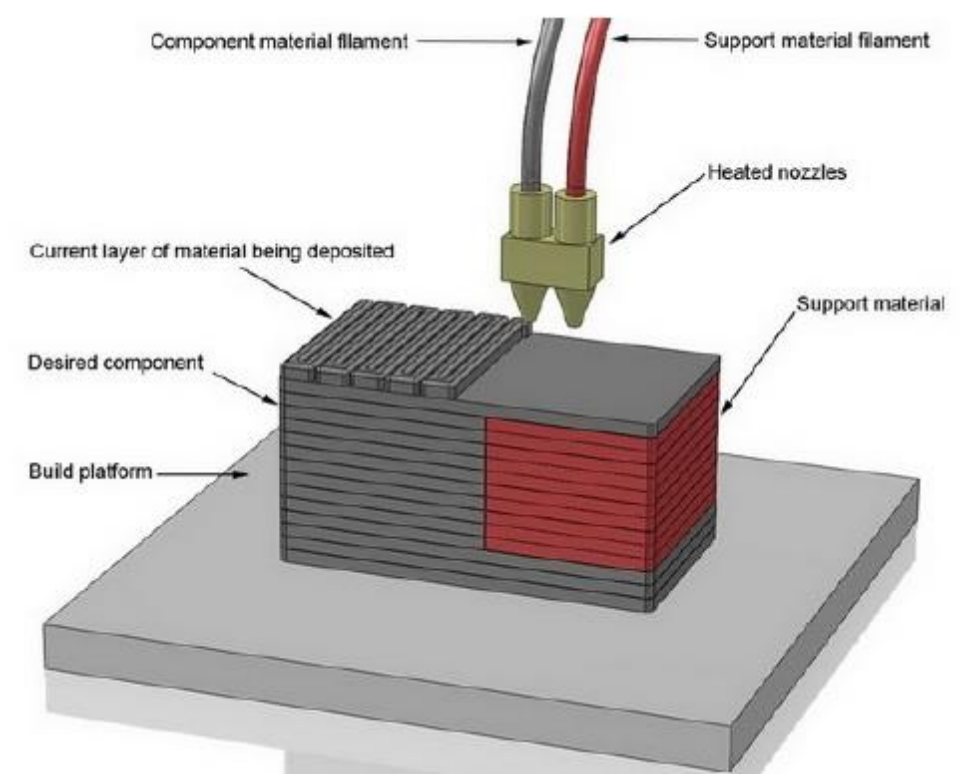

Figure 34: Diagram of the basic FDM build process. ${ }^{134}$

Although FDM is capable of manufacturing parts from a range of thermoplastic polymers, many are not suited to flow chemistry applications due to their poor chemical compatibility. In addition, thermoplastics have poor thermal conductivity, and by definition melt or soften at high temperatures, making some heated flow chemistry applications impractical. ABS is the most commonly applied polymer for FDM. It is an amorphous thermoplastic, which is highly susceptible to swelling/dissolving in organic solvents. Indeed in our own studies, ABS was found to degrade in a wide range of solvents such as THF, dichloromethane, acetone, methanol and toluene. It was found to only be stable in very mild solvents such as isopropanol $(i \mathrm{PrOH})$, water and hexane. As well as having poor chemical compatibility, its relatively low glass transition temperature of $\sim 105^{\circ} \mathrm{C}$ makes it a poor material choice for 
chemical reactor manufacture. Polylactic acid is another commonly used FDM material. Amorphous PLA has a very low glass transition temperature $\left(\sim 65^{\circ} \mathrm{C}\right)$ and would not be suitable for any heated reactions, but is more stable to solvent exposure than ABS, being degraded only in strong solvents such as THF and dichloromethane, and only suffering mild swelling in solvents such as ethyl acetate, methanol and toluene. Figure 35 shows the degradation suffered by PLA cubes after exposure to solvent. We note that the samples show delamination at the interfaces between the individual layers generated during FDM manufacture. It is likely that these weak interfaces and porosity will make FDMmanufactured parts even less solvent resistant than conventionally manufactured parts. PLA has a further disadvantage for chemical applications, in that the ester bond in the backbone is easily broken under many $\mathrm{pH}$ dependent reaction conditions (e.g. acid or base hydrolysis). PC is a more chemically inert amorphous thermoplastic, which has been successfully used to manufacture functional milli-scale flow devices via AM. ${ }^{119} \mathrm{PC}$, although more chemically resistant and thermally stable (glass transition $\sim 147^{\circ} \mathrm{C}$ ) than PLA and ABS, will still degrade slowly in many stronger solvent systems. ULTEM $®$ is an amorphous thermoplastic family of polyetherimides. ULTEM®, whose chemical stability has often been compared to PEEK, has been shown to be stable in many stronger solvents such as toluene and ethyl acetate. Its glass transition temperature of $\sim 216^{\circ} \mathrm{C}$ also makes it more than capable of withstanding the thermal demands of most continuous flow processes. ULTEM ${ }^{\circledR}$ is perhaps the most appropriate commercially available material for use as a continuous flow reactor, however it still does not support the wide range of reaction conditions of a commercial PEEK reactor. Paradoxically, the high glass transition temperature makes it difficult to produce ULTEM® FDM parts using commercially available equipment because of the high extrusion temperature required.

Two FDM machines were used during the course of the research. A PP3DP UP! Printer was used to manufacture ABS parts, whilst a MakerBot Replicator was used to manufacture PLA parts. The UP! Printer, which was used for most of the work, has a build platform of $140 \mathrm{x}$ $140 \times 135 \mathrm{~mm}(\mathrm{x}, \mathrm{y}, \mathrm{z})$. The machine has a number of predefined layer thicknesses ranging from $150-400 \mu \mathrm{m}$ and is capable of processing both ABS and PLA. This machine retails at around $£ 1000$, making it affordable to a wide audience. The MakerBot Replicator has a much larger build platform of $252 \times 199 \times 150 \mathrm{~mm}(\mathrm{x}, \mathrm{y}, \mathrm{z})$. The machine is capable of producing more precise layers of $100 \mu \mathrm{m}$ and is also capable of processing both ABS and PLA, however does retail at around $£ 2500$ making it less accessible than the UP! printer, 


\subsection{Results and Discussion}

During the course of this research all FDM parts were manufactured from either ABS or PLA. The selection of these two materials was purely a matter of availability. The solvent compatibility for these materials was determined by manufacturing $1 \mathrm{~cm}^{3}$ blocks of ABS/PLA which were subjected to a range of solvents. Each cube was weighed before and after exposure to a solvent for the specified time period, with the weight gain/loss through swelling being quantified. Visual observations of the parts were also made throughout. Variations in dimensions due to swelling were also recorded. Initial variations in weights were a reflection of the effectiveness of the support structure removal process, rather than the accuracy of the build. All dimensional measurements were conducted in the $\mathrm{z}$ plane of the build. Slight degradation (SD) of parts was defined as any visible damage to the surface of the part that did not alter the overall shape of the cube. Total degradation (TD) was defined as any visible damage to the surface of the part that did alter the overall shape of the cube. Delamination was defined as swelling that lead to individual layers of the part delaminating from one another (D). Colour changes were a loss of colouring in the part (CC).
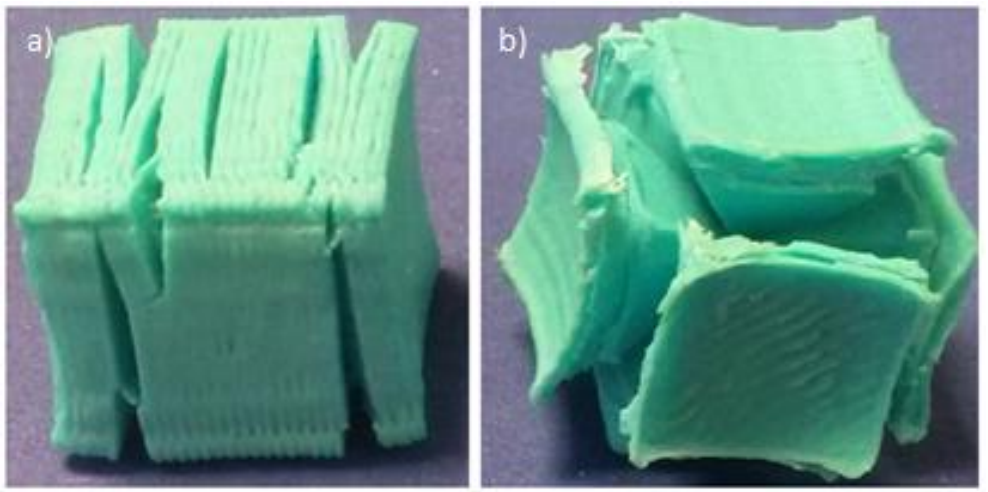

Figure 35: a) delamination of an FDM cube during materials testing b) total degredation (TD) of an FDM cube during materials testing. 
ABS Cubes:

\begin{tabular}{|c|c|c|c|c|c|c|}
\hline Solvent & $\begin{array}{c}\text { Weight } \\
\text { at } \mathbf{t}=\mathbf{0} \mathrm{h} \\
\text { (g) }\end{array}$ & $\begin{array}{c}\text { Weight at } \\
\mathrm{t}=0.5 \mathrm{~h} \\
\text { (g) }\end{array}$ & $\begin{array}{c}\text { Weight } \\
\text { at } t=2 h \\
\text { (g) }\end{array}$ & $\begin{array}{c}\text { Weight at } \\
\mathrm{t}=24 \mathrm{~h} \\
\text { (g) }\end{array}$ & $\begin{array}{c}\text { Weight } \\
\text { change } \\
(\%)\end{array}$ & $\begin{array}{c}\text { Dimensional } \\
\text { change } \\
(+/-\mathbf{m m})\end{array}$ \\
\hline $\begin{array}{l}\text { Ethyl } \\
\text { acetate }\end{array}$ & 0.759 & $\begin{array}{l}0.862 \\
(\mathrm{SD})\end{array}$ & $\mathrm{TD}$ & $\mathrm{TD}$ & N/A & N/A \\
\hline $\begin{array}{l}\text { Diethyl } \\
\text { ether }\end{array}$ & 0.753 & $\begin{array}{l}0.872 \\
(\mathrm{CC})\end{array}$ & 1.018 & 1.115 & 48.07 & 0.75 \\
\hline Toluene & 0.762 & $\begin{array}{l}0.866 \\
(\mathrm{SD})\end{array}$ & TD & TD & N/A & N/A \\
\hline $\mathrm{CH}_{2} \mathrm{Cl}_{2}$ & 0.765 & TD & TD & $\mathrm{TD}$ & N/A & N/A \\
\hline THF & 0.755 & TD & TD & TD & N/A & N/A \\
\hline $\begin{array}{c}\text { Light } \\
\text { petroleum }\end{array}$ & 0.762 & 0.762 & 0.775 & 0.771 & 1.18 & 0.02 \\
\hline $\mathrm{MeOH}$ & 0.759 & 0.776 & 0.802 & 0.890 & 17.26 & 0.59 \\
\hline$i \mathrm{PrOH}$ & 0.754 & 0.751 & 0.775 & 0.782 & 3.71 & 0.02 \\
\hline Hexane & 0.765 & 0.767 & 0.768 & 0.776 & 1.44 & 0.01 \\
\hline Acetone & 0.754 & TD & TD & $\mathrm{TD}$ & N/A & N/A \\
\hline
\end{tabular}

Table 1: Solvent compatibility data for AM ABS cubes. 
PLA Cubes:

\begin{tabular}{|c|c|c|c|c|c|c|}
\hline Solvent & $\begin{array}{c}\text { Weight } \\
\text { at } \mathrm{t}=0 \mathrm{~h} \\
\text { (g) }\end{array}$ & $\begin{array}{c}\text { Weight at } \\
\mathrm{t}=0.5 \mathrm{~h} \\
\text { (g) }\end{array}$ & $\begin{array}{c}\text { Weight } \\
\text { at } t=2 \mathrm{~h} \\
\text { (g) }\end{array}$ & $\begin{array}{c}\text { Weight at } \\
\mathbf{t}=\mathbf{2 4} \mathrm{h} \\
\text { (g) }\end{array}$ & $\begin{array}{c}\text { Weight } \\
\text { change } \\
(\%)\end{array}$ & $\begin{array}{c}\text { Dimensional } \\
\text { change } \\
(+/-\mathbf{m m})\end{array}$ \\
\hline $\begin{array}{c}\text { Ethyl } \\
\text { acetate }\end{array}$ & 1.197 & 1.288 & 1.298 & 1.603 (D) & 33.92 & N/A \\
\hline $\begin{array}{l}\text { Diethyl } \\
\text { ether }\end{array}$ & 1.209 & 1.245 & 1.254 & 1.348 & 11.50 & 0.61 \\
\hline Toluene & 1.208 & 1.290 & 1.291 & 1.523 (D) & 26.71 & N/A \\
\hline $\mathrm{CH}_{2} \mathrm{Cl}_{2}$ & 1.202 & TD & TD & TD & N/A & N/A \\
\hline THF & 1.209 & 1.327 & 1.338 & $\mathrm{D}$ & N/A & N/A \\
\hline $\begin{array}{c}\text { Light } \\
\text { petroleum }\end{array}$ & 1.202 & 1.203 & 1.203 & 1.198 & 0.33 & 0.12 \\
\hline $\mathrm{MeOH}$ & 1.198 & 1.217 & 1.217 & 1.228 & 2.50 & 0.20 \\
\hline$i \mathrm{PrOH}$ & 1.200 & 1.209 & 1.206 & 1.210 & 0.83 & 0.00 \\
\hline Hexane & 1.202 & 1.206 & 1.206 & 1.206 & 0.33 & 0.00 \\
\hline Acetone & 1.201 & 1.285 & 1.302 & $\mathrm{D}$ & N/A & N/A \\
\hline
\end{tabular}

Table 2: Solvent compatibility data for AM PLA cubes.

Given that both materials available to us degraded significantly in a wide range of organic solvents, the majority of work using FDM was done as an inexpensive method of prototyping builds for other more costly processes such as SL and SLM.

\subsubsection{FDM Parts 1-4}

\section{Design, build and post processing:}

Before the final design for SL part 10 (Chapter 7.2.2) was completed, the design was prototyped in PLA using an UP! Plus 3D Printer. Although it was possible to accurately measure the external features of the Agilent flow cell that the design would be based on, due to constraints with the SL and SLM build processes it was not possible to directly mimic the design of the part. This meant that the internal dimensions of the diode array detector (DAD) compartment of an Agilent HPLC must be determined, so that the geometrical constraints that were being worked within were known. Unfortunately, the lack of accessibility to this 
compartment made precise measurements difficult. Therefore prototyping the design using a less expensive manufacturing process than SL was necessary to optimise the part in a costefficient manner. Five different designs were produced, making subtle variations relating to the length and depth of the part, as well as design features such as the position of the sprung clip and the reagent inlets and outlets. The final optimised design was used as the basic model which SL part 10 and SLM part 9 were based around. The four parts were built with the dimensions outlined in Table 3.

\begin{tabular}{|c|c|c|c|c|}
\hline Part & Length (mm) & Width $(\mathbf{m m})$ & Height (mm) & Volume $\left.\mathbf{( c m}^{\mathbf{3}}\right)$ \\
\hline 1 & 113.90 & 26.40 & 50.00 & 31.18 \\
\hline 2 & 113.90 & 26.40 & 47.10 & 32.66 \\
\hline 3 & 113.90 & 26.40 & 40.60 & 39.86 \\
\hline 4 & 86.20 & 26.40 & 32.50 & 31.18 \\
\hline
\end{tabular}

Table 3: FDM Parts 1-4 Build Dimensions.

\section{Results/Discussion:}

Measurements of the four parts were taken in the $\mathrm{x}$ and $\mathrm{y}$ planes, comparing this data with the original CAD to determine dimensional accuracy.

\begin{tabular}{|c|c|c|c|c|c|}
\hline Part & Dimension & $\begin{array}{c}\text { Actual } \\
\text { measurement } \\
(\mathbf{m m})\end{array}$ & $\begin{array}{c}\text { CAD } \\
\text { measurement } \\
(\mathbf{m m})\end{array}$ & $\begin{array}{c}\text { Difference } \\
(+/-\mathbf{m m})\end{array}$ & $\begin{array}{c}\text { Variation } \\
(\%)\end{array}$ \\
\hline 1 & $\mathrm{X}$ & 113.86 & 113.90 & 0.04 & 0.04 \\
\hline 2 & $\mathrm{X}$ & 113.84 & 113.90 & 0.06 & 0.05 \\
\hline 3 & $\mathrm{X}$ & 113.80 & 113.90 & 0.10 & 0.09 \\
\hline 4 & $\mathrm{X}$ & 86.09 & 86.20 & 0.11 & 0.13 \\
\hline 1 & $\mathrm{Y}$ & 26.55 & 26.40 & 0.15 & 0.41 \\
\hline 2 & $\mathrm{Y}$ & 26.57 & 26.40 & 0.17 & 0.64 \\
\hline 3 & $\mathrm{Y}$ & 26.50 & 26.40 & 0.10 & 0.37 \\
\hline 4 & $\mathrm{Y}$ & 26.51 & 26.40 & 0.11 & 0.41 \\
\hline
\end{tabular}

Table 4: Optical Calliper Measurements of FDM Parts 1-4.

A discussion about the accuracy and repeatability of parts built via the FDM process will follow later in the chapter (3.2.2). 


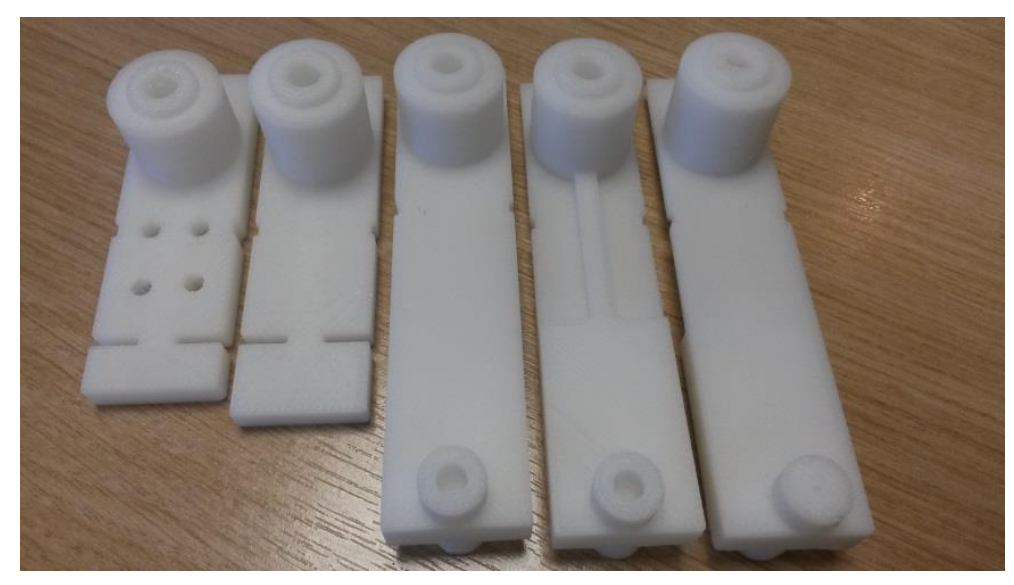

Figure 36: FDM Parts 1-4.

\subsubsection{FDM Parts 5-8}

Some work was also conducted whereby FDM was used as a method of embedding functional components into fluidic devices. This was to be achieved by segmenting the designs into multiple sections, whereby after each section had been built an object could be embedded into a predefined cavity within the part. Having inserted the functional component, it would then be possible to define a new build plate as the upper surface of the previous build. The next section of the part would be built directly on top of the previous part, hopefully leading to the creation of a single body.

\section{FDM Part 5:}

\section{Design, build and post processing:}

An initial idea was hypothesised, whereby a $\mathrm{NaCl}$ disk could be embedded across a flow channel, allowing the part to be used as a spectroscopic flow cell. FDM part 5 was designed as three sections, which would allow $2 \mathrm{NaCl}$ disks $(25 \mathrm{~mm}$ diameter) to be inserted either side of a $3 \mathrm{~mm}$ flow channel. These disks would intersect another $3 \mathrm{~mm}$ channel running perpendicular to the flow channel, which would act as a spectroscopic viewing window. The part was manufactured from ABS using an UP! Plus 3D Printer, and the three sections were built with the dimensions outlined in Table 5 . 


\begin{tabular}{|c|c|c|c|c|}
\hline Section & Length $(\mathbf{m m})$ & Width $(\mathbf{m m})$ & Height $(\mathbf{m m})$ & Volume $\left.\mathbf{( c m}^{\mathbf{3}}\right)$ \\
\hline A & 60 & 35 & 24 & 48.3 \\
\hline B & 60 & 35 & 21 & 41.6 \\
\hline C & 60 & 35 & 20 & 41.9 \\
\hline
\end{tabular}

Table 5: FDM Part 5 Build Dimensions.

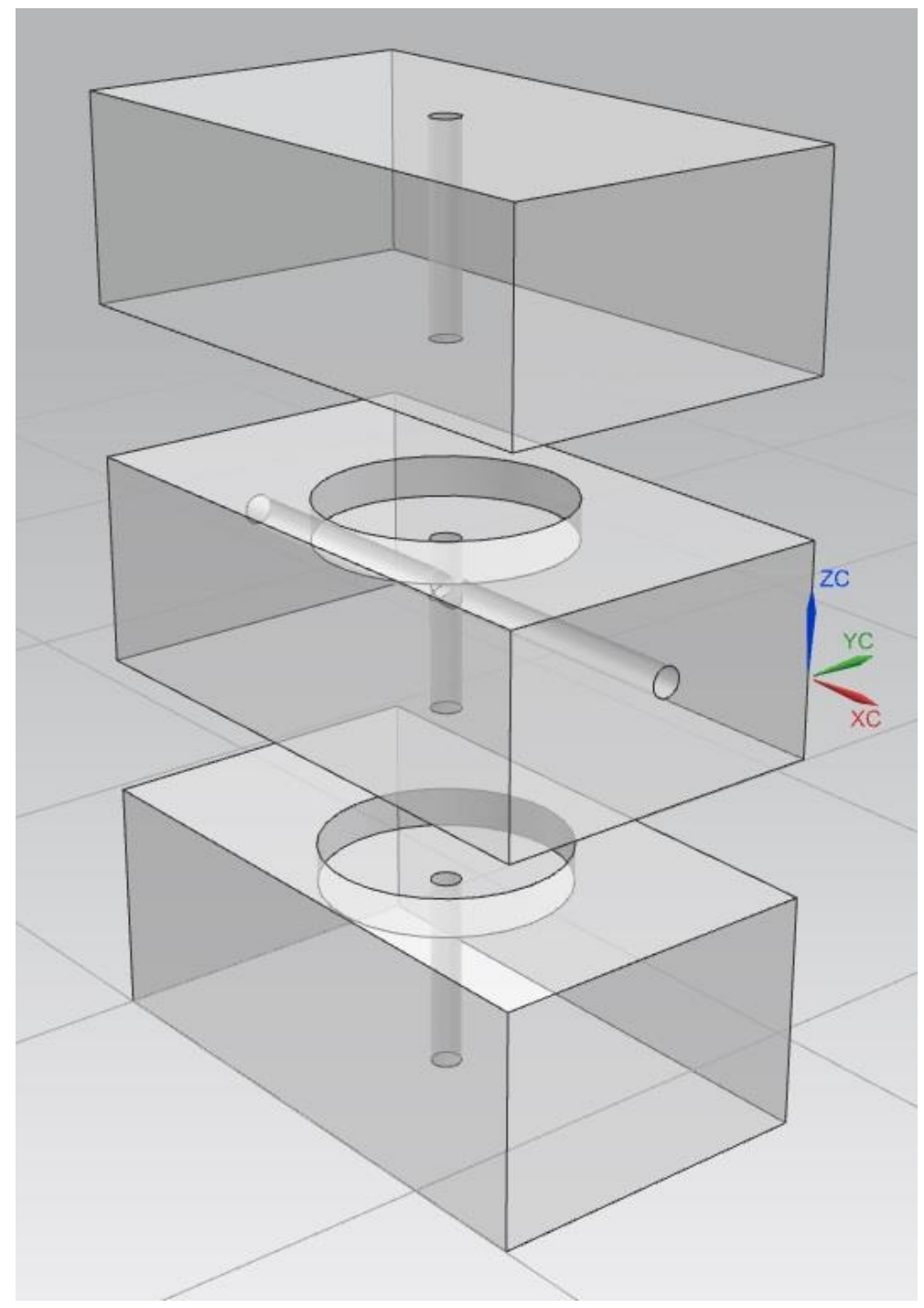

Figure 37: CAD Drawings of FDM Part 5.

\section{Results/Discussion:}

Despite a number of attempts to manufacture FDM part 5, the three sections of the build would invariably fail to properly adhere to one another. The main issue was delamination of the part across the interface between the $\mathrm{NaCl}$ disk and the $\mathrm{ABS}$ layer adhering to it. Often this would transcend itself as the bead of molten plastic failing to adhere to the disc, causing 
it to be dragged around the build platform, leading to total build failure. It was possible this was being caused by the size of the object that we were trying to embed, or by a fundamental problem with bonding $\mathrm{ABS}$ to a $\mathrm{NaCl}$ surface.

\section{FDM Part 6:}

\section{Design, build and post processing:}

After the failure of the previous build, it was hypothesised that we may be able facilitate bonding between the three build sections by reducing the size of the object being embedded. This would hopefully limit the delamination of layers that was seen previously. A glass wafer $(18 \mathrm{~mm} \times 18 \mathrm{~mm}$ ) was proposed as a smaller (and less expensive) alternative to the $\mathrm{NaCl}$ disks. The part was again manufactured from three sections, which would allow two glass wafers to be inserted either side of a $3 \mathrm{~mm}$ flow channel. These wafers would be placed within a $0.2 \mathrm{~mm}$ cavity so that they would sit flush with the surface of the build. The part was manufactured from ABS using an UP! Plus 3D Printer, and the three sections were built with the dimensions outlined in Table 6.

\begin{tabular}{|c|c|c|c|c|}
\hline Section & Length $(\mathbf{m m})$ & Width $(\mathbf{m m})$ & Height $(\mathbf{m m})$ & Volume $\left.\mathbf{( c m}^{\mathbf{3}}\right)$ \\
\hline A & 28 & 28 & 10 & 7.7 \\
\hline B & 28 & 28 & 5.2 & 3.8 \\
\hline C & 28 & 28 & 10 & 7.8 \\
\hline
\end{tabular}

Table 6: FDM Part 6 Build Dimensions. 


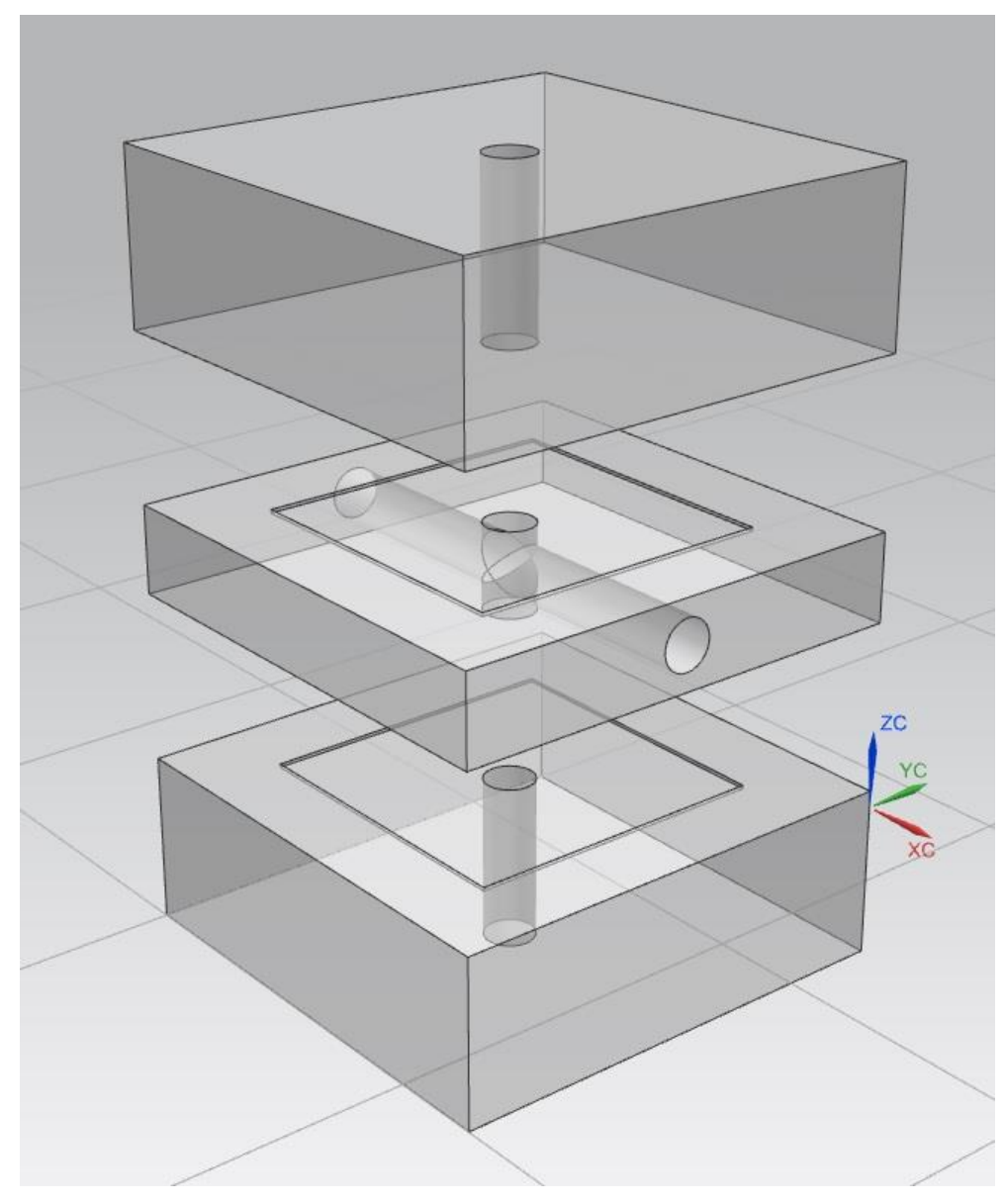

Figure 38: CAD Drawings for FDM Part 6.

\section{Results/Discussion:}

Unfortunately, the design was once again was unsuccessful due to movement of the glass wafers during the build process. As each new layer was extruded over the top of the wafer, the wafer would be moved around the build plate by the motion of the extrusion nozzle. It was hypothesised that this could be counteracted by burying the wafer within a deeper cavity to prevent any movement.

\section{FDM Part 7:}

\section{Design, build and post processing:}

FDM part 7 was again a three section build, whereby two glass wafers would be placed within a much deeper $(5.2 \mathrm{~mm})$ port. These wafers would be held in place during the build process and enclosed within the build by the next section of the design. The part was manufactured from ABS using an UP! Plus 3D Printer, and the three sections were built with the dimensions outlined in Table 7. 


\begin{tabular}{|c|c|c|c|c|}
\hline Section & Length $(\mathbf{m m})$ & Width $(\mathbf{m m})$ & Height $(\mathbf{m m})$ & Volume $\left.\mathbf{( c m}^{\mathbf{3}}\right)$ \\
\hline A & 28 & 28 & 10 & 6.1 \\
\hline B & 28 & 28 & 15 & 7.7 \\
\hline C & 28 & 28 & 7 & 3.1 \\
\hline
\end{tabular}

Table 7: FDM Part 7 Build Dimensions.

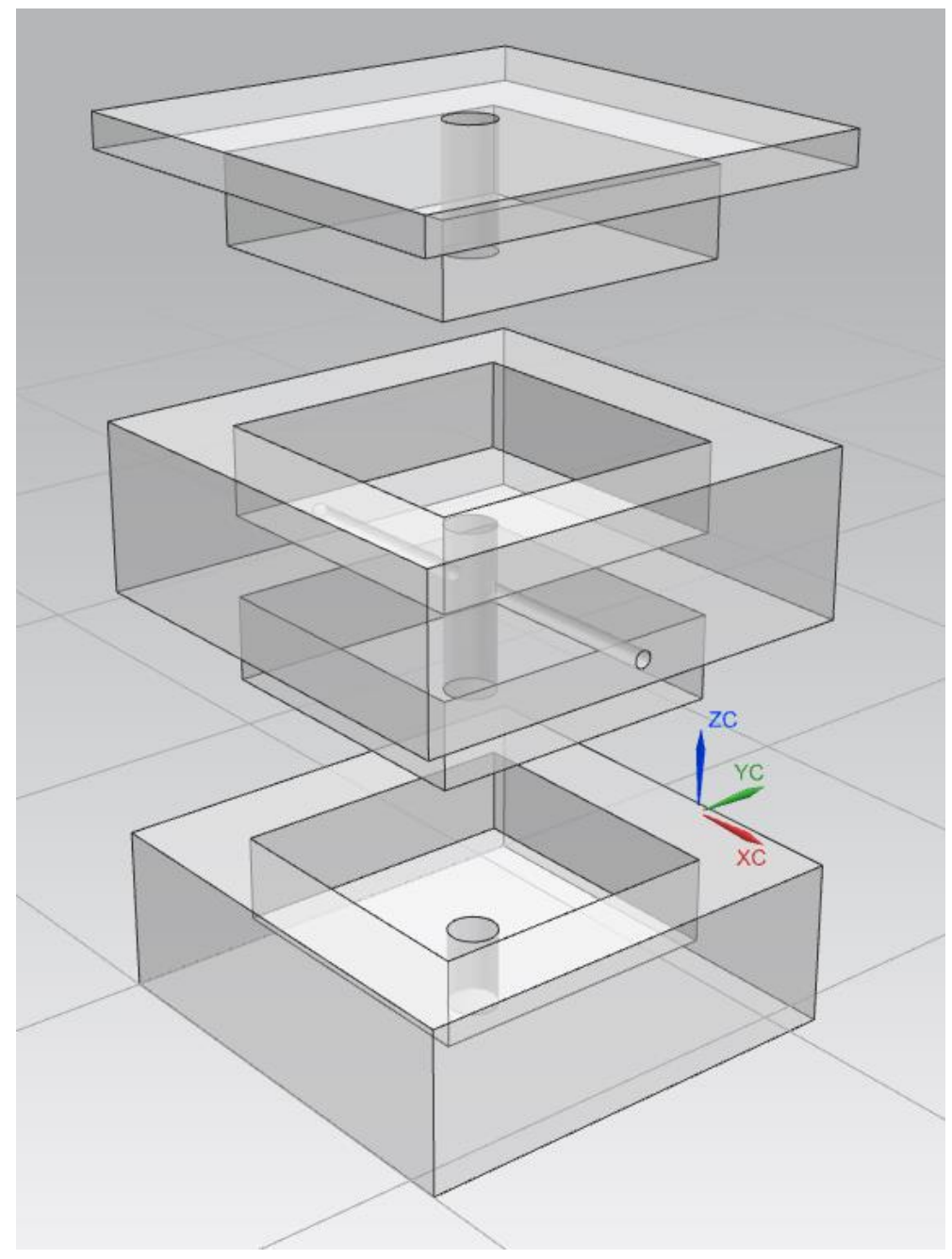

Figure 39: CAD Drawings for FDM Part 7.

\section{Results/Discussion:}

The part built successfully with each of the three sections adhering to form a single solid body. Development of this design to allow standard PEEK LC fittings, as well as SubMiniature Version A (SMA) connectors to be fitted, will allow the part to be connected to suitable pumps and spectroscopic device. This ability to pause and restart builds, whilst inserting discrete elements, is a significant advantage of using FDM over other AM processes, whereby the build cannot be easily stopped. 


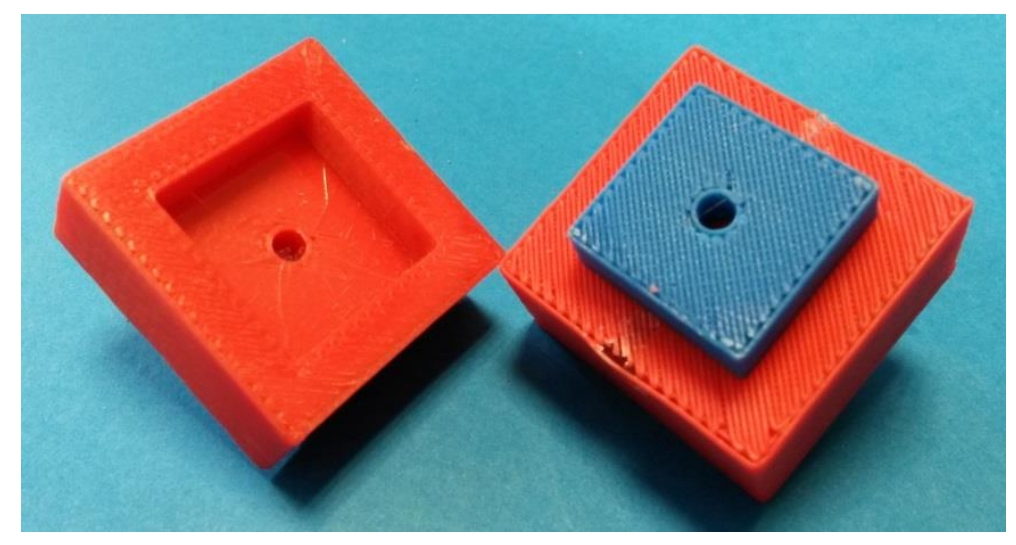

Figure 40: FDM Part 7 built in separate sections to highlight the individual layers.

\section{FDM Part 8:}

Design, build and post processing:

FDM part 8 was again a three section build, based around the design of FDM part 7, allowing the part to be connected to an Ocean Optics S2000 spectrophotometer and a Mikropack DH2000 balanced deuterium/halogen light source. The part was manufactured from ABS using an UP! Plus 3D Printer, and the three sections were built with the dimensions outlined in Table 8.

\begin{tabular}{|c|c|c|c|c|}
\hline Section & Length $(\mathbf{m m})$ & Width $(\mathbf{m m})$ & Height $(\mathbf{m m})$ & Volume $\left.\mathbf{( c m}^{\mathbf{3}}\right)$ \\
\hline A & 28 & 35 & 16 & 6.3 \\
\hline B & 28 & 35 & 17 & 9.3 \\
\hline C & 28 & 35 & 13 & 3.3 \\
\hline
\end{tabular}

Table 8: FDM Part 8 Build Dimensions. 


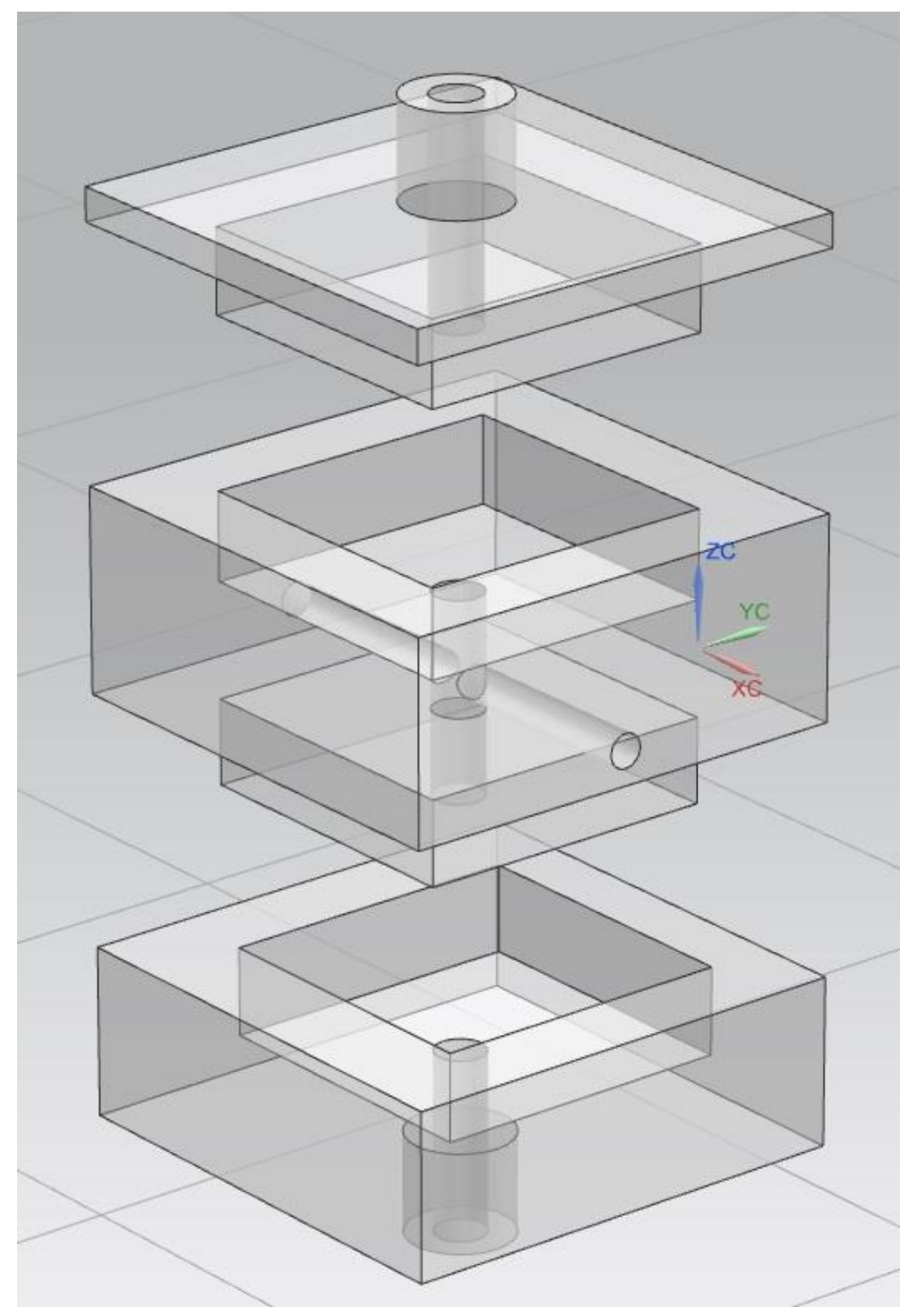

Figure 41: CAD Drawings of FDM Part 8.

The flow inlet and outlets were tapped with a 1/4"-28 UNF thread, however the material proved very brittle and not ideally suited to machining.

\section{Results/Discussion:}

The part built successfully allowing us to connect it to a suitable light source and detector. Unfortunately, when a solution of methylene blue was pumped along the flow channel using a syringe, the part leaked around the glass wafer/part interface. After various attempts to seal the part by altering part geometries, as well as build parameters, the part was unable to be sealed along this interface. Despite these failures there was clearly promise in the area of embedding components into an AM fluidic device, and this work lead to further research embedding quartz windows into SL and SLM parts (Chapter 7.2). 


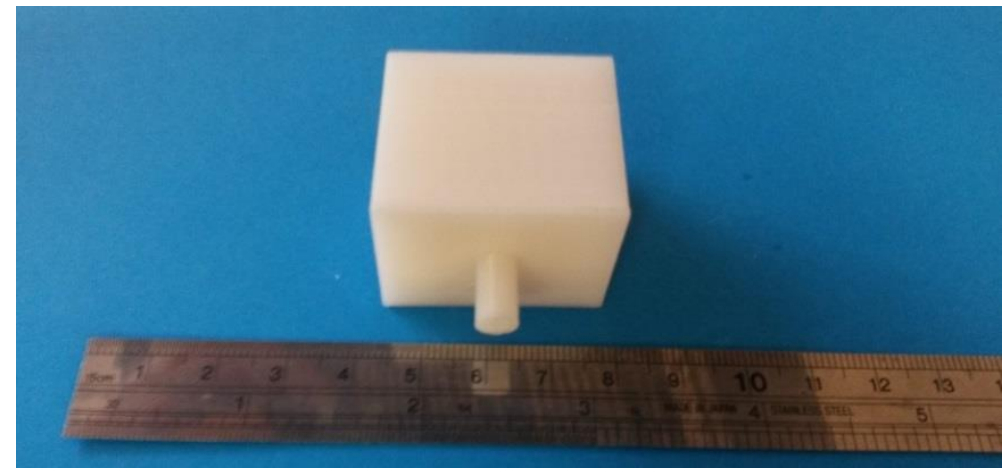

Figure 42: FDM Part 8

Measurements of the parts were taken in the $\mathrm{x}$ and $\mathrm{y}$ plane, comparing this data with the original CAD to determine dimensional accuracy.

\begin{tabular}{|c|c|c|c|c|c|}
\hline Part & Dimension & $\begin{array}{c}\text { Actual } \\
\text { measurement } \\
(\mathbf{m m})\end{array}$ & $\begin{array}{c}\text { CAD } \\
\text { measurement } \\
(\mathbf{m m})\end{array}$ & $\begin{array}{c}\text { Difference } \\
(+/-\mathbf{m m})\end{array}$ & $\begin{array}{c}\text { Variation } \\
(\%)\end{array}$ \\
\hline 5 & $\mathrm{X}$ & 27.87 & 28.00 & 0.13 & 0.47 \\
\hline 6 & $\mathrm{X}$ & 27.80 & 28.00 & 0.20 & 0.72 \\
\hline 7 & $\mathrm{X}$ & 27.81 & 28.00 & 0.19 & 0.68 \\
\hline 8 & $\mathrm{X}$ & 27.85 & 28.00 & 0.25 & 0.54 \\
\hline 5 & $\mathrm{Y}$ & 27.85 & 28.00 & 0.15 & 0.54 \\
\hline 6 & $\mathrm{Y}$ & 28.00 & 28.00 & 0.00 & 0.00 \\
\hline 7 & $\mathrm{Y}$ & 27.94 & 28.00 & 0.06 & 0.21 \\
\hline 8 & $\mathrm{Y}$ & 35.11 & 35.00 & 0.11 & 0.31 \\
\hline
\end{tabular}

Table 9: FDM Part 6 Build Dimensions.

The dimensional accuracy of parts manufactured via the FDM process from PLA was generally very good. The four parts which were built had dimensions which were on average within $105 \mu \mathrm{m}$ of the intended dimensions. This equated to around a $0.27 \%$ discrepancy in geometrical accuracy. Comparatively, the dimensional accuracy of parts manufactured via the FDM process from ABS was slightly lower. The four parts which were built had dimensions which were on average within $136 \mu \mathrm{m}$ of the intended dimensions. This equated to around a $0.43 \%$ discrepancy in geometrical accuracy. Both materials compared favourably to SLM manufacturing, and were only slightly less accurate than the parts produced via SL. Dimensional accuracy could be increased further by switching to a higher specification FDM such as a Stratasys $400 \mathrm{mc}$ printer. 


\subsection{Conclusion}

The lack of suitable material choices limits FDM manufactured parts to use with chemistry performed in mild organic or aqueous solvent systems. Future development of the FDM materials portfolio may make it a very cost efficient method of producing functional flow reactors with complex designs. Fluoropolymers such as PVDF (melting point $177^{\circ} \mathrm{C}$ ) and FEP (melting point $260^{\circ} \mathrm{C}$ ), are stable across a wide range of organic solvents and are theoretically processable within an FDM system. Although these types of materials are not currently commercially available in a heavily consumer driven market, the development of this type of industrial application will hopefully lead to more materials development work in this area.

However, FDM does offer some potential in terms of chemical reactor design and manufacture. The user friendly/customisable nature of the entire design and manufacturing process allows precise control and rapid optimisation of reactor dimensions, geometry and functionality, whilst producing parts at a fraction of the cost of other AM processes. The accessibility of FDM to the general public and the shared knowledge pool consequently being developed, may also open up flow processes to a much wider group of industrial and academic chemists.

\section{4. $\quad$ Future Work}

A focus on more chemically and thermally compatible commercially available FDM materials such as polycarbonate (PC) and ULTEM ${ }^{\circledR}$ will open up FDM manufacture to a much wider range of continuous flow processes. The UP! Printer used during this research is a low cost FDM system which may not be ideally suited to this application, however the recent availability of industrial machines such as the Stratasys $400 \mathrm{mc}$ will hopefully be able to produce parts with physical properties more suited to this application. 


\section{4. $\quad$ Laser Sintering}

Laser sintering (LS), or selective laser sintering (SLS), is a powder-based AM technique that uses a high-powered laser to scan and consolidate powder particles into a solid 3D object. Typical LS machines contain three temperature and atmosphere controlled chambers. This will include a build chamber which contains the "powder bed" or "part bed" where the parts are manufactured. The other chambers are feed chambers, which store the feedstock powder prior to it being swept across the build area by a roller or wiper blade. These chambers have a piston controlled platform which moves incrementally upwards or downwards allowing a fresh layer of powder to be deposited on top of the previously sintered layers. The powder chamber will be pre-heated to just below the melting point of the material being used. A laser is used to selectively sinter the pre-defined geometry, which defines the 3D shape which has been designed, sliced and inputted into the LS software. A number of parameters such as feed and bed temperature, roller speed, laser power, and scan count (the number of times the laser beam traverses a scan vector per layer) can be altered to control the amount of energy inputted into the surface of the material which will ultimately determine the resolution and repeatability of the part being processed. ${ }^{135}$

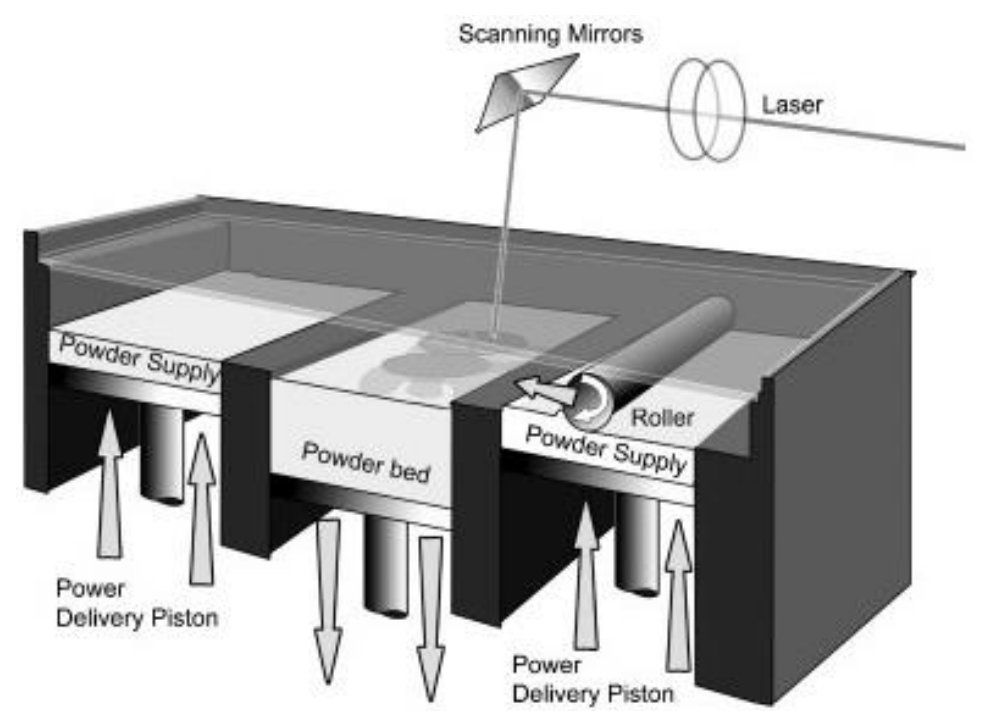

Figure 43: A schematic diagram of the LS process. ${ }^{135}$

LS is capable of building high resolution parts from a wide range of powder materials including polymers, ceramics and metals, however the term LS is usually reserved for polymers. Resolution is limited by a combination of the particle size (typically 50-100 $\mu \mathrm{m}$ ) and the laser spot size. The polymer materials available for LS are suitable to the demands of a flow reactor, with a number of more chemically and thermally compatible materials 
available allowing a more diverse range of chemistry to be performed within the reactor. Support structures are not required as the part being built is supported by the surrounding powder, removing the need for post-processing steps, and consequently reducing the limitations associated with geometrical design. However, since LS consolidates particles together, porosity of parts may cause leakages under elevated pressures (i.e. powder particles are not normally fully melted). This porosity is caused by the high viscosity of the thermoplastic melts, leading to areas of incomplete particle fusion within the build. It is common practice to use a mixture of fresh powder and recycled powder (i.e. unsintered powder from previous builds); in this research the powder used was $20 \%$ recycled, $80 \%$ virgin, which is well within manufacturer recommendations provided by EOS.

LS is a well-established AM process and consequently repeatability is good when wellmaintained machines are operated by experienced users. Dimensional accuracy is comparable to professional standard FDM machines but not quite as good as SL. Layer thicknesses are usually between 0.1 and $0.2 \mathrm{~mm}$, typically $0.15 \mathrm{~mm}$. Usually, dimensional accuracies of $+/-$ $0.2 \mathrm{~mm}$ are achievable on small parts (e.g., below $200 \mathrm{~mm}$ ). The laser spot size is comparatively large compared to SL at around $0.6 \mathrm{~mm}$ which dictates the minimum wall thickness achievable. LS parts are dimensionally stable and comparatively tough but can be subject to distortion over time. This phenomenon is referred to as creep and is the tendency for solid materials to slowly distort over time when subject to mechanical and environmental stresses.

During the course of this research LS parts were manufactured using an EOS Formiga P100. The P100 has a build platform of $200 \mathrm{~mm}$ x $250 \mathrm{~mm}$ x $330 \mathrm{~mm}$, which is large enough to provide complete design freedom when manufacturing typical reactor parts. Parts can be built at speeds of $20 \mathrm{~mm} / \mathrm{h}$ depending on the processing parameters, build volume, and material being used. Layer thicknesses of $60 \mu \mathrm{m}$ are possible, allowing intricate design features to be manufactured to a high resolution. The laser used is a $30 \mathrm{~W} \mathrm{CO}_{2}$ powered laser, much less powerful than the ones used during SLM processes. LS can be an expensive manufacturing process with this particular machine costing in excess of $£ 200,000$. Parts can be manufactured from a range of commercially available polyamides to a high geometrical resolution. During the course of this research parts were manufactured from 3D systems Duraform PA laser sintering material (Nylon 12). Again this material is expensive to purchase costing in excess of $£ 100 / \mathrm{kg}$. The materials choice was largely due to availability 
and materials cost, however this material is chemically resistant across a wide range of common organic laboratory and may prove a suitable material for some flow applications.

\section{1. $\quad$ Results and Discussion}

\subsubsection{LS Parts 1-3}

\section{Design, build and post processing:}

The first parts manufactured via LS were designed with a large internal reaction volume, which had a helical channel design. Removing any tight corners in the channel design should facilitate powder removal from the part. Three parts were manufactured, varying the channel diameter of each with the dimensions outlined in Table 10.

\begin{tabular}{|c|c|c|c|c|c|c|c|}
\hline $\begin{array}{c}\text { Part } \\
\text { Number }\end{array}$ & $\begin{array}{c}\text { Height } \\
(\mathbf{m m})\end{array}$ & $\begin{array}{c}\text { Width } \\
(\mathbf{m m})\end{array}$ & $\begin{array}{c}\text { Length } \\
(\mathbf{m m})\end{array}$ & $\begin{array}{c}\text { Volume } \\
\left(\mathbf{c m}^{\mathbf{3}}\right)\end{array}$ & $\begin{array}{c}\text { Channel } \\
\text { Length } \\
(\mathbf{m m})\end{array}$ & $\begin{array}{c}\text { Channel } \\
\text { Diameter } \\
(\mathbf{m m})\end{array}$ & $\begin{array}{c}\text { Internal } \\
\text { Reaction } \\
\text { Volume } \\
\left(\mathbf{c m}^{\mathbf{3}}\right)\end{array}$ \\
\hline 1 & 89.10 & 62.00 & 62.00 & 168.90 & 6500 & 1.00 & 5.11 \\
\hline 2 & 89.10 & 62.00 & 62.00 & 168.90 & 6500 & 1.50 & 11.49 \\
\hline 3 & 89.10 & 62.00 & 62.00 & 168.90 & 6500 & 2.00 & 20.43 \\
\hline
\end{tabular}

Table 10: SL Parts 1-3 Part Dimensions. 


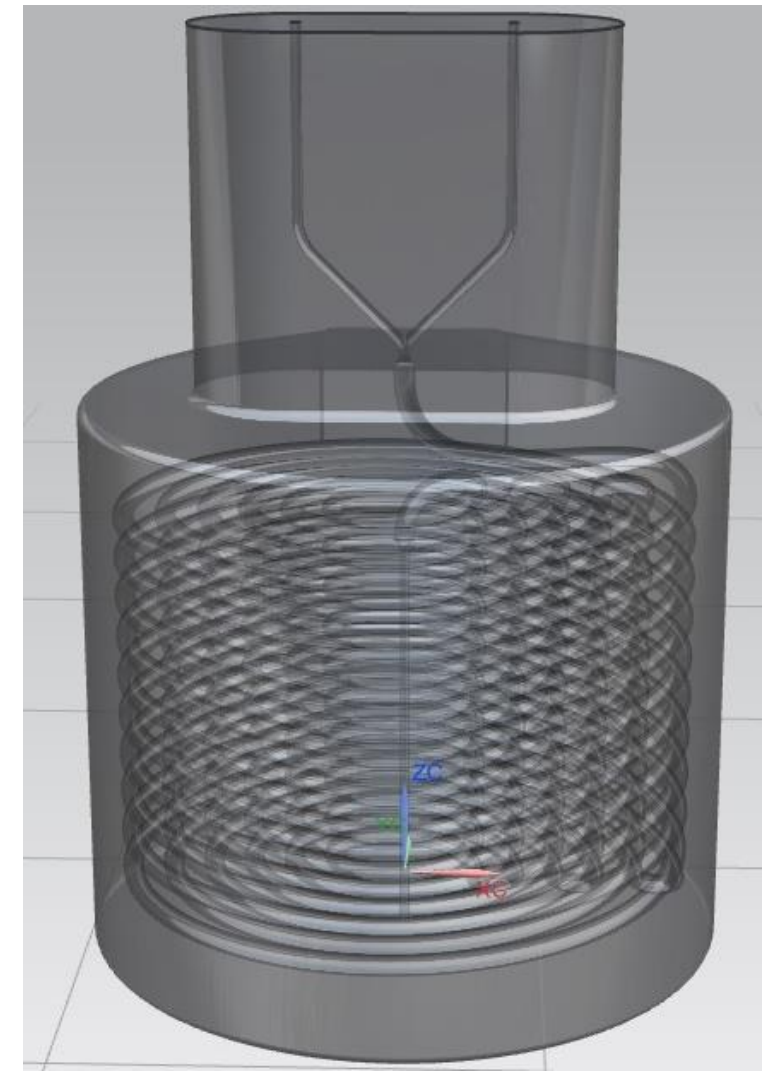

Figure 44: CAD Drawing for SL Parts 1-3.

\section{Application/Discussion:}

Unfortunately, powder removal from these parts was not successful. Unlike SL, where the uncured material was in a liquid form and could be easily drained from channels and voids within the build, the unused LS powder material remained trapped in situ despite attempts to remove it manually by agitation, sonication, compressed air, etc. The part was sliced into sections to investigate the internal structures of the build. It was found that the un-sintered powder had formed a dense semi-solid that was difficult to remove or machine out. It is believed that unwanted partial consolidation of Nylon powder, caused by heat conduction from the internal surface of the tube, lead to some blockages within the reactor channel, preventing powder removal. Semi-crystalline thermoplastics such as Nylon often demonstrate a large degree of shrinkage upon cooling, through both normal thermal shrinkage but also crystallisation processes. Typically this can cause thermoplastics to shrink by as much as a few percent by volume. This shrinkage may have reduced the internal dimensions of the tube, compressing the powder within.

Measurements of the parts were taken in the $\mathrm{x}$ and $\mathrm{y}$ plane, comparing this data with the original CAD to determine dimensional accuracy. 


\begin{tabular}{|c|c|c|c|c|c|}
\hline Part & Dimension & $\begin{array}{c}\text { Actual } \\
\text { Measurement } \\
(\mathbf{m m})\end{array}$ & $\begin{array}{c}\text { CAD } \\
\text { measurement } \\
(\mathbf{m m})\end{array}$ & $\begin{array}{c}\text { Difference } \\
(+/-\mathbf{m m})\end{array}$ & $\begin{array}{c}\text { Variation } \\
(\%)\end{array}$ \\
\hline 1 & $\mathrm{X}$ & 62.19 & 62.00 & 0.19 & 0.31 \\
\hline 2 & $\mathrm{X}$ & 62.36 & 62.00 & 0.36 & 0.58 \\
\hline 3 & $\mathrm{X}$ & 62.16 & 62.00 & 0.16 & 0.26 \\
\hline 1 & $\mathrm{Y}$ & 62.18 & 62.00 & 0.18 & 0.29 \\
\hline 2 & $\mathrm{Y}$ & 62.21 & 62.00 & 0.21 & 0.34 \\
\hline 3 & $\mathrm{Y}$ & 62.18 & 62.00 & 0.18 & 0.29 \\
\hline
\end{tabular}

Table 11: Optical Calliper Measurements of LS Parts 1-3.

A discussion about the accuracy and repeatability of parts built from via the SL process will follow later in the chapter.

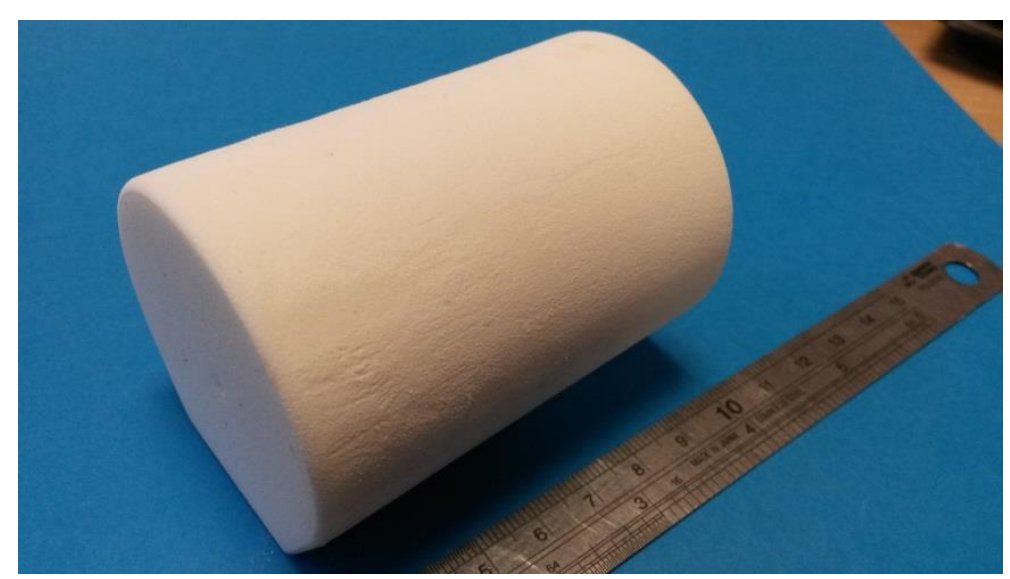

Figure 45: LS Part 3

\subsubsection{LS Parts 4-6}

\section{Design, build and post processing:}

It was hypothesised that powder removal could be facilitated by manufacturing a part with a less complex internal channel network. A design previously used during the manufacture of SL part 2 was reproduced, which had been shown to facilitate resin removal. Three parts were manufactured, varying the channel diameter of each with the dimensions outlined in Table 12. 


\begin{tabular}{|c|c|c|c|c|c|c|c|}
\hline $\begin{array}{c}\text { Part } \\
\text { Number }\end{array}$ & $\begin{array}{c}\text { Height } \\
(\mathbf{m m})\end{array}$ & $\begin{array}{c}\text { Width } \\
(\mathbf{m m})\end{array}$ & $\begin{array}{c}\text { Length } \\
(\mathbf{m m})\end{array}$ & $\begin{array}{c}\text { Volume } \\
\left(\mathbf{c m}^{\mathbf{3}}\right)\end{array}$ & $\begin{array}{c}\text { Channel } \\
\text { Length } \\
(\mathbf{m m})\end{array}$ & $\begin{array}{c}\text { Channel } \\
\text { Diameter } \\
(\mathbf{m m})\end{array}$ & $\begin{array}{c}\text { Internal } \\
\text { Reaction } \\
\text { Volume } \\
\left(\mathbf{c m}^{\mathbf{3}}\right)\end{array}$ \\
\hline 4 & 52.00 & 52.00 & 82.00 & 161.30 & 3240 & 2.00 & 10.18 \\
\hline 5 & 52.00 & 52.00 & 82.00 & 161.30 & 3240 & 2.50 & 15.91 \\
\hline 6 & 52.00 & 52.00 & 82.00 & 161.30 & 3240 & 3.00 & 22.91 \\
\hline
\end{tabular}

Table 12: LS Parts 4-6 Part Dimensions.

\section{Application/Discussion:}

Unfortunately, once again powder removal from these parts was not successful, despite attempts to remove it manually by agitation, sonication, compressed air, etc.

Measurements of the parts were taken in the $\mathrm{x}$ and $\mathrm{y}$ plane, comparing this data with the original CAD to determine dimensional accuracy.

\begin{tabular}{|c|c|c|c|c|c|}
\hline Part & Dimension & $\begin{array}{c}\text { Actual } \\
\text { measurement } \\
(\mathbf{m m})\end{array}$ & $\begin{array}{c}\text { CAD } \\
\text { measurement } \\
(\mathbf{m m})\end{array}$ & $\begin{array}{c}\text { Difference } \\
(+/-\mathbf{m m})\end{array}$ & $\begin{array}{c}\text { Variation } \\
(\%)\end{array}$ \\
\hline 4 & $\mathrm{X}$ & 52.17 & 52.00 & 0.17 & 0.33 \\
\hline 5 & $\mathrm{X}$ & 51.92 & 52.00 & 0.08 & 0.15 \\
\hline 6 & $\mathrm{X}$ & 51.84 & 52.00 & 0.16 & 0.31 \\
\hline 4 & $\mathrm{Y}$ & 51.99 & 52.00 & 0.01 & 0.02 \\
\hline 5 & $\mathrm{Y}$ & 51.95 & 52.00 & 0.05 & 0.10 \\
\hline 6 & $\mathrm{Y}$ & 51.89 & 52.00 & 0.11 & 0.21 \\
\hline
\end{tabular}

Table 13: Optical Calliper Measurements of LS Parts 4-5.

\subsubsection{LS Parts 7-8}

\section{Design, build and post processing:}

The complexity of the previous design was again reduced, with a very short channel being designed. LS parts 7-8 were designed with a very simplistic helical structure, in order to once again facilitate powder removal. Two parts were manufactured, varying the channel diameter of each with the dimensions outlined in Table 14. 


\begin{tabular}{|c|c|c|c|c|c|c|c|}
\hline $\begin{array}{c}\text { Part } \\
\text { Number }\end{array}$ & $\begin{array}{c}\text { Height } \\
(\mathbf{m m})\end{array}$ & $\begin{array}{c}\text { Width } \\
(\mathbf{m m})\end{array}$ & $\begin{array}{c}\text { Length } \\
(\mathbf{m m})\end{array}$ & $\begin{array}{c}\text { Volume } \\
\left(\mathbf{c m}^{\mathbf{3}}\right)\end{array}$ & $\begin{array}{c}\text { Channel } \\
\text { Length } \\
(\mathbf{m m})\end{array}$ & $\begin{array}{c}\text { Channel } \\
\text { Diameter } \\
(\mathbf{m m})\end{array}$ & $\begin{array}{c}\text { Internal } \\
\text { Reaction } \\
\text { Volume } \\
\left(\mathbf{c m}^{\mathbf{3}}\right)\end{array}$ \\
\hline 7 & 26.24 & 60.00 & 60.00 & 71.02 & 720 & 2.50 & 3.54 \\
\hline 8 & 26.24 & 60.00 & 60.00 & 71.02 & 720 & 3.00 & 5.09 \\
\hline
\end{tabular}

Table 14: LS Parts 7-8 Part Dimensions.

\section{Application/Discussion:}

Again un-sintered powder removal from within the channels was unsuccessful. Measurements of the parts were taken in the $\mathrm{x}$ and $\mathrm{y}$ plane, comparing this data with the original CAD to determine dimensional accuracy.

\begin{tabular}{|c|c|c|c|c|c|}
\hline Part & Dimension & $\begin{array}{c}\text { Actual } \\
\text { Measurement } \\
(\mathbf{m m})\end{array}$ & $\begin{array}{c}\text { CAD } \\
\text { measurement } \\
(\mathbf{m m})\end{array}$ & $\begin{array}{c}\text { Difference } \\
(+/-\mathbf{m m})\end{array}$ & $\begin{array}{c}\text { Variation } \\
(\boldsymbol{\%})\end{array}$ \\
\hline 7 & $\mathrm{X}$ & 59.92 & 60.00 & 0.08 & 0.13 \\
\hline 8 & $\mathrm{X}$ & 59.90 & 60.00 & 0.10 & 0.17 \\
\hline 7 & $\mathrm{Y}$ & 59.87 & 60.00 & 0.13 & 0.22 \\
\hline 8 & $\mathrm{Y}$ & 59.76 & 60.00 & 0.24 & 0.40 \\
\hline
\end{tabular}

Table 15: Optical Calliper Measurements of LS Parts 7-8.

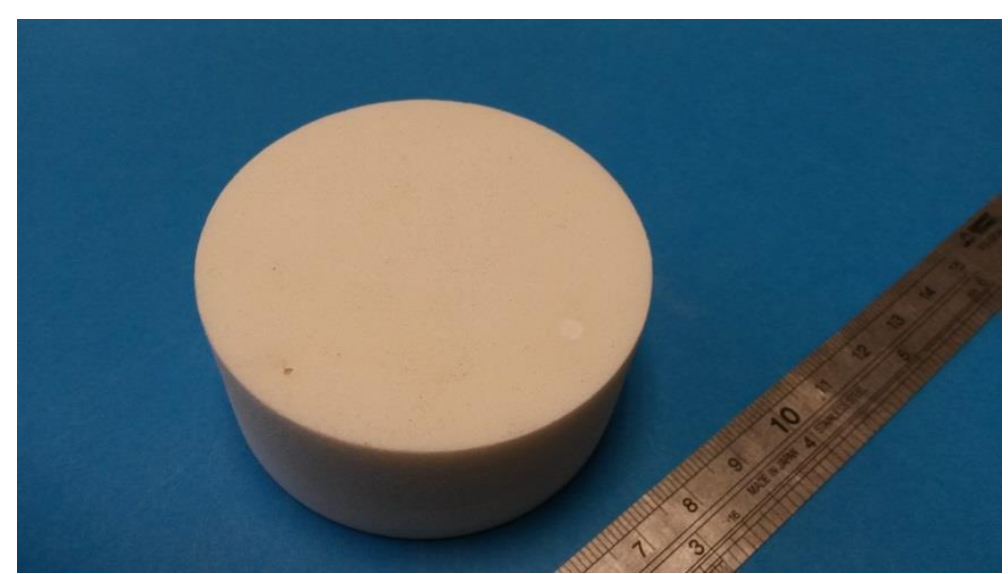

Figure 46: LS Part 7. 


\subsection{4. $\quad$ LS Part 9}

\section{Design, build and post processing:}

To investigate the minimum tube dimension from which powder could be removed from an LS produced part, LS part 9 was designed with 35 linear channels $(50 \mathrm{~mm})$ running throughout the body of the part, with diameters ranging from $1 \mathrm{~mm}$ to $4.5 \mathrm{~mm}$ in $0.1 \mathrm{~mm}$ increments.

\section{Application/Discussion:}

Again it proved difficult to remove the powder from any of the tubes, even including the larger $4.5 \mathrm{~mm}$ diameter tubes. Indeed it required the use of a handheld drill to remove the powder, which had formed a semi-solid material. At this point, it had become clear that the current LS materials, equipment and processing parameters being used, would not allow us to manufacture chemical reactors with milli-scale internal dimensions and complex channel designs.

Measurements of the part were taken in the $\mathrm{x}, \mathrm{y}$ and $\mathrm{z}$ plane, comparing this data with the original $\mathrm{CAD}$ to determine dimensional accuracy.

\begin{tabular}{|c|c|c|c|c|c|}
\hline Part & Dimension & $\begin{array}{c}\text { Actual } \\
\text { Measurement } \\
(\mathbf{m m})\end{array}$ & $\begin{array}{c}\text { CAD } \\
\text { measurement } \\
(\mathbf{m m})\end{array}$ & $\begin{array}{c}\text { Difference } \\
(+/-\mathbf{m m})\end{array}$ & $\begin{array}{c}\text { Variation } \\
(\boldsymbol{\%})\end{array}$ \\
\hline 9 & $\mathrm{X}$ & 59.80 & 60.00 & 0.20 & 0.33 \\
\hline 9 & $\mathrm{Y}$ & 60.01 & 60.00 & 0.01 & 0.02 \\
\hline 9 & $\mathrm{Z}$ & 49.98 & 50.00 & 0.02 & 0.04 \\
\hline
\end{tabular}

Table 16: Optical Calliper Measurements of LS Part 9.

In general, dimensional accuracy of parts built via the LS process from Nylon using a FORMIGA system were excellent. For the nine parts manufactured, dimensions were on average within $+/-132 \mu \mathrm{m}$ of the intended dimensions. This equated to around a $0.225 \%$ discrepancy in geometrical accuracy. 


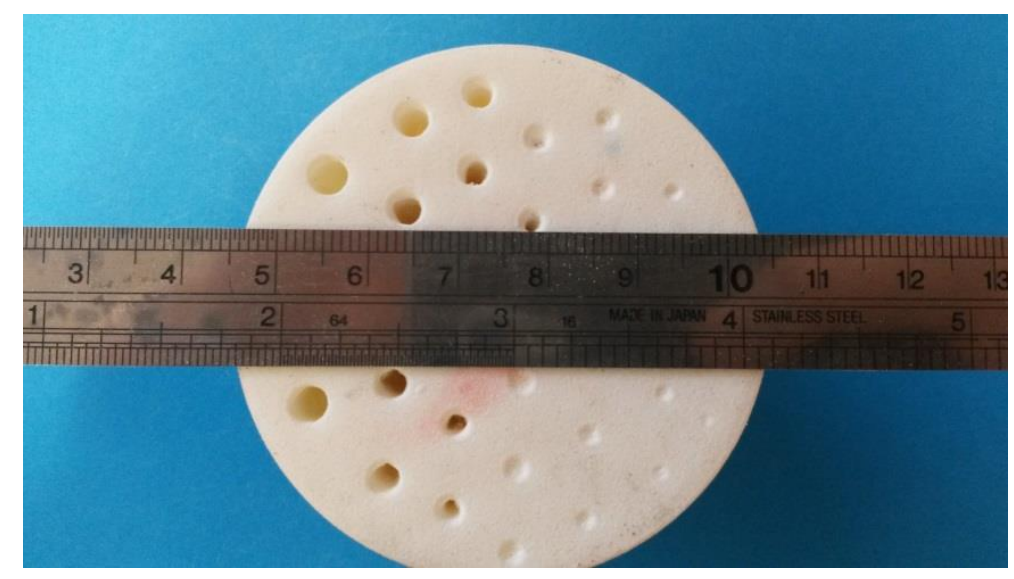

Figure 47: LS Part 9

\subsection{Conclusion}

The difficulty in removing powder from the hollow thermoplastic LS parts, presents a fundamental challenge to the use of this technology for chemical reactor manufacture. However, it is highly possible that these issues could be reduced through the optimisation of processing conditions, further material development and innovative reactor design, leading to LS becoming a highly desirable process for reactor manufacture.

\subsection{Future Work}

Future work in this area will focus on optimising the materials and processing parameters required to facilitate powder removal from within internal channels. This will include research into different materials processable via LS, which may facilitate powder removal from within internal channels. 


\section{5. $\quad$ Stereolithography}

\subsection{Introduction}

Stereolithography (SL) is a technique that was developed in 1986 by 3D systems, which uses layer-by-layer photo-polymerisation of a liquid resin to create solid three-dimensional parts. ${ }^{136}$ Like most AM techniques, the SL process begins with the fabrication of a CAD file. This file is converted to an STL file, which lists the coordinates of triangles that together make up the surface of the designed 3D structure. This design is sliced into layers of a predefined thickness defined by the SL machine settings. These files are then loaded onto the SL software and the structure is fabricated. The fabrication process is based around the solidification of a liquid resin using photo-polymerisation. Using a computer-controlled laser beam or a digital light projector with a computer-driven building stage, a pattern is illuminated on the surface of a resin bath. As a result of this, the resin in the pattern is solidified to a defined depth, causing it to adhere to a support platform. After each layer has been polymerised, the build platform moves down by a predefined layer thickness, and a recoating blade moves over the surface to apply a new layer of resin. These steps are repeated until the part has been completely built. The part is removed from the resin bath and cleaned to remove any uncured resin, before being placed into a high intensity UV chamber to improve the mechanical properties of the part, by ensuring that all of the material is completely cured. ${ }^{137}$

SL is a highly accurate and reproducible manufacturing technique, capable of producing parts at a layer thickness of as little as $0.05 \mathrm{~mm}$ and accuracies of approximately $+/-0.1 \mathrm{~mm}$ (Chapter 5.2.8). Laser spot size can be varied but is typically around $0.2 \mathrm{~mm}$, however the minimum wall thickness achievable in practice is around $0.5 \mathrm{~mm}$. Although capable of thinner layers, larger SL machines are typically operated at thicker layers (0.1 to $0.15 \mathrm{~mm})$ for economic reasons. A well maintained SL machine produces excellent repeatability and reproducibility of manufactured parts. Dimensional accuracy of completed parts can be affected by environmental conditions that the final part is exposed to; prolonged exposure to UV light and excessive humidity can lead to softening and distortion of parts, especially when subject to continued stress (i.e. creep). ${ }^{138}$ This is typically avoided by dry storage and sometimes with the use of protective lacquers. ${ }^{139}$

SL has several significant advantages as a manufacturing process, which makes it an excellent choice for milli-scale reactor manufacture. The superior build resolution should 
allow narrow flow paths and complex internal structures to be realised. The solid part is produced in a bath of the liquid un-cured resin, making the production of parts with hollow sections (required for flow) reasonably simple, with the resin simply draining clear from the channels. In addition, parts have good optical clarity (although opaque resins are available), allowing internal structures and defects to be easily visualised, and reactions to be visually monitored as they proceed. ${ }^{140}$ Unfortunately, SL material choices are typically limited to a narrow range of UV-curable photo-polymers, typically based on acrylates, epoxides and urethanes. Although the final material is likely to be densely cross-linked, conferring better mechanical properties and solvent resistance than many polymers, it is still vulnerable to destructive swelling in some solvents. The commercially available SL resin used in our work, 3D Systems Accura 60, contains a cycloaliphatic diepoxide, polymerised by a photo-cationic initiator, and an aliphatic tetra-acrylate, polymerised by a photoradical initiator. ${ }^{141}$ The cured Accura 60 material may demonstrate swelling upon immersion in a range of organic solvents (Table 17). Although this particular polymer is thermally stable to $150^{\circ} \mathrm{C}$, the heat deflection temperature (the temperature at which a polymer begins to deform under a specified load) is relatively low at around $50^{\circ} \mathrm{C}$ and the material is poorly conducting. ${ }^{142}$ Above this temperature the polymer becomes less rigid and may not be suitable for high-pressure work. This limits the range of chemistry available when using Accura 60 parts to low temperature reactions in milder solvent systems. However, research is on-going to develop new SL materials with better chemical and mechanical properties, with materials now available which are comparable to commercially used polymers such as ABS, PC and PEEK. ${ }^{142}$ Ceramic filled resins are also available which offer excellent mechanical properties and increased resistance to solvents, although their comparatively high viscosity means that very narrow channels become difficult to clear. ${ }^{140}$

During the course of this research all SL parts were manufactured using a Viper si2 SL system. The Viper system uses a solid state neodymium-doped yttrium orthovanadate $\left(\mathrm{Nd}: \mathrm{YVO}_{4}\right)$ laser at wavelength of $354.7 \mathrm{~nm}$, to generate up to $100 \mathrm{~mW}$ of power at the surface of the vat. ${ }^{142}$ The laser generated has a typical beam diameter of $0.25+/-0.025 \mathrm{~mm}$, but can be set to a high resolution mode whereby the laser has a beam diameter of $0.075+/-$ $0.015 \mathrm{~mm}$. The build platform is $250 \times 250 \times 250 \mathrm{~mm}$ in standard mode, and $125 \times 125 \times 125$ $\mathrm{mm}$ in high resolution mode. During the course of this research for economic reasons all parts are produced using the standard mode settings. The machine is run through its own inbuilt software Buildstation 5.3. ${ }^{131}$ 


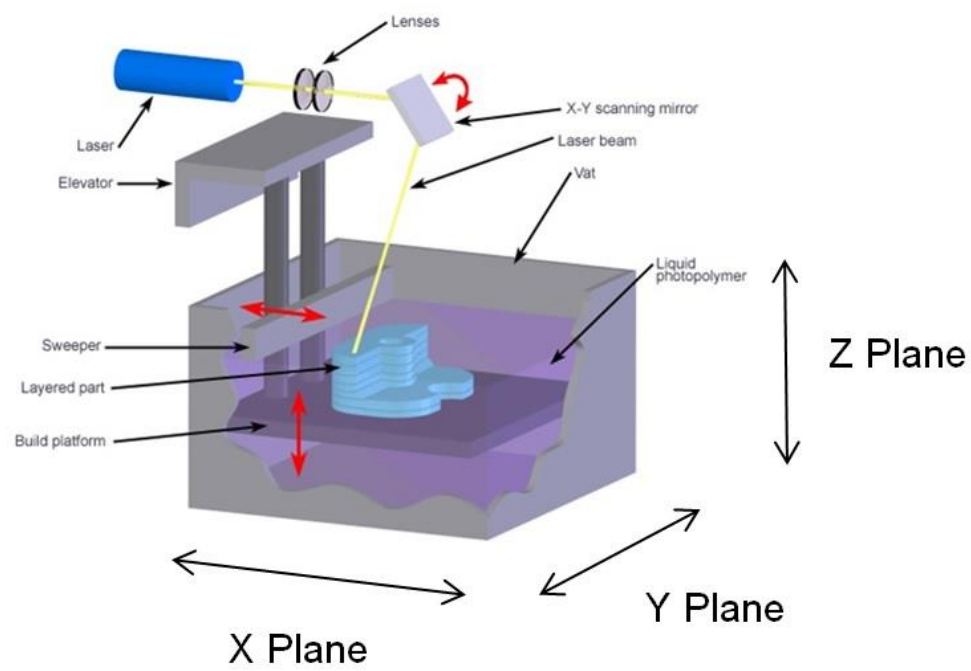

Figure 48: SL build process. ${ }^{143}$

\subsection{Results and Discussion}

During the course of this research all SL parts were manufactured from Accura 60 resin. The solvent compatibility of the Accura 60 material was determined as follows (Table 17). $1 \mathrm{~cm}^{3}$ blocks of Accura 60 resin were manufactured before being exposed (submerged in a stirred solution) to a range of common laboratory solvents. Each cube was weighed before and after exposure, with the weight gain/loss through swelling being quantified. Notes were also added where any visual observations were made. Variations in dimensions due to swelling were also recorded, using callipers to conduct the measurements. Initial variations in weights are a reflection of the effectiveness of the support structure removal process, rather than the accuracy of the build, which often lead to a thin layer of material being left on the underside of the parts. All dimensional measurements were conducted in the z plane of the build. Slight degradation (SD) of parts was defined as any visible damage to the surface of the part that did not alter the overall shape of the cube. Total degradation (TD) was defined as any visible damage to the surface of the part that did alter the overall shape of the cube. Delamination was defined as swelling that lead to individual layers of the part delaminating from one another. Colour changes were defined as any loss or change of colouring in the part (CC). 


\begin{tabular}{|c|c|c|c|c|c|c|c|c|}
\hline Solvent & $\begin{array}{c}\text { Initial } \\
\text { Weight } \\
\text { (g) }\end{array}$ & $\begin{array}{l}\text { Weight } \\
\text { after } 30 \\
\min (g)\end{array}$ & $\begin{array}{c}\text { Weight } \\
\text { Change } \\
(\%)\end{array}$ & $\begin{array}{l}\text { Weight } \\
\text { after } 60 \\
\min (g)\end{array}$ & $\begin{array}{c}\text { Weight } \\
\text { Change } \\
(\%)\end{array}$ & $\begin{array}{c}\text { Weight } \\
\text { after } 24 \\
\text { (g) }\end{array}$ & $\begin{array}{c}\text { Weight } \\
\text { Change } \\
(\%)\end{array}$ & $\begin{array}{c}\text { Dimensional } \\
\text { Changes (+/- } \\
\text { mm) }\end{array}$ \\
\hline $\begin{array}{c}\text { Ethyl } \\
\text { acetate }\end{array}$ & 1.2632 & 1.2636 & 0.03 & 1.2676 & 0.35 & 1.2826 & 1.54 & 0.09 \\
\hline $\begin{array}{l}\text { Diethyl } \\
\text { ether }\end{array}$ & 1.2651 & 1.2646 & 0.04 & 1.2652 & 0.01 & 1.2712 & 0.48 & 0.09 \\
\hline Toluene & 1.2729 & 1.2733 & 0.03 & 1.2721 & 0.09 & 1.2740 & 0.09 & 0.14 \\
\hline $\mathrm{CH}_{2} \mathrm{Cl}_{2}$ & 1.2687 & 1.3047 & 2.83 & SD & N/A & TD & N/A & N/A \\
\hline $\mathrm{THF}$ & 1.2623 & 1.2715 & 0.73 & 1.2928 & 2.42 & SD & N/A & N/A \\
\hline $\begin{array}{c}\text { Light } \\
\text { petroleum }\end{array}$ & 1.2832 & 1.2828 & 0.03 & 1.2820 & 0.09 & 1.2820 & 0.09 & 0.09 \\
\hline Methanol & 1.2673 & 1.2788 & 0.91 & 1.3021 & 2.75 & SD & N/A & N/A \\
\hline$i \mathrm{PrOH}$ & 1.2625 & 1.2622 & 0.02 & 1.2632 & 0.06 & 1.2740 & 0.91 & 0.07 \\
\hline Hexane & 1.2773 & 1.2774 & 0.01 & 1.2767 & 0.05 & 1.2771 & 0.01 & 0.08 \\
\hline $\begin{array}{c}\text { Butan-1- } \\
\text { ol }\end{array}$ & 1.2739 & 1.2739 & 0.00 & 1.2720 & 0.15 & 1.2765 & 0.20 & 0.11 \\
\hline
\end{tabular}

Table 17: Solvent compatibility data for the Accura 60 SL material, in a range of common organic solvents.

The Accura material proved to be highly susceptible to degradation in dichloromethane, tetrahydrofuran and methanol. Some degredation was observed in solvents such as ethyl acetate, butan-1-ol, isopropanol and diethyl ether. Limited degradation was observed in hexane, light petroleum and toluene. 


\subsubsection{SL Part 1}

\section{Design, build and post processing:}

For the first part that was designed and manufactured using SL, the design criteria were very simplistic. SL part 1 was intended purely as a proof of concept prototype, to demonstrate the capability of SL to build a simplistic tube capable of flowing liquids through. The part needed to have a short channel running through the material, featuring some smooth curves with tight corners (small radius of curvature). This would not only test the ability of the Viper machine to accurately produce the part, but also to determine whether resin removal from the channels would be possible. The part was therefore designed with a helical channel network with the internal and external dimensions outlined in Table $\mathbf{1 8 .}$

\begin{tabular}{|c|c|}
\hline Part Length $(\mathrm{mm})$ & 40.00 \\
\hline Part Width $(\mathrm{mm})$ & 40.00 \\
\hline Part Height $(\mathrm{mm})$ & 72.50 \\
\hline Part Volume $\left(\mathrm{cm}^{3}\right)$ & 113.70 \\
\hline Channel Diameter $(\mathrm{mm})$ & 2.00 \\
\hline Channel Length $(\mathrm{mm})$ & 439 \\
\hline Internal Reaction Volume $\left(\mathrm{cm}^{3}\right)$ & 1.38 \\
\hline
\end{tabular}

Table 18: SL part 1 Build Dimensions.

The uncured resin was easily removed from within the channels, by repeatedly washing with streams of isopropanol dispensed from a wash bottle. The part was tapped at each end of the channel with a 10-32 UNF screw thread. Having tapped the part, a PTFE o-ring was inserted into both threaded holes to allow a better seal to be generated between the part and the standard PEEK fittings used by most flow chemistry apparatus. 


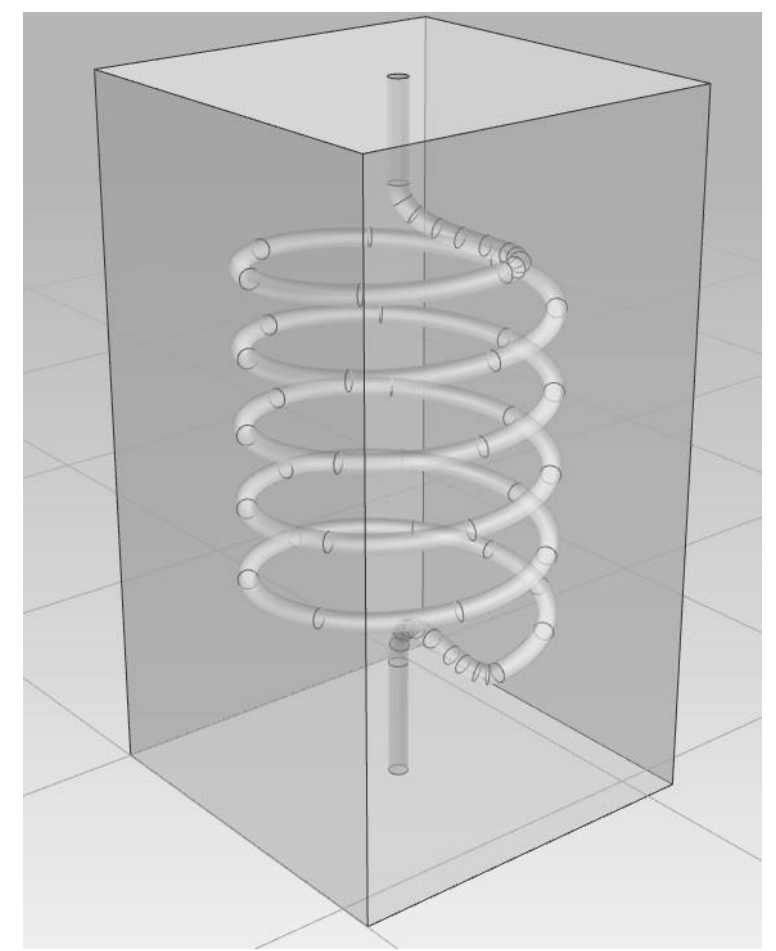

Figure 49: CAD model of SL part 1.

\section{Application/Discussion:}

A range of common organic solvents $(\mathrm{MeOH}$, IPA, water, petroleum ether) were pumped through the part using the Uniqsis FlowSyn at flow rates ranging from $0.1-5 \mathrm{~mL} / \mathrm{min}$, confirming that the part would not leak under elevated pressures ( $<20 \mathrm{bar})$. Although the part was not used for any chemical reactions, it demonstrated the applicability of the SL process towards manufacturing relatively complex internal channel networks. It also demonstrated the machinability of the Accura 60 material. Although the material was slightly brittle, it was possible to apply a thread to the part, so that it could be connected to commercially available HPLC fittings. This brittleness lead to some parts of the thread being removed/incomplete, which may make it difficult to apply finer threads to this material in the future. 


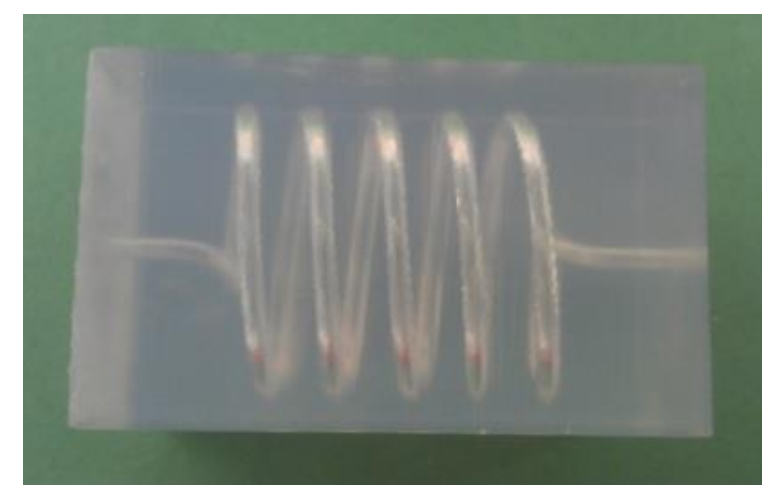

Figure 50: Photograph of SL Part 1 after cleaning.

Measurements of the part were taken in the $\mathrm{x}, \mathrm{y}$ and $\mathrm{z}$ plane, comparing this data with the original CAD to determine dimensional accuracy.

\begin{tabular}{|c|c|c|c|c|}
\hline Dimension & $\begin{array}{c}\text { Actual Measurement } \\
(\mathbf{m m})\end{array}$ & $\begin{array}{c}\text { CAD measurement } \\
(\mathbf{m m})\end{array}$ & $\begin{array}{c}\text { Difference (+/- } \\
\mathbf{m m})\end{array}$ & $\begin{array}{c}\text { Variation } \\
(\boldsymbol{\%})\end{array}$ \\
\hline $\mathrm{x}$ & 40.01 & 40.00 & 0.01 & 0.03 \\
\hline $\mathrm{y}$ & 40.02 & 40.00 & 0.02 & 0.05 \\
\hline $\mathrm{z}$ & 72.49 & 72.50 & 0.01 & 0.01 \\
\hline
\end{tabular}

Table 19: $x, y$ and $\mathrm{z}$ calliper measurements of SL Part 1.

A discussion about the accuracy and repeatability of parts built from Accura 60 via the SL process using a Viper SL system will follow later in the chapter.

\subsubsection{SL Part 2}

\section{Design, build and post processing:}

Having demonstrated that it was possible to manufacture a continuous flow channel, the next progressive step would be to produce a functional part with a much larger internal reaction volume. The solvent compatibility studies that had previously been conducted, demonstrated SL reactors should be able to perform room temperature chemistry with mild solvent systems. The part was designed with another helical channel network of the following dimensions. 


\begin{tabular}{|c|c|}
\hline Part Length $(\mathrm{mm})$ & 52.00 \\
\hline Part Width $(\mathrm{mm})$ & 52.00 \\
\hline Part Height $(\mathrm{mm})$ & 77.00 \\
\hline Part Volume $\left(\mathrm{cm}^{3}\right)$ & 161.30 \\
\hline Channel Diameter $(\mathrm{mm})$ & 3.00 \\
\hline Channel Length $(\mathrm{mm})$ & 3240 \\
\hline Internal Reaction Volume $\left(\mathrm{cm}^{3}\right)$ & 22.91 \\
\hline
\end{tabular}

Table 20: SL Part 2 Build Dimensions.

This helical structure, by removing any tight corners, would theoretically allow us to remove the un-cured resin from the channels more easily. This proved to be the case, with the uncured resin being removed from within the channels using a compressed air line, before repeatedly washing the channels with streams of isopropanol. Although the resin was removed successfully, the length of the channel made this process quite labour intensive; longer channels, with smaller internal features, may prove difficult to clear. The reactor was again tapped at each end using a 10-32 UNF screw thread. The completed reactor was connected to a Uniqsis FlowSyn and flushed continuously with isopropanol for 30 minutes, until all un-cured resin residues had been visibly removed from the system.

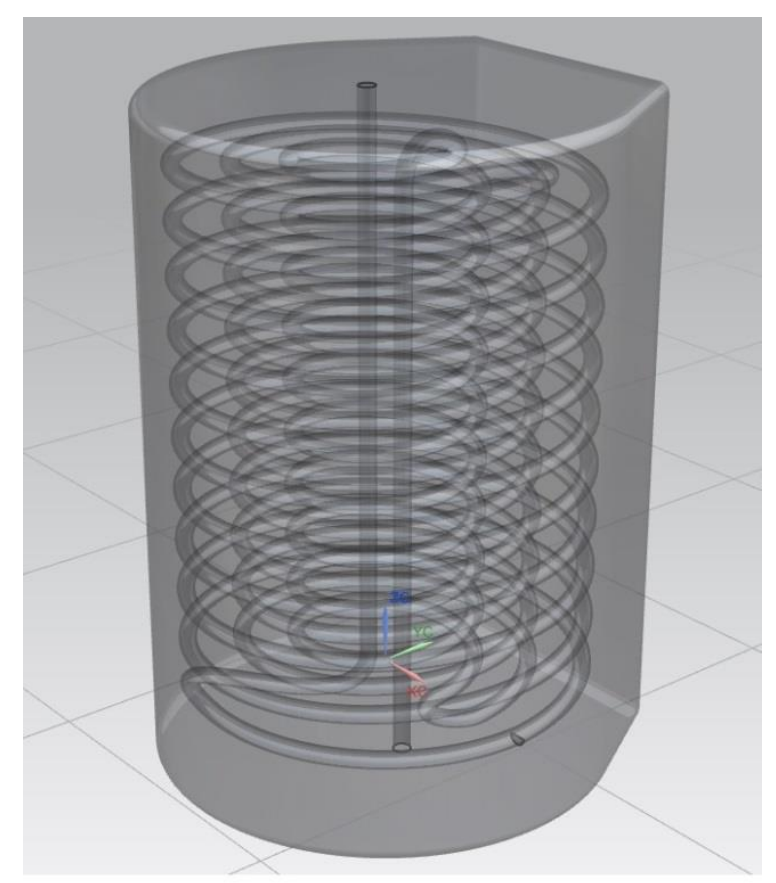

Figure 51: CAD model of SL part 2. 


\section{Application/Discussion:}

In 2009, Jamison et al. demonstrated the room temperature oxidation of aldehydes to their corresponding esters in flow, using ethyl acetate as the solvent and sodium hypochlorite as the oxidising agent (Scheme 1). ${ }^{144}$ These reaction conditions will be reasonably compatible with the Accura 60 SL resin from which SL part 2 had been manufactured. The reaction progression would also be easily confirmed by the disappearance of the characteristic aldehyde peak on the NMR spectra. It was decided that to replicate the studies of Jamison et al., a number of these conversions should be tested. Both studies will use the same FlowSyn continuous flow reaction system, however our SL built reactor will replace the stainless steel coil reactor, allowing us to directly compare results (Table 21). Conversions were taken directly from crude ${ }^{1} \mathrm{H}$ NMR spectra, whereas yields were calculated after workup by mass of the product regained.

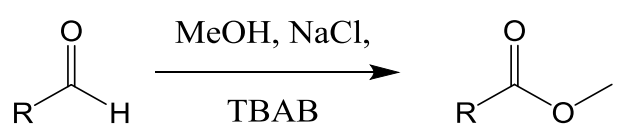

Scheme 1: Reaction conditions used during the oxidation of an aldehyde to a methyl ester. 


\begin{tabular}{|c|c|c|c|c|c|}
\hline \multirow{2}{*}{$\begin{array}{c}\text { Experiment } \\
\text { Number }\end{array}$} & \multirow{2}{*}{ Product } & \multicolumn{2}{|c|}{ Conversion (\%) } & \multicolumn{2}{|c|}{ Yield (\%) } \\
\hline & & $\begin{array}{l}\text { AM } \\
\text { part }\end{array}$ & $\begin{array}{l}\text { Coil reactor } \\
\text { (Jamison) }\end{array}$ & $\begin{array}{l}\text { AM } \\
\text { part }\end{array}$ & $\begin{array}{l}\text { Coil reactor } \\
\text { (Jamison) }\end{array}$ \\
\hline 1 & Methyl Benzoate & 93 & 58 & 86 & 51 \\
\hline 2 & Methyl p-toluate & 54 & 56 & 50 & 45 \\
\hline 3 & $\begin{array}{c}\text { Methyl p- } \\
\text { nitrobenzoate }\end{array}$ & 100 & 100 & 99 & $>95$ \\
\hline 4 & $\begin{array}{c}\text { Methyl p- } \\
\text { bromobenzoate }\end{array}$ & 63 & 85 & 52 & 81 \\
\hline 5 & $\begin{array}{l}\text { Methyl m- } \\
\text { nitrobenzoate }\end{array}$ & 99 & 100 & 99 & 95 \\
\hline 6 & $\begin{array}{l}\text { Methyl Trans- } \\
\text { cinnamate }\end{array}$ & 100 & 96 & 98 & 41 \\
\hline 7 & Methyl Isobutyrate & 100 & N/A & 88 & N/A \\
\hline 8 & Methyl Anisate & 56 & 57 & 34 & 8 \\
\hline
\end{tabular}

Table 21: List of conditions and results of oxidation reactions. tR for all reactions reported was 30 minutes.

The results obtained from the 8 examples which were tested were comparable to those obtained by Jamison et al., demonstrating the potential of performing organic reactions within an SL built reactor, and gain results comparable to those obtained in a conventional tube reactor. Over time it was noticeable that the part was beginning to demonstrate some slight swelling around the channels, however this did not appear to affect its performance under these conditions. The part also began to discolour over time with exposure to UV radiation, a feature associated with many SL materials. 


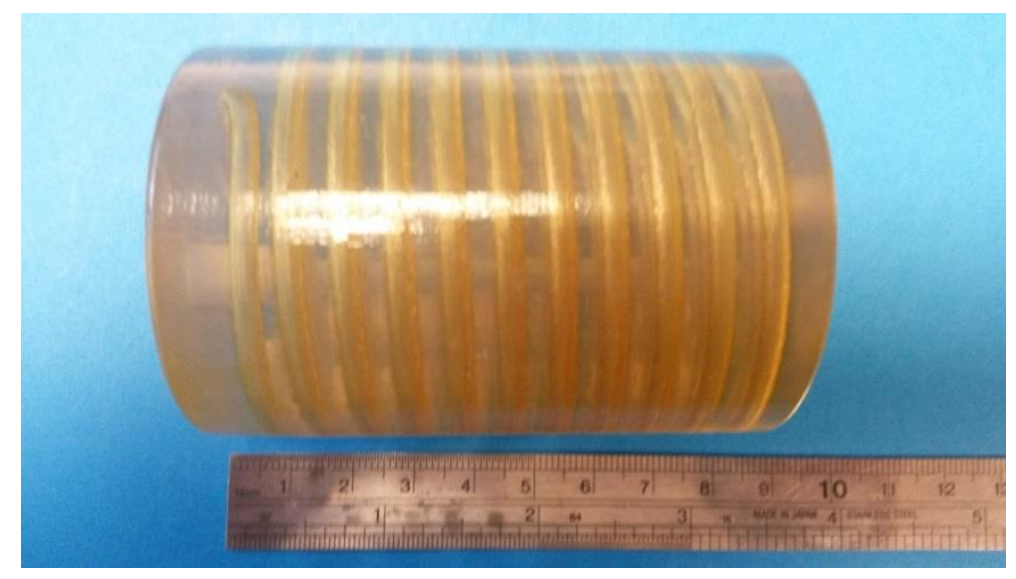

Figure 52: Dis-colouration of SL part 2 upon prolonged UV exposure.

Measurements of the part were taken in the $\mathrm{x}, \mathrm{y}$ and $\mathrm{z}$ plane, comparing this data with the original CAD to determine dimensional accuracy.

\begin{tabular}{|c|c|c|c|c|}
\hline Dimension & $\begin{array}{c}\text { Actual Measurement } \\
(\mathbf{m m})\end{array}$ & $\begin{array}{c}\text { CAD measurement } \\
(\mathbf{m m})\end{array}$ & $\begin{array}{c}\text { Difference (+/- } \\
\mathbf{m m})\end{array}$ & $\begin{array}{c}\text { Variation } \\
(\%)\end{array}$ \\
\hline $\mathrm{X}$ & 52.02 & 52.0 & 0.02 & 0.04 \\
\hline $\mathrm{Y}$ & 52.01 & 52.0 & 0.01 & 0.02 \\
\hline $\mathrm{Z}$ & 76.91 & 77.0 & 0.09 & 0.12 \\
\hline
\end{tabular}

Table 22: Optical Calliper Measurements of SL Part 2.

\subsubsection{SL Part 3}

\section{Design, build and post processing:}

Having demonstrated that SL could be used to manufacture a functioning continuous flow reactor, the next logical progression within our SL development would be to perform a twostep reaction within a single reactor. This would begin to utilise the design freedom associated with AM, with the future possibility of performing multi-step reactions within the same vessel. The design criteria for the build were similar to those of SL part 2, however a second inlet would be placed into the reactor at exactly half way along the reaction volume. The reactor was cleaned and tapped as before. The part was built with the dimensions outlined in Table 23. 


\begin{tabular}{|c|c|}
\hline Part Length $(\mathrm{mm})$ & 52.00 \\
\hline Part Width $(\mathrm{mm})$ & 52.00 \\
\hline Part Height $(\mathrm{mm})$ & 82.00 \\
\hline Part Volume $\left(\mathrm{cm}^{3}\right)$ & 161.30 \\
\hline Channel Diameter $(\mathrm{mm})$ & 3.00 \\
\hline Channel Length $(\mathrm{mm})$ & 3240 \\
\hline Internal Reaction Volume $\left(\mathrm{cm}^{3}\right)$ & 23.00 \\
\hline
\end{tabular}

Table 23: SL Part 3 Build Dimensions.

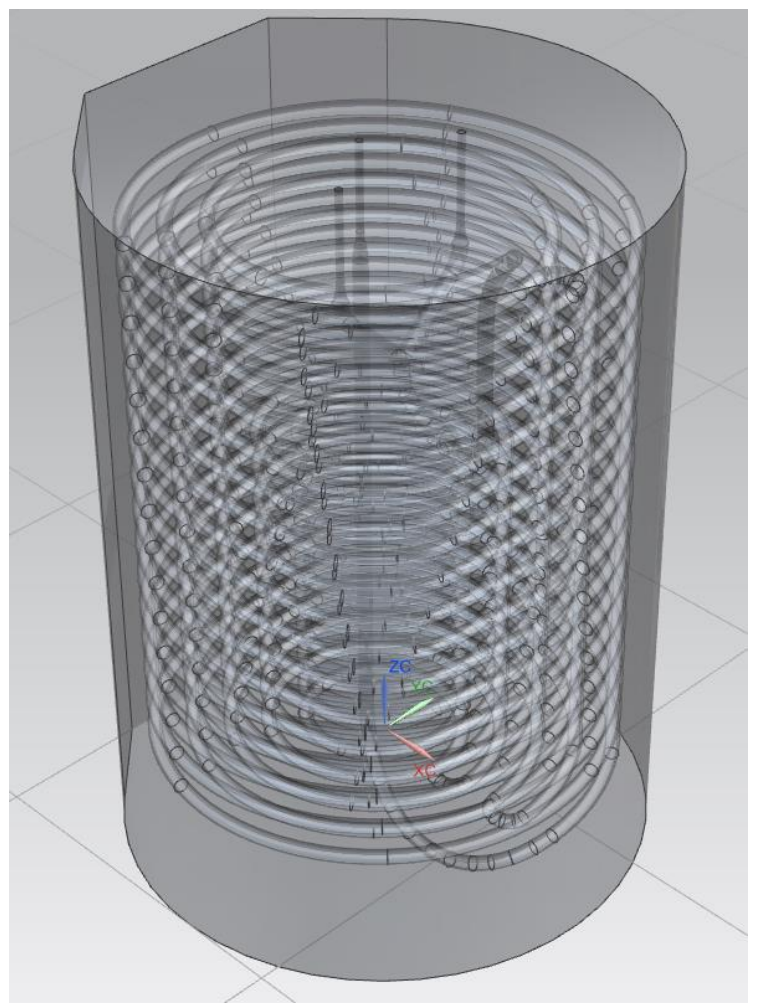

Figure 53: CAD model of SL Part 3

\section{Application/Discussion:}

The reaction selected needed to be a two-step room temperature reaction, ideally previously demonstrated in flow to allow comparison. An appropriate reaction was determined as being the two step synthesis of Sudan 1, a red food dye. The reaction was demonstrated in flow in 2002 by Wooton et al., ${ }^{145}$ who carried out the reaction using a commercially available glass microreactor (Scheme 2). 


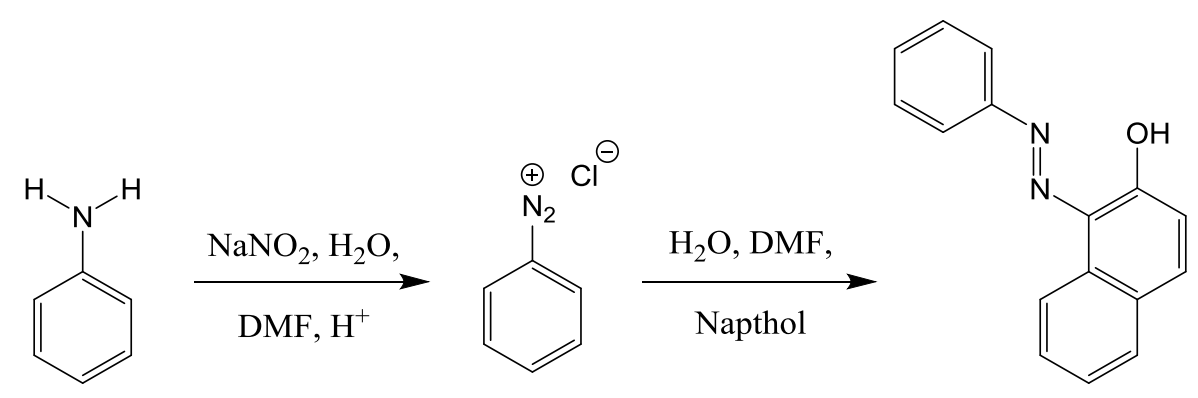

Scheme 2: Reaction conditions for the formation of the diazo dye Sudan-1. ${ }^{145}$

Initially the amine group is converted to an azide salt. Sodium nitrite and hydrochloric acid $(\mathrm{HCl})$ are added together to generate the extremely reactive nitrosonium cation intermediate, which will then react with the amine to produce the arenediazonium salt. The electron rich aromatic ring then attacks the electrophilic nitrogen on the diazonium salt to form the diazolinked aromatic system. The reaction was successfully carried out in our reactor with a $17 \%$ conversion. This reaction may also be of use for future work involving UV-Vis spectroscopic analysis of the reaction, due to the generation of a highly coloured reaction product.

Although the reaction conversion was relatively poor, probably due to lack of optimisation of the flow rates and residence times of the reaction, it demonstrated that a multi-step reaction could be performed within a single reactor. Typically, this reaction can be performed in flow at a conversion of around 50\%. Improvements in the reaction yield could have been made by altering the flow rates to allow the reaction to go to completion as each stage, potentially monitoring the generation of the product at each point in the flow cycle. As well as this, more precise control over the reaction thermodynamics could have been achieved by implementing regions of thermal control within the reactor. ${ }^{146}$ Unfortunately, the reaction conditions proved to be more damaging to the Accura 60 material than initially predicted, and the part swelled destructively (Figure 54). This meant it was not possible to optimise the reaction conditions without rebuilding the part, so despite this initially promising result in order to progress the project into other areas the part was not rebuilt. 


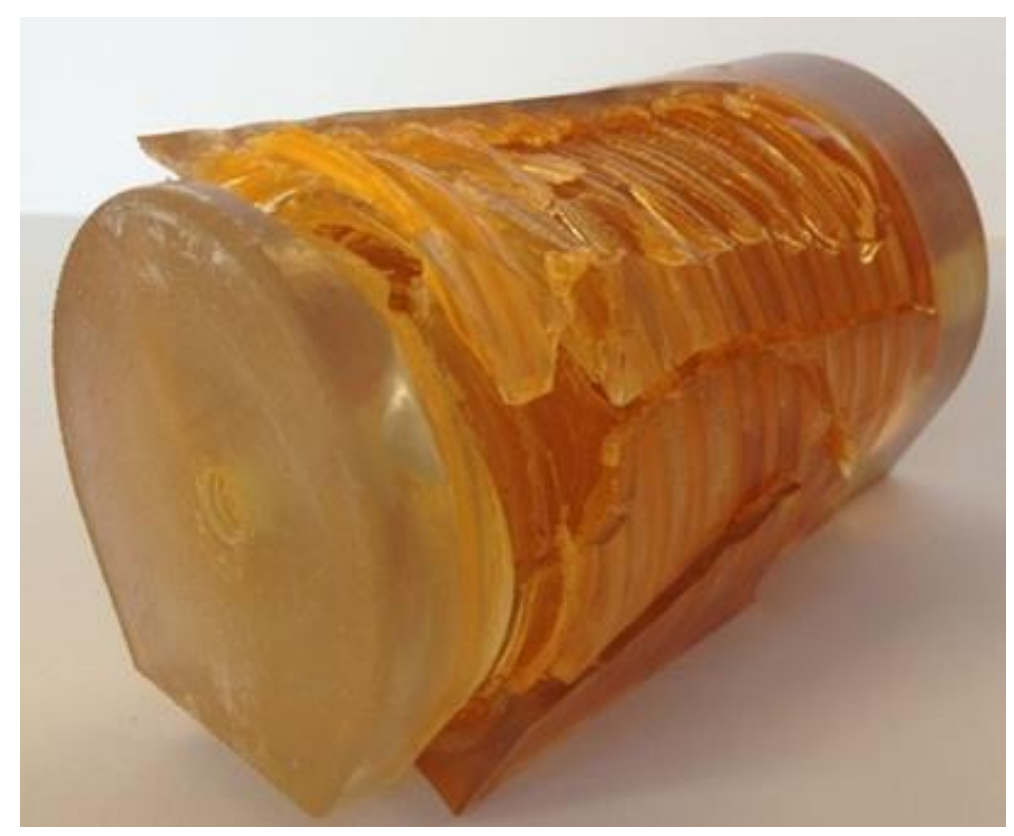

Figure 54: Destructive swelling of SL part 3

Measurements of the part were taken in the $\mathrm{x}, \mathrm{y}$ and $\mathrm{z}$ plane, comparing this data with the original CAD to determine dimensional accuracy.

\begin{tabular}{|c|c|c|c|c|}
\hline Dimension & $\begin{array}{c}\text { Actual Measurement } \\
(\mathbf{m m})\end{array}$ & $\begin{array}{c}\text { CAD measurement } \\
(\mathbf{m m})\end{array}$ & $\begin{array}{c}\text { Difference (+/- } \\
\mathbf{m m})\end{array}$ & $\begin{array}{c}\text { Variation } \\
(\%)\end{array}$ \\
\hline $\mathrm{x}$ & 52.07 & 52.0 & 0.07 & 0.13 \\
\hline $\mathrm{y}$ & 52.03 & 52.0 & 0.03 & 0.06 \\
\hline $\mathrm{z}$ & 76.99 & 77.0 & 0.01 & 0.01 \\
\hline
\end{tabular}

Table 24: Optical Calliper Measurements of SL Part 3.

\subsubsection{SL Part 4}

\section{Design, build and post processing:}

The next development stage of the SL design process was to produce a part that was believed impossible to manufacture by conventional subtractive manufacturing processes, without an unrealistic number of production steps or joining of many separate components. One possibility was to build a reactor with a highly complex channel network. SL part 4 was designed as a large split-and-recombine (SAR) type mixer, ${ }^{147}$ with an extended flow path wrapping around the mixer in a helical fashion. This type of mixer has been shown to substantially improve mixing efficiency within a microfluidic system by increasing the 
intimate contact area between the reaction reagents. This reactor was built with the dimensions outlined in Table 25.

\begin{tabular}{|c|c|}
\hline Part Length $(\mathrm{mm})$ & 50.00 \\
\hline Part Width $(\mathrm{mm})$ & 50.00 \\
\hline Part Height $(\mathrm{mm})$ & 140.00 \\
\hline Part Volume $\left(\mathrm{cm}^{3}\right)$ & 159.20 \\
\hline Channel Diameter $(\mathrm{mm})$ & 3.00 \\
\hline Channel Length $(\mathrm{mm})$ & 3220 \\
\hline Internal Reaction Volume $\left(\mathrm{cm}^{3}\right)$ & 16.40 \\
\hline
\end{tabular}

Table 25: SL Part 4 Build Dimensions.

The reactor was again tapped at each end using a 10-32 UNF screw thread.

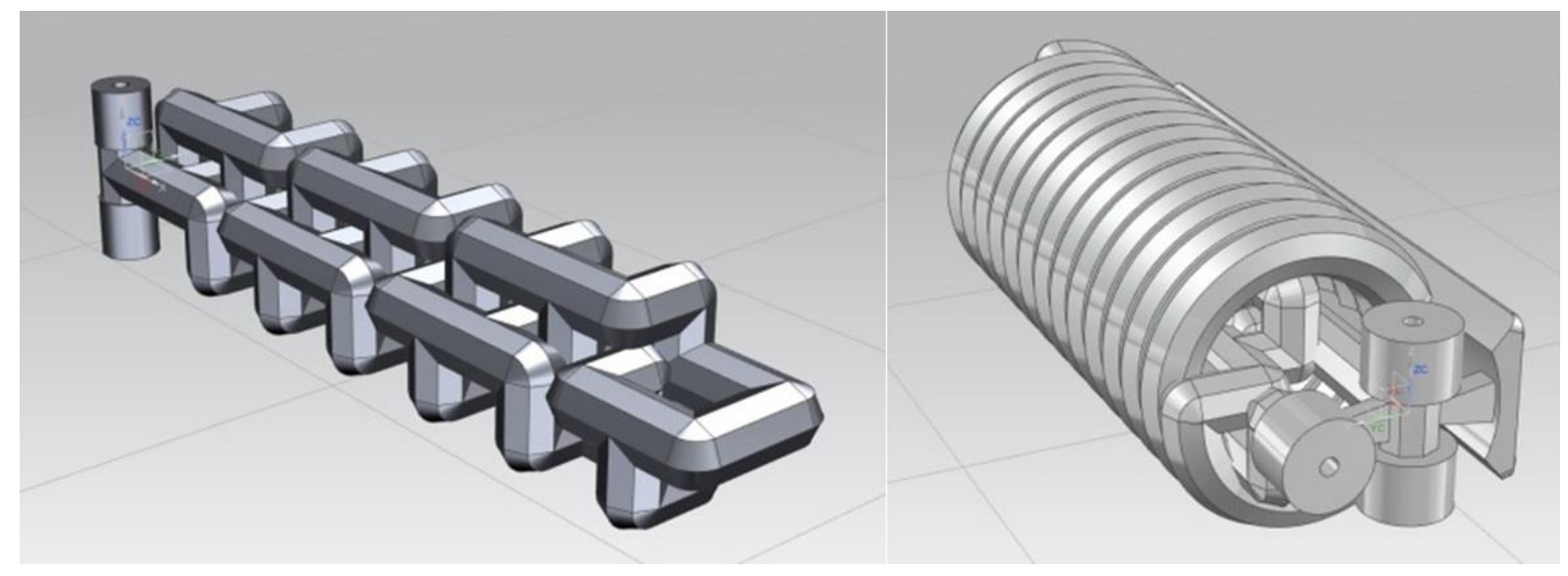

Figure 55: Right) CAD model of SL part 4, left) Internal mobius type mixer used in SL part 4.

\section{Application/Discussion:}

The efficiency of the mixer was experimentally determined via a competing parallel reaction. The chemical reaction that takes places is a redox reaction, whereby potassium iodide is oxidised to elemental iodine, whilst potassium persulfate is reduced (Scheme 3). Once the thiosulfate is consumed the elemental iodine will complex to a starch buffer within the reaction, leading to an instantaneous colour change from colourless to dark blue. ${ }^{148}$

$$
\mathrm{I}^{-}{ }_{\text {(aq.) }}+\mathrm{S}_{2} \mathrm{O}_{8}{ }^{2-}{ }_{\text {(aq.) }} \rightarrow \mathrm{I}_{2}+2 \mathrm{SO}_{4}{ }^{2-}{ }_{\text {(aq.) }}
$$

Scheme 3: The ionic equation for the competing parallel reaction used to determine mixing efficiency within SL part 4. 
However, without a delaying mechanism, the formation of the starch complex would be instantaneous. This delaying mechanism is caused by the addition of sodium thiosulfate, which converts the elemental iodine back to the iodide ion. Once the sodium thiosulfate has been consumed the iodine will be free to form the blue/black starch complex.

$$
\mathrm{I}_{2 \text { (aq.) }}+2 \mathrm{~S}_{2} \mathrm{O}_{3}{ }^{2-} \text { (aq.) } \rightarrow \mathrm{I}^{-} \text {(aq.) }+\mathrm{S}_{2} \mathrm{O}_{6}{ }^{2-} \text { (aq.) }
$$

Scheme 4: The ionic equation for the competing parallel reaction used to determine mixing efficiency within SL part 4.

The time taken for the colour change to occur is indirectly proportional to the rate of the reaction, which can be controlled by altering either the reaction temperature or reagent concentrations. However, if both of these variables are held constant then the reaction rate becomes a function of efficient molecular interaction. Improvements in the molecular interactions within the medium can be realised by improvements in the mixing efficiency of the system. Therefore under these conditions mixing efficiency within the system can be treated as proportional to the time taken for this colour change to occur. The reaction was undertaken in both SL part 4 and PTFE tubing of comparative dimensions across a number of flow rates. Inlet A was charged with a solution of potassium iodide, whilst Inlet B was charged with a solution of potassium persulfate, sodium thiosulfate and starch indicator. When comparing the times to colour change between the two systems it was shown that mixer SL part 4 improved mixing efficiency, as measured by a decrease in reaction time, by as much as 17\% (Figure 56). 


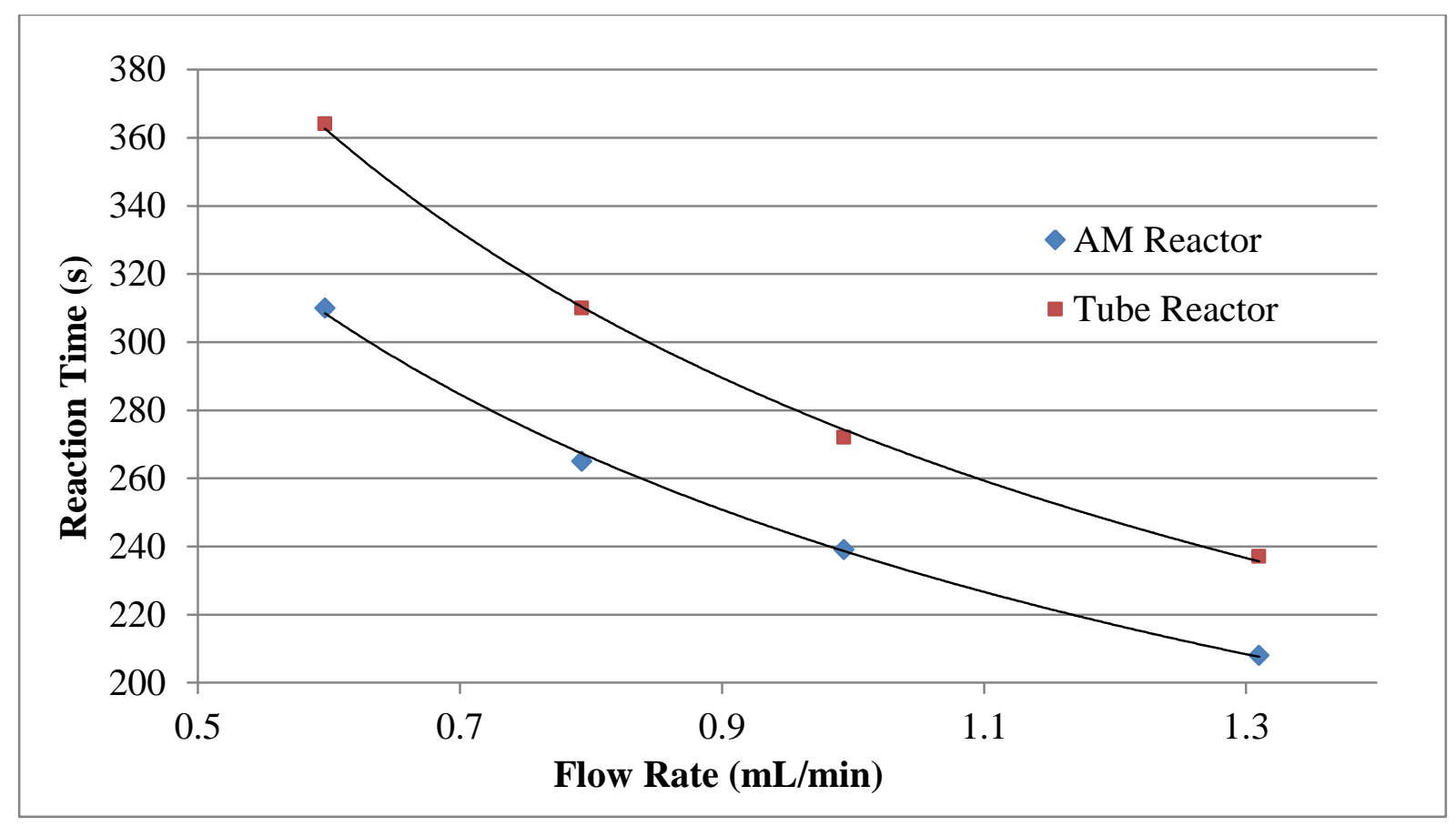

Figure 56: A graph to show reaction time versus flow rate for two different reactor systems. Reaction time is defined as the time taken for the reaction medium to change to the characteristic dark blue colour of the elemental iodine.

The intricate design of SL part 4 is certainly not achievable via conventional manufacturing processes. The design demonstrates the huge flexibility of AM, in terms of manufacturing intricate channel networks and complex mixing designs. There is huge scope in terms of designing and optimising channel networks to develop efficient mixer designs, which would not be manufacturable via subtractive manufacturing techniques. This work is unfortunately another research project in itself, and maybe an area where future research will be conducted.

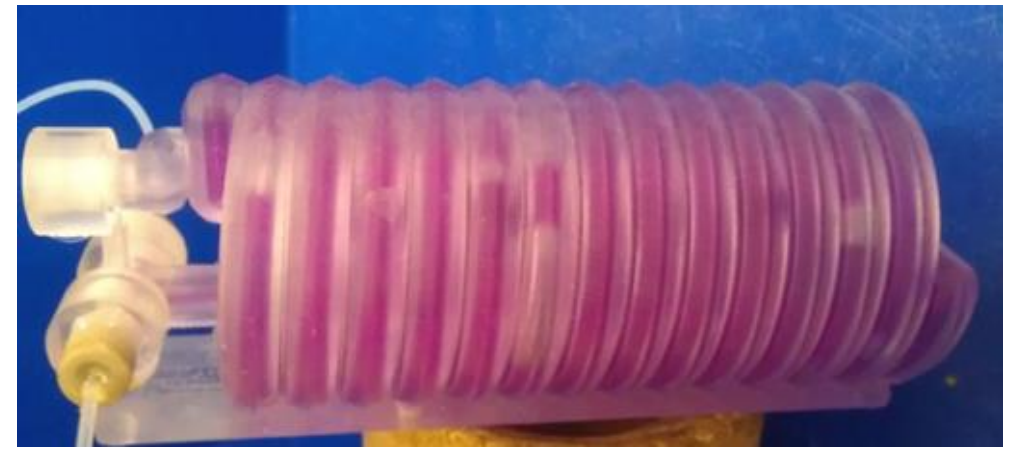

Figure 57: SL part 4 with a coloured solution being pumped through, confirming the sealing of the part.

Measurements of the part were taken in the $\mathrm{x}, \mathrm{y}$ and $\mathrm{z}$ plane, comparing this data with the original CAD to determine dimensional accuracy. 


\begin{tabular}{|c|c|c|c|c|}
\hline Dimension & $\begin{array}{c}\text { Actual Measurement } \\
(\mathbf{m m})\end{array}$ & $\begin{array}{c}\text { CAD measurement } \\
(\mathbf{m m})\end{array}$ & $\begin{array}{c}\text { Difference (+/- } \\
\mathbf{m m})\end{array}$ & $\begin{array}{c}\text { Variation } \\
(\boldsymbol{\%})\end{array}$ \\
\hline $\mathrm{x}$ & 27.55 & 27.50 & 0.05 & 0.18 \\
\hline $\mathrm{y}$ & 124.90 & 125.00 & 0.10 & 0.08 \\
\hline $\mathrm{z}$ & 50.59 & 50.50 & 0.09 & 0.18 \\
\hline
\end{tabular}

Table 26: Optical Calliper Measurements of SL Part 4.

\subsubsection{SL Part 5}

\section{Design, build and post processing:}

To this point, all the SL parts that had been produced had internal channel dimensions between 2 and $3 \mathrm{~mm}$ in diameter. Comparatively, most commercially available flow chemistry equipment will use standard 0.5 or $1 \mathrm{~mm}$ internal dimension tubing. In order to demonstrate that SL could be used to manufacture parts within this dimensional range, a series of short channels were manufactured within the same part, each with varying channel diameters (Figure 58). The channel dimensions are outlined in Table 27.

\begin{tabular}{|c|c|c|c|c|c|}
\hline Part Length (mm) & \multicolumn{5}{|c|}{55.00} \\
\hline Part Width (mm) & \multicolumn{5}{|c|}{9.00} \\
\hline Part Height (mm) & \multicolumn{5}{|c|}{23.00} \\
\hline Part Volume $\left(\mathrm{cm}^{3}\right)$ & \multicolumn{5}{|c|}{2.20} \\
\hline Channel Diameter (mm) & 0.50 & 0.75 & 1.00 & 1.25 & 1.50 \\
\hline Channel Length (mm) & 68.50 & 68.50 & 68.50 & 68.50 & 68.50 \\
\hline Internal Reaction Volume $\left(\mathrm{cm}^{3}\right)$ & 0.01 & 0.03 & 0.05 & 0.08 & 0.12 \\
\hline
\end{tabular}

Table 27: SL Part 5 Build Dimensions. 
Each of the tubes was built successfully and the un-cured resin was successfully removed from even the smallest channel.

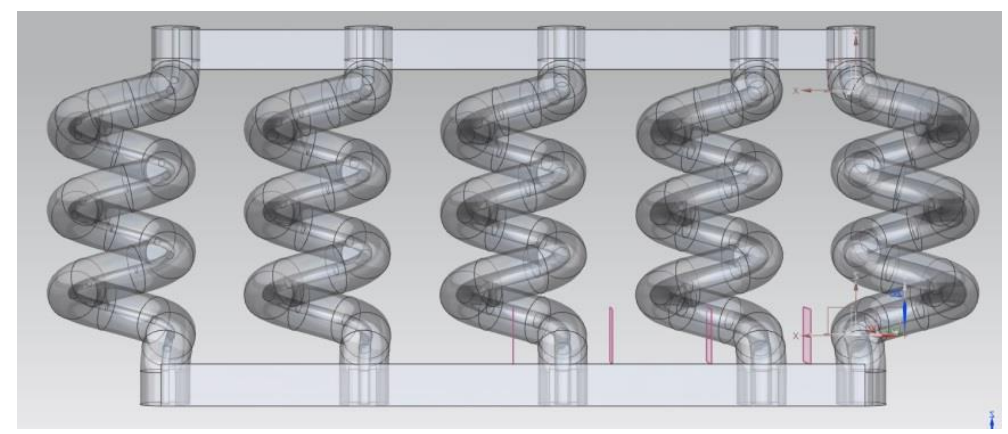

Figure 58: CAD model of SL part 5.

\section{Application/Discussion:}

SL part 5 demonstrated that it was possible to manufacture and clean channels with dimensions comparable to conventional tubing, as well as larger microfluidic devices. It still remained to be seen whether this scale of channel dimension could be incorporated into a more complex channel network design.

Measurements of the part were taken in the $\mathrm{x}, \mathrm{y}$ and $\mathrm{z}$ plane, comparing this data with the original CAD to determine dimensional accuracy.

\begin{tabular}{|c|c|c|c|c|}
\hline Dimension & $\begin{array}{c}\text { Actual Measurement } \\
(\mathbf{m m})\end{array}$ & $\begin{array}{c}\text { CAD measurement } \\
(\mathbf{m m})\end{array}$ & $\begin{array}{c}\text { Difference (+/- } \\
\mathbf{m m})\end{array}$ & $\begin{array}{c}\text { Variation } \\
\mathbf{( \% )}\end{array}$ \\
\hline $\mathrm{x}$ & 3.02 & 3.00 & 0.02 & 0.67 \\
\hline $\mathrm{y}$ & 8.96 & 9.0 & 0.04 & 0.44 \\
\hline $\mathrm{z}$ & 22.98 & 23.0 & 0.02 & 0.09 \\
\hline
\end{tabular}

Table 28: Optical Calliper Measurements of SL part 5.

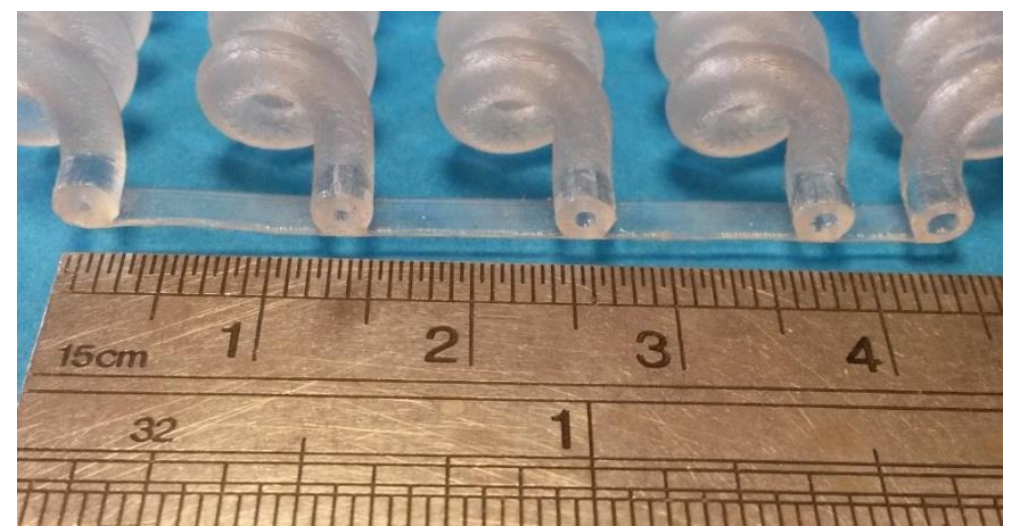

Figure 59: SL Part 5. 


\subsubsection{SL Part 6}

\section{Design, build and post processing:}

The next stage of our SL part development was to manufacture a part of sub-millimetre internal channel dimensions, whilst incorporating an intricate and convoluted channel network into the design. This part should have dimensions comparable to commercial microfluidic chips (Figure 60), preferably with channel widths less than $500 \mu \mathrm{m}$ in diameter. This will confirm that SL is capable of manufacturing parts that conform to existing microfluidic channel specifications.

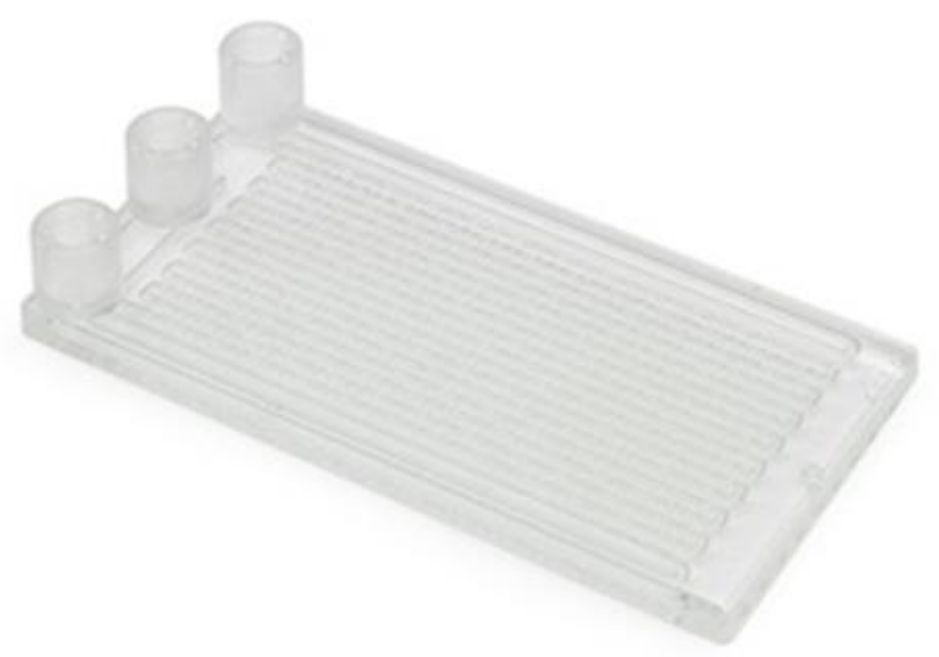

Figure 60: FlowSyn 2 mL static mixer chip.

Typically these microfluidic chips are manufactured from glass or PDMS, with the price of the $2 \mathrm{~mL}$ glass FlowSyn chip shown retailing at around £2400. To manufacture this type of bespoke part, glass etching manufacturing processes can be highly labour intensive, leading to the increased prices seen. ${ }^{45}$ AM may therefore prove to be a cost and time efficient method of manufacturing this type of complex micromixer. The design of the part would once again incorporate a SAR type mixer, as well as also incorporating a repeating, offset triangular pattern to the channel design. The pattern would hopefully induce even more efficient mixing within the channels. The part was built with the dimensions outlined in Table 29. 


\begin{tabular}{|c|c|}
\hline Part Length $(\mathrm{mm})$ & 75.00 \\
\hline Part Width $(\mathrm{mm})$ & 67.00 \\
\hline Part Height $(\mathrm{mm})$ & 19.70 \\
\hline Part Volume $\left(\mathrm{cm}^{3}\right)$ & 92.60 \\
\hline Channel Cross Sectional Area $\left(\mathrm{mm}^{2}\right)$ & 0.78 \\
\hline Channel Length $(\mathrm{mm})$ & 3380 \\
\hline Internal Reaction Volume $\left(\mathrm{cm}^{3}\right)$ & 2.65 \\
\hline
\end{tabular}

Table 29: SL Part 6 Build Dimensions.

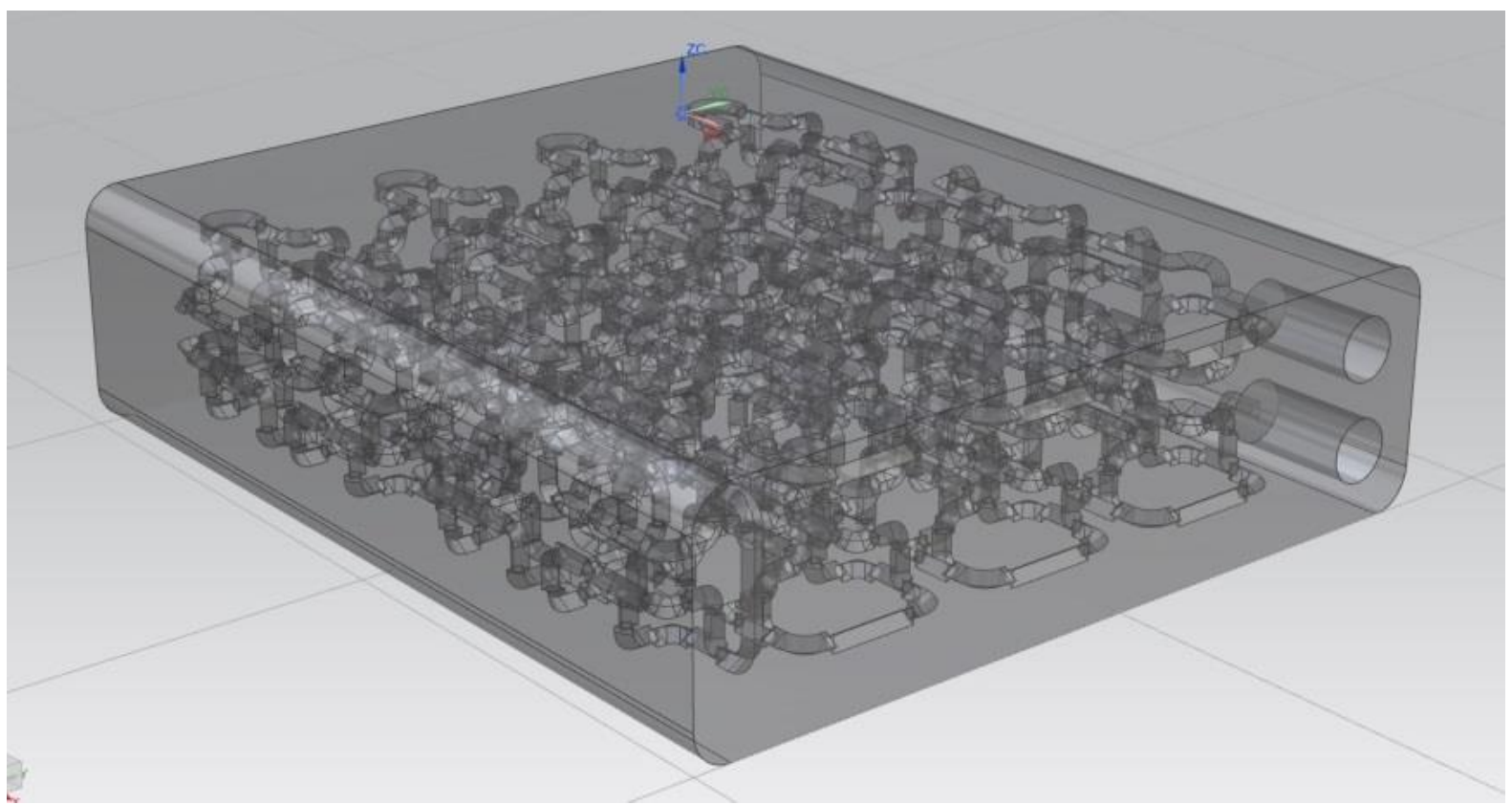

Figure 61: CAD model of SL part 6.

\section{Application/Discussion:}

Due to the length and small cross sectional area of the channel resin removal became almost impossible. The channel was eventually cleared after several hours of using a compressed air line. Unfortunately, when the channel was subjected to methanol solvent cleaning the channels appeared to become blocked after about 15 minutes of exposure. The hypothesis for this was that the swelling typically associated with the Accura 60 material on solvent exposure, caused the channels to swell shut. The cross-sectional intersect between the triangular sections of this build had an area equivalent to a $0.8 \mathrm{~mm}$ circular channel. This may mean that this material and manufacturing process are inappropriate for the manufacture of long convoluted channel networks with micron sized features, however more work is clearly required to confirm this hypothesis. 


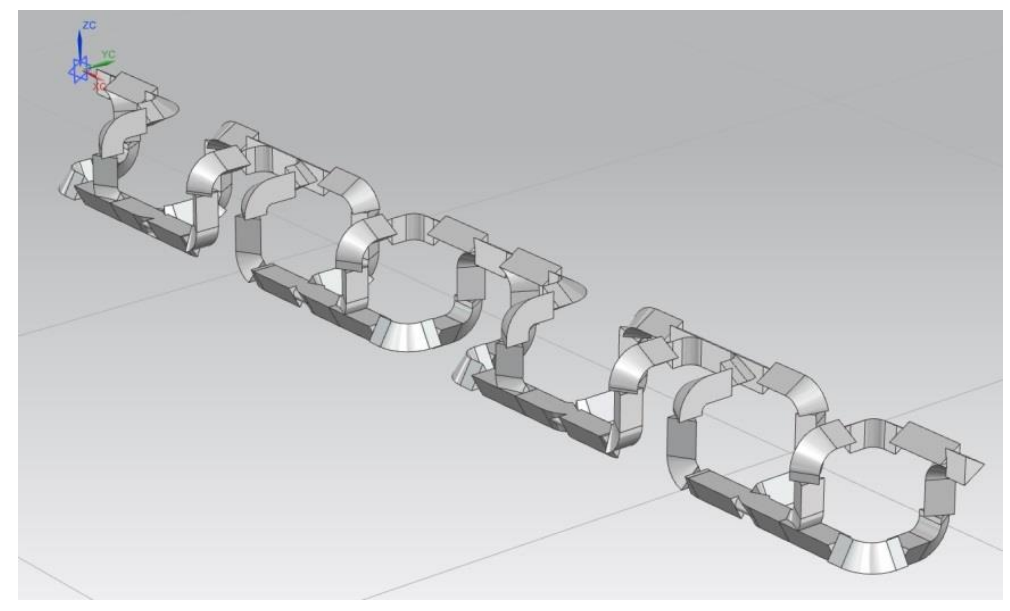

Figure 62: Repeating triangular pattern of SL part 6.

Measurements of the part were taken in the $\mathrm{x}, \mathrm{y}$ and $\mathrm{z}$ plane, comparing this data with the original CAD to determine dimensional accuracy.

\begin{tabular}{|c|c|c|c|c|}
\hline Dimension & $\begin{array}{c}\text { Actual Measurement } \\
(\mathbf{m m})\end{array}$ & $\begin{array}{c}\text { CAD measurement } \\
(\mathbf{m m})\end{array}$ & $\begin{array}{c}\text { Difference (+/- } \\
\mathbf{m m})\end{array}$ & $\begin{array}{c}\text { Variation } \\
(\%)\end{array}$ \\
\hline $\mathrm{x}$ & 75.03 & 75.0 & 0.03 & 0.04 \\
\hline $\mathrm{y}$ & 67.12 & 67.0 & 0.12 & 0.18 \\
\hline $\mathrm{z}$ & 19.75 & 19.7 & 0.05 & 0.25 \\
\hline
\end{tabular}

Table 30: Optical Calliper Measurements of SL Part 6.

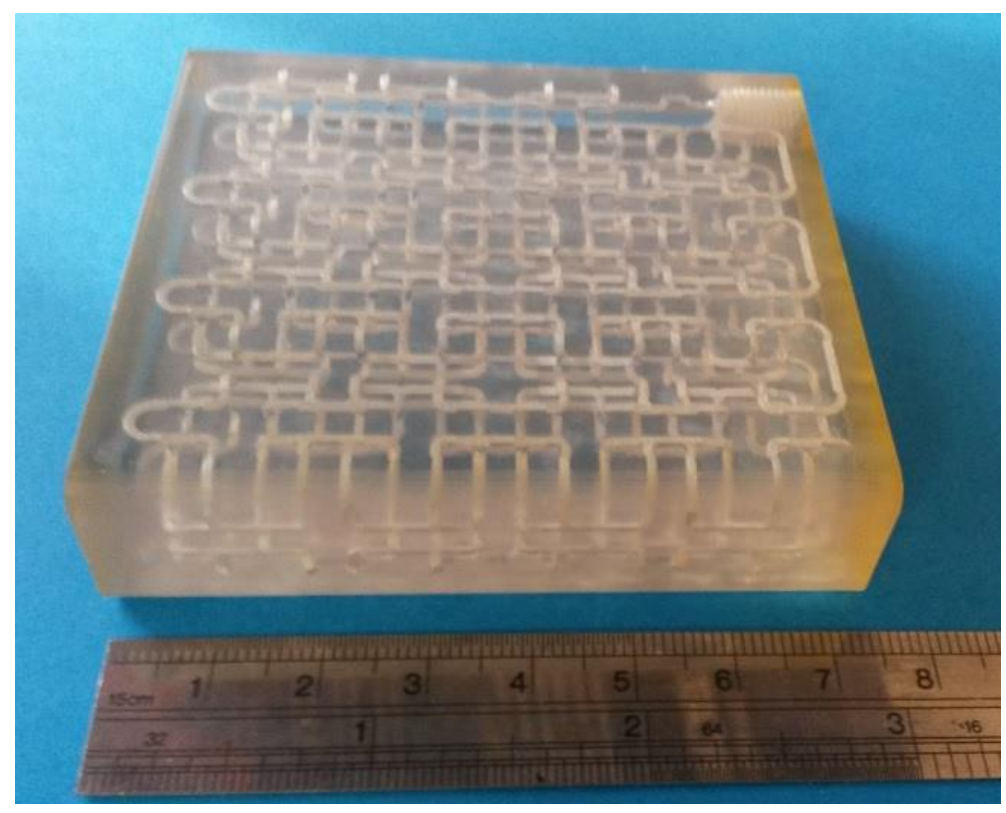

Figure 63: SL Part 6. 


\subsubsection{SL Part 7}

\section{Design, build and post processing:}

Collaborative work with University College London has seen the production of a chemical reactor for use in a continuous crystallisation process. These processes have been identified as a more efficient method of separating and purifying pharmaceutical compounds, than traditional batch crystallisation processes. ${ }^{149}$ The reactor design was compartmentalised into three temperature controlled sections. Each section of the part has channel networks above and below the reactor flow path, which are designed to have heated and cooled fluids pumped through. This will allow precise control over the reactor temperature during the crystallisation process. This type of temperature controlled reactor could have a huge number of possible flow chemistry applications, such as controlling localised exotherms or thermodynamic reaction optimisation. The fact that each part is separated means that thermal leaching between sections will be minimised. Alongside each turn of the channel network there is also a $0.5 \mathrm{~mm}$ channel inbuilt, into which a thermocouple could be placed. These thermocouples are designed to monitor the temperature of the reaction medium as accurately as possible as it passes through the channel.

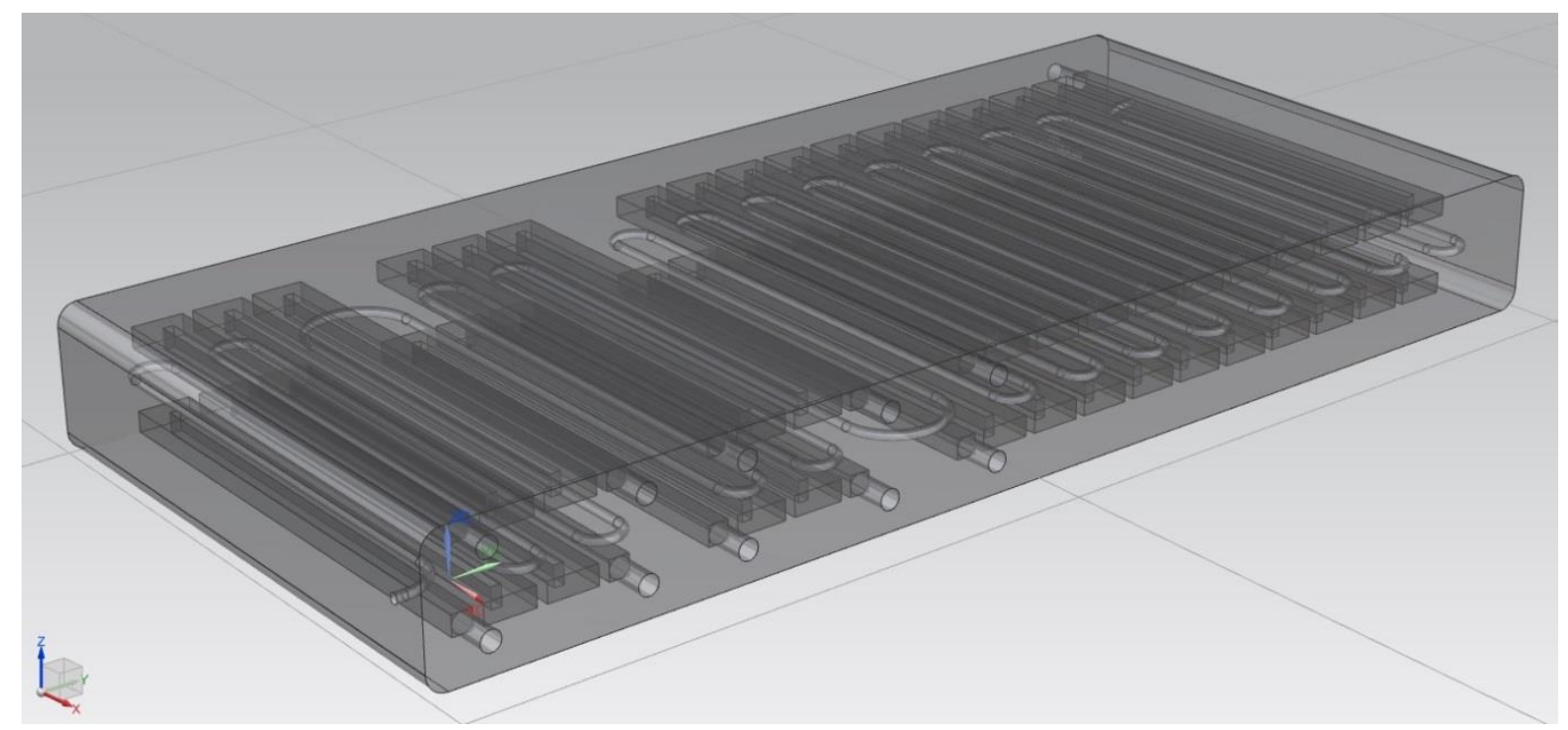

Figure 64: CAD model of SL part 7.

The part was built with the dimensions outlined in (Table 31). 


\begin{tabular}{|c|c|}
\hline Part Length $(\mathrm{mm})$ & 153.0 \\
\hline Part Width $(\mathrm{mm})$ & 58.0 \\
\hline Part Height $(\mathrm{mm})$ & 14.0 \\
\hline Part Volume $\left(\mathrm{cm}^{3}\right)$ & 107.3 \\
\hline Channel Diameter $(\mathrm{mm})$ & 1.00 \\
\hline Channel Length $(\mathrm{mm})$ & 1650 \\
\hline Internal Reaction Volume $\left(\mathrm{cm}^{3}\right)$ & 1.35 \\
\hline
\end{tabular}

Table 31: SL Part 7 Build Dimensions.

\section{Application/Discussion:}

The part was utilised by our collaborators at UCL for a number of proof of concept continuous crystallisation processes. Unfortunately, during the process it was shown that the channels blocked very easily, even at low seed concentrations and under conditions in which no crystal growth was expected. The seeds were shown to be settling and forming encrustations on the walls which lead to clogging. Clogging was delayed as the seed solution flow rate was increased, however at these high flow rates the crystals did not have enough time to grow within the channel. In order to overcome this issue the group tried to create droplets of the seed solution in an immiscible carrier fluid so as to avoid contact between the seed crystals and the walls. However, these experiments were also unsuccessful as the droplets generated were not stable within the reactor.
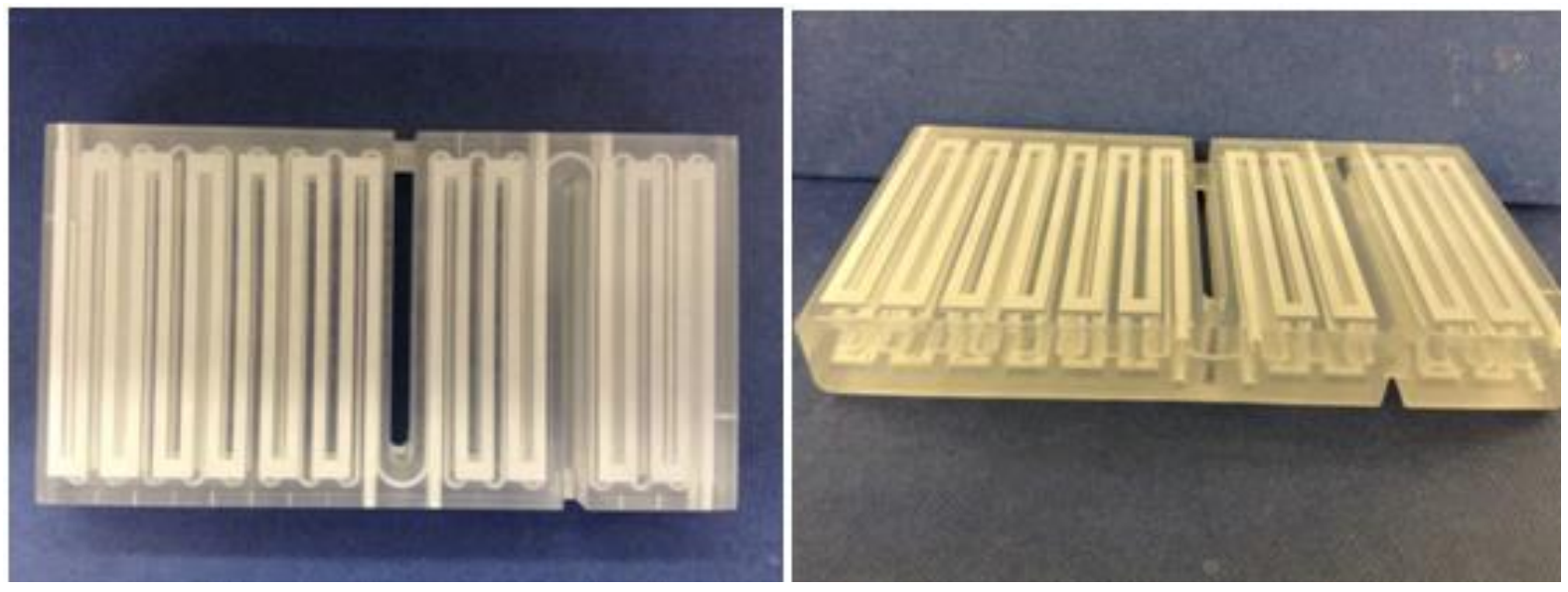

Figure 65: SL reactor 7.

The group speculated that the cause of the disruption of the stable droplets created outside the channel was due to disruption of the flow due to non-perfect alignment between the tubing and inlet boundary. Non perfect alignment was very likely since the connections were glued 
into the chip. Unfortunately, the temperature control channels above and below the crystallisation channel made visualisation of the part complex, making it difficult to accurately ascertain the exact site of the disruption. Although this section of work has yet to reach its conclusion, it still demonstrates the possibilities of using AM to manufacture parts with multiple flow paths, inbuilt sensors and complex internal geometries. This was all achieved with a single time and cost efficient manufacturing step. Once again the production of this type of part would be costly and time consuming using conventional glass etching processes.

\subsubsection{SL Part 8}

\section{Design, build and post processing:}

In order to overcome these visualisation problems, the temperature control channels were removed, as well as tapping each inlet and outlet to allow the connection of commercially available PEEK fittings. The part was built with the dimensions outlined in Table 32.

\begin{tabular}{|c|c|}
\hline Part Length $(\mathrm{mm})$ & 153.0 \\
\hline Part Width $(\mathrm{mm})$ & 58 \\
\hline Part Height $(\mathrm{mm})$ & 14 \\
\hline Part Volume $\left(\mathrm{cm}^{3}\right)$ & 95.9 \\
\hline Channel Diameter $(\mathrm{mm})$ & 1 \\
\hline Channel Length $(\mathrm{mm})$ & 1650 \\
\hline Internal Reaction Volume $\left(\mathrm{cm}^{3}\right)$ & 1.35 \\
\hline
\end{tabular}

Table 32: SL Part 8 Build Dimensions. 


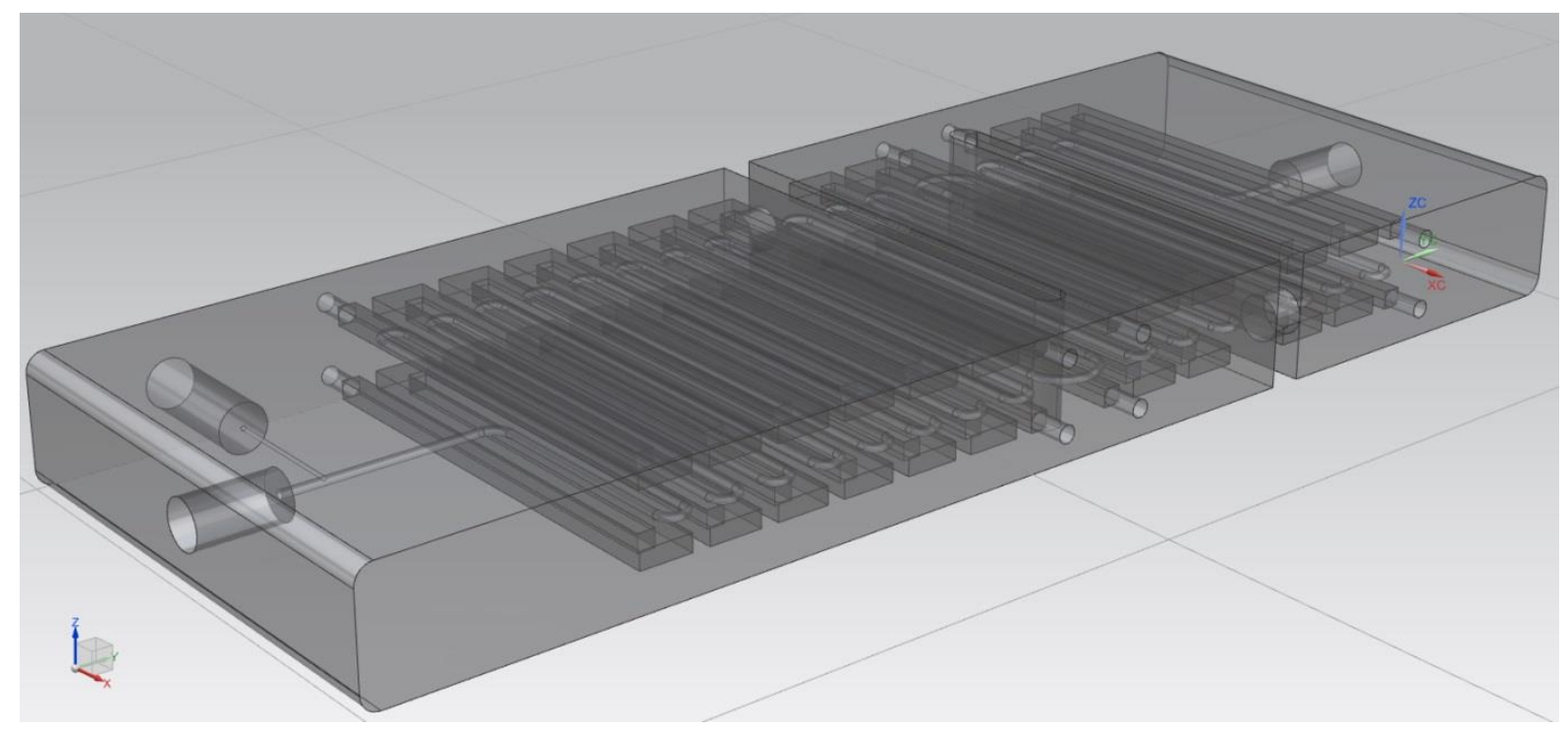

Figure 66:CAD model for SL reactor 8.

\section{Application/Discussion:}

Although this part has yet to be trialled, through this work, valuable insight can be gained into not only millifluidic reactor design, but also how the unique design freedom associated with AM can be utilised to induce precise temperature control within our reactions. It is also an aim to increase knowledge into how we can incorporate sensors/analytical devices into AM chemical reactors, such as the thermocouples embedded into this device.

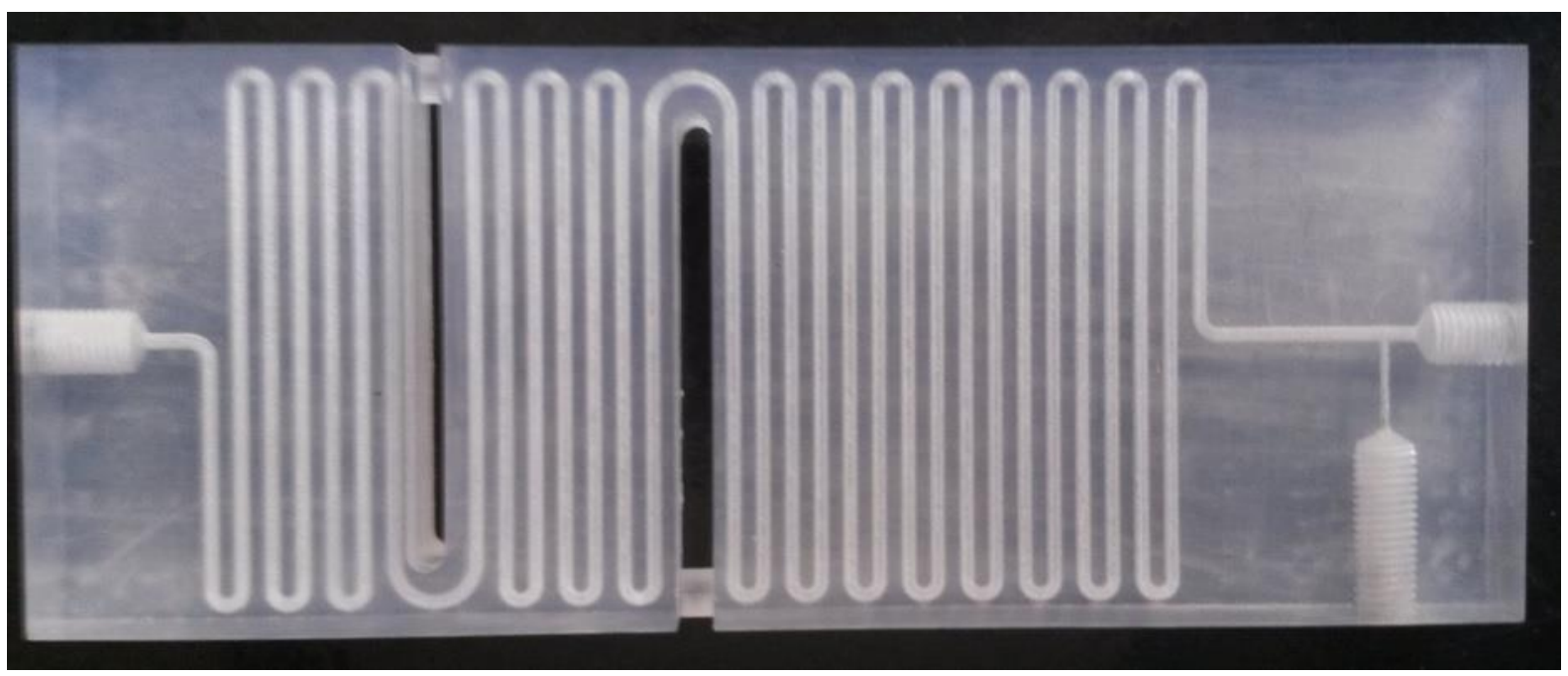

Figure 67: SL reactor 8.

A number of other SL parts have been built during the course of this research, however they are discussed in more detail during chapter 7.2. 
In general, dimensional accuracy of parts built from Accura 60 via the SL process using a Viper SL system was excellent. The six parts built and measured during this chapter were all within $+/-120 \mu \mathrm{m}$ of the intended dimensions, with parts on average being within $+/-44 \mu \mathrm{m}$ of the intended geometry. This on average is $0.14 \%$ from the true value.

\subsection{Conclusion}

Undoubtedly, SL has considerable potential when manufacturing milli and even micro-scale fluidic devices. The wide range of bespoke customisable geometries that can be manufactured, as well as build resolutions comparable to existing subtractive manufacturing processes, demonstrates the flexibility of SL as a suitable manufacturing process for this application. Unfortunately, the limited number of resins commercially available for SL processing is often cited as the limiting factor of SL as a manufacturing process. Current commercially available SL materials have poor thermal and chemical compatibility, as demonstrated in our own experiments, as well as often being glassy, rigid and highly brittle. This brittleness can often lead to issues associated with post-processing of the parts i.e., application of threaded channels. These properties often prohibit the use of SL for synthetic flow applications, although future material development may well overcome these hurdles. Indeed the initial research conducted here has highlighted some important issues regarding materials properties, particularly machinability of the resin and long term degradation of parts which are exposed to different environmental conditions. SL is rapidly becoming a cost efficient, user friendly and highly customisable method of fabricating intricate channel networks. With the advent of desktop and home SL systems leading to dramatic reductions in equipment and materials costs, the drive to develop new ranges of SL materials may well lead to this manufacturing process replacing existing soft lithographic manufacturing processes.

\section{4. $\quad$ Future Work}

During the course of this research, we have demonstrated that a key advantage of manufacturing using SL is the design freedom and geometrical accuracy possible. Future research within this group will therefore focus on how this design freedom can be used to incorporate added functionality into our channel networks. Some initial research in later chapters details how quartz windows can be embedded into AM parts for spectroscopic 
analysis, however there still remains a wide array of sensors and analytical devices which can be incorporated into reagent streams to allow inline reaction analysis. This type of functionalised device would not only afford more precise control over their reaction conditions, but would do so in a way that is ordinarily either highly expensive or impossible to achieve via conventional manufacturing processes. 


\section{6. $\quad$ Selective Laser Melting}

\subsection{Introduction}

Selective laser melting (SLM) is a solid freeform AM process whereby highly complex threedimensional parts are fabricated in a layer-by-layer process. SLM can manufacture parts in a range of metal powders such as stainless steel, aluminium, titanium, etc., and is therefore an attractive technique for a number of industrial applications ranging from aerospace to Formula $1 .^{150}$ The SLM process typically occurs via the sequential scanning of a metal powder bed with a high powered laser, ${ }^{151}$ although other more novel materials have been demonstrated. ${ }^{152}$ The build process again begins with the generation of a 3D CAD model of the build part, which is generally sliced sequentially into layers of around 20-150 $\mu \mathrm{m}$. These sections are built one at a time in an inert atmosphere, heated build chamber. A high powered laser beam is used to sequentially scan each layer of powder, marking out the predefined pattern of each layer. The powder delivery piston raises a set distance as each metal layer is melted, with a roller or wiper spreading a programmed thickness of metal powder across the build chamber. This layer is carefully levelled by a soft wiper blade to ensure an even distribution of powder across the build plate. The process is repeated until each section has been scanned and the part has been built. ${ }^{153}$ When the build is complete the chamber is allowed to cool to ambient temperature, then the part is removed from the build chamber, cleaned and any undesired support structures are removed. ${ }^{154}$ These structures are put into place to support any overhanging features of the build, and to prevent the collapse of the molten metal during the build process. They are made from the same metal powder as the build itself.

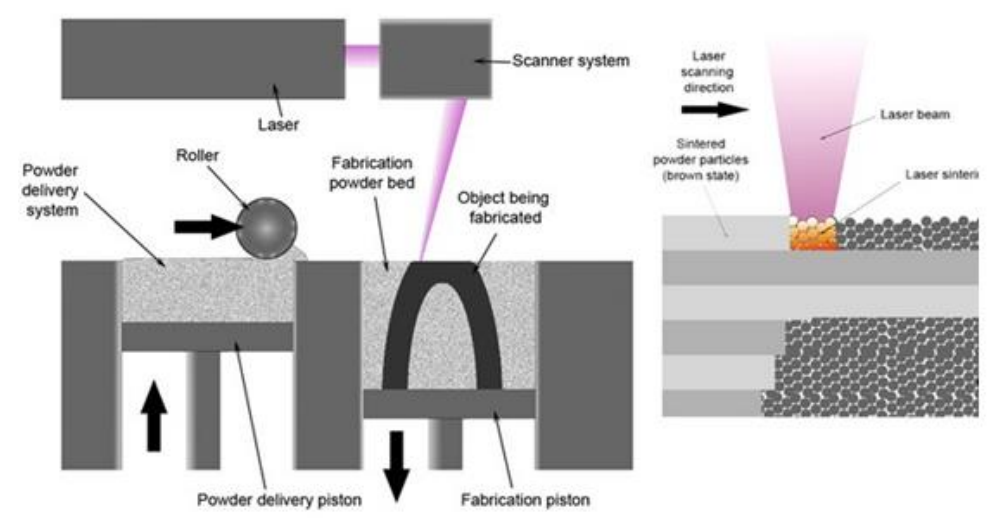

Figure 68: The SLM build process. ${ }^{155}$ 
Due to the high concentration of energy around the laser build spot, SLM can produce fully consolidated parts with minimal porosity, and mechanical properties comparable to bulk metals. Re-melting between layers, and the lower viscosity of liquid metal compared to polymers, ensures fully dense parts and relatively isotropic physical properties. SLM machines are available from a number of commercial manufacturers and in a variety of sizes. However, they generally utilise a fibre laser of 100 to $400 \mathrm{~W}$ and build in comparatively thin layers, typically 0.02 to $0.1 \mathrm{~mm}$. It is common to recycle unused powder which is sieved to remove oversized particles. Powders may also be dried to improve flow during the build process. Unlike thermoplastic laser sintering (LS), unused powder remains unaffected and can be re-used. Dimensional accuracy is highly geometry dependent, and potentially affected by heat distortion as well as inherent machine characteristics. Parameters such as the size of the laser spot, laser power, hatch distance (the distance between consecutive scan lines), layer thickness scan pattern and the scanning velocity can be varied, allowing the inputted energy to be accurately controlled to facilitate more accurate builds. In general, parts are built at a rate of around $5-20 \mathrm{~cm}^{3} / \mathrm{h}$, depending on individual build parameters, ${ }^{154}$ with accuracies of +/- $0.05 \mathrm{~mm}$ being achieved over smaller parts. Laser spot size variations between 0.3 and 0.7 $\mathrm{mm}$ did however dictate the minimum wall thickness achievable during our research. Repeatability is generally good once a particular part has been optimised for the build process. SLM manufacturing is designed to produce near-full-density parts, however porosity can be a feature of SLM parts; ${ }^{156}$ due to the large energy input of the laser beam, problems such as balling (a phenomenon seen when the molten material adheres to the underlying substrate due to the surface tension) can occur which can result in a rough particle surface which obstructs a smooth layer deposition and decreases the density of the produced part. Other common inaccuracies in SLM manufactured parts are caused by residual stressing of parts which can lead to deformation. These stresses can often lead to manufacturing failures due to de-lamination, distortion and cracking. ${ }^{157}$

SLM offers a range of highly chemically and thermally stable materials with which to manufacture flow reactors. The parts produced via SLM have the capability to create an array of functional components comparable to commercially available micro and millifluidic devices. Highly stable oxide layers make metals such as stainless steel, aluminium and titanium resistant to almost all organic reaction media, with concentrated acid solutions often being required to remove the oxide layer. These metals also have extremely good thermal conductivities, with most parts being stable well beyond the temperature ranges typically 
employed during most flow processes. A wide range of specialist alloys such as Inconel® (a nickel-chromium alloy) are also available, offering resistance to extreme temperature and pressures.

During the course of this research metal parts were manufactured using a Renishaw AM250, Realizer SLM 100 or a Realizer SLM 50. The Renishaw AM250 is the largest of the 3 machines, with a build platform of 250 x 250 x $360 \mathrm{~mm}$ (x,y,z). Scan speeds of up to 2000 $\mathrm{mm} / \mathrm{s}$ allow parts to be built at rates of as much as $20 \mathrm{~cm}^{3} / \mathrm{h}$, depending on the part geometry and build parameters. The laser power can be set to 200 or $400 \mathrm{~W}$, allowing fully dense parts to be realised with defined layer thicknesses of $20 \mu \mathrm{m}$. The laser beam diameter is variable, going as low as $70 \mu \mathrm{m}$ at $200 \mathrm{~W}$ laser power, and $135 \mu \mathrm{m}$ at $400 \mathrm{~W}$. The AM250 was used to produce larger build parts, which do not require intricate channel designs. The Realizer SLM 100 has a smaller build platform of 125 x 125 x 75 mm (x,y,z). Build speeds are typically 5$30 \mathrm{~cm}^{3} / \mathrm{h}$, depending on the part geometry and build parameters. The laser power can be set to between 20 and $200 \mathrm{~W}$, allowing fully dense parts to be realised with defined layer thicknesses of 20-100 $\mu \mathrm{m}$. The laser beam diameter can range between 30 and $100 \mu \mathrm{m}$. The smaller, more precise laser used by the SLM 100 makes this machine ideal for smaller parts, with more complex internal geometries. The Realizer SLM 50 is the smallest of the three machines, with a circular build platform of diameter $70 \mathrm{~mm}$, and an achievable part height of $40 \mathrm{~mm}$. The laser power can be set to between 20 and $120 \mathrm{~W}$, allowing parts to be realised with defined layer thicknesses of $20-50 \mu \mathrm{m}$. The laser beam diameter can be set to $10 \mu \mathrm{m}$. The SLM 50 will be ideal for manufacturing the smallest of parts, which require highly accurate design features.

These machines are capable of processing a range of materials to a high geometrical resolution. The processable materials include stainless steel 316L and 17-4PH, H13 tool steel, aluminium $\mathrm{Al}-\mathrm{Si}-12$ and $\mathrm{Al}-\mathrm{Si}-10$, titanium $\mathrm{CP}$, Ti-6Al-4V and Ti-6Al-Nb, cobalt-chrome and Inconel 718 and 625. Although a range of other materials have been processed, it was opted to only utilise the materials previously listed, due to the geometrically accurate and reproducible quality of parts which they produce. Of those materials, during the course of this research parts were manufactured using $316 \mathrm{~L}$ stainless steel and Ti-6Al-4V titanium. This was largely due to availability and cost of the materials, however both materials are well trusted within the flow chemistry community due to their high thermal and chemical stability. 


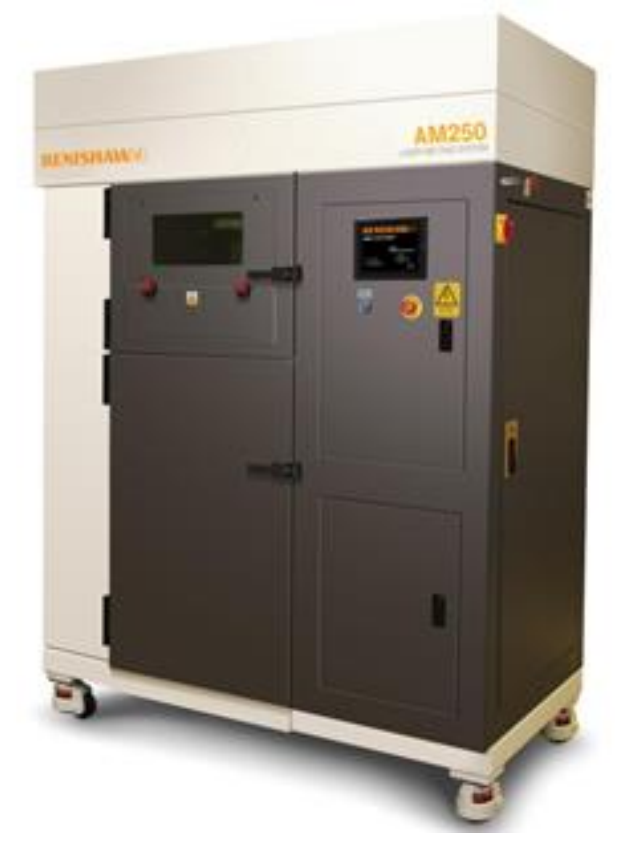

Figure 69: The Renishaw AM250 used during this research. ${ }^{158}$

\subsection{Results and Discussion}

\subsubsection{SLM Part 1}

\section{Design, build and post processing:}

SLM part 1 was designed as a proof of concept, to demonstrate the capability of SLM by building a simplistic channel network which was capable of flowing liquids through at elevated pressures. The reactor (which mirrored SL part 1 in design) was built from Ti-6Al4V alloy using the Realizer SLM 100 with the dimensions outlined in Table 33.

\begin{tabular}{|c|c|}
\hline Part Length (mm) & 30.50 \\
\hline Part Width $(\mathrm{mm})$ & 30.50 \\
\hline Part Height $(\mathrm{mm})$ & 50 \\
\hline Part Volume $\left(\mathrm{cm}^{3}\right)$ & 45.13 \\
\hline Channel Diameter $(\mathrm{mm})$ & 2.00 \\
\hline Channel Length $(\mathrm{mm})$ & 439 \\
\hline Internal Reaction Volume $\left(\mathrm{cm}^{3}\right)$ & 1.38 \\
\hline
\end{tabular}

Table 33: SLM Part 1 Build Dimensions.

The part was removed from the build plate using an AgieCharmilles CUT 20P wire erosion system. Non-melted powder was removed from within the tubes using a compressed air line, 
and the reactor was tapped at each end using a 10-32 UNF screw thread, which proved more difficult than previous SL tapping due to it being manufactured from titanium alloy.

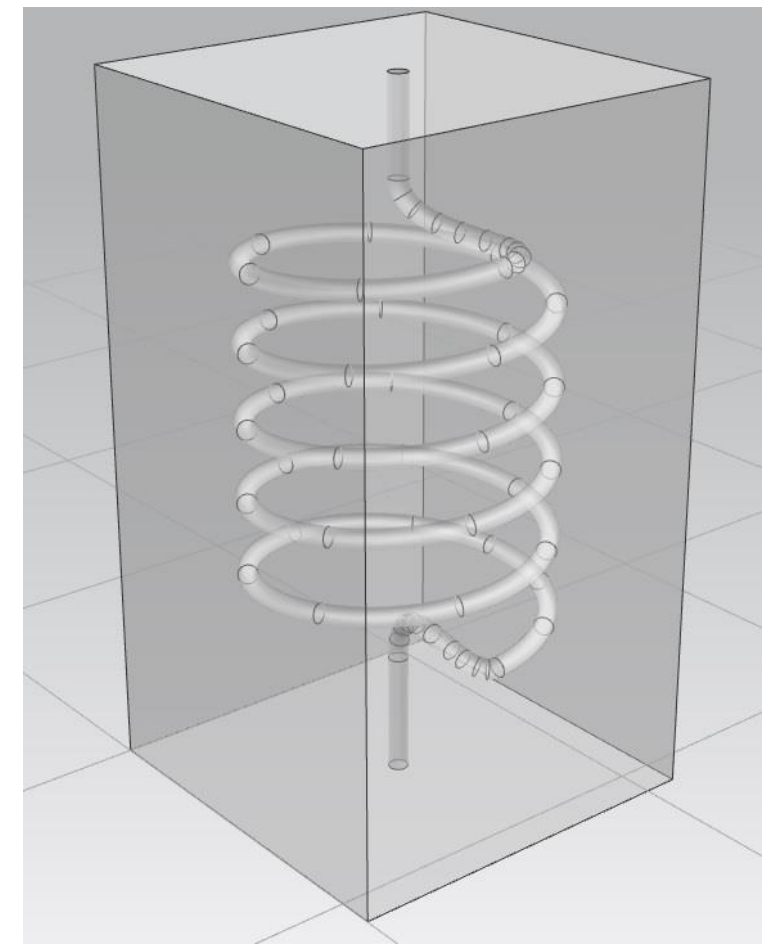

Figure 70: CAD model of SLM Part 1.

\section{Application/Discussion:}

Due to the relatively small internal reaction volume of the part, it was impractical to use it for any functional flow chemistry. In order to determine whether the part was capable of withstanding continuous flow processes at elevated pressures, it was attached to a commercially available FlowSyn, and a range of common laboratory solvents $(\mathrm{MeOH}$, acetone, DCM, water) were pumped through the part at pressures of up to 20 bar and temperatures up to $200^{\circ} \mathrm{C}$ for a period of $1 \mathrm{~h}$ per solvent. The part showed no visible sign of degradation, and there were also no issues with the part leaking at the elevated pressures being operating at. This is in contrast to the work previously done using SL, whereby the materials demonstrated swelling in a range of solvents, clearly demonstrating the advantage of using SLM to manufacture parts for this application.

Although SLM part 1 was not used for any flow chemistry, it did demonstrate the potential for SLM to manufacture functional flow reactors capable of withstanding the elevated pressures and temperatures of typical flow processes. It also demonstrated that it was possible 
to successfully clear these parts of non-melted metal powder, and attach them to commercial flow equipment via typical HPLC PEEK fittings.

Measurements of the part were taken in the $\mathrm{x}, \mathrm{y}$ and $\mathrm{z}$ plane, comparing this data with the original CAD to determine dimensional accuracy.

\begin{tabular}{|c|c|c|c|c|}
\hline Dimension & $\begin{array}{c}\text { Actual } \\
\text { Measurement }(\mathbf{m m})\end{array}$ & $\begin{array}{c}\text { CAD measurement } \\
(\mathbf{m m})\end{array}$ & $\begin{array}{c}\text { Difference } \\
(+/-\mathbf{m m})\end{array}$ & $\begin{array}{c}\text { Variation } \\
(\boldsymbol{\%})\end{array}$ \\
\hline $\mathrm{x}$ & 30.44 & 30.50 & 0.06 & 0.19 \\
\hline $\mathrm{y}$ & 30.39 & 30.50 & 0.11 & 0.36 \\
\hline $\mathrm{z}$ & 49.95 & 50.00 & 0.05 & 0.10 \\
\hline
\end{tabular}

Table 34: Optical Calliper Measurements of SLM Part 1.

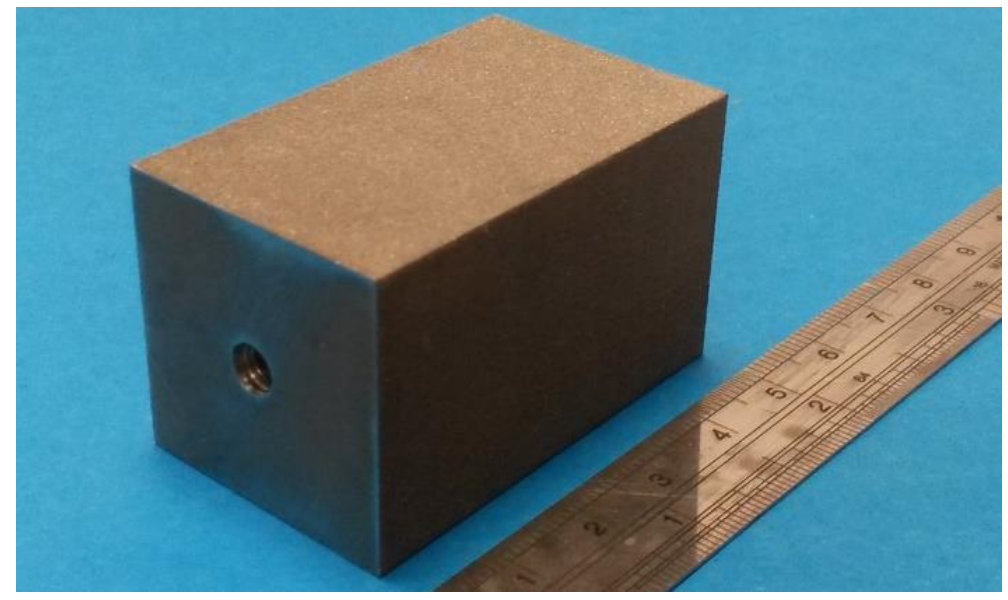

Figure 71: SLM Part 1.

A discussion about the accuracy and repeatability of parts built from via the SLM process will follow later in the chapter.

\subsubsection{SLM Part 2}

\section{Design, build and post processing:}

Having successfully demonstrated that it was possible to manufacture a simple channel via SLM, the next stage of the development would be to manufacture a part with sufficient internal reaction volume (and consequently residence time) to perform a wide range of chemical reactions. The reactor was again built from Ti-6Al-4V alloy using the Renishaw AM250. The AM250 was suited to building this part, due to the large total volume of the build and the lack of any detailed features. The internal channel geometry was again designed 
with a helical structure to facilitate powder removal. The part was built with the dimensions outlined in Table 35.

\begin{tabular}{|c|c|}
\hline Part Length $(\mathrm{mm})$ & 153.0 \\
\hline Part Width $(\mathrm{mm})$ & 58 \\
\hline Part Height $(\mathrm{mm})$ & 14 \\
\hline Part Volume $\left(\mathrm{cm}^{3}\right)$ & 95.9 \\
\hline Channel Diameter $(\mathrm{mm})$ & 2 \\
\hline Channel Length $(\mathrm{mm})$ & 6580 \\
\hline Internal Reaction Volume $\left(\mathrm{cm}^{3}\right)$ & 20.7 \\
\hline
\end{tabular}

Table 35: SLM Part 2 Build Dimensions.

The part was removed from the build plate and was tapped at each end using a 10-32 UNF screw thread.

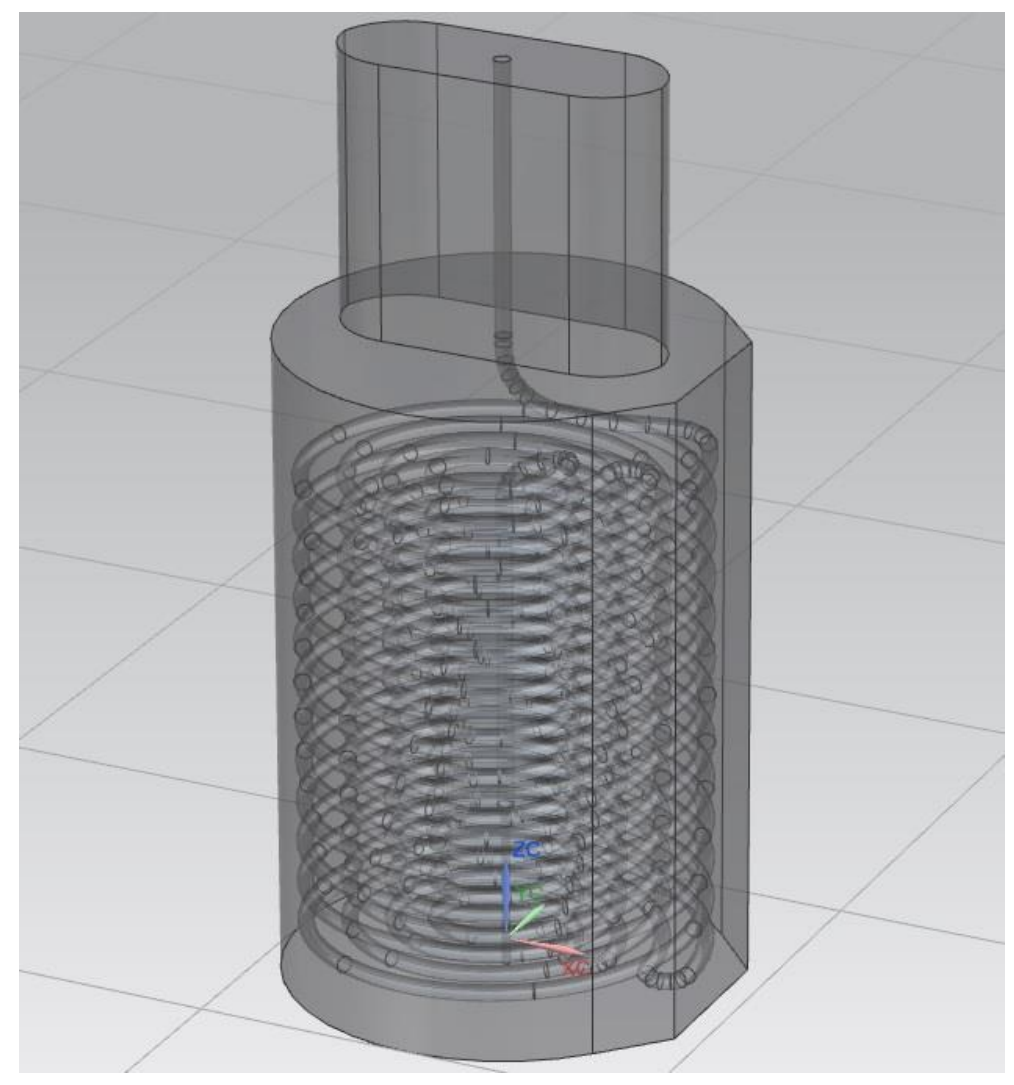

Figure 72: CAD model for SLM part 2.

\section{Application/Discussion:}

Although the build was successful, it proved difficult to remove the non-melted powder from the internal channels of the part. This was attempted by sonication, vacuum suction, agitation, 
heating and cooling, and application of a compressed air line, however all of these methods were unsuccessful at removing any powder from the build. A portion of the reactor was cut open to determine the cause of the blockages. It was found that some of the theoretically nonmelted powder had formed a semi-solid causing substantial blockages within the channel. Two theories were presented for the cause of the blockages. Firstly, the substantially increased length of the channel network meant that the pressure required to dislodge the powder would be significantly increased. The second theory was that, as a consequence of the high powered laser used in SLM, there would have been a large build up in energy and heat within the reactor during the build process. This build up in temperature may have caused partial sintering of the powder to the internal surfaces of the channels, potentially reducing the diameter of the channel and, maybe in extreme incidences, sealing them. As well as this, the expansion and subsequent contraction of the metal channels, caused by the considerable changes in temperature during the build process, may have led to the remaining non-melted powder being compressed into the semi-solid being observed. For this reason there is limited literature precedent for the manufacture of hollow parts, or with those with complex internal channel networks, via the SLM manufacturing process.

The outer surface of the part displayed the characteristic surface roughness currently associated with SLM manufacturing, however by visual inspection it seemed that this roughness was more pronounced in the internal structures. Visualisation of the roughness of these internal channels was achieved via optical microscopy, using a GX Microscope L2003B HTG. A $5 \mathrm{~mm}$ slice was taken from the end of the part, again using the AgieCharmilles CUT 20P wire erosion system. These images showed to us the considerable surface roughness of the channel, with large beads of melted powder extruding from the wall of the channel. These beads ranged in size from large agglomerations of powder particles down to smaller clusters of particles. As it stands these areas of surface roughness are an unwanted feature of using SLM, however it is possible that if this roughness could be controlled it could be utilised to induce turbulent mixing within the channel or to increase channel surface area for applications such as catalyst attachment. 


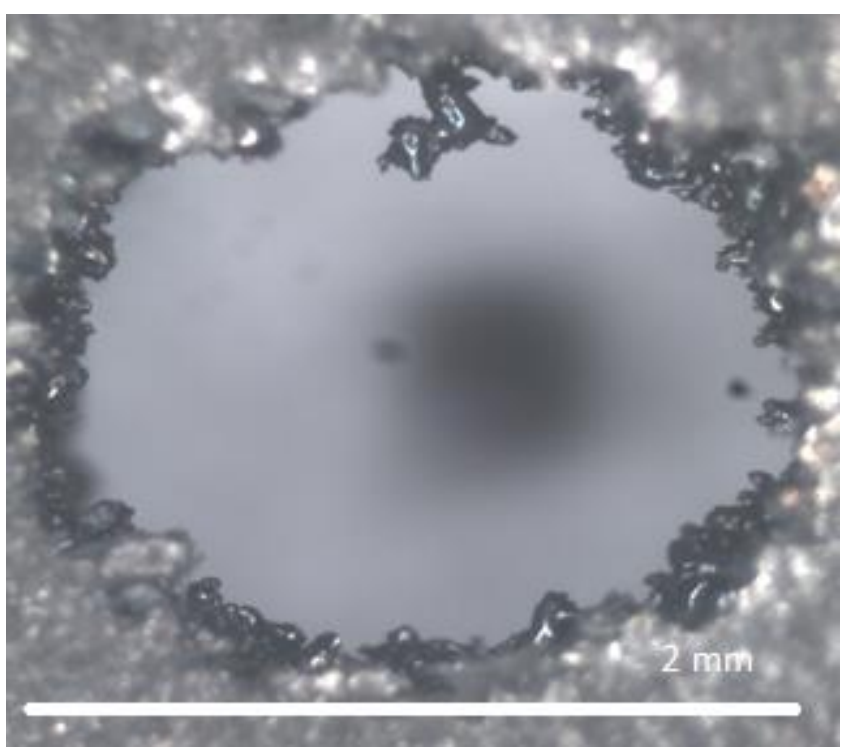

Figure 73: Cross section of SLM Part 1 visualised under a GX Microscope L2003B HTG microscope.

Measurements of the part were taken in the $\mathrm{x}, \mathrm{y}$ and $\mathrm{z}$ plane, comparing this data with the original CAD to determine dimensional accuracy.

\begin{tabular}{|c|c|c|c|c|}
\hline Dimension & $\begin{array}{c}\text { Actual } \\
\text { Measurement }(\mathbf{m m})\end{array}$ & $\begin{array}{c}\text { CAD measurement } \\
(\mathbf{m m})\end{array}$ & $\begin{array}{c}\text { Difference } \\
(+/-\mathbf{m m})\end{array}$ & $\begin{array}{c}\text { Variation } \\
(\boldsymbol{\%})\end{array}$ \\
\hline $\mathrm{x}$ & 20.11 & 20.00 & 0.11 & 0.55 \\
\hline $\mathrm{y}$ & 40.20 & 40.00 & 0.20 & 0.50 \\
\hline $\mathrm{z}$ & 54.46 & 54.50 & 0.04 & 0.07 \\
\hline
\end{tabular}

Table 36: Optical Calliper Measurements of SLM Part 2.

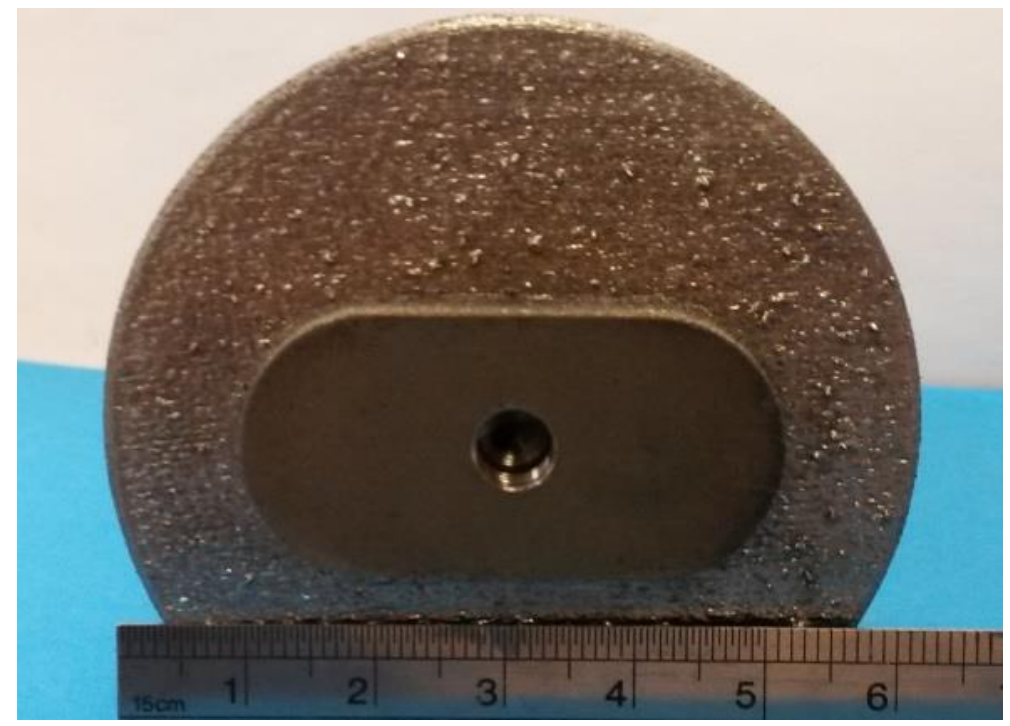

Figure 74: SLM Part 2. 


\subsubsection{SLM Part 3}

\section{Design, build and post processing:}

If our hypothesis that the channel surface roughness was causing difficulties with powder removal proved, to be correct it would be possible to facilitate powder removal via a number of different design strategies. A reduction in channel length, coupled with an increase in channel diameter, would lead to a reduction in the force required to clear the non-melted powder from the channels. Another option would be to remove the bulk mass of the part surrounding the channels, leaving what would be a rigid length of metal tubing. This would hopefully reduce the temperature build up within the part during the manufacturing process, reducing unwanted melting of powder within the channels. Ideally, our designs should have channel dimensions in the region of $0.5-1 \mathrm{~mm}$ in diameter, in order to mimic commercially available metal tubing, so we opted first to remove the bulk mass of the channel. A selection of smaller parts were manufactured from Ti-6Al-4V alloy, using the Renishaw AM250, trialling a range of channel lengths and internal channel diameters. These parts had the dimensions outlined in Table 37.

\begin{tabular}{|c|c|c|c|c|}
\hline Part & $\begin{array}{c}\text { Length } \\
(\mathbf{m m})\end{array}$ & $\begin{array}{c}\text { Internal Tube } \\
\text { Diameter }(\mathbf{m m})\end{array}$ & $\begin{array}{c}\text { External Tube } \\
\text { Diameter }(\mathbf{m m})\end{array}$ & $\begin{array}{c}\text { Internal Reaction } \\
\text { Volume }(\mathbf{m L})\end{array}$ \\
\hline $3 \mathrm{~A}$ & 170 & 1 & 5 & 0.13 \\
\hline $3 \mathrm{~B}$ & 170 & 2 & 6 & 0.53 \\
\hline $3 \mathrm{C}$ & 580 & 1 & 5 & 0.46 \\
\hline $3 \mathrm{D}$ & 580 & 2 & 6 & 1.82 \\
\hline
\end{tabular}

Table 37: Dimensions of SLM Parts 3. 


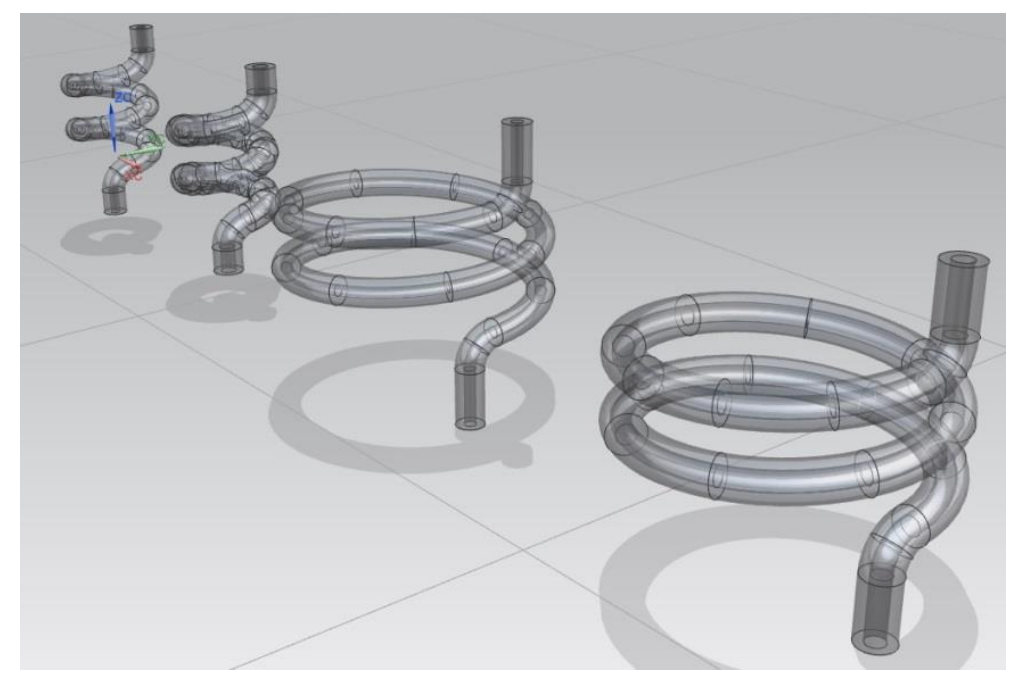

Figure 75: CAD drawings of SLM Parts 3. From right to left: 3D, 3C, 3B, 3A.

\section{Application/Discussion:}

Powder removal was successful for each of the 4 manufactured parts. The structures which were required to support the overhanging features of the design were left intact, once the part had been removed from the build plate. These structures would be very difficult to remove from this part without damaging the channel walls. This went some way to confirming our theory that the heat generated during the manufacture of parts, was causing unwanted partial melting of the powder within the channels. The next stage in this development would be to manufacture parts of similar dimensions but with a much larger channel length.

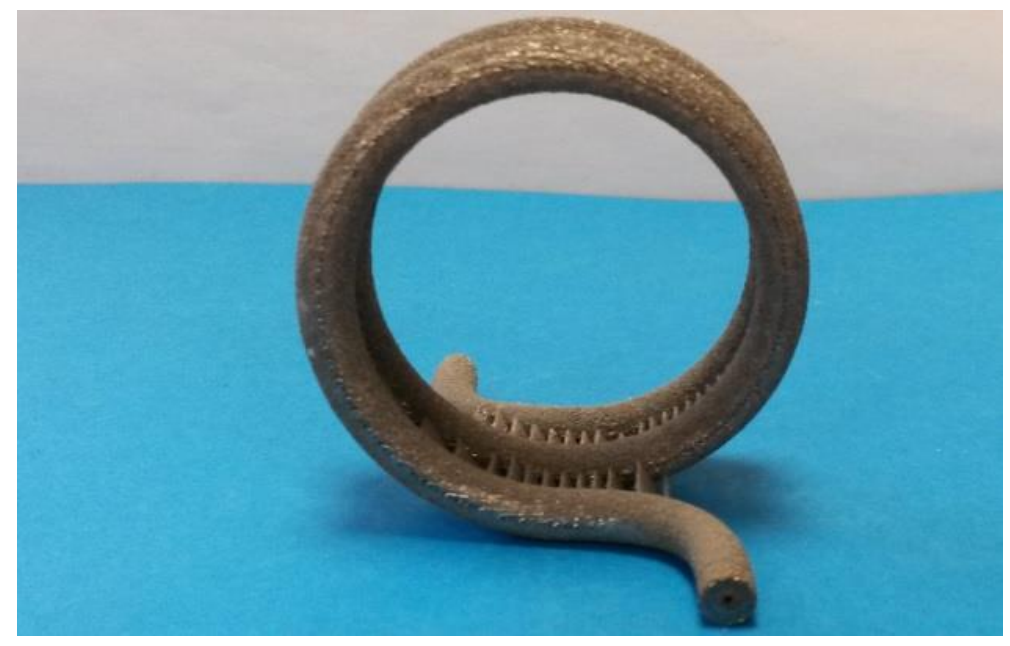

Figure 76: SLM Part 3D.

Upon visual inspection of our parts there was still considerable internal channel surface roughness. This roughness was visualised, using a LEO 440 scanning electron microscope (SEM). In order to do this, we removed two sections from part 3D using an angle grinder; the 
first part from the section of the channel manufactured perpendicular to the build plate or in the $\mathrm{z}$ plane (Figure 77), and the second part from the section of the channel manufactured parallel to the build plate or the $x-y$ plane (Figure 78). The first part to be visualised was the portion of channel manufactured in the $\mathrm{z}$ plane. It was initially noticeable that the mass of metal remaining around the channel was undoubtedly a non-porous solid mass of metal. This should allow us to operate at the elevated pressures typically associated with flow chemistry. It was also clear that the internal surfaces of these channels demonstrated considerable roughness. By visual inspection it would appear this roughness in parts could be as large as $200-400 \mu \mathrm{m}$. Considering the diameter of the channel was only $2000 \mu \mathrm{m}$, it is clear how this roughness could lead to substantial blockages when trying to remove non-melted powder.

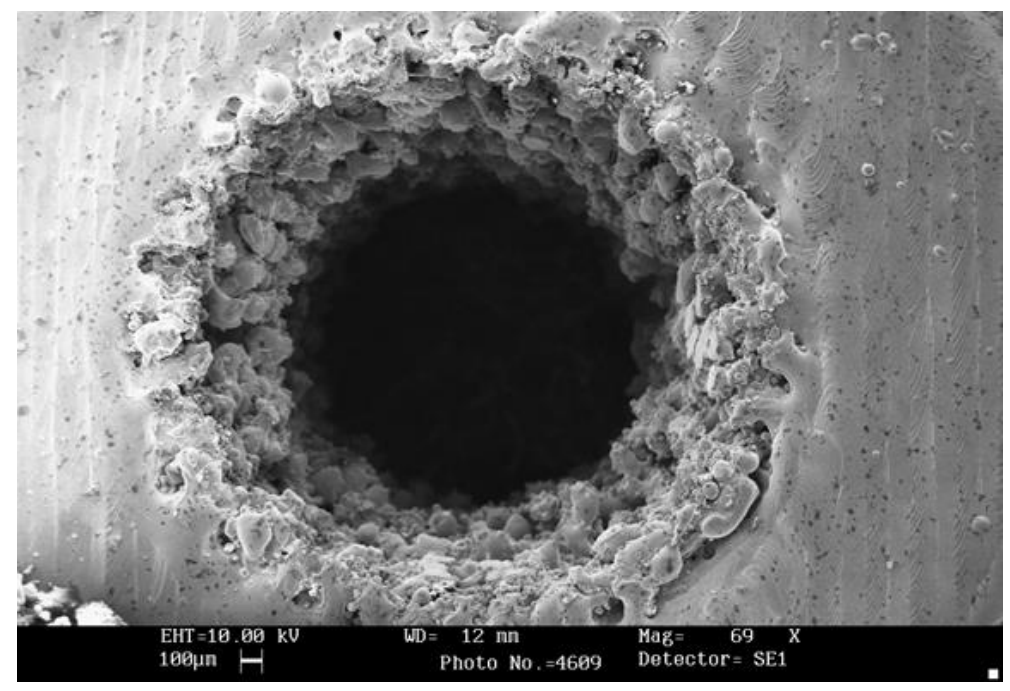

Figure 77: SEM image of section of part 3D built in the $\mathrm{z}$ plane.

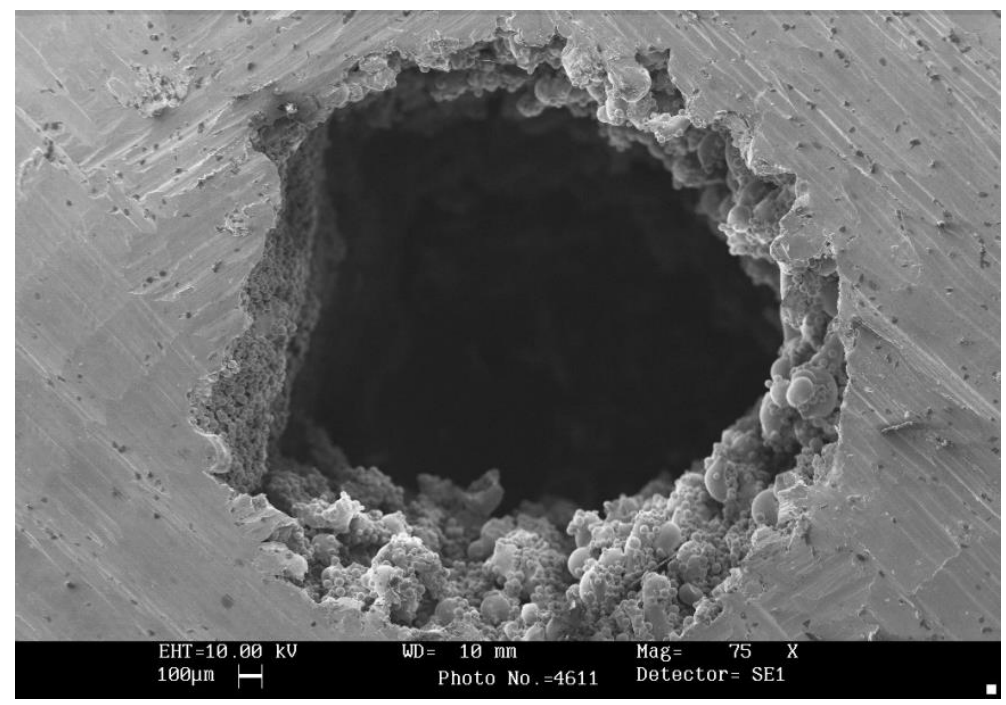

Figure 78: SEM image of section of part 3D built in the $x-y$ plane. 
In order to visualise the surface of the channel perpendicular to the microscope or face on, this section was segmented using a hack saw. The images showed us that the surfaces of the parts were covered in large agglomerations of melted powder, each of which had many smaller nodules of powder attached (Figure 79). Some of these larger agglomerations had melted together forming areas of considerable surface roughness. The surface roughness of the parts did appear to be reasonably uniform across the entire surface of the channel.

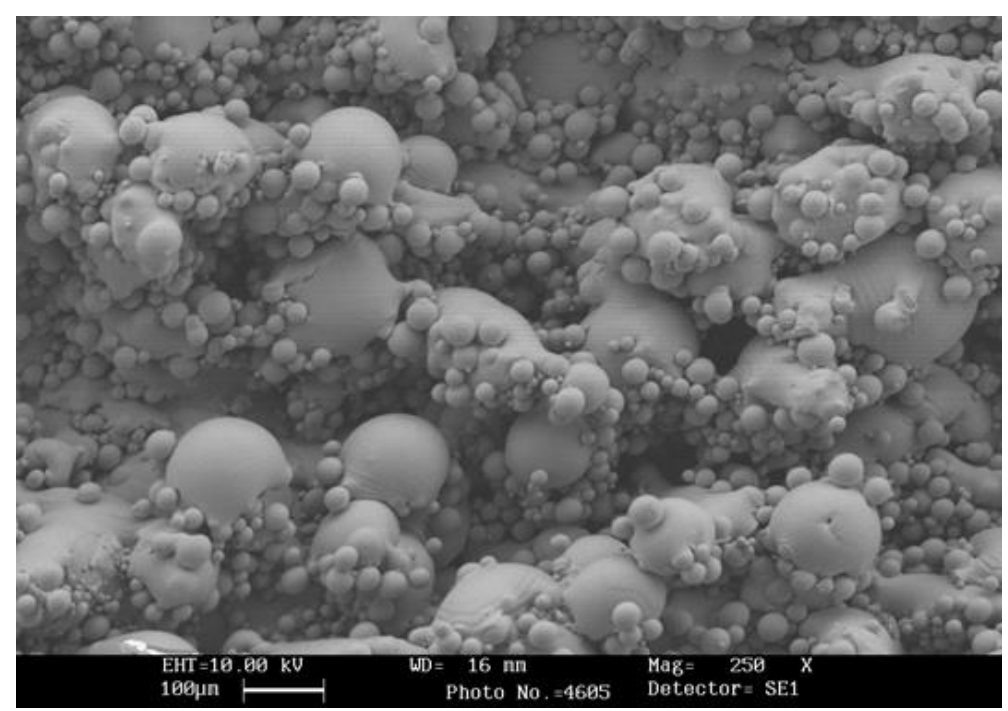

Figure 79: SEM image of section of part 3D built in the $\mathrm{z}$ plane.

In order to quantify this surface roughness, a non-contact scanning topography, Talysurf CLI 2000 Machine 3D surface profiler was used (Figure 80). The TalySurf CCI is an advanced 3dimensional optical metrology tool used for surface characterisation. The machine visualises individual points on the surface of the part, measuring the altitude of the surface at each point. It repeats this process over the entire surface of the part, before reconstructing the values into a 3-D model of the surface. Although the relatively small $1 \mathrm{~mm}^{2}$ area which was measured may not be a true representation of the entire build, it does give us an indication of the level of surface roughness the SLM process is generating. The majority of peaks and troughs on the surface were in the region of $0-200 \mu \mathrm{m}$, however there were clearly areas where this roughness may be in the region of $400-800 \mu \mathrm{m}$. It is clear that this level of roughness within a $2 \mathrm{~mm}$ channel would have a major impact on the removal of non-melted powder from within the part. This level of roughness is comparable with the values measured on the light microscopy and SEM image analysis 

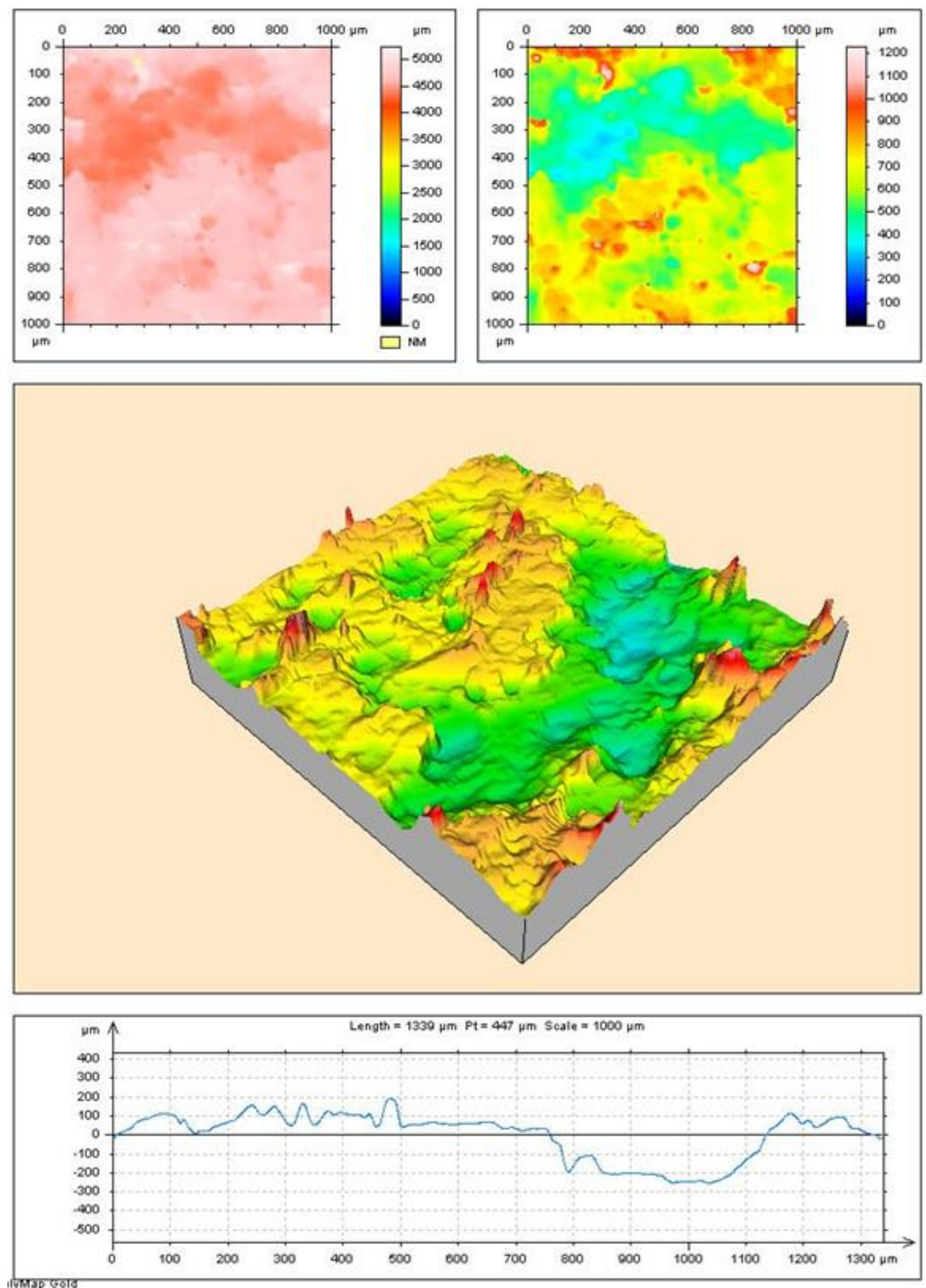

Figure 80: Surface representation of the section of part built in the $Z$ plane. Top: 2D colour map of surface topography, middle: 3D representation of the part surface, bottom: graphic representation of the analysed data. 
The SEM was also used to visualise the surface of the section of tube manufactured in the $x-y$ plane, i.e. the axis of the tube manufactured was parallel to the $x-y$ plane (Figure 78). Although the part demonstrated uniform surface roughness around 3 sides of the build (nominally the roof and side walls), similar to that seen in the part manufactured in the $\mathrm{Z}$ plane, the floor of the channel did not demonstrate the same level of roughness. Indeed, when the magnification of the SEM was increased to visualise this surface, individual powder particles could be seen bonded to the surface of the channel (Figure 75). During the manufacturing process, the section of build defining the floor of the channel would only come into contact with fresh powder particles when a new layer had been deposited over the top of the build by the powder wiper. This area of the build has presumably now had time to cool down from the melting process, meaning there would only be enough energy for a fine layer of these powder particles to adhere to the surface of the part. However, as the walls begin to take shape the resulting channel will fill with non-melted powder particles. When the laser scans the area of metal defining the channel wall and ceiling, it will be in direct contact with this fresh non-melted powder. The considerably temperature and energy within this region may well be leading to much larger quantities of powder binding to the surface of the build. This theory also explains why parts built in the $\mathrm{z}$ plane demonstrate uniform surface roughness. Undoubtedly, more detailed studies need to be conducted to determine the exact relationship between channel design and machine processing parameters, with the manufacturing accuracy and ultimately the ease of non-melted powder removal.

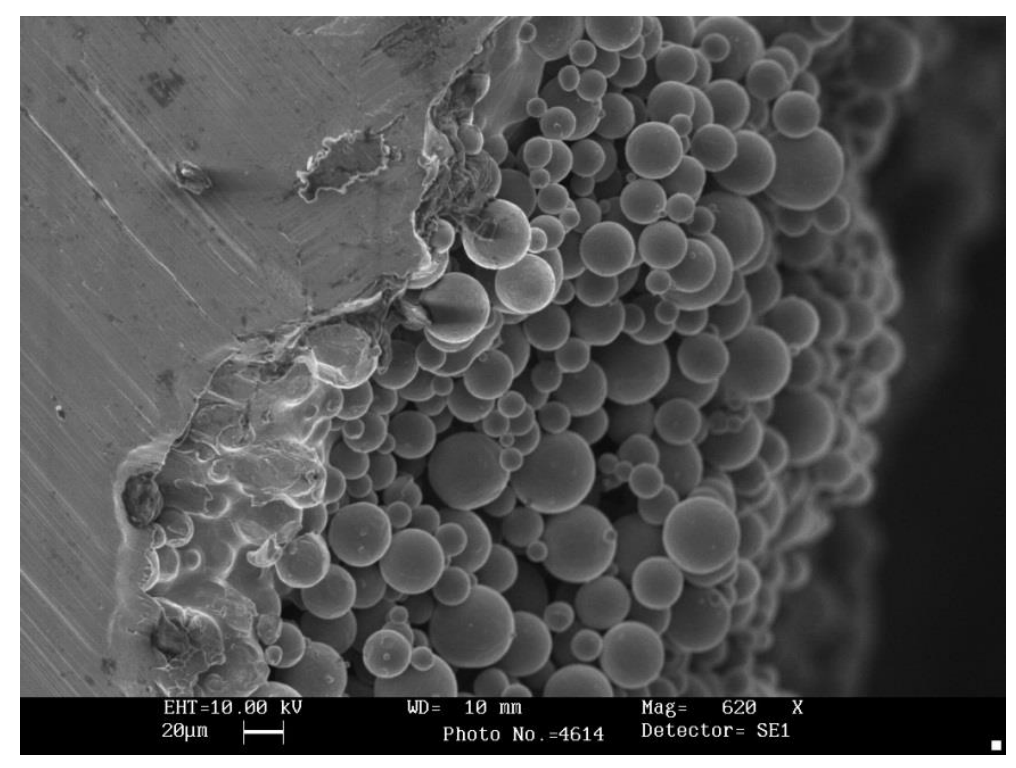

Figure 81: Expanded SEM image of a section of part 3D built in the X-Y plane. 
Measurements of the parts were taken in the $\mathrm{x}$ and $\mathrm{y}$ plane, comparing this data with the original CAD to determine dimensional accuracy. The variation in the measurements is consistently between 150 and $300 \mu \mathrm{m}$, which is an inherent feature of the surface roughness typically associated with parts manufactured via the SLM process. However, when expressed as a percentage variation it is clear that geometrical accuracy of SLM parts is at its greatest for larger dimensions. It could therefore be argued that SLM is currently not a suitable process for the fabrication of geometries with micron sized features, however with the constantly evolving commercial market producing machines capable of generating higher resolution parts, then these micron sized features will become more available with each passing year.

\begin{tabular}{|c|c|c|c|c|c|}
\hline Part & Dimension & $\begin{array}{c}\text { Actual } \\
\text { Measurement } \\
(\mathbf{m m})\end{array}$ & $\begin{array}{c}\text { CAD } \\
\text { measurement } \\
(\mathbf{m m})\end{array}$ & $\begin{array}{c}\text { Difference } \\
(+/-\mathbf{m m})\end{array}$ & $\begin{array}{c}\text { Variation } \\
(\%)\end{array}$ \\
\hline 3A & $\mathrm{x}$ & 5.30 & 5.00 & 0.30 & 6.00 \\
\hline 3B & $\mathrm{x}$ & 6.24 & 6.00 & 0.24 & 4.00 \\
\hline 3C & $\mathrm{x}$ & 5.23 & 5.00 & 0.23 & 4.60 \\
\hline 3D & $\mathrm{x}$ & 6.28 & 6.00 & 0.28 & 4.67 \\
\hline 3A & $\mathrm{y}$ & 17.24 & 17.00 & 0.24 & 1.41 \\
\hline 3B & $\mathrm{y}$ & 22.17 & 22.00 & 0.17 & 0.77 \\
\hline 3C & $\mathrm{y}$ & 49.30 & 49.00 & 0.30 & 0.61 \\
\hline 3D & $\mathrm{y}$ & 50.19 & 50.00 & 0.19 & 0.38 \\
\hline
\end{tabular}

Table 38: Optical Calliper Measurements of SLM Part 3.

\subsubsection{SLM Part 4}

\section{Design, build and post processing:}

Having demonstrated that it was possible to manufacture smaller lengths of rigid tubing via SLM, the next stage in our development would be to manufacture similar parts with much larger channel lengths and internal reaction volumes, which could potentially be used for functional flow chemistry. Two parts from Ti-6Al-4V alloy were produced using the Renishaw AM250, trialling a range of channel lengths and internal channel diameters. The dimensions of these parts are outlined in Table 39. 


\begin{tabular}{|c|c|c|c|c|}
\hline Part & Length $(\mathbf{m m})$ & $\begin{array}{c}\text { Internal Tube } \\
\text { Diameter }(\mathbf{m m})\end{array}$ & $\begin{array}{c}\text { External Tube } \\
\text { Diameter }(\mathbf{m m})\end{array}$ & $\begin{array}{c}\text { Internal } \\
\text { Reaction } \\
\text { Volume }(\mathbf{m L})\end{array}$ \\
\hline $4 \mathrm{~A}$ & 1280 & 1 & 5 & 1.01 \\
\hline $4 \mathrm{~B}$ & 1280 & 2 & 6 & 4.02 \\
\hline
\end{tabular}

Table 39: Dimensions of SLM Parts 4.

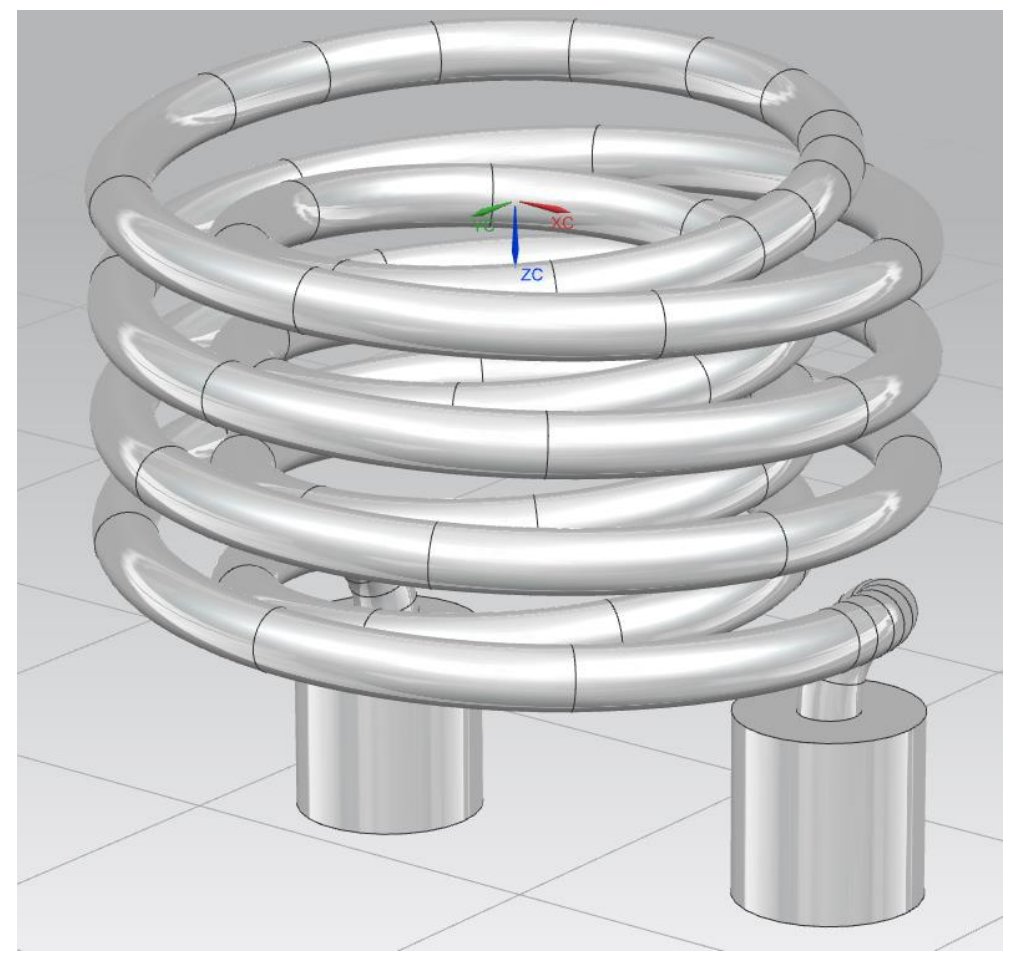

Figure 82: CAD image of SLM Part 4B.

\section{Application/Discussion}

The build was successful, with all non-melted powder easily removed from channels. Unfortunately, attempts to apply a thread to the build failed; the lack of bulk material surrounding the channels compromised the rigidity of the part, making it almost impossible to machine the channels. It may be possible to correct this issue by designing carefully constructed support structures around the channels. As well as this, the support structures automatically placed in by the SLM software to support the channels were not only aesthetically unpleasing but proved almost impossible to remove by conventional machining techniques. It was also noted that the lack of a defined shell to the part would inevitably cause issues with heating the part in a uniform manner on a conventional hot plate. 


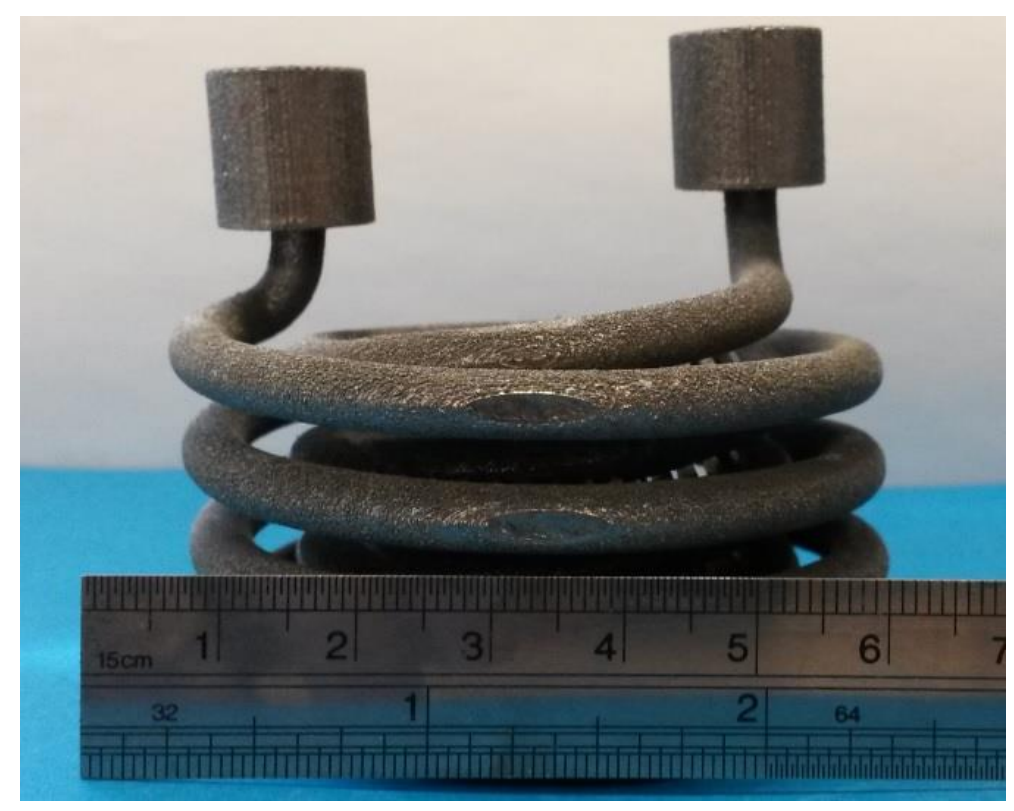

Figure 83: SLM Part 4.

Measurements of the parts were taken in the $\mathrm{x}$ and $\mathrm{z}$ plane, comparing this data with the original CAD to determine dimensional accuracy.

\begin{tabular}{|c|c|c|c|c|c|}
\hline Part & Dimension & $\begin{array}{c}\text { Actual } \\
\text { Measurement } \\
(\mathbf{m m})\end{array}$ & $\begin{array}{c}\text { CAD } \\
\text { measurement } \\
(\mathbf{m m})\end{array}$ & $\begin{array}{c}\text { Difference } \\
(+/-\mathbf{m m})\end{array}$ & $\begin{array}{c}\text { Variation } \\
(\boldsymbol{\%})\end{array}$ \\
\hline 4A & $\mathrm{x}$ & 4.22 & 4.00 & 0.22 & 5.50 \\
\hline 4B & $\mathrm{x}$ & 5.18 & 5.00 & 0.18 & 3.60 \\
\hline 4A & $\mathrm{y}$ & 64.12 & 64.00 & 0.12 & 0.18 \\
\hline 4B & $\mathrm{y}$ & 65.22 & 65.00 & 0.22 & 0.33 \\
\hline
\end{tabular}

Table 40: Optical Calliper Measurements of SLM Parts 4.

\subsubsection{SLM Parts 5}

\section{Design, build and post processing:}

The next stage in our SLM design development was to determine if it was possible to manufacture a part with an intricate channel network that would be difficult to manufacture using subtractive methods, without the inclusion of a large number of time consuming manufacturing steps or multiple smaller parts being connected together. Two parts were manufactured, the first containing a channel with baffles in order to encourage chaotic advection within the part, and the second a tube designed within another tube. This part 
would allow the channel to be jacketed by a heated/cooled fluid stream, leading to precise control of reaction thermodynamics. Both parts were manufactured from Ti-6Al-4V alloy using the Renishaw AM250. The dimensions of these parts are outlined in Table 41.

\begin{tabular}{|c|c|c|c|c|c|}
\hline Part & $\begin{array}{c}\text { Length } \\
(\mathbf{m m})\end{array}$ & $\begin{array}{c}\text { Internal Tube } \\
\text { Diameter } \\
(\mathbf{m m})\end{array}$ & $\begin{array}{c}\text { Internal Tube } \\
\text { Diameter 2 } \\
(\mathbf{m m})\end{array}$ & $\begin{array}{c}\text { External Tube } \\
\text { Diameter } \\
(\mathbf{m m})\end{array}$ & $\begin{array}{c}\text { Internal } \\
\text { Reaction } \\
\text { Volume (mL) }\end{array}$ \\
\hline $\begin{array}{c}\text { Inbuilt }- \\
\text { baffles }\end{array}$ & 100 & 5 & N/A & 7 & 1.75 \\
\hline $\begin{array}{c}\text { 5B }- \\
\text { Tube in a } \\
\text { tube }\end{array}$ & 75 & 2 & 4 & 6 & 0.24 \\
\hline
\end{tabular}

Table 41: Part dimensions for SLM parts 5.

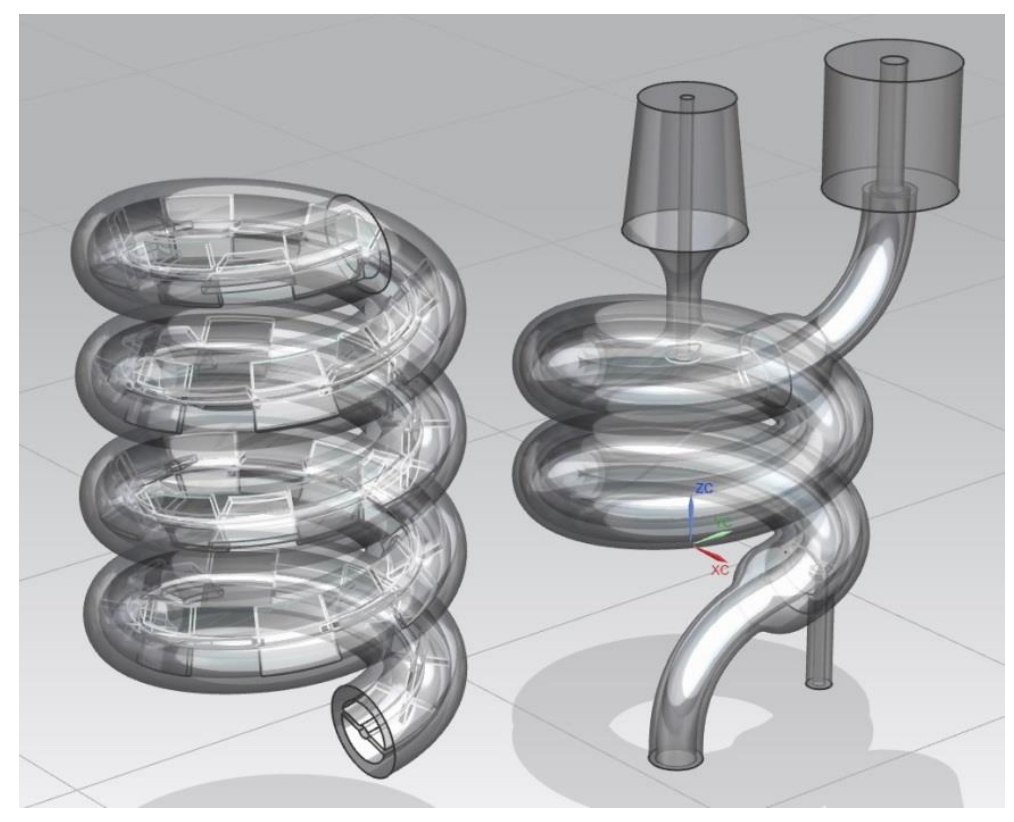

Figure 84: CAD images of SLM parts 5A and 5B. Left: Part with baffles throughout the channel, Right: Jacketed section of tubing.

\section{Application/Discussion:}

Unfortunately, during the manufacturing process the build failed due to an electrical error with the equipment. However, this did give us the opportunity to visualise the internal structures of the partially built parts. Whilst both parts were built geometrically accurately, it could again clearly be noted that there was substantial surface roughness. This roughness would make removal of powder from parts with this level of intricate internal features 
extremely difficult. Incorporation of these features would almost certainly need to be offset by an increase in channel dimensions towards parts with centimetre-scale geometries.

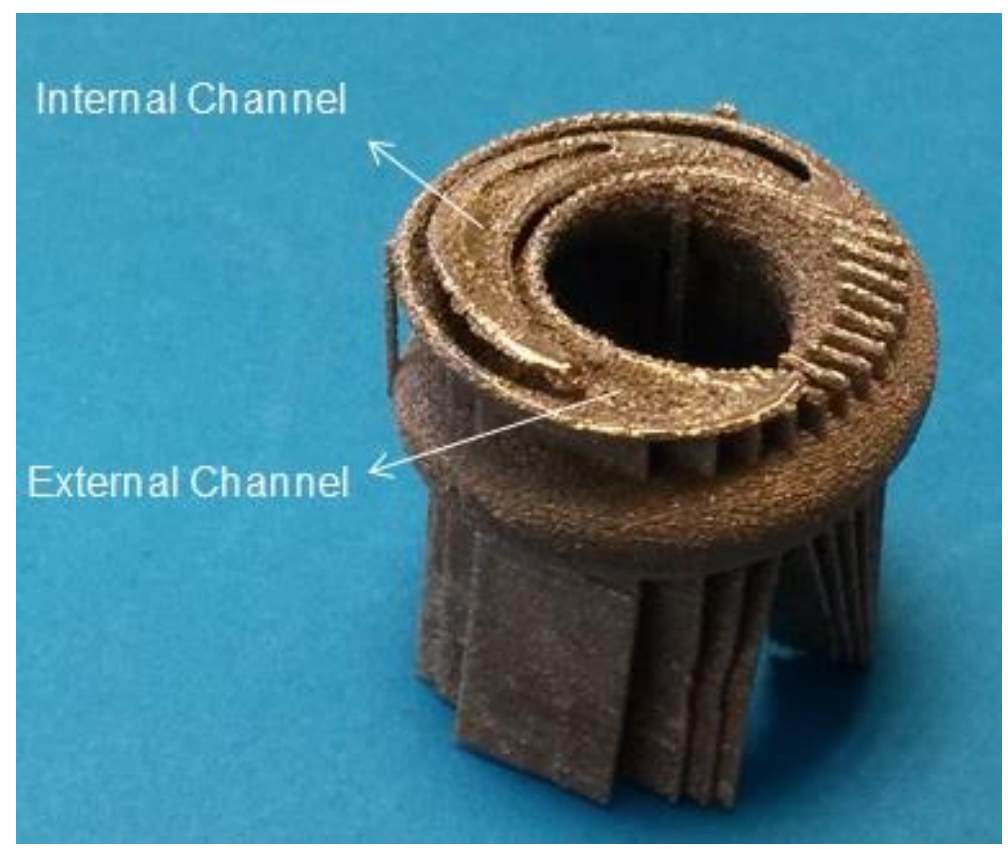

Figure 85: SLM part 5A.

Measurements of the part were taken in the $\mathrm{x}, \mathrm{y}$ and $\mathrm{z}$ plane, comparing this data with the original CAD to determine dimensional accuracy.

\begin{tabular}{|c|c|c|c|c|c|}
\hline Part & Dimension & $\begin{array}{c}\text { Actual } \\
\text { Measurement } \\
(\mathbf{m m})\end{array}$ & $\begin{array}{c}\text { CAD } \\
\text { measurement } \\
(\mathbf{m m})\end{array}$ & $\begin{array}{c}\text { Difference } \\
(+/-\mathbf{m m})\end{array}$ & $\begin{array}{c}\text { Variation } \\
(\%)\end{array}$ \\
\hline 5A & $\mathrm{x}$ & 7.27 & 7.00 & 0.27 & 3.86 \\
\hline 5B & $\mathrm{x}$ & 7.31 & 7.00 & 0.31 & 4.43 \\
\hline 5A & $\mathrm{y}$ & 27.33 & 27.00 & 0.33 & 1.22 \\
\hline 5B & $\mathrm{y}$ & 27.24 & 27.00 & 0.24 & 0.89 \\
\hline
\end{tabular}

Table 42: Optical calliper measurement of SLM Parts 5.

\subsubsection{SLM Part 6}

\section{Design, build and post processing:}

Previous work (SLM parts 1-5) with the Renishaw AM250 and Realizer SLM 100 had encountered significant issues with build resolution and surface finish. At this point in the research a new machine, the Realizer SLM 50, was available for use. The machine is slightly 
limited by the smaller build plate dimensions ( $80 \mathrm{~mm}$ diameter circular plate) allowing only the manufacture of smaller parts, however the lower power laser of the Realizer theoretically may be able to produce more precise builds with improved surface finish. This would be a consequence of less heat leakage into the powder bed, leading to less unwanted powder sintering. To determine the potential build resolution of the new SLM, a series of short channels of smaller channel diameters than had been previously attempted (SLM parts 1-5) were built. These helical channels would range in internal channel diameter from $1-2 \mathrm{~mm}$. The parts were manufactured from $316 \mathrm{~S}$ stainless steel with the geometries outlined in Table 43.

\begin{tabular}{|c|c|c|c|c|}
\hline Channel & $\begin{array}{c}\text { Length } \\
(\mathbf{m m})\end{array}$ & $\begin{array}{c}\text { Internal Tube } \\
\text { Diameter }(\mathbf{m m})\end{array}$ & $\begin{array}{c}\text { External Tube } \\
\text { Diameter }(\mathbf{m m})\end{array}$ & $\begin{array}{c}\text { Internal Reaction } \\
\text { Volume }(\mathbf{m L})\end{array}$ \\
\hline A & 68.5 & 2 & 3 & 0.22 \\
\hline B & 68.5 & 1.75 & 3 & 0.16 \\
\hline C & 68.5 & 1.5 & 3 & 0.12 \\
\hline D & 68.5 & 1.25 & 3 & 0.08 \\
\hline E & 68.5 & 1 & 3 & 0.05 \\
\hline
\end{tabular}

Table 43: Part dimensions for SLM part 6.

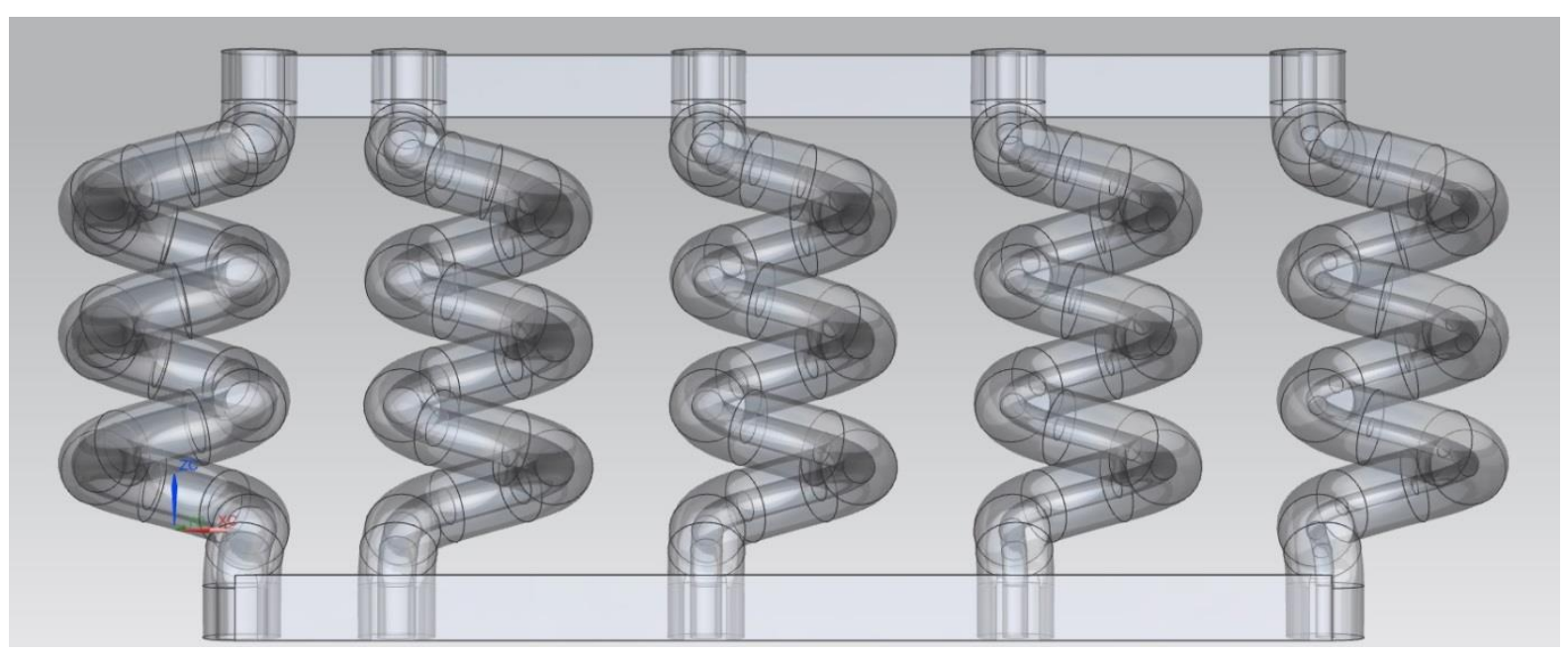

Figure 86: CAD images of SLM part 6.

\section{Application/Discussion}

The build was successful, with all non-melted powder easily removed from the channels. It was immediately noticeable that the part demonstrated significantly improved surface finish as well as geometrical accuracy. This was particularly noticeable on the intricate mesh of 
support structures surrounding the build, whose submillimetre sized mesh features had been built without visible signs of considerable surface roughness.

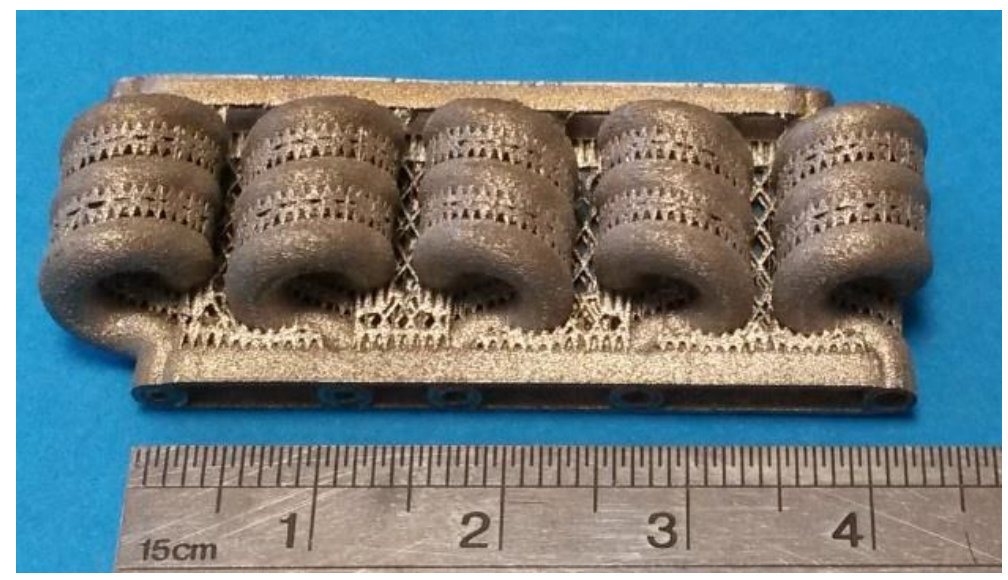

Figure 87: SLM Part 6.

Measurements of the part were taken in the $\mathrm{x}, \mathrm{y}$ and $\mathrm{z}$ plane, comparing this data with the original CAD to determine dimensional accuracy.

\begin{tabular}{|c|c|c|c|c|}
\hline Dimension & $\begin{array}{c}\text { Actual } \\
\text { Measurement }(\mathbf{m m})\end{array}$ & $\begin{array}{c}\text { CAD measurement } \\
(\mathbf{m m})\end{array}$ & $\begin{array}{c}\text { Difference } \\
(+/-\mathbf{m m})\end{array}$ & $\begin{array}{c}\text { Variation } \\
(\boldsymbol{\%})\end{array}$ \\
\hline $\mathrm{X}$ & 2.48 & 2.50 & 0.02 & 0.81 \\
\hline $\mathrm{Y}$ & 42.92 & 43 & 0.08 & 0.19 \\
\hline $\mathrm{Z}$ & 20.44 & 20.50 & 0.06 & 0.29 \\
\hline
\end{tabular}

Table 44: Optical Calliper Measurements of SLM Part 6.

\subsubsection{SLM Part 7}

\section{Design, build and post processing:}

Having demonstrated that it was possible to manufacture complex features using the Realizer SLM 50, a part with a complex internal channel network was manufactured which had sufficient internal reaction volume to be used for functional flow chemistry. Due to material availability this part was manufactured from Ti-6Al-4V alloy, with a complex offset triangular channel network. The triangular channel cross section was an equilateral triangle with sides of length $4.1 \mathrm{~mm}$, whereby the triangular cross section was offset by $180^{\circ}$ every 10 $\mathrm{mm}$. This is a geometry that had been successfully manufactured using the more reproducible SL process, and would demonstrate the accuracy and reproducibility of the SLM process. 


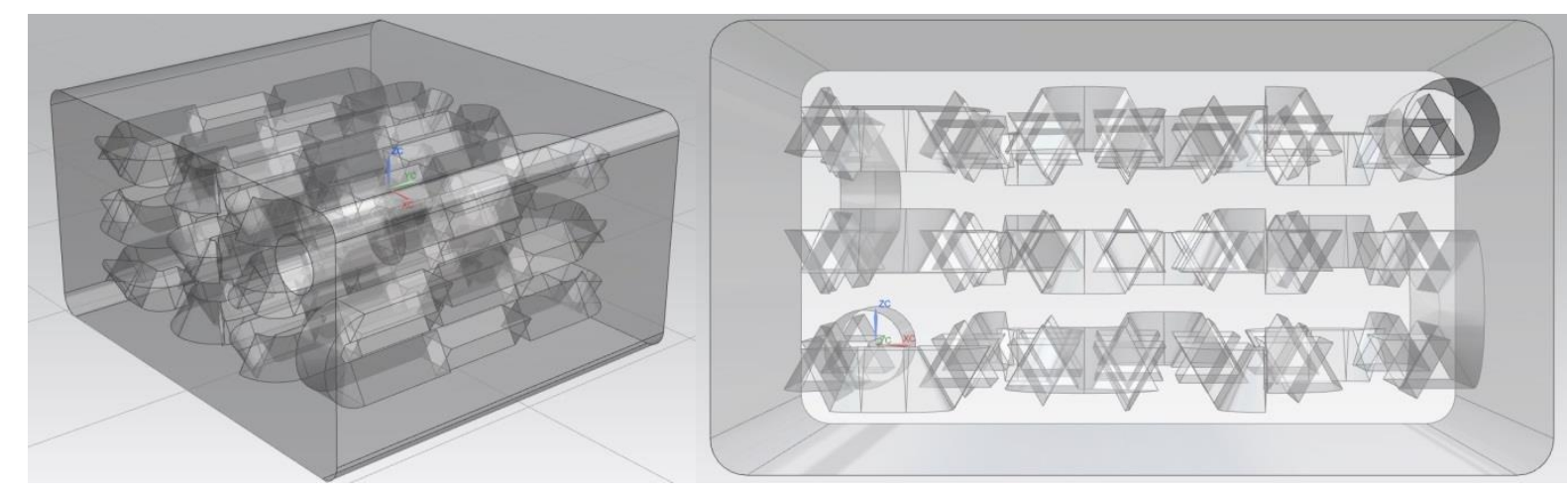

Figure 88: CAD images of SLM part 7.

The part was built with the dimensions outlined in Table 45.

\begin{tabular}{|c|c|}
\hline Part Length $(\mathrm{mm})$ & 41.75 \\
\hline Part Width $(\mathrm{mm})$ & 46.00 \\
\hline Part Height $(\mathrm{mm})$ & 24.80 \\
\hline Part Volume $\left(\mathrm{cm}^{3}\right)$ & 41.38 \\
\hline Channel Cross Sectional Area $\left(\mathrm{cm}^{2}\right)$ & 0.07 \\
\hline Channel Length $(\mathrm{mm})$ & 800 \\
\hline Internal Reaction Volume $\left(\mathrm{cm}^{3}\right)$ & 5.81 \\
\hline
\end{tabular}

Table 45: SLM Part 7 Build Dimensions.

\section{Application/Discussion}

The build was successful, with all non-melted powder easily removed from the channels. Although the surface of the part still had a slightly grainy texture, surface roughness was considerably reduced from all parts previously constructed on the Renishaw AM250. Unfortunately though, when attempting to pump solvent through the reactor, the part developed a hair line fracture along the surface, causing the part to leak at this point. The cause of this crack was investigated further. 


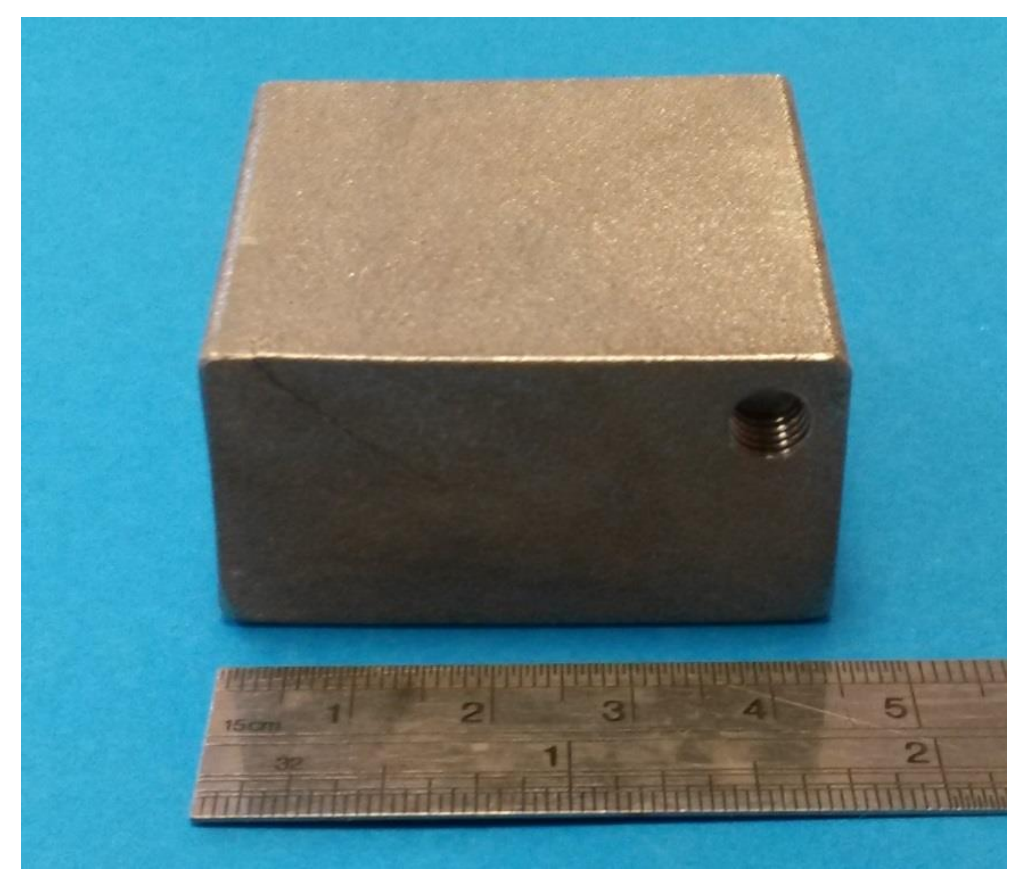

Figure 89: SLM Part 7

In order to visualise both the complex internal structures of the part, as well as the source of the fracture, the part was imaged via X-ray micro computed tomography $(\mu \mathrm{CT})$. This technique would also allow the quantification of the geometrical build resolution of the internal features of our parts for the first time. $\mu \mathrm{CT}$ uses X-ray imaging and computed tomography to produce $3 \mathrm{D}$ images of a sub-micron resolution. The $\mu \mathrm{CT}$ used was a XRADIA Versa XRM - 500 microscope, which is capable of visualising part geometries at a submicron level. The part was exposed to the X-Ray source for $15 \mathrm{~h}$ producing approximately 2500 images. Each image represents a cross sectional slice of the part of approximately 50 $\mu \mathrm{m}$ thickness. These images can then be imported into the open access Java based image processing software Image $\mathrm{J}$, allowing $3 \mathrm{D}$ reconstruction of the part. 


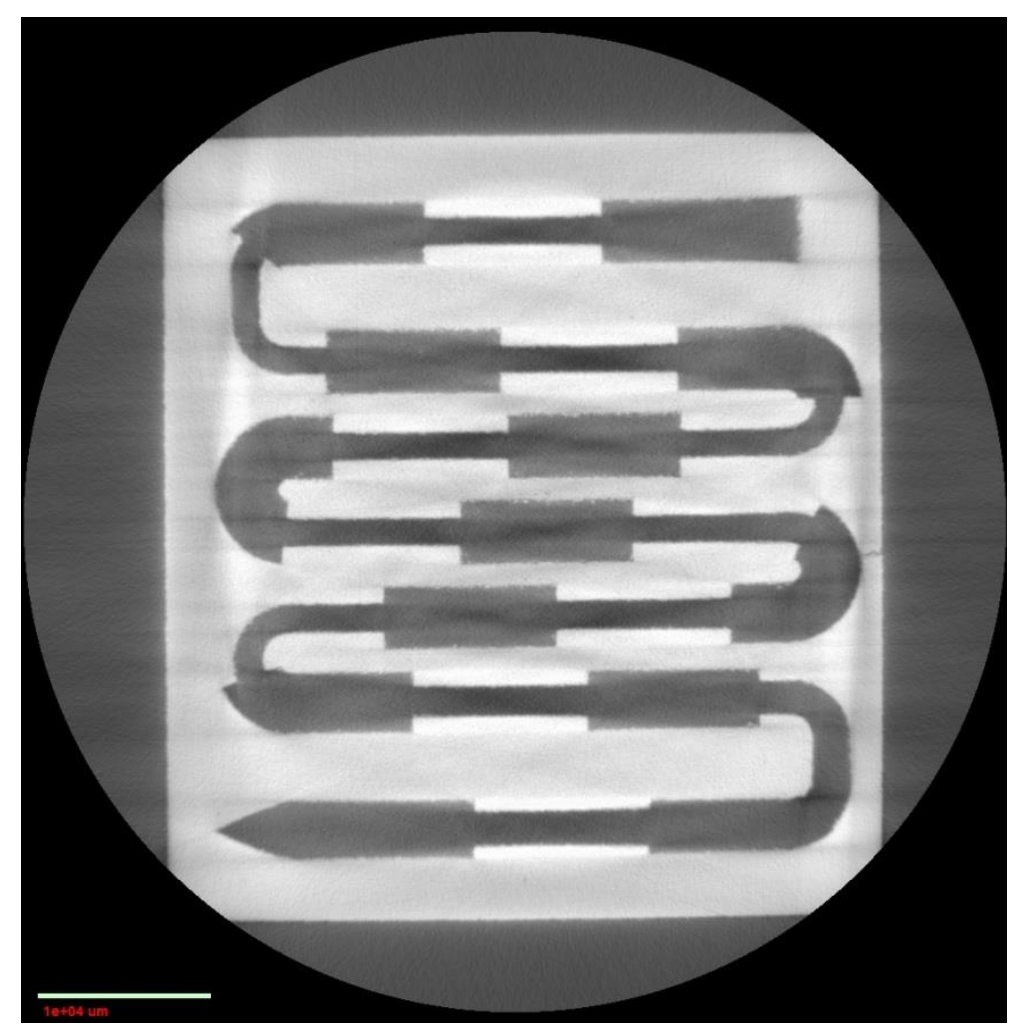

Figure 90: X-ray microCT scan of a single layer of SLM part 7 in the xy plane.

Visualisation of the part in the xy plane clearly shows the density of the solid body surrounding the channel. This once again demonstrates that SLM is capable of producing completely non-porous dense parts. The images in this plane also clearly demonstrate that the part has been completely cleared of non-melted powder, and that the cross section of the part is alternating every $10 \mathrm{~mm}$. By determining the size of each pixel within the image as being $57.47126 \mu \mathrm{m}$, it was possible to measure the size of each channel. Fifteen channel sections were selected from within the part with which we could gain a representation of the overall part. The channel measurements for these regions are as outlined in Table 46. 


\begin{tabular}{|c|c|c|}
\hline Channel Number & Number of Pixels & Channel Size $(\mathrm{mm})$ \\
\hline 1 & 73 & 4.20 \\
\hline 2 & 71 & 4.08 \\
\hline 3 & 71 & 4.08 \\
\hline 4 & 67 & 3.85 \\
\hline 5 & 69 & 3.97 \\
\hline 6 & 67 & 3.85 \\
\hline 7 & 68 & 3.91 \\
\hline 8 & 71 & 4.08 \\
\hline 9 & 69 & 3.97 \\
\hline 10 & 69 & 3.97 \\
\hline 11 & 69 & 3.97 \\
\hline 12 & 71 & 4.08 \\
\hline 13 & 70 & 4.02 \\
\hline 14 & 70 & 4.02 \\
\hline 15 & 72 & 4.14 \\
\hline & Average & 4.01 \\
\hline
\end{tabular}

Table 46: Channel Dimensions of SLM Part 7 as viewed in the xy Plane.

The images showed the channel to be between 68 and 73 pixels in diameter, although limitations in image resolution make differentiation of the outer pixels more difficult to quantify. This gave an average channel size of $4.01 \mathrm{~mm}$ with a standard deviation of $0.1 \mathrm{~mm}$. Due to the dimensions of the channel changing in each image of this series, it is difficult to select the exact point at which the channel diameter is maximal. These measurements give the channels a theoretical width of between 3.85 and $4.20 \mathrm{~mm}$, with an average value of 4.01 $\mathrm{mm}$. This puts the part at $2.2 \%$ smaller than the CAD drawing specifies. In this image it is also possible to visualise the hairline fracture in the part, which appears to originate from the corner of one of the outermost triangular architecture within the part. 


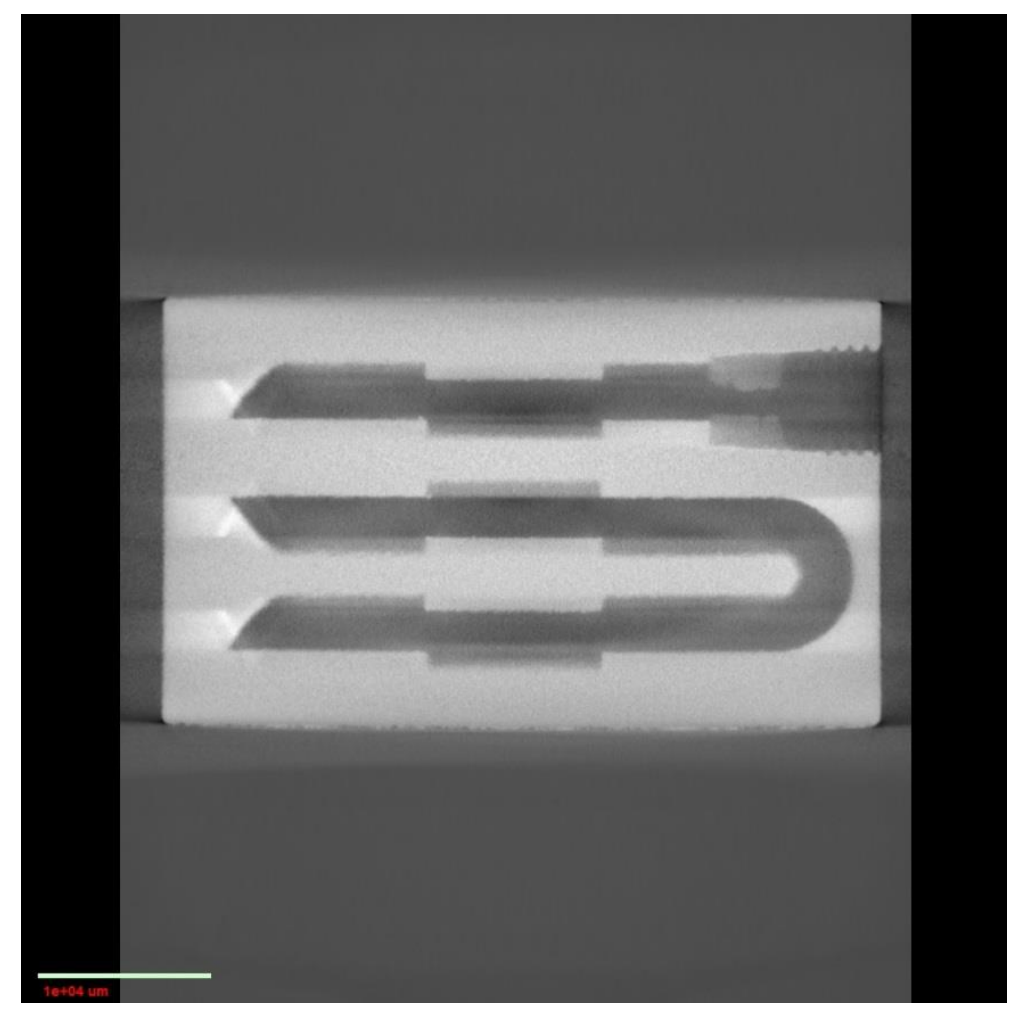

Figure 91: X-ray microCT scan of a single layer of SLM part 7 in the XZ plane.

Imaging in the xz plane also allowed us again determine the dimensions of the channel. Nine channel sections were again selected from within the part with which we could gain a representation of the overall part. The images follow a single cross section of channel for several $\mathrm{mm}$ in length, removing any of the difficulties associated with selecting the optimum image. These measurements will be for the height of the channel which should be $3.55 \mathrm{~mm}$ in value. The channel measurements for these regions are outlined in Table 47. 


\begin{tabular}{|c|c|c|}
\hline Channel Number & Number of Pixels & Channel Size (mm) \\
\hline 1 & 62 & 3.56 \\
\hline 2 & 59 & 3.39 \\
\hline 3 & 63 & 3.62 \\
\hline 4 & 62 & 3.56 \\
\hline 5 & 58 & 3.33 \\
\hline 6 & 63 & 3.62 \\
\hline 7 & 60 & 3.45 \\
\hline 8 & 62 & 3.56 \\
\hline 9 & 62 & 3.56 \\
\hline & Average & 3.52 \\
\hline
\end{tabular}

Table 47: Channel Dimensions of SLM Part 7 as viewed in the xz Plane.

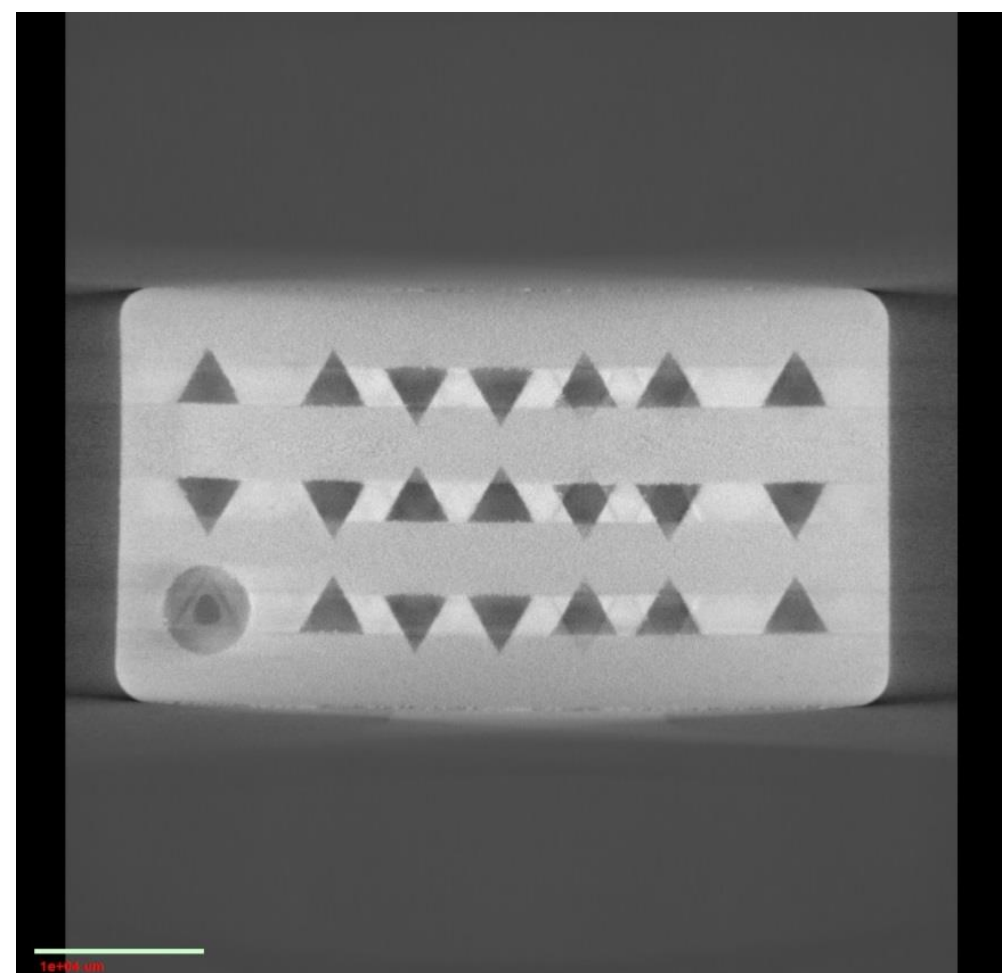

Figure 92: X-ray micro CT scan of a single layer of SLM part 7 in the yz plane.

The images showed the channel to be between 58 and 62 pixels at its maximum width, giving the channels a theoretical width of between $3.33-3.62 \mathrm{~mm}$, with an average value of $3.52 \mathrm{~mm}$ (standard deviation $0.1 \mathrm{~mm}$ ). This puts the part at $0.9 \%$ smaller than the CAD drawing specifies. 
The images taken in the yz plane make determination of channel dimensions even more simplistic. The images follow a single cross section of channel for $10 \mathrm{~mm}$ in length, removing any of the difficulties associated with selecting the correct image. Theoretically, visualisation in this plane should provide the most accurate representation of the channel dimensions. Again nine images were selected across the area of the part with the dimensions outlined in Table 48.

\begin{tabular}{|c|c|c|}
\hline Channel Number & Number of Pixels & Channel Size (mm) \\
\hline 1 & 59 & 3.39 \\
\hline 2 & 62 & 3.56 \\
\hline 3 & 59 & 3.39 \\
\hline 4 & 61 & 3.51 \\
\hline 5 & 65 & 3.73 \\
\hline 6 & 62 & 3.56 \\
\hline 7 & 62 & 3.56 \\
\hline 8 & 61 & 3.51 \\
\hline 9 & 62 & 3.56 \\
\hline & Average & $\mathbf{3 . 5 3}$ \\
\hline
\end{tabular}

Table 48: Channel Dimensions of SLM Part 7 as viewed in the yz Plane.

The images showed the channel to be between 59 and 65 pixels at its maximum width, giving the channels a theoretical width of 3.39-3.73 mm, with an average value of $3.53 \mathrm{~mm}$ (standard deviation $0.1 \mathrm{~mm}$ ). This puts the part at $0.6 \%$ smaller than the CAD drawing specifies. It is noticeable that the error of the features built in the xy plane (2.2\%), was greater than those built in the $\mathrm{xz}(0.9 \%)$ and the $\mathrm{yz}(0.6 \%)$. Presumably this is a consequence of these feature accuracies being dependant on the machine optics, whereas the $\mathrm{xz}$ and $\mathrm{yz}$ features would be dependent on the mechanics of the build platform.

It is also possible to use the Image $\mathbf{J}$ software to form a 3D reconstruction of the part using the images from the micro CT. This reconstruction does rely on the exact definition of what is signal and what is background noise, which can lead to the introduction of errors into reconstructed images. This reconstruction relies on defining the threshold of individual pixel colours which are to be included within the count. This error will increase as the time and number of X-ray scans is reduced. This reconstruction can be exported as an .STL file format and opened in any CAD software for visualisation (Figure 87). 


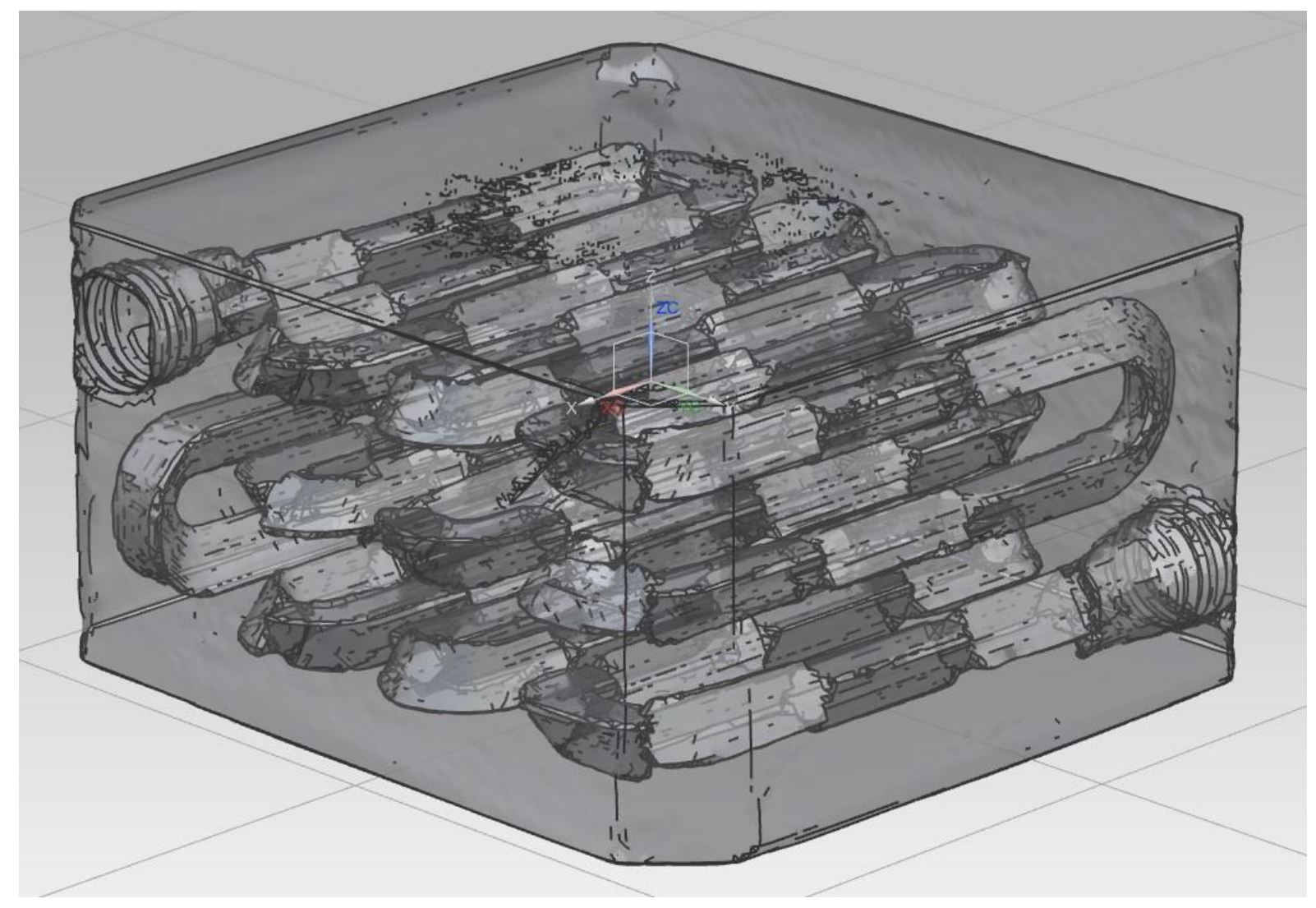

Figure 93: Reconstructed CAD image of X-ray micro CT scan of SLM part 7.

The reconstruction allows us to clearly visualise and trace the hairline fracture within the part; it originates from the corner of one of the outer most triangular cross section of the channel. The fracture may have been caused by a simple defect in the build process or it could have been consequence of the design of channel network. The sharp corners of the triangular cross section may have created a natural focus for any pressure exerted on the part during high pressure flow processes, making this type of fracture more likely. Also this section of the build has one of the thinnest sections of metal surrounding the channel, increasing the possibility of this type of breakage. Future designs should perhaps looks to eradicate this type of sharp design by rounding the edges of any channel cross sections which contained pointed features. 


\begin{tabular}{|c|c|c|c|c|}
\hline Dimension & $\begin{array}{c}\text { Actual Measurement } \\
(\mathbf{m m})\end{array}$ & $\begin{array}{c}\text { CAD measurement } \\
(\mathbf{m m})\end{array}$ & $\begin{array}{c}\text { Difference (+/- } \\
\mathbf{m m})\end{array}$ & $\begin{array}{c}\text { Variation } \\
(\boldsymbol{\%})\end{array}$ \\
\hline $\mathrm{X}$ & 41.74 & 41.75 & 0.01 & 0.02 \\
\hline $\mathrm{Y}$ & 45.94 & 46.00 & 0.06 & 0.13 \\
\hline $\mathrm{Z}$ & 24.85 & 24.80 & 0.05 & 0.20 \\
\hline
\end{tabular}

Table 49: Optical Calliper Measurements of SLM Part 6.

A number of other SLM parts have been built during the course of this research, however they are discussed in more detail during Chapter 7.

In general, dimensional accuracy of parts built via the SLM process using a Renishaw AM250 system was poor. Of the four parts manufactured, dimensions were on average within $330 \mu \mathrm{m}$ of the intended dimensions. However, the variation in dimensions always saw the part as larger than designed regardless of the size of the part, again suggesting the dimensional discrepancy was a factor of surface roughness of the parts.

The dimensional accuracy of parts built via the SLM process using a Realizer SLM 100 system was much better. The part that was built had dimensions that were on average within $73 \mu \mathrm{m}$ of the intended dimensions. Unfortunately, a number of reliability issues with this machine during the research led to use of the Renishaw machine for the majority of parts.

Finally, parts manufactured using the Realizer SLM 50 had excellent geometrical accuracy. The parts which were built had dimensions which were on average within $47 \mu \mathrm{m}$ of the intended dimensions. Future work would undoubtedly look to utilise this more accurate and reproducible machine.

\subsection{Conclusion}

Despite the obvious limitations of using SLM to manufacture complex channel networks, the process is potentially the most promising long term additive method for this application. The wide range of thermally and chemically stable materials which SLM is capable of processing means that fluidic devices manufactured via SLM would be applicable to a wide range of flow applications. With the development of less expensive bench top machines such as the Realizer SLM 50 used during this research, SLM is becoming more accessible to research and development teams around the world. If this development can lead to more robust build processes, which are capable of manufacturing parts with a build quality that is comparable to 
existing fluidic manufacturing processes, then SLM will undoubtedly be adopted as a suitable manufacturing process for this application.

\subsection{Future Work}

During the course of this research, this research has demonstrated that a key advantage of manufacturing using SLM is the stability of the parts produced to a range of extreme thermal and chemical conditions. Future research will need to be conducted in to how to optimise the design and manufacturing process to overcome the fundamental engineering issues associated with this process, such as powder and support structure removal. Future work will focus on developing metal flow devices with increased functionality, including inbuilt analysis points, temperature control via embedded thermocouples, and thermally controlled reactor regions. 


\section{Reaction Monitoring and Optimisation}

\subsection{Introduction}

Experimental design or design of experiment (DoE) within a chemical system is the process of adjusting the controllable variables until the optimal response, within the limitations of the system, is found. ${ }^{159}$ There are two basic types of experimental design: sequential and simultaneous. For multifactor responses, such as those encountered in flow chemistry, simultaneous or factorial designs are preferable because the interactions between each of the variables can be detected and accounted for. Typically these responses are often a quantifiable goal such as reaction cost or yield, and the parameters are controllable factors such as reaction temperature or pressure. ${ }^{160}$ The classical experimental approach to this is to change one variable at a time, whilst the other variables are held constant. This approach will work if each of the variables are independent of one another and give a linear response. However, there are some notable disadvantages to using this approach, most notably is the systems inability to discriminate between combination effects, and thus leading to an inability to highlight the true optimal conditions. Using a design of experiment approach is a highly systematic method for exploring any given reaction space; this reaction space is defined by the number of variables (e.g. pressure or $\mathrm{pH}$ ), where the optimal reaction parameters can be found by varying each of the factors simultaneously. For a full factorial design the number of experiments required is given by $2 \mathrm{x}$, where $\mathrm{x}$ is the number of factors and 2 is the number of levels. By systematically analysing these variables within the optimisation space, the optimum data point can be found. However, DOE is not a global optimisation tool as it can only be used to investigate a defined reaction space, which can be limited by the amount of experiments and therefore the time required to analyse this space. Where this type of approach is particularly useful is when there is already a significant amount of information available regarding the specific system being analysed, allowing a number or variables to be removed from the analysis. There are a number of examples of this type of optimisation being used in the literature for a range of applications. DoE has been utilised by Lonza Ltd as part of a standard methodology for optimising industrial reaction scale up, ${ }^{161}$ as well as being used to optimise the de-protection of the amine protecting $p$-methoxyphenyl group in an automated microreactor platform. ${ }^{162}$ 
There are now a number of more advanced optimisation methodologies are routinely used in flow chemistry and microfluidic applications, such as central composite ${ }^{163}$ and full factorial design. ${ }^{164}$ Of the several optimisation techniques available, potentially the most useful for flow applications are designs based on a class of figures known as simplexes.

A simplex is a geometrical figure which has a number of vertices (corners) equal to one greater than the number of dimensions in the factor space. ${ }^{165}$ For chemistry applications, each "dimension" represents a discrete variable within the experiment, e.g., temperature, pressure, acidity, etc. If $\mathrm{k}$ is the number of dimensions, then the simplex is defined as having $\mathrm{k}+1$ points in that space. ${ }^{166}$ Therefore, when two variables are being analysed, the simplex forms a three sided 2-D triangular figure. When three variables are being analysed the simplex represents a 3-D four sided tetrahedron. With more than three variables it becomes impossible to visualise the 4-D simplex created, however the methodology is still applicable. Each vertex of the simplex generated corresponds to a set of experimental conditions. ${ }^{167}$

The first step in the simplex method is to determine the response which will be generated, as well as the variables affecting that response. These variables must have clearly defined limits which the simplex is not allowed to go beyond. The user must also define the size of the original simplex. Larger simplexes are likely to overshoot the optimal point within the data set, whereas smaller simplexes are likely to be too small to differentiate between each data point response. Optimal simplex sizes should be approximately two to three times the error of each measurement. ${ }^{165}$ To explain this method, imagine a hypothetical scenario whereby the response is reaction yield, and the two variables are defined as temperature $\left(0-100^{\circ} \mathrm{C}\right)$ and $\mathrm{pH}(0-12)$. These reaction conditions will be run, producing a single response as yield. A plot of temperature versus $\mathrm{pH}$ will define a certain area, within which there will be a point of maximal response. Assume that three random points are selected on this plot (Figure 94), point $1(\mathrm{~T}=40, \mathrm{pH}=7)$, point $2(\mathrm{~T}=50, \mathrm{pH}=8)$ and point $3(\mathrm{~T}=50, \mathrm{pH}=6)$. The experiment for each of these data points is run, with the yields being determined as being $1=$ $40 \%, 2=50 \%, 3=60 \%$. The simplex method will then determine the new data point by selecting the lowest response, in this case point 1 , and creating a mirror image of this point through the line that intersects the remaining two points. In this instance this generates a new point $4(\mathrm{~T}=60, \mathrm{pH}=7)$. This new point defines a brand new simplex. The new set of experimental conditions is run, and the response of point 4 is measured as being $70 \%$. Had this response been lower than the other two remaining points the simplex would have mirrored itself and the new point would have been the same as point 1 . In this instance the 
simplex either finishes or a new simplex is randomly generated within the confines of the plot. In this instance however, the lowest data point response in the simplex defined by point 2, 3 and 4 is point 2. Point 2 is discarded and its mirror image point $5(\mathrm{~T}=60, \mathrm{pH}=5)$ is generated. By rejecting the least desirable response in each triangle and moving the simplex away from that point, the data set moves towards the point of optimum response. The simplex is moved or flipped around the plot until one of the following criteria is reached. If the simplex moves outside the defined limits of the plot, the new experiment should be performed with the same settings as the worst point in the previous simplex, and the second worst point would be mirrored in the following transformation. Finally, if the simplex reaches a pre-defined stopping criteria such as an optimal response or maximum number of data points, or if the simplex circles around a single data point that defines the optimal response of the data set. ${ }^{165}$ Upon satisfying a single one of these criteria the simplex will be stopped.

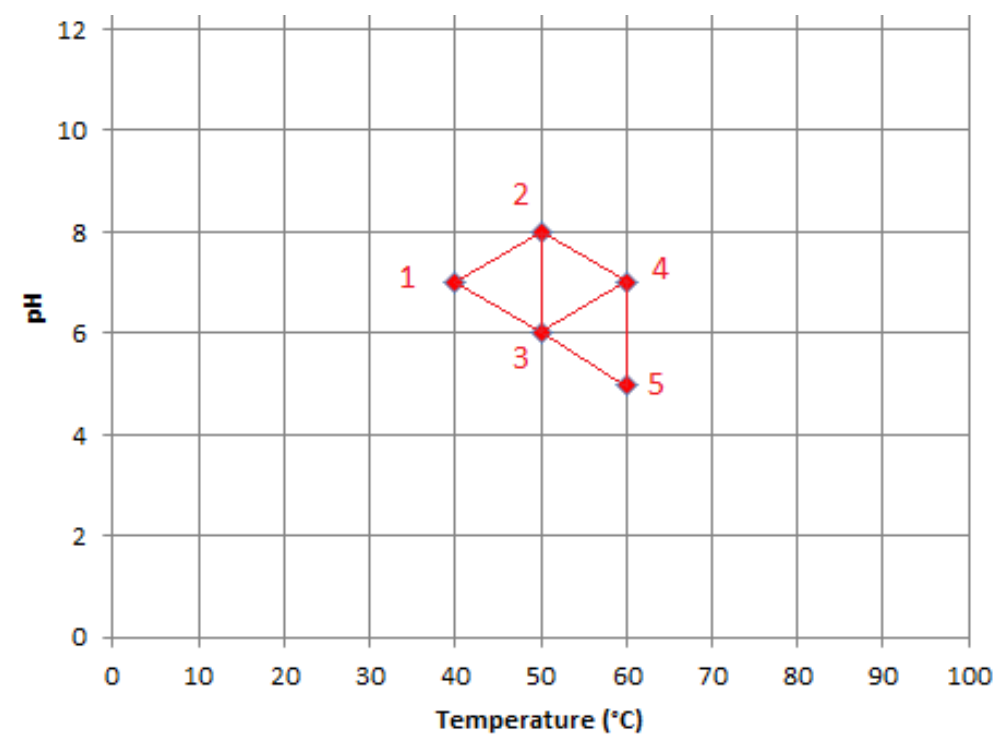

Figure 94: Progress of example simplex

Again there are a number of literature examples of this type of simplex optimisation for flow chemistry applications. The algorithm has been used as part of an optimisation of a Heck coupling in a continuous flow system, with the system variables being residence time and alkene molar equivalents. The group used online HPLC to provide the responsive output in the simplex methodology. ${ }^{168}$ Some of the most pioneering research in this area has been performed by the Poliakoff group at the University of Nottingham, who have used global optimisation algorithms for a wide range of diverse applications. Two dimensional correlation GC was used for the analysis and optimisation of a continuous flow catalytic Friedel-Crafts alkylation in supercritical CO2. ${ }^{169}$ Automated optimisation was demonstrated 
by the same group, using the Super Modified Simplex Algorithm (SMSIM) to locate an optimum set of reaction conditions for the methylation of alcohols with dimethyl carbonate. ${ }^{170}$ Each set of new reaction conditions were determined from the online analysis, and an optimising feedback procedure set the flow system up and ran the next experiment starting from an initial tetrahedron of conditions. Online spectroscopy is another key technology for online analysis and optimisation, primarily due to the speed of measurements and the structural information afforded through the analysis. The same research team used online ATR-FTIR with the SNOBFIT algorithm to optimise the methylation of 1-pentanol. ${ }^{171}$

AM presents a unique opportunity to develop bespoke flow devices which are capable of incorporating embedded sensors and detectors for inline analysis of reaction medium. The next stage of research development looked at the possibility of using SLM and SL processes to manufacture this type of analytical flow device, as well as using the design freedom of AM processes to produce parts which could be incorporated into existing flow and analytical equipment. These devices would allow the production of a wide range of quantitative analytical data. This data could be used as part of an inline experimental design software package, enabling simultaneous reaction optimisation to be performed. 


\subsection{Results and Discussion}

\subsubsection{SL Part 9}

\section{Design, build and post processing:}

The next stage of SL development would be the integration of analytical capability into devices. A simplistic method of incorporating spectroscopic capability would be by embedding a quartz window into the flow path of the cell. UV quartz is selected over glass for this application as it is UV transparent down to wavelengths of $180 \mathrm{~nm} .{ }^{172}$ This will allow analysis of the reaction medium across the entire UV-Vis-IR range. These windows can be purchased as part of a window cell which contains the quartz window itself, sealed in a compressible threaded container (M8 x 0.5 thread). This thread will allow easy integration and sealing of the window into devices. A part was designed and manufactured with a simplistic channel running throughout. The device had multiple points situated along the channel path, where these quartz windows could be incorporated. Into these analysis points it was possible to insert and seal the quartz windows, allowing light to pass through the reaction medium without disturbing the flow. The part was built with the dimensions outlined in Table 50.

\begin{tabular}{|c|c|}
\hline Part Length $(\mathrm{mm})$ & 100.00 \\
\hline Part Width $(\mathrm{mm})$ & 86.00 \\
\hline Part Height $(\mathrm{mm})$ & 23.30 \\
\hline Part Volume $\left(\mathrm{cm}^{3}\right)$ & 147.50 \\
\hline Channel Diameter $(\mathrm{mm})$ & 2.00 \\
\hline Channel Length $(\mathrm{mm})$ & 2400 \\
\hline Internal Reaction Volume $\left(\mathrm{cm}^{3}\right)$ & 7.20 \\
\hline
\end{tabular}

Table 50: SL Part 9 Build Dimensions.

The quartz windows were inserted by milling a $1 \mathrm{~mm}$ channel through the centre of the analysis ports, followed by two channels $(8 \mathrm{~mm}$ depth:7 $\mathrm{mm}$ diameter) from either side of the port. Future iterations would have these channels designed into the part. These two channels were tapped using an M8 x 0.5 thread, allowing a single quartz window to be inserted onto either side of the flow path. Initially a single window was placed into the device. 


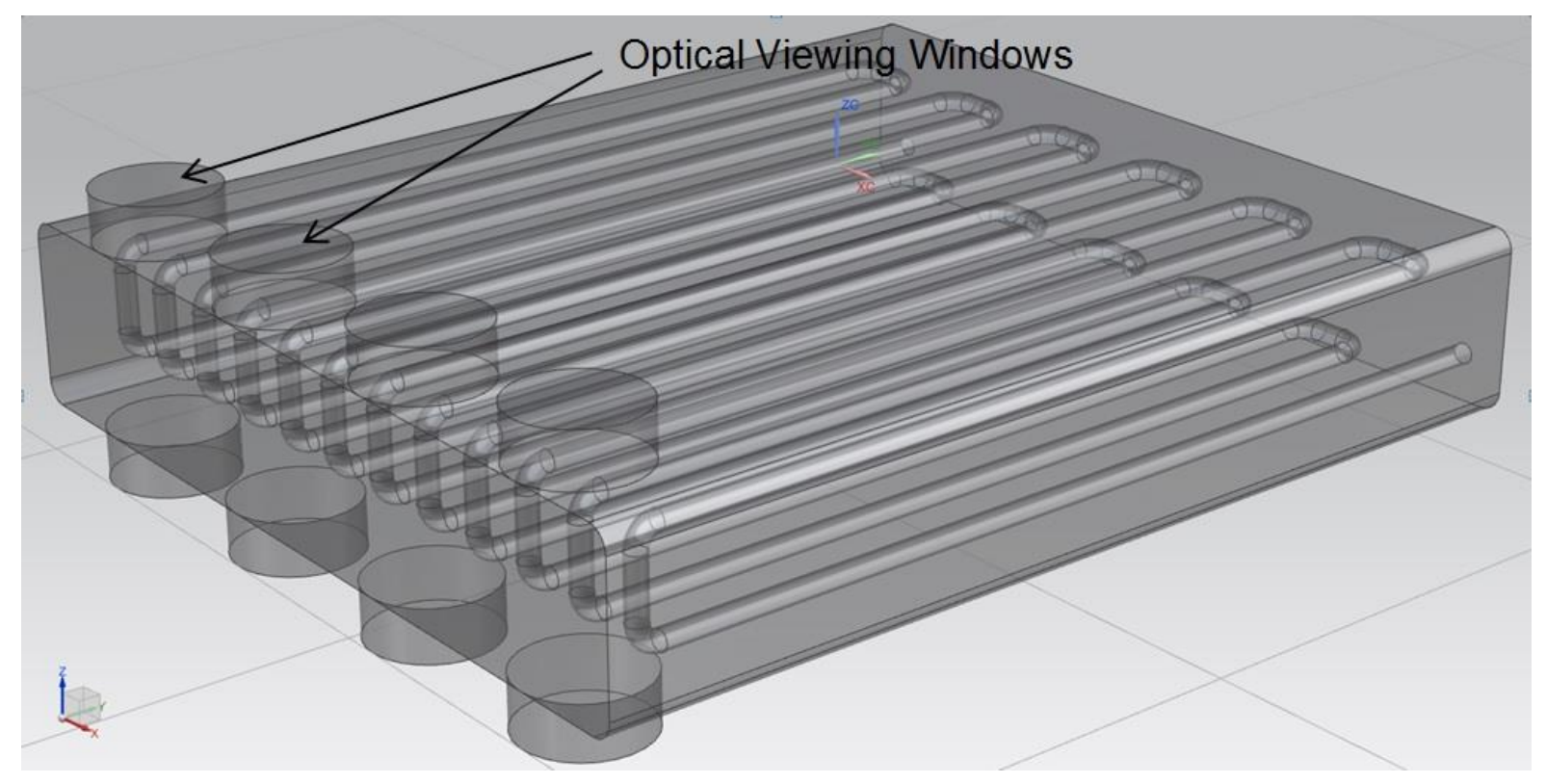

Figure 95: CAD model of SL part 9.

\section{Application/Discussion:}

A range of organic solvents were pumped through the part at flow rates ranging from $0.1-5$ $\mathrm{mL} / \mathrm{min}$, confirming that the part would not leak under elevated pressures. This part was never used for its spectroscopic capabilities (see SL Part 10), however it was used as a functioning flow reactor. In order to gain quantitative reaction data, it was proposed that using a high performance liquid chromatography (HPLC) type system as a method of monitoring the reaction medium online would be an ideal analysis method. Not only would this allow the separation and characterisation of any reaction products produced, but it would provide UV-Vis spectroscopy data of the reaction products, courtesy of the diode array detector (DAD) connected to the HPLC. A standard Agilent 1100 LC is supplied with a binary pumping module, an autosampler, a heated column compartment and a DAD compartment. It was proposed that it would be possible to use the pumping module, as well as the heated column compartment, to pump and control the temperature of the flow reactions. The column compartment has two separate temperature controlled compartments, which could be used to heat both a column and a milli-scale reactor. By acquiring a secondary pumping module, it would be possible to independently pump both the reaction medium through the system, as well as the LC mobile phase through the column. Almost all modern research laboratories have this type of LC system, which with a small amount of reformatting can be used as a continuous flow system with analytical capabilities. As well as the substantial cost saving over purchasing specialist flow apparatus, most chemists are 
familiar and comfortable using LC software, reducing the need for specialist training with the new equipment.

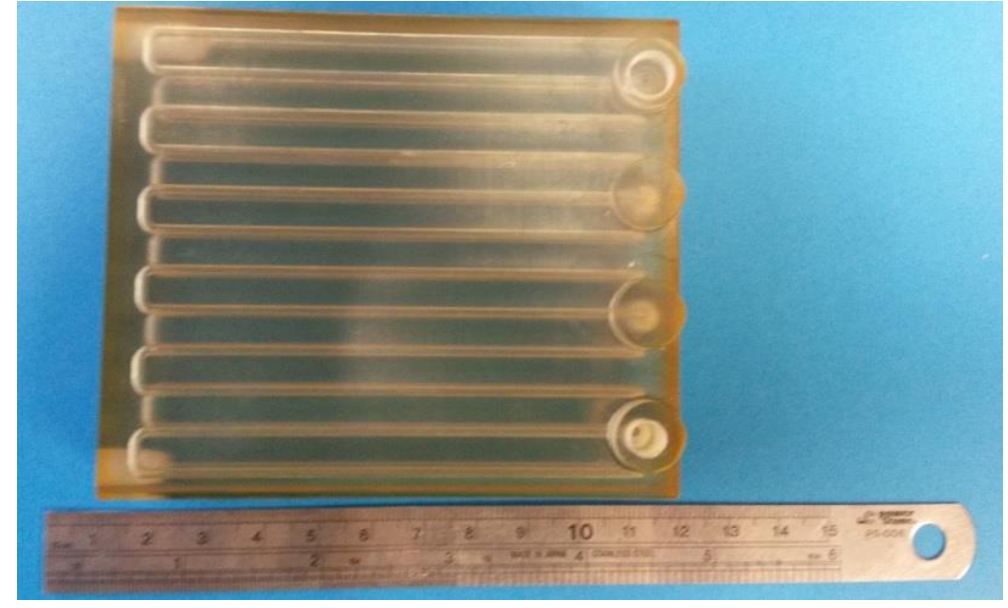

Figure 96: SL Part 9

After reconfiguring the HPLC, the equipment was setup for the specific needs of the experiment. The reaction medium would be pumped through the AM flow device by the first LC binary pumping module. This device could be placed externally to the system, or contained within the first of the two heated column compartments (part dimensions permitting). The outflow from the device would be passed directly into an external 6 port valve, which would direct the flow into an external sample collection vial. The 6 port valve would also allow the flow to be injected directly onto the LC column as a plug of reagent, allowing the sample to be separated and passed onto the DAD for spectroscopic analysis. Chemstation (the software which controls Agilent LC systems) allows for the pumping modules, column compartment, DAD, as well as external devices such as the 6 port sampling valve, to be directly controlled on timed programmes. This would allow precise control over the flow rate, temperature and composition of the reaction, as well as the LC separation conditions during the analysis of a sample. It may also be possible to output the data from this analysis and use it as part of a control and feedback loop to define the new set of reaction conditions.

It was decided that as there was already considerable unknowns in terms of the equipment setup, the AM reactor, as well as the reaction processing parameters, that the best approach would be to work with well-known reliable chemistry. The restrictions of the SL Accura 60 material also meant that reactions must be performed in mild solvent systems, preferably at room temperature. A room temperature oxime formation in methanol was seen as a reliable 
reaction, ideal for these purposes. For all of the following initial experiments detailed, the aldehyde of choice was 4-nitrobenzaldehyde, due to the activating effect of the nitro group. This would ensure that the reaction would proceed smoothly regardless of the processing conditions.

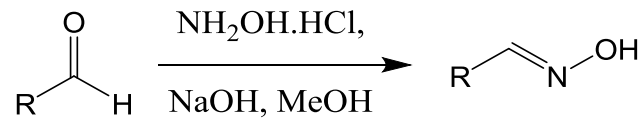

\section{Scheme 5: Reaction conditions for the oxime formation used to test SL part 7.}

The general scheme for this reaction proceeded with 4-nitrobenzaldehyde being dissolved in methanol and transferred to a sample vial. Hydroxylamine and sodium hydroxide were dissolved in water and transferred to a second sample vial. The two solutions were flowed through SL part 9 at $1.5 \mathrm{~mL} / \mathrm{min}$ giving a $\mathrm{t}_{\mathrm{R}}$ of approximately 4.8 minutes. A plug of this flow was injected onto the LC column and the data was analysed.

\section{LC analysis 1:}

This reaction was run across a range of experimental conditions, with the aldehyde to amine ratio being varied from $4: 1,3: 1,2: 1,1: 1$ and $1: 2$ respectively. The reaction and LC conditions are outlined in Table 51. Prior to this the reaction was run in batch, with the product being separated and analysed via ${ }^{1} \mathrm{H}$ NMR and IR spectroscopy. The starting material and product were then run using these LC conditions to confirm the retention times of each. The retention times for the amine starting material was approximately 2.79 minutes and the oxime product approximately 3.07 minutes. The aldehyde starting material was shown to have a maximal absorbance $(\lambda \max )$ around $265 \mathrm{~nm}$, whilst the product was shown to absorb maximally at around $350 \mathrm{~nm}$ (Figure 97). 


\begin{tabular}{|c|c|}
\hline SL Part 9 Reaction Volume & $7.2 \mathrm{~mL}$ \\
\hline Reactor Flow Rate & $1.5 \mathrm{~mL} / \mathrm{min}$ \\
\hline Residence time & 4.8 minutes \\
\hline Sample Loop Size & $3.5 \mu \mathrm{L}$ \\
\hline Column & Agilent Zorbax Eclipse Plus $4.6 \times 50$ \\
& mm um \\
\hline Column Temperature & $9: 1 \mathrm{MeOH}: \mathrm{Cater}$ \\
\hline Stationary Phase & $1 \mathrm{~mL} / \mathrm{min}$ \\
\hline Stationary Phase Flow Rate & DAD A: 260,80 nm, $2 \mathrm{~nm}$ slit width \\
\hline DAD settings & 0.066 mmol in $1000 \mathrm{~mL}$ methanol \\
\hline Aldehyde stock solution & 0.066 mmol amine, 0.066 mmol \\
\hline Amine stock solution & sodium hydroxide in 1000 mL water \\
\hline
\end{tabular}

Table 51: Reaction and LC conditions for LC analysis 1.

The retention times are slightly variable for each reaction due to the delay in the manual injection from the six port valve, however there is enough separation between the peaks to enable the easy identification of the product and the starting material. Each of the peaks was integrated giving the data set seen in Table 52.

\begin{tabular}{|c|c|c|c|}
\hline \multirow{2}{*}{$\begin{array}{c}\text { Aldehyde to } \\
\text { Amine Ratio }\end{array}$} & $\begin{array}{c}\text { Starting Material } \\
\text { Integration }\end{array}$ & $\begin{array}{c}\text { Product Integration } \\
\text { (retention time = 3.07) }\end{array}$ & $\begin{array}{c}\text { Product } \\
\text { Conversion } \\
(\%)\end{array}$ \\
\hline $\mathbf{4 : 1}$ & 4584 & 782 & 14.6 \\
\hline $\mathbf{3 : 1}$ & 3570 & 384 & 9.7 \\
\hline $\mathbf{2 : 1}$ & 2897 & 122 & 4.04 \\
\hline $\mathbf{1 : 1}$ & 1366 & 117 & 7.9 \\
\hline $\mathbf{1 : 2}$ & 4501 & 1501 & 25.0 \\
\hline
\end{tabular}

Table 52: The conversion of aldehyde to amine, during each set of conditions used in LC analysis 1.

Although the integrated peaks from the separated LC spectra clearly show that the reaction had not progressed to completion in any of the experiments, it did demonstrate that it was possible to perform a simple organic reaction within an SL manufactured reactor, whilst monitoring the reaction online via HPLC. This is also the first example of an SL 
manufactured reactor being integrated with an analytical flow system, for use in a reaction optimisation system.

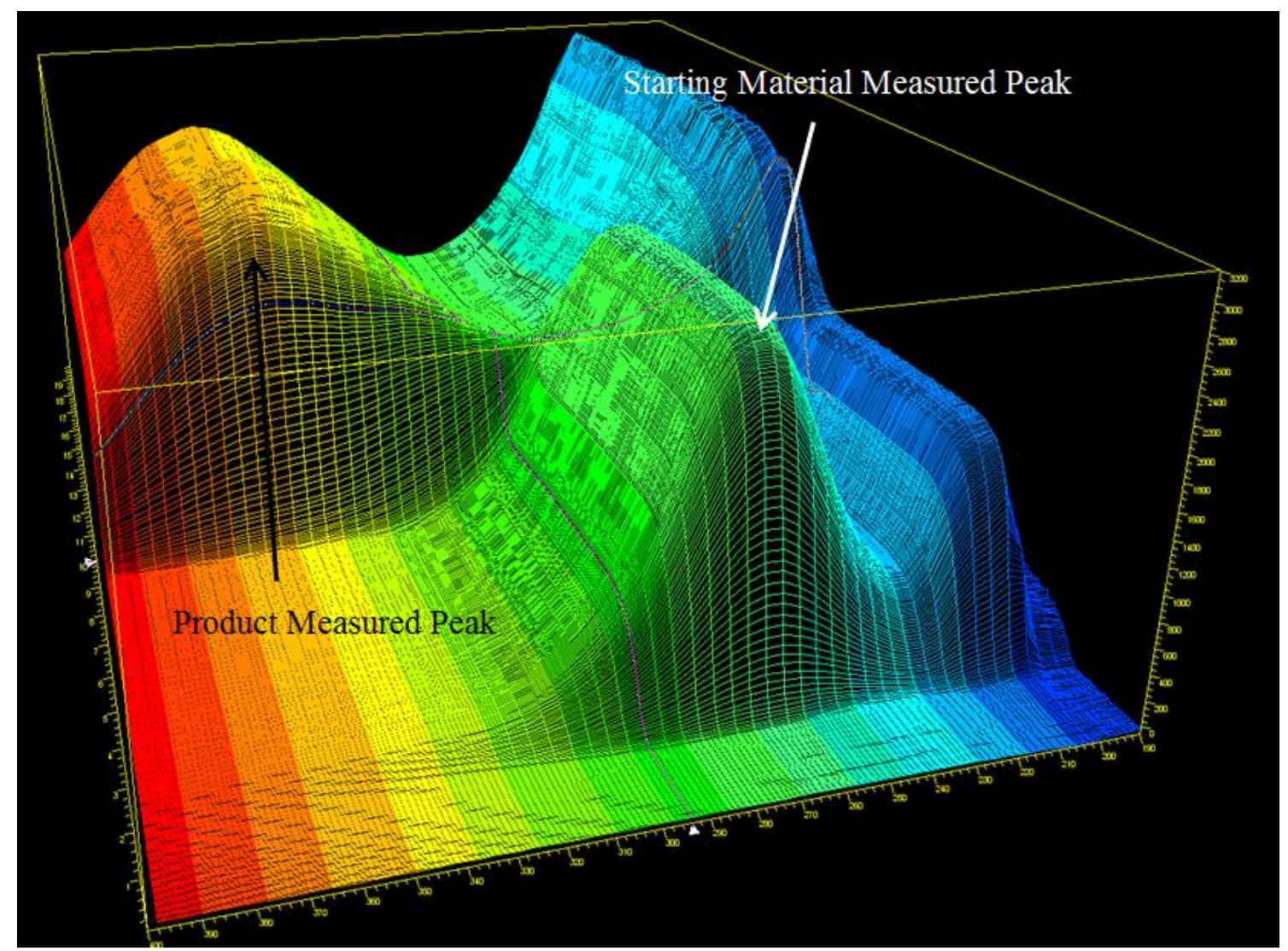

Figure 97: DAD UV-Vis Spectra for Amine Starting Material and Oxime Product. $x$-axis = wavelength $(\mathbf{n m}), \mathbf{y}$-axis $=$ absorption intensity $(\mathrm{AU}), \mathrm{z}$-axis $=$ Time $(\mathrm{mins})$

\section{LC analysis 2:}

The reaction conditions and LC analysis performed during LC analysis 1 were all manually controlled. As previously mentioned, it is possible to automate the control of these conditions using the Chemstation experimental control features. In order to test this hypothesis, the ratio of aldehyde to amine was automated to change every 10 minutes, with an aliquot of the flow stream being injected onto the column for analysis prior to this change. The concentration of aldehyde and amine within the reaction medium was also increased by an order of magnitude in order to increase the reaction rate. The reaction and LC conditions are outlined in Table 53. Conversions would be calculated from the integrated starting material and product peaks on the LC spectra. 


\begin{tabular}{|c|c|}
\hline SL Part 9 Reaction Volume & $7.2 \mathrm{~mL}$ \\
\hline Reactor Flow Rate & $1 \mathrm{~mL} / \mathrm{min}$ \\
\hline Residence time & 7.2 minutes \\
\hline Sample Loop Size & $3.5 \mu \mathrm{l}$ \\
\hline Column & $\begin{array}{c}\text { Agilent Zorbax Eclipse Plus } 4.6 \text { x } 50 \\
\text { mm x } 3.5 \text { um }\end{array}$ \\
\hline Column Temperature & $40^{\circ} \mathrm{C}$ \\
\hline Stationary Phase & 9:1 MeOH:Water \\
\hline Stationary Phase Flow Rate & $1 \mathrm{~mL} / \mathrm{min}$ \\
\hline DAD settings & $260,80 \mathrm{~nm}, 2 \mathrm{~nm}$ slit width \\
\hline Aldehyde stock solution & $0.66 \mathrm{mmol}$ in $1000 \mathrm{~mL}$ methanol \\
\hline Amine stock solution & $\begin{array}{c}0.66 \mathrm{mmol} \text { amine, } 0.66 \mathrm{mmol} \text { sodium } \\
\text { hydroxide in } 1000 \mathrm{~mL} \text { water }\end{array}$ \\
\hline
\end{tabular}

Table 53: Reaction and LC conditions for $L C$ analysis 2.

Table 54 summarises the automated reaction flow profile used during the experiment.

\begin{tabular}{|c|c|c|c|}
\hline $\begin{array}{c}\text { Time } \\
(\text { minutes })\end{array}$ & $\begin{array}{c}\text { Pump A (mL/min) - } \\
\text { Aldehyde }\end{array}$ & $\begin{array}{c}\text { Pump B (mL/min) }- \\
\text { Amine }\end{array}$ & $\begin{array}{c}\text { Conversion } \\
(\%)\end{array}$ \\
\hline $0-10$ & 1 & 0 & 0 \\
\hline $10-20$ & 0.9 & 0.1 & 1 \\
\hline $20-30$ & 0.8 & 0.2 & 8 \\
\hline $30-40$ & 0.7 & 0.3 & 49 \\
\hline $40-50$ & 0.6 & 0.4 & 51 \\
\hline $50-60$ & 0.5 & 0.5 & 83 \\
\hline $60-70$ & 0.4 & 0.6 & 100 \\
\hline $70-80$ & 0.3 & 0.7 & 37 \\
\hline
\end{tabular}

Table 54: The automated reaction flow profile used during LC analysis 2.

As the proportion of amine increased within the reaction from $0-80 \%$, the proportion of oxime product also increased until the reaction went to completion somewhere between 60 and $70 \%$ amine. 


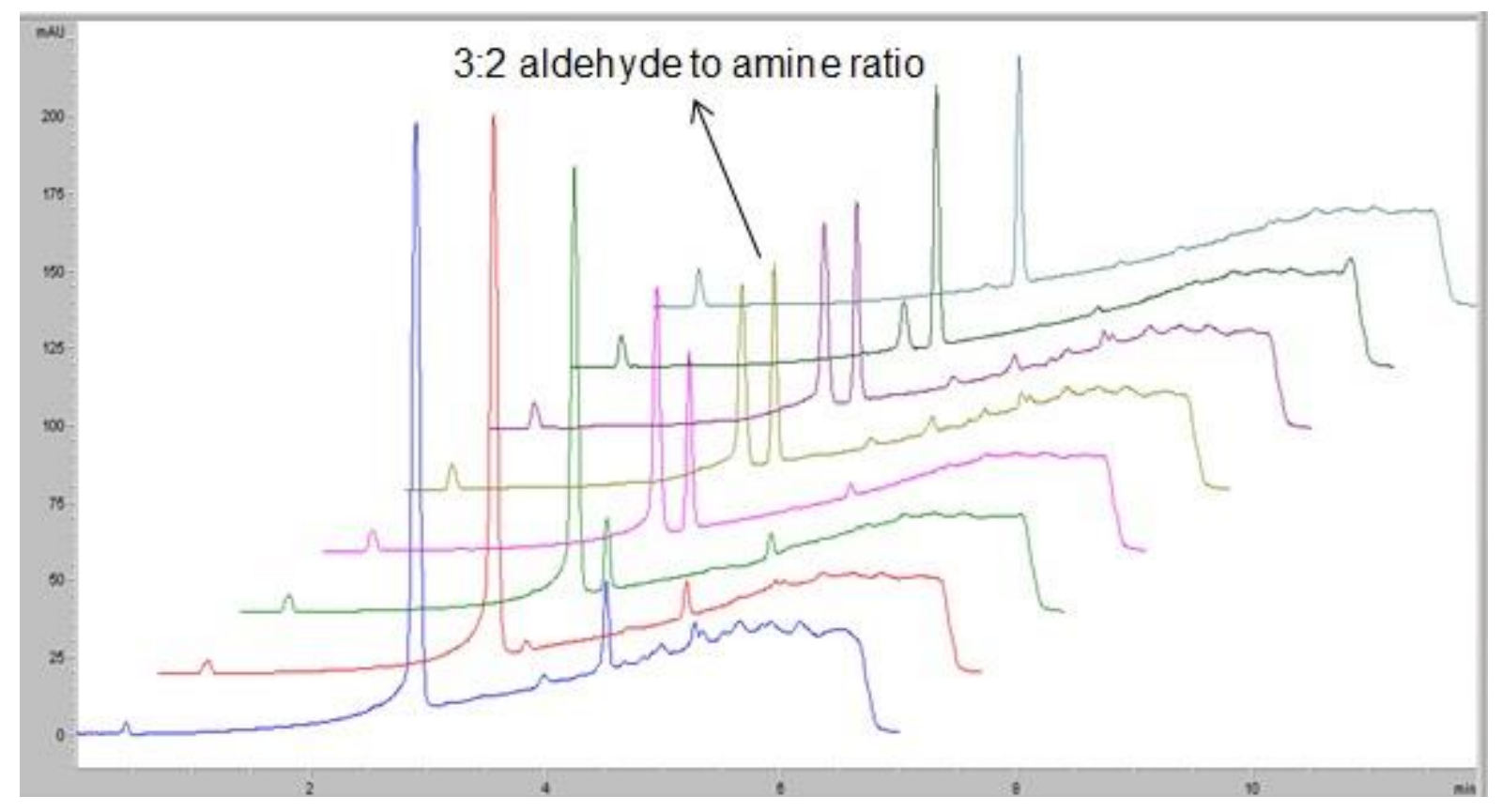

Figure 98: Chromatographic data for each of the different aldehyde to amine ratios trialled during LC analysis 2. Blue = 0-10 minutes, red $=10-20$ minutes, light green $=20-30$ minutes, pink $=30-40$ minutes, gold $=$ 40-50 minutes, purple $=\mathbf{5 0 - 6 0}$ minutes, dark green $=\mathbf{6 0 - 7 0}$ minutes, turquoise $=\mathbf{7 0 - 8 0}$ minutes .

\section{LC analysis 3:}

Having shown that it was possible to automate the ratio of each starting material reagent to optimise a reaction, the next step was to automate the overall flow rate of the reaction. The ratio of aldehyde to amine within the reaction was kept at 3:2 aldehyde to amine ratio, as this region had an even distribution of starting material and product, and therefore some scope for optimisation. Unfortunately, due to a failure in the screw thread of SL part 9, the internal reaction volume of the part to had to be reduced to $1.9 \mathrm{~mL}$ by machining a new inlet into the device. Otherwise, the reaction conditions were kept the same as those outlined in Table 53, with the specific automated flow profile outlined in Table 55.

\begin{tabular}{|c|c|c|c|c|}
\hline $\begin{array}{c}\text { Time } \\
\text { (minutes) }\end{array}$ & $\begin{array}{c}\text { Pump A (mL/min) } \\
- \text { Aldehyde }\end{array}$ & $\begin{array}{c}\text { Pump B } \\
(\mathbf{m L} / \mathbf{m i n})- \\
\text { Amine }\end{array}$ & $\begin{array}{c}\text { Residence Time } \\
(\text { minutes) }\end{array}$ & $\begin{array}{c}\text { Conversion } \\
(\%)\end{array}$ \\
\hline $0-5$ & 1.2 & 0.8 & 19 & 37 \\
\hline $5-10$ & 0.3 & 0.2 & 3.6 & 65 \\
\hline $10-20$ & 0.12 & 0.08 & 9.5 & 100 \\
\hline $20-40$ & 0.06 & 0.04 & 19 & 98 \\
\hline
\end{tabular}

Table 55: The automated reaction flow profile used during LC analysis 3. 


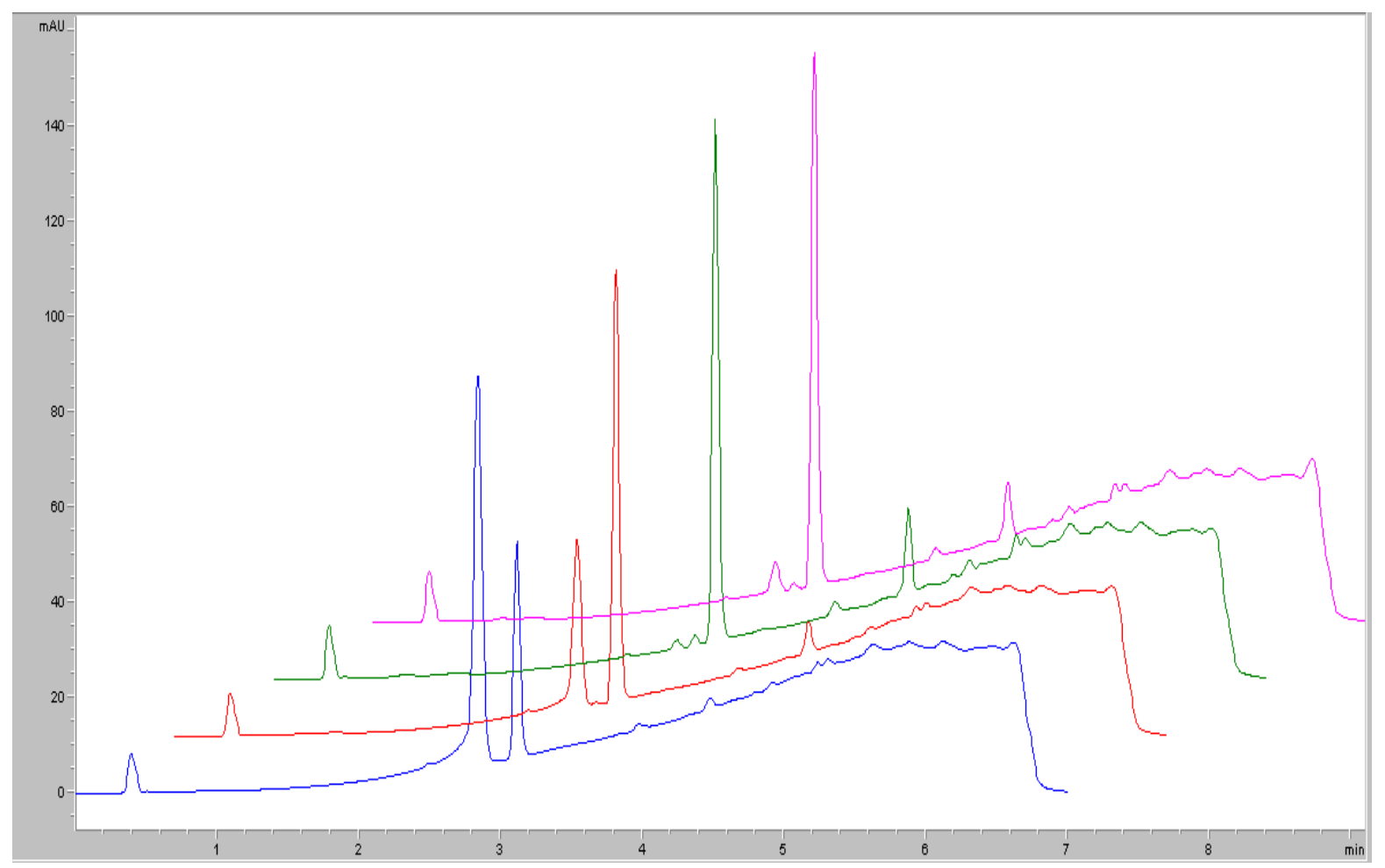

Figure 99: Chromatographic data for each of the different flow rates trialled during LC analysis 3. Blue = $2.0 \mathrm{~mL} / \mathrm{min}$, red $=0.5 \mathrm{~mL} / \mathrm{min}$, green $=0.2 \mathrm{~mL} / \mathrm{min}$, pink $=0.1 \mathrm{~mL} / \mathrm{min}$.

The results show that the reaction went to completion (>90\% conversion) at a flow rate of around $0.2 \mathrm{~mL} / \mathrm{min}$ or a residence time of around 10 minutes. No further improvements in yields were gained from reducing the flow rate further.

\section{LC analysis 4:}

Having demonstrated that it was possible to use the Agilent software to automate the reaction conditions, the next stage of the system development would be to see whether it was possible to monitor the progress of the reaction purely using the UV-Vis spectroscopy data generated from the DAD. This meant taking the reaction product flow and passing it directly through the $\mathrm{DAD}$, giving real time inline analysis of the reaction medium. This did mean that the process would not involve the separation and purification of the products on the LC column. For this reaction, the amine reagent stream was placed in excess. The reaction conditions are outlined in Table 56. 


\begin{tabular}{|c|c|}
\hline $\begin{array}{c}\text { SL Part 9 Reaction } \\
\text { Volume }\end{array}$ & $1.9 \mathrm{~mL}$ \\
\hline Reactor Flow Rate & $1 \mathrm{~mL} / \mathrm{min}$ \\
\hline Residence time & $1.9 \mathrm{mins}$ \\
\hline DAD settings & $260,80 \mathrm{~nm}, 2 \mathrm{~nm}$ slit width \\
\hline Aldehyde stock solution & $0.66 \mathrm{mmol} \mathrm{in} 1000 \mathrm{~mL}$ methanol \\
\hline Amine stock solution & $0.66 \mathrm{mmol}$ amine, $0.66 \mathrm{mmol}$ sodium hydroxide in $200 \mathrm{~mL}$ \\
& water \\
\hline
\end{tabular}

Table 56: Reaction conditions for $\mathrm{LC}$ analysis 3.

Table 57 outlines the automated flow profile of the reaction.

\begin{tabular}{|c|c|c|}
\hline Time (minutes) & Aldehyde (\%) & Amine (\%) \\
\hline 0 & 100 & 0 \\
\hline 20 & 50 & 50 \\
\hline
\end{tabular}

Table 57: Summary of the automated flow profile used during LC analysis 3.

The Chemstation software will allow visualisation of the reaction medium via a 3-D chromatogram, which shows the wavelength on the $\mathrm{x}$ axis, the time on the $\mathrm{y}$ axis and the peak intensity on the $\mathrm{z}$ axis. The 3D chromatogram from this reaction shows at a time of around 9.5 minutes, the conversion of the starting material ( $\mathrm{UV} \max =270 \mathrm{~nm}$ ) to the oxime product (UV $\max =360 \mathrm{~nm}$ ). This demonstrated that it would be possible to monitor the reaction medium of future reactions, using the DAD within the LC.

Measurements of the part were taken in the $\mathrm{x}, \mathrm{y}$ and $\mathrm{z}$ plane, comparing this data with the original CAD to determine dimensional accuracy.

\begin{tabular}{|c|c|c|c|c|}
\hline Dimension & $\begin{array}{c}\text { Actual } \\
\text { Measurement }(\mathbf{m m})\end{array}$ & $\begin{array}{c}\text { CAD measurement } \\
(\mathbf{m m})\end{array}$ & $\begin{array}{c}\text { Difference } \\
(+/-\mathbf{m m})\end{array}$ & $\begin{array}{c}\text { Variation } \\
(\boldsymbol{\%})\end{array}$ \\
\hline $\mathrm{x}$ & 100.19 & 100.00 & 0.19 & 0.19 \\
\hline $\mathrm{y}$ & 86.18 & 86.00 & 0.18 & 0.21 \\
\hline $\mathrm{z}$ & 17.52 & 17.50 & 0.02 & 0.11 \\
\hline
\end{tabular}

Table 58: Optical Calliper Measurements of SL Part 9.

A discussion about the accuracy and repeatability of all parts discussed in this section will follow later in the chapter. 


\subsubsection{SL Part 10}

\section{Design, build and post processing:}

Having demonstrated that it was possible to analyse an organic reaction by passing it directly through the HPLC DAD, it was hypothesised that it may be possible to manufacture a part that would mimic the geometrical dimensions of the Agilent flow cell, whilst incorporating a milli-scale reactor into the same device. This would allow a user to perform inline analysis of their reaction medium, without the need to break the flow path and pass into a separate part. Using this method it would be possible to produce a live data stream from the reaction, which could be utilised as part of a more advanced optimisation setup.

To begin the design process, measurements of the existing flow cell used in the HPLC DAD detector had to be taken. Typically the flow from the LC column passes along a narrow bore tube into the flow cell. The flow passes between two quartz windows which are held in place by a threaded screw cell. This enables the UV-Vis light source to pass uninterrupted through the reaction medium without disturbing the flow. The flow cell is held in place within the DAD compartment by a sprung clip, which can be attached and removed from the flow cell by two bolts. Each dimension of the flow cell was accurately measured using optical callipers, and a CAD design was produced mimicking the cell. The part was initially prototyped using FDM to optimise the design before building the part from the more expensive SL resin (Chapter 3.2.1). The design also included a space allowing the attachment of a sprung clip, to enable the part to be held in place within the DAD compartment. The UV light would pass directly across the width of the channel giving the cell a path length of $1.5 \mathrm{~mm}$. The part dimensions are outlined in Table 59. 


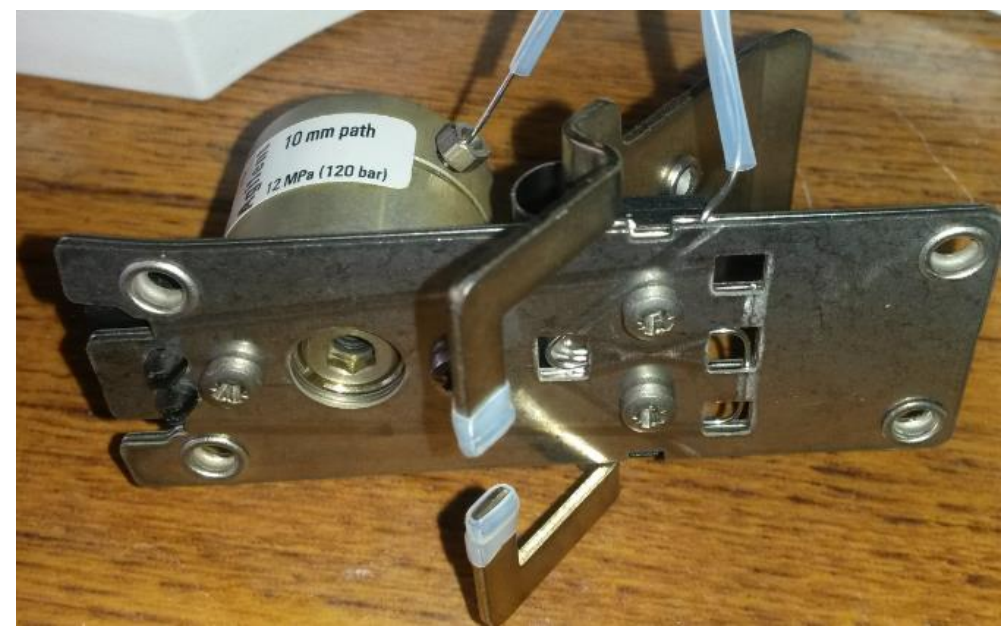

Figure 100: Original Agilent DAD Flow Cell

\begin{tabular}{|c|c|}
\hline Part Length $(\mathrm{mm})$ & 122.70 \\
\hline Part Width $(\mathrm{mm})$ & 66.50 \\
\hline Part Height $(\mathrm{mm})$ & 42.00 \\
\hline Part Volume $\left(\mathrm{cm}^{3}\right)$ & 67.70 \\
\hline Channel Diameter $(\mathrm{mm})$ & 1.50 \\
\hline Channel Length $(\mathrm{mm})$ & 1600 \\
\hline Internal Reaction Volume $\left(\mathrm{cm}^{3}\right)$ & 2.80 \\
\hline
\end{tabular}

Table 59: SL Part 10 Build Dimensions.
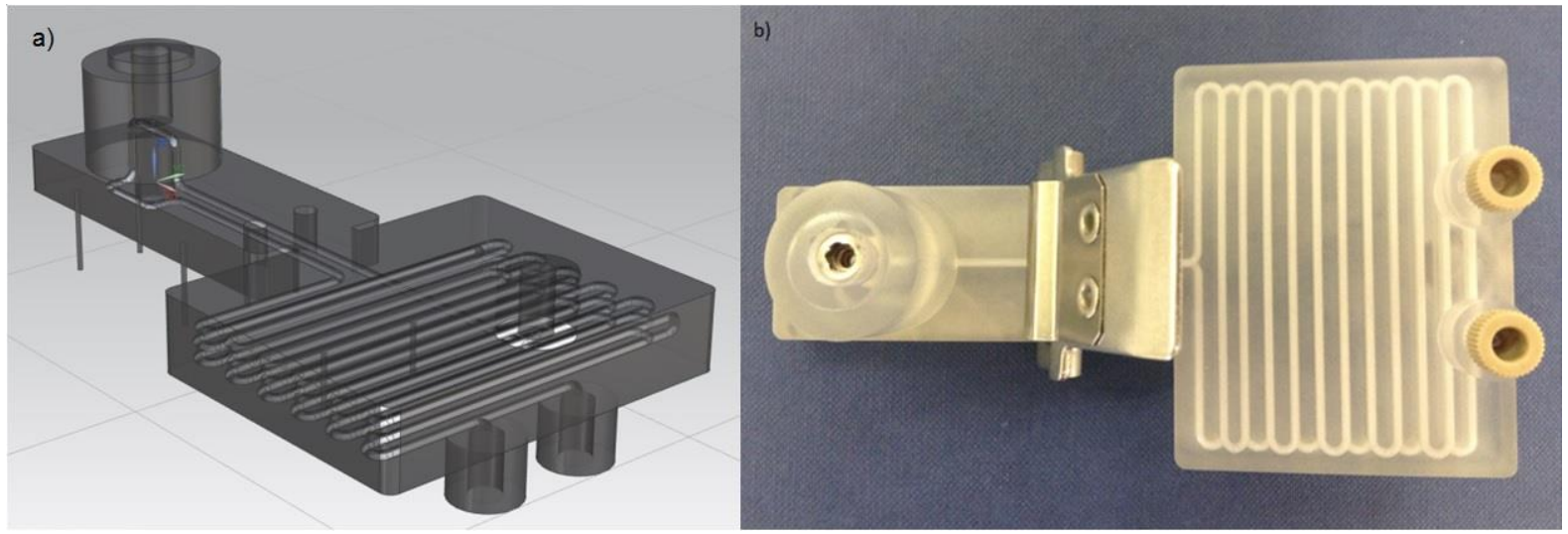

Figure 101: a) CAD design for SL part 10, b) SLA manufactured part with window inserts and sprung clip attached.

The inlet and outlet channels within the part were tapped as usual with a 1/4-28 UNF screw thread. Tapping of the quartz window ports with the M8 x 0.5 thread, although possible, often would prove problematic when using the Accura material. The fine pitch of the tap and the brittle nature of the material often led to a poor connection between the part and the quartz window. The thread would also demonstrate significant wear from repeated use of the part. It 
was therefore decided that these ports would be tapped with a much larger pitched 3/8-16 UNC thread. It was necessary to machine a new window screw which could be placed above the existing Agilent window cell. By tightening this screw, the window cell below would be forced to form a seal with the visualisation channel. The part was also fitted with an Agilent sprung clip to allow integration with DAD compartment.

\section{Application/Discussion:}

Having manufactured a part which contained inline spectroscopic capabilities, it was hypothesised that it would be possible to use it to perform more complex reaction optimisations. It was therefore necessary to develop a reaction optimisation platform which would allow complete control over reaction conditions, as well as being able to handle any data which was generated during the process. In order to do this, a series of optimisation macros and algorithms based around a simplex optimisation strategy were generated, which allowed control of the pumps, temperature compartments and analysis equipment being used. From this point forward the optimisation software and algorithms refer to those discussed in

\section{Chapter 12.1.}

In order to compare the results obtained from the optimisation runs, a model data set was generated. In order to do this a model reaction was needed. An ideal reaction was seen as being the reduction of $(R)-(-)$-carvone to its corresponding semicarbazone, using semicarbazide and sodium acetate. The reaction would run smoothly at room temperature, as well as at elevated temperatures, and mild solvent mixtures such as isopropanol, methanol, ethanol or water could be used.

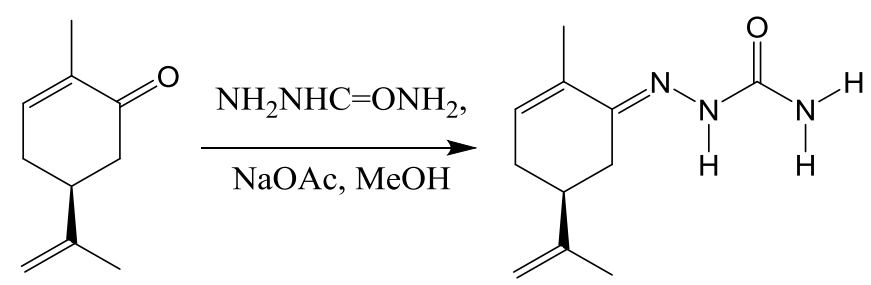

Scheme 6: Reduction of $(R)-(-)$-carvone

Below is a table outlining each of the optimisation experiments carried out, outlining the specific reaction setup and manufacturing methods used in each one. 


\begin{tabular}{|c|c|c|c|}
\hline $\begin{array}{c}\text { Optimisation } \\
\text { Number }\end{array}$ & $\begin{array}{c}\text { Reaction } \\
\text { Volume (mL) }\end{array}$ & $\begin{array}{c}\text { Reactor } \\
\text { Manufacturing } \\
\text { Process }\end{array}$ & $\begin{array}{c}\text { Analysis } \\
\text { Method Used }\end{array}$ \\
\hline Optimisation 1 & 7 & N/A & HPLC \\
\hline Optimisation 2 & 6.4 & Stereolithography & UV-Vis \\
\hline Optimisation 3 & 10 & $\begin{array}{c}\text { Selective Laser } \\
\text { Melting }\end{array}$ & HPLC \\
\hline Optimisation 4 & 11.4 & $\begin{array}{c}\text { Stereolithography } \\
\text { and Selective } \\
\text { Laser Melting }\end{array}$ & UV-Vis \\
\hline Optimisation 5 & 11.4 & $\begin{array}{c}\text { Selective Laser } \\
\text { Melting }\end{array}$ & UV-Vis \\
\hline
\end{tabular}

Table 60: Table outlining the specific reaction conditions of each optimisation run.

\section{Optimisation 1:}

Prior to this, the reaction was run in batch with the product being separated and analysed via ${ }^{1} \mathrm{H}$ NMR and IR spectroscopy. The starting material and product were then run using these LC conditions to confirm the retention times of each. The ketone starting material was shown to absorb between 200 and $270 \mathrm{~nm}$ with a maximal absorbance ( $\lambda \max )$ around $240 \mathrm{~nm}$, whilst the semicarbazide product was shown to absorb between 200 and $300 \mathrm{~nm}$ with a maximal absorbance $(\lambda \max )$ around $265 \mathrm{~nm}$.
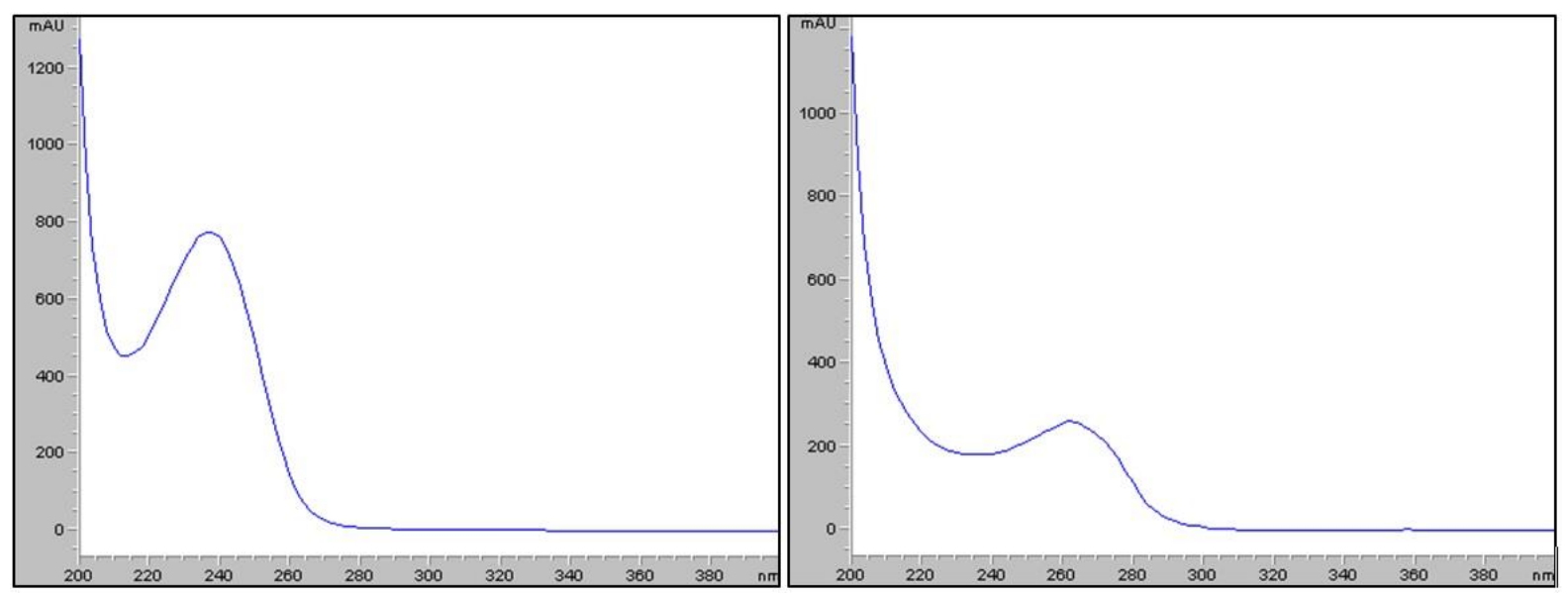

Figure 102: DAD UV-Vis Spectra for Ketone Starting Material (left) and Carbazone Product (right).

In order to generate the model data set, an 1100 series binary pump module was used to pump the two reagent flows, which passed through a $5 \mathrm{~mL}$ stainless steel coil reactor. This reactor 
was attached to a heating mantle, and heated using the temperature controlled heating module of a Uniqsis FlowSyn. This temperature would have to be manually controlled for each set of experimental conditions of the optimisation. The flow would then pass into a separate $2 \mathrm{~mL}$ stainless steel coil, which would sit in a water bath to ensure a uniform temperature of reagent. The flow would then pass into a 6 port valve, which would allow the flow to pass into either a collection vial, or be injected onto an Agilent 1100 series HPLC for data analysis (Figure 103). The analysis was set up with the conditions outlined in Table 61.

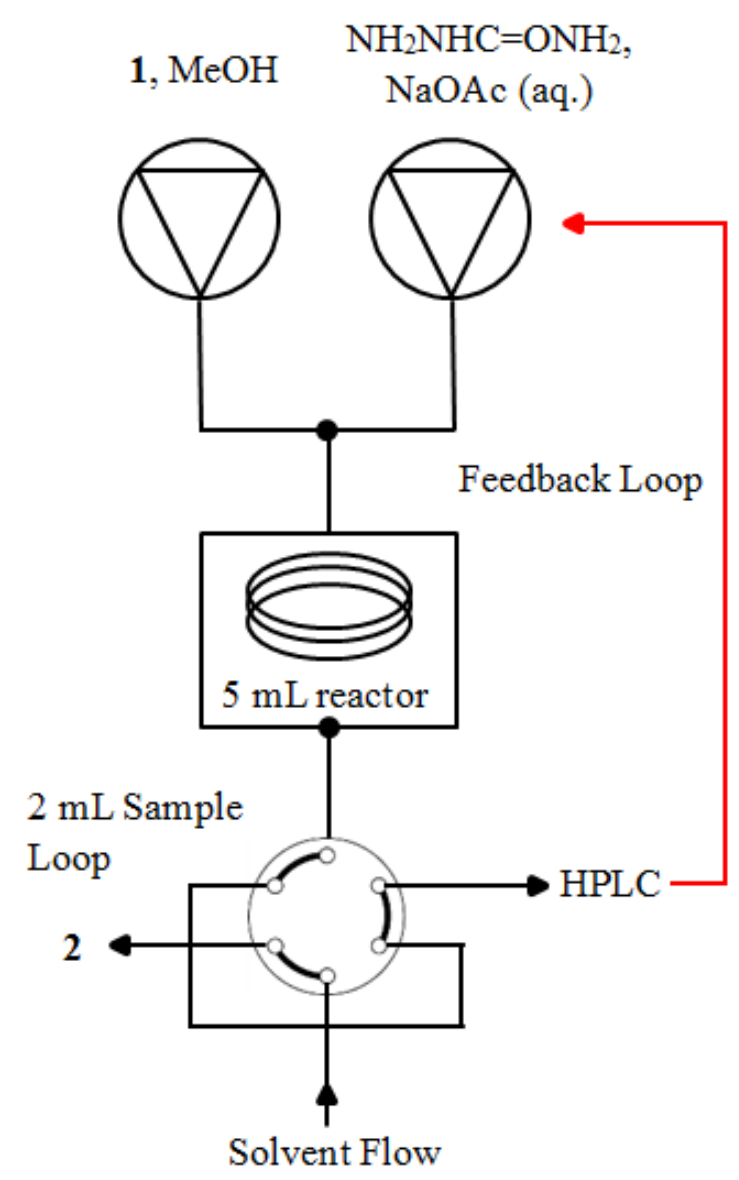

Figure 103: Schematic of the equipment setup used during optimisation 1. 


\begin{tabular}{|c|c|c|c|}
\hline Reaction Volume & \multicolumn{3}{|c|}{$7 \mathrm{~mL}$} \\
\hline DAD settings & \multicolumn{3}{|c|}{$254,16 \mathrm{~nm}, 2 \mathrm{~nm}$ slit width } \\
\hline Column & Agilent Zorbax Eclipse Plus 4.6 x 50 mmx3.5 um \\
\hline Column Temperature & \multicolumn{3}{|c|}{$30^{\circ} \mathrm{C}$} \\
\hline Mobile Phase & \multicolumn{3}{|c|}{ A: Water B:MeOH, 1 mL/min } \\
\hline \multirow{3}{*}{ Mobile Phase Gradient } & Time & A (\%) & $1 \%)$ \\
\cline { 2 - 4 } & 0 & 90 & 10 \\
\cline { 2 - 4 } & 10 & 90 & 90 \\
\cline { 2 - 4 } & 16 & 10 & 10 \\
\cline { 2 - 4 } & 17 & 90 & 50 \\
\hline Reagent Inlet A & \multicolumn{3}{|c|}{3.1 mL carvone in 2000 mL MeOH } \\
\hline Reagent Inlet B & $4.6 \mathrm{~g}$ semicarbazide, $1.7 \mathrm{~g} \mathrm{NaOAc} \mathrm{in} \mathrm{2000} \mathrm{mL} \mathrm{water}$ \\
\hline
\end{tabular}

Table 61: Reaction and analysis conditions used during optimisation 1.

The optimisation database was set up with the parameters outlined in Table 62 .

\begin{tabular}{|c|c|}
\hline Flow Rate Range & $0.2-1 \mathrm{~mL} / \mathrm{min}$ \\
\hline Temperature Range & $25-80^{\circ} \mathrm{C}$ \\
\hline Starting Temperature & $30^{\circ} \mathrm{C}$ \\
\hline Starting Flow Rate & $0.9 \mathrm{~mL} / \mathrm{min}$ \\
\hline Simplex Temperature Variation & $5^{\circ} \mathrm{C}$ \\
\hline Simplex Flow Rate Variation & $0.1 \mathrm{~mL} / \mathrm{min}$ \\
\hline Maximum Data Points & 25 \\
\hline
\end{tabular}

Table 62: Optimisation 1 database initial parameters.

The Chemstation post-run analysis macros were set up to calculate the peak area for both the carvone starting material, as well as the semicarbazone product. The macro would then output this peak data as a single \% conversion value. Due to the simplicity of this reaction, there would only be two major peaks within the spectra, so it was able to programme the software to output the peak areas for the first two data points generated. In order to prevent the software picking up any smaller peaks as a result of noise within the system, it was specified that only peaks of a height of 100 absorbance units should be considered. For more complex reactions with multiple peaks in the LC spectra, the software would need to specify individual peak retention times to be analysed. 
The optimisation was run over the period of about $20 \mathrm{~h}$, generating 25 data points within the allowable temperature and flow rate range. From this point onwards the software would run without user input, selecting the new reaction temperatures and flow rates dependent upon the prior reaction output (\% yield). This is the first literature example of an AM part being integrated with a fully automated reaction optimisation system. This determined the most optimal data point within this data set as being $75{ }^{\circ} \mathrm{C}$ and $0.25 \mathrm{~mL} / \mathrm{min}$, which had a conversion of $62.7 \%$ (Figure 104). In all likelihood the optimum data points for this reaction set would be $80{ }^{\circ} \mathrm{C}$ and $0.2 \mathrm{~mL} / \mathrm{min}$. The reason this data point was not reached was twofold. Firstly, the maximum number of data points pre-specified for the reaction meant that the optimisation finished before the entirety of the reaction space had been analysed. Secondly the size of the simplex initially selected prevented this data point from being found. A smaller simplex could have been selected, however it would have taken longer for the optimisation to have reached this conclusion. Selection of simplex size is always a factor of the accuracy of data required, as well as the amount of time allocated to each optimisation set.

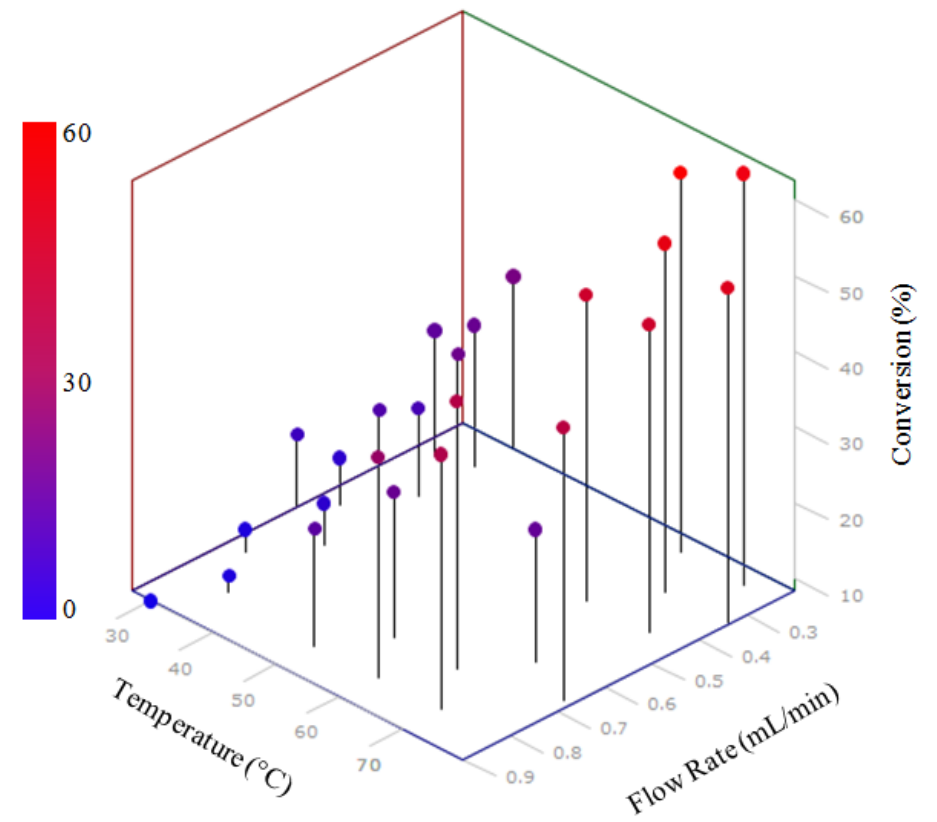

Figure 104: 3D scatter plots of optimisation 1 data set.

\section{Optimisation 2:}

Having demonstrated that the optimisation software would successfully generate a set of optimised results, the next stage in the process development would be to see if it was possible to use SL part 10 as an inline UV-Vis detector. The data generated from these UV-Vis 
spectra could then be fed back into the optimisation software. In order to do this it was necessary to create a new post-run macro (a macro which could calculate peak intensities from a UV spectra and output them as a single data value), which would be able to monitor the intensity of absorption at a single predetermined wavelength. It was decided that the absorption at $275 \mathrm{~nm}$, due to the fact that the carvone starting material did not absorb at this intensity but the semicarbazone product did. The increase in intensity of absorbance at this value could therefore be attributed to the presence of an increased concentration of the reaction product. For this optimisation an 1100 series binary pump module was used to pump our two reagent flows, which passed through a $5 \mathrm{~mL}$ stainless steel coil reactor. This reactor was attached to a heating mantle, and heated using the temperature controlled heating module of a Uniqsis FlowSyn. This temperature would have to be manually controlled for each set of experimental conditions of the optimisation. The flow would then pass into a 6 port valve, which would allow it to pass into either a collection vial, or pass through SL part 10, which had been assembled and placed within the DAD compartment of an Agilent 1100 HPLC. Although SL part 10 had an internal reaction volume of $2.8 \mathrm{~mL}$, the analysis point was situated half way along this flow path, meaning that the data being generated had effectively passed through a reaction volume of $1.4 \mathrm{~mL}$. It was also necessary to use a much more dilute reagent stream than previously used to prevent saturation of the DAD. This was due to the lack of any LC analysis which would effectively dilute our reagent stream before it reached the detector (Figure 105). The analysis was set up with the conditions outlined in Table 63. 


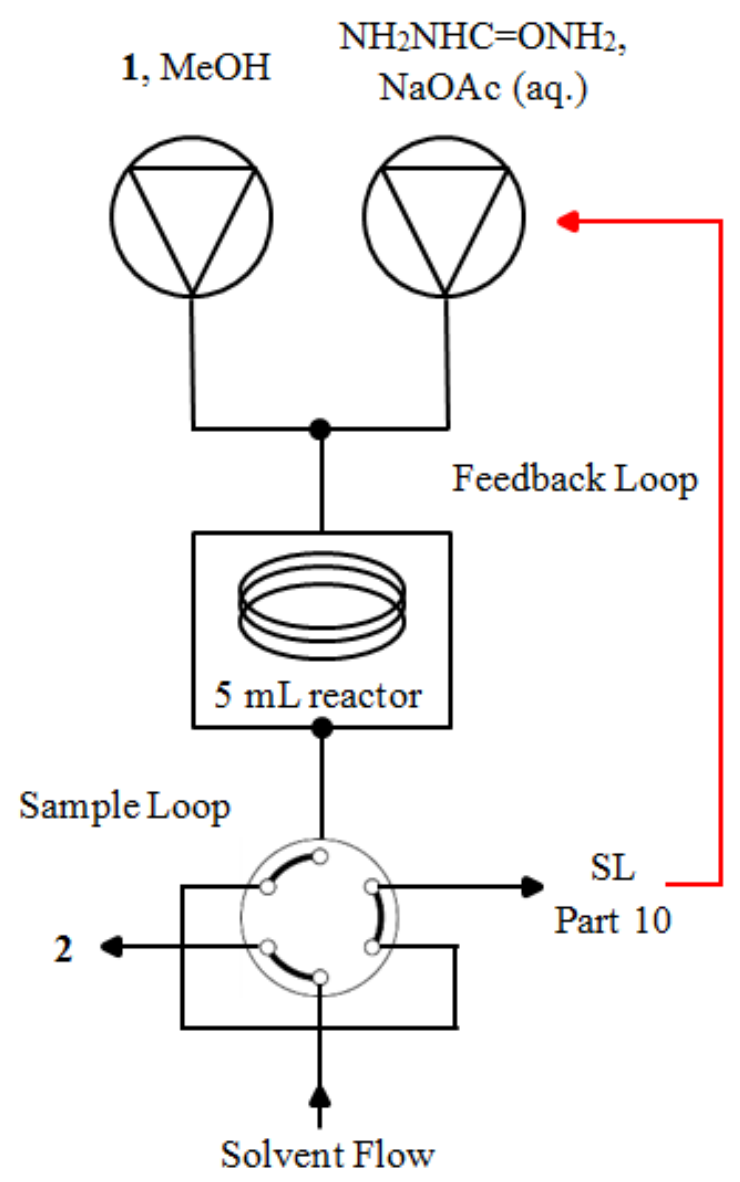

Figure 105: Schematic of the equipment setup used during optimisation 2.

\begin{tabular}{|c|c|}
\hline Reaction Volume & $6.4 \mathrm{~mL}$ \\
\hline DAD settings & $275,4 \mathrm{~nm}, 2 \mathrm{~nm}$ slit width \\
\hline Reagent Inlet A & $0.124 \mathrm{~mL}$ carvone in $2000 \mathrm{~mL} \mathrm{MeOH}$ \\
\hline Reagent Inlet B & $0.184 \mathrm{~g}$ semicarbazide, $0.068 \mathrm{~g} \mathrm{NaOAc}$ in $2000 \mathrm{~mL}$ water \\
\hline
\end{tabular}

Table 63: Reaction and analysis conditions used during optimisation 2.

The optimisation database was set up with the parameters outlined in Table 64 . 


\begin{tabular}{|c|c|}
\hline Flow Rate Range & $0.2-1 \mathrm{~mL} / \mathrm{min}$ \\
\hline Temperature Range & $25-80^{\circ} \mathrm{C}$ \\
\hline Staring Temperature & $40^{\circ} \mathrm{C}$ \\
\hline Starting Flow Rate & $0.8 \mathrm{~mL} / \mathrm{min}$ \\
\hline Simplex Temperature Variation & $5^{\circ} \mathrm{C}$ \\
\hline Simplex Flow Rate Variation & $0.1 \mathrm{~mL} / \mathrm{min}$ \\
\hline Maximum Data Points & 50 \\
\hline
\end{tabular}

Table 64: Optimisation 2 database initial parameters.

In order to avoid any signal noise from the solvent, the DAD detector was balanced (the current spectra of the flow cell was taken and set as the baseline reading) before analysis against a 50:50 MeOH:water mixture. Due to lack of any LC analysis for each iteration of the optimisation, the total run time would be significantly reduced, therefore it was possible to generate twice as many data points within a similar time frame. The optimisation was run over the period of about $13 \mathrm{~h}$, generating 50 data points within the allowable temperature and flow rate range. This determined the most optimal data point within this data set as being $69.4^{\circ} \mathrm{C}$ and $0.27 \mathrm{~mL} / \mathrm{min}$ (Figure 106).

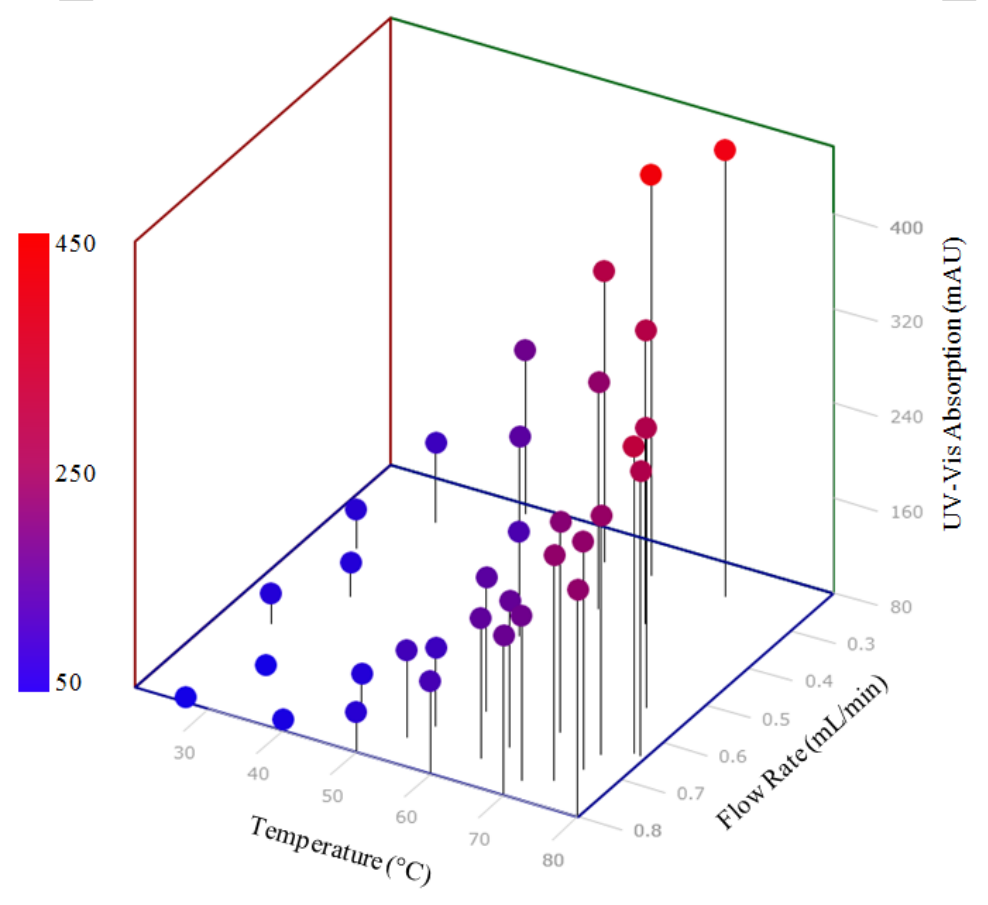

Figure 106: 3D scatter plot of optimisation 2 data set.

Without the LC analysis to dilute the reagent flow, the reaction medium had to be diluted to a point where both reaction conversion, and also total product production rate, had been 
considerably reduced from previous runs. Future work needs to be undertaken to allow dilution of the reagent stream to be carried out after the reaction has gone to completion, either via an external device or within the body of the reactor itself.

Measurements of the part were taken in the $\mathrm{x}, \mathrm{y}$ and $\mathrm{z}$ plane, comparing this data with the original CAD to determine dimensional accuracy.

\begin{tabular}{|c|c|c|c|c|}
\hline Dimension & $\begin{array}{c}\text { Actual } \\
\text { Measurement }(\mathbf{m m})\end{array}$ & $\begin{array}{c}\text { CAD measurement } \\
(\mathbf{m m})\end{array}$ & $\begin{array}{c}\text { Difference } \\
(+/-\mathbf{m m})\end{array}$ & $\begin{array}{c}\text { Variation } \\
(\boldsymbol{\%})\end{array}$ \\
\hline $\mathrm{x}$ & 66.98 & 66.50 & 0.48 & 0.72 \\
\hline $\mathrm{y}$ & 53.75 & 53.49 & 0.26 & 0.48 \\
\hline $\mathrm{z}$ & 13.53 & 13.33 & 0.20 & 1.50 \\
\hline
\end{tabular}

Table 65: Optical Calliper Measurements of SL Part 10.

\subsubsection{SLM Part 8}

\section{Design, build and post processing:}

In previous optimisations, a $5 \mathrm{~mL}$ stainless steel reactor coil was used in tandem with a FlowSyn heating mantle to heat and cool the reaction medium, before the inline reaction analysis step. It was hypothesised that it would be possible to use the inbuilt heated column compartment within an Agilent LC system to heat and cool our reaction medium. To do this a thermally conductive flow reactor would need to be manufactured, which mirrored the dimensions of this compartment. In order to do this, measurements of the internal dimensions of the column compartment were taken, with the external measurements of SLM part 8 mirroring them. The part had a simple channel passing through the part. The part was therefore manufactured from 316L stainless steel using the Realizer SLM 50, with the dimensions outlined in Table 66. 


\begin{tabular}{|l|l|}
\hline Part Length (mm) & 100.00 \\
\hline Part Width (mm) & 20.00 \\
\hline Part Height (mm) & 20.00 \\
\hline Part Volume $\left(\mathrm{cm}^{3}\right)$ & 31.60 \\
\hline Channel Diameter $(\mathrm{mm})$ & 2.00 \\
\hline Channel Length (mm) & 3200 \\
\hline Internal Reaction Volume $\left(\mathrm{cm}^{3}\right)$ & 10.05 \\
\hline Path Length (mm) & 4.00 \\
\hline
\end{tabular}

Table 66: SLM Part 9 Build Dimensions

By manufacturing the part from stainless steel and with an internal reaction volume of $10 \mathrm{~mL}$, SLM part 8 should be an ideal replacement for any commercially available reactor coil. The reactor was tapped at each end using a 10-32 UNF screw thread, and a PTFE o-Ring was inserted into both ports. Powder removal for the part proved incredibly difficult, probably due to the fact the channel was over $3 \mathrm{~m}$ in length. However, repeated cycles of placing the part in an ultrasonic path to agitate the powder, oven heating the part to dry the powder out, and firing a compressed air line down the channels, eventually cleared the part of all non-melted powder.

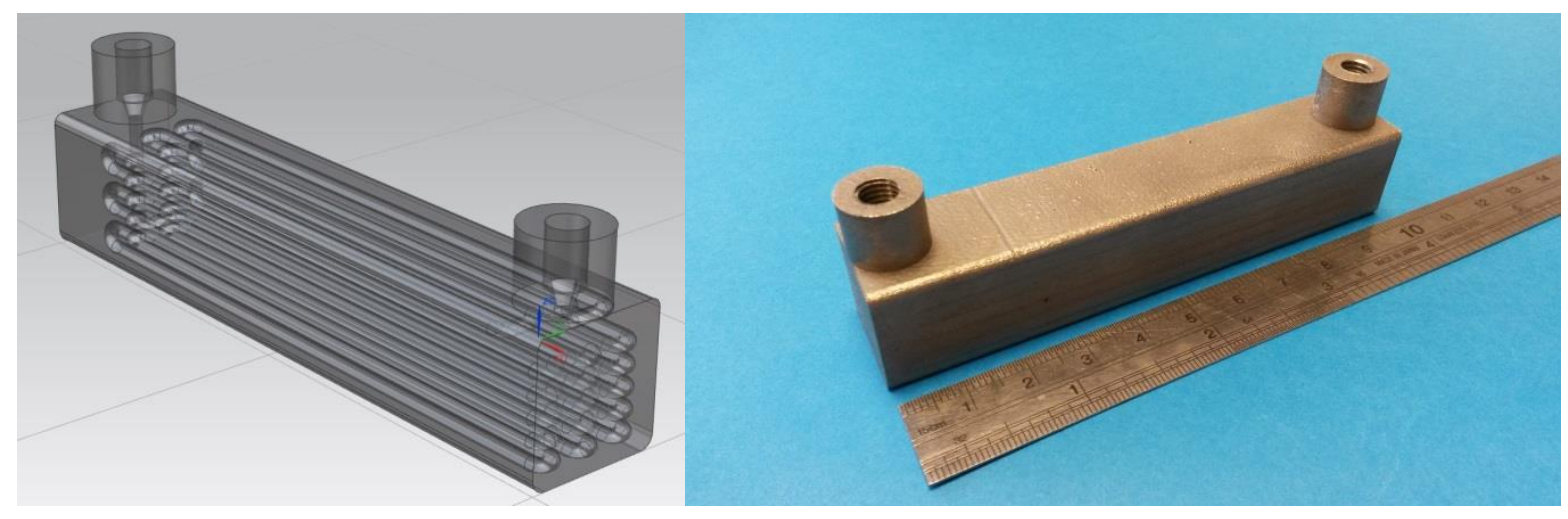

Figure 107: CAD image of SLM Part 8.

\section{Application/Discussion:}

\section{Optimisation 3:}

For this optimisation, an 1100 series binary module was used to pump our two reagent flows, which passed through SLM part 8. This reactor was placed into the LC column compartment, 
and heated using the temperature control settings within the Chemstation software. The flow would then pass into a 6 port valve, which would allow the flow to pass into either a collection vial, or be injected onto an Agilent 1100 series HPLC for data analysis (Figure 108). The analysis was set up with the conditions outlined in Table 67.

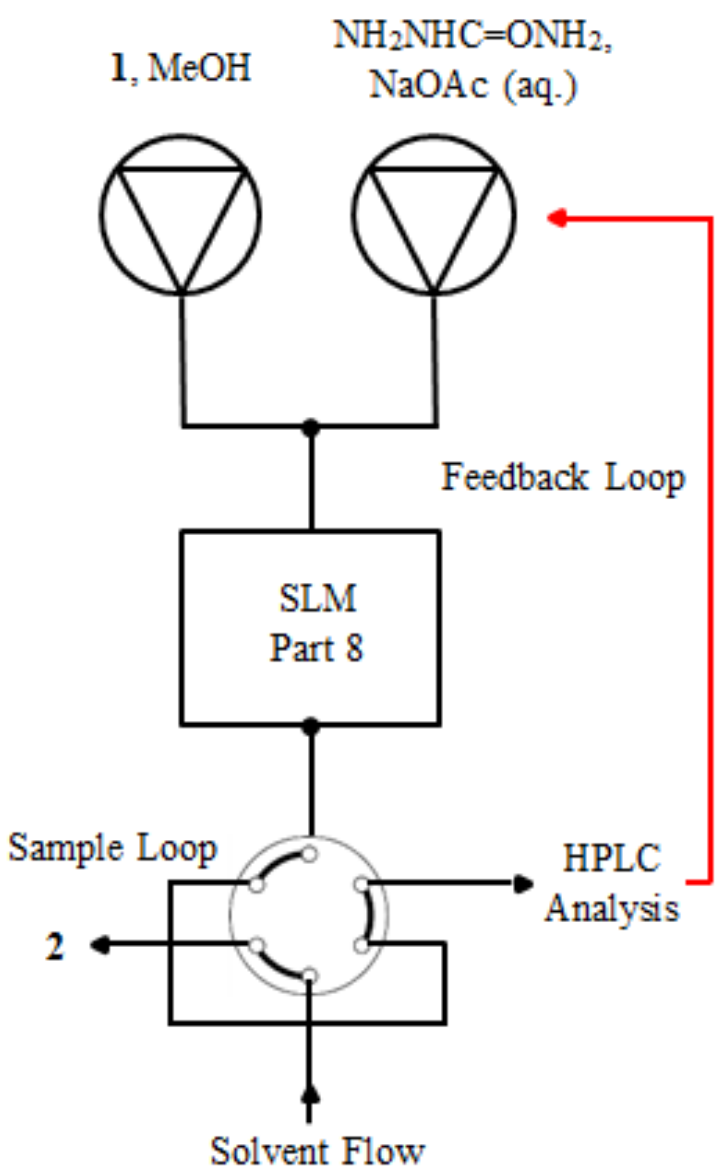

Figure 108: Schematic of the equipment setup used during optimisation 3. 


\begin{tabular}{|c|c|c|c|}
\hline Reaction Volume & \multicolumn{3}{|c|}{$10.05 \mathrm{~mL}$} \\
\hline DAD settings & \multicolumn{3}{|c|}{$254,16 \mathrm{~nm}, 2 \mathrm{~nm}$ slit width } \\
\hline Column & Agilent Zorbax Eclipse Plus 4.6 x 50 mmx3.5 um \\
\hline Column Temperature & \multicolumn{3}{|c|}{$30^{\circ} \mathrm{C}$} \\
\hline Mobile Phase & \multicolumn{3}{|c|}{ A: Water B:MeOH, 1 mL/min } \\
\hline \multirow{3}{*}{ Mobile Phase Gradient } & Time & A (\%) & 10 \\
\cline { 2 - 4 } & 0 & 90 & 10 \\
\cline { 2 - 4 } & 10 & 90 & 90 \\
\cline { 2 - 4 } & 16 & 10 & 10 \\
\cline { 2 - 4 } & 17 & 90 & 50 \\
\hline Reagent Inlet A & \multicolumn{3}{|c|}{3.1 mL carvone in 2000 mL MeOH } \\
\hline Reagent Inlet B & 4.6 g semicarbazide, $1.7 \mathrm{~g} \mathrm{NaOAc} \mathrm{in} \mathrm{2000} \mathrm{mL} \mathrm{water}$ \\
\hline
\end{tabular}

Table 67: Reaction and analysis conditions used during optimisation 3.

The optimisation database was set up with the parameters outlined in Table 68 .

\begin{tabular}{|c|c|}
\hline Flow Rate Range & $0.2-1 \mathrm{~mL} / \mathrm{min}$ \\
\hline Temperature Range & $25-80^{\circ} \mathrm{C}$ \\
\hline Staring Temperature & $40^{\circ} \mathrm{C}$ \\
\hline Starting Flow Rate & $0.8 \mathrm{~mL} / \mathrm{min}$ \\
\hline Simplex Temperature Variation & $5^{\circ} \mathrm{C}$ \\
\hline Simplex Flow Rate Variation & $0.1 \mathrm{~mL} / \mathrm{min}$ \\
\hline Maximum Data Points & 50 \\
\hline
\end{tabular}

Table 68: Optimisation 3 database initial parameters.

Again the Chemstation post-run analysis macros were set up to calculate the peak area for both the carvone starting material, as well as the semicarbazone product, outputted as a single $\%$ conversion value. The optimisation was run over the period of about $30 \mathrm{~h}$, generating 41 data points within our allowable temperature and flow rate range. This determined the most optimal data point within this data set as being $79.6^{\circ} \mathrm{C}$ and $0.24 \mathrm{~mL} / \mathrm{min}$, which had a conversion of $56.1 \%$. 


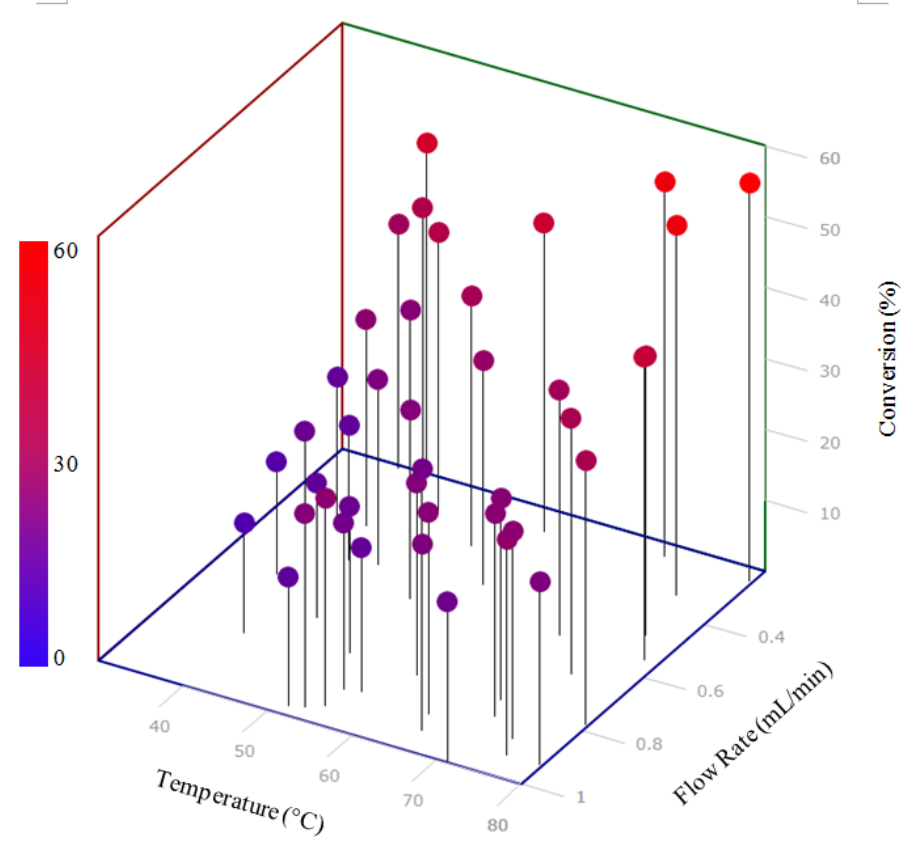

Figure 109: 3D scatter plot of optimisation 3 data set.

At present, the Chemstation macros will not begin the next reaction analysis period until the initial reaction conditions are met (equilibration of flow rate and temperature). This is most pertinent when the reaction temperature changes significantly between runs. This temperature is monitored by the inbuilt thermocouples within the LC column compartment. However, this temperature will invariably not be the same as the actual temperature of the reactor itself, and will lead to reactions conditions being run at temperatures near, but not identical, to the desired conditions. This could be overcome by embedding a thermocouple directly into the reactor itself, and using this temperature, rather than the column compartment temperature to determine when the initial reaction conditions have been met. This topic is discussed further during chapter 10. This is believed to be the reason why total conversion for the reaction optimum is slightly lower than that observed during optimisation 1, despite the fact that total residence time had been increased. It is believed that the actual average temperature across the reactor was lower than the reading given out by the LC column compartment, leading to a reduction in total conversion.

\section{Optimisation 4:}

The next logical stage in the optimisation development work would be to see whether the same optimisation using both SL part 10 and SLM part 8 could be performed at the same time. For this optimisation an 1100 series binary pump module was used to pump our two 
reagent flows, which passed through SLM part 8. This reactor was placed into the LC column compartment, and heated using the temperature control settings within the Chemstation software. The flow would then pass into SL part 10 which would sit within the DAD compartment (Figure 110). Again, the reaction medium would need to be diluted by an order of magnitude, to avoid overloading the DAD.

The analysis was set up with the conditions outlined in Table 69.

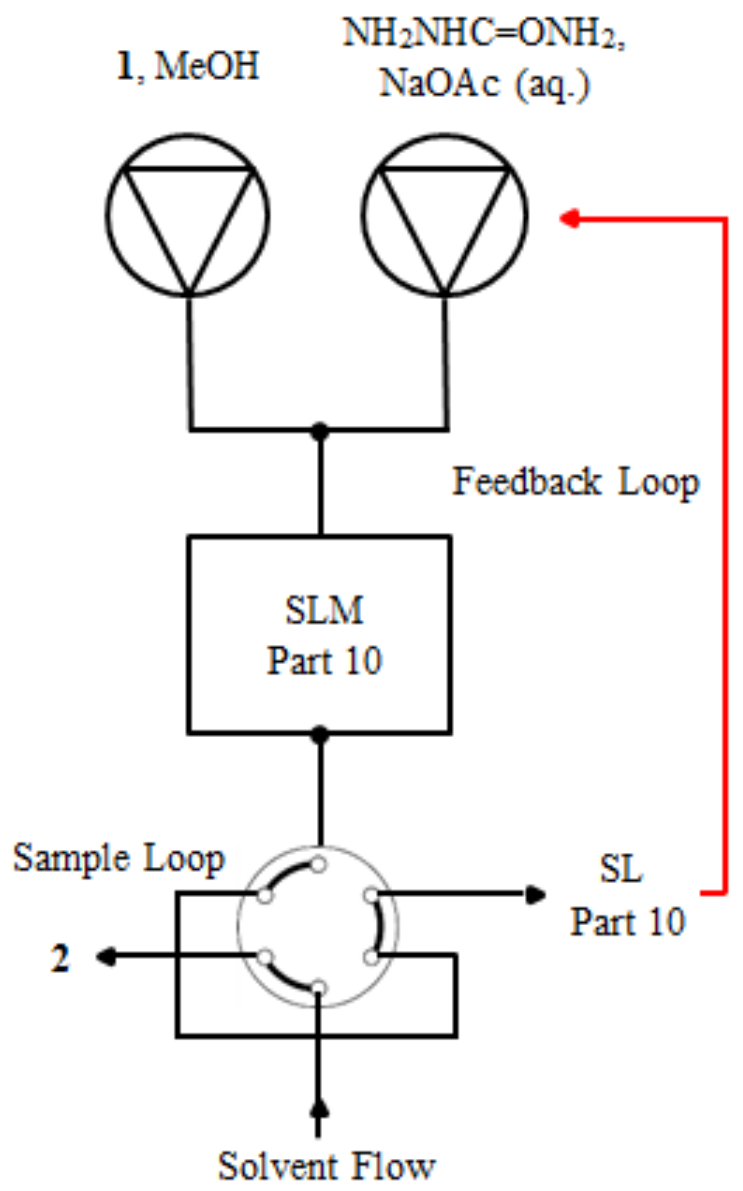

Figure 110: Schematic of the equipment setup used during optimisation 4 .

\begin{tabular}{|c|c|}
\hline Reaction Volume & $11.25 \mathrm{~mL}$ \\
\hline DAD settings & $254,16 \mathrm{~nm}, 2 \mathrm{~nm}$ slit width \\
\hline Reagent Inlet A & $0.124 \mathrm{~mL}$ carvone in $2000 \mathrm{~mL} \mathrm{MeOH}$ \\
\hline Reagent Inlet B & $0.184 \mathrm{~g}$ semicarbazide, $0.068 \mathrm{~g} \mathrm{NaOAc}$ in $2000 \mathrm{~mL}$ water \\
\hline
\end{tabular}

Table 69: Reaction and analysis conditions used during optimisation 4.

The optimisation database was set up with the parameters outlined in table 69 . 


\begin{tabular}{|c|c|}
\hline Flow Rate Range & $0.2-1 \mathrm{~mL} / \mathrm{min}$ \\
\hline Temperature Range & $25-80^{\circ} \mathrm{C}$ \\
\hline Staring Temperature & $60^{\circ} \mathrm{C}$ \\
\hline Starting Flow Rate & $0.4 \mathrm{~mL} / \mathrm{min}$ \\
\hline Simplex Temperature Variation & $5^{\circ} \mathrm{C}$ \\
\hline Simplex Flow Rate Variation & $0.1 \mathrm{~mL} / \mathrm{min}$ \\
\hline Maximum Data Points & 25 \\
\hline
\end{tabular}

Table 70: Optimisation 4 database initial parameters.

Now that the optimum data point had been determined as being $80^{\circ} \mathrm{C}$ and $0.2 \mathrm{~mL} / \mathrm{min}$, the initial starting flow rates and temperatures could be adjusted to nearer the centre of the plot. This should mean that the optimum conditions are reached in fewer data points, and a shorter time period. The optimisation was run over the period of about 7 hours, generating 25 data points within the allowable temperature and flow rate range. This determined the most optimal data point within this data set as being $70^{\circ} \mathrm{C}$ and $0.2 \mathrm{~mL} / \mathrm{min}$.

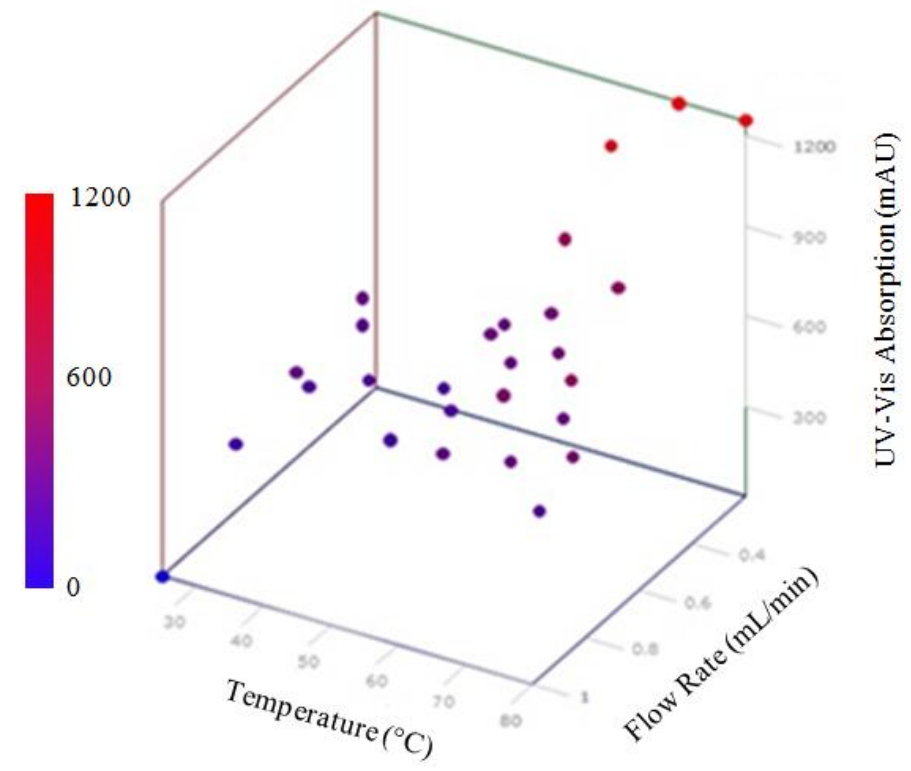

Figure 111: 3D scatter plot of optimisation 4 data set.

The absorption intensity for this data set is much higher than the values calculated during optimisation 2. The Agilent flow cell actually has a $6 \mathrm{~mm}$ flow path, compared to the $4 \mathrm{~mm}$ flow path of SL part 10. It would therefore be expected that absorption values would be lower for this part, however the flow during this optimisation passed directly through the flow cell, 
whereas during optimisation 2 the flow was diluted by being injected onto a separate flow stream via a 6 port injection valve.

Measurements of the part were taken in the $\mathrm{x}$ and $\mathrm{y}$, comparing this data with the original CAD to determine dimensional accuracy.

\begin{tabular}{|c|c|c|c|c|}
\hline Dimension & $\begin{array}{c}\text { Actual } \\
\text { Measurement }(\mathbf{m m})\end{array}$ & $\begin{array}{c}\text { CAD measurement } \\
(\mathbf{m m})\end{array}$ & $\begin{array}{c}\text { Difference } \\
(+/-\mathbf{m m})\end{array}$ & $\begin{array}{c}\text { Variation } \\
(\boldsymbol{\%})\end{array}$ \\
\hline $\mathrm{x}$ & 11.90 & 12.00 & 0.10 & 0.84 \\
\hline $\mathrm{y}$ & 19.92 & 20.00 & 0.08 & 0.40 \\
\hline
\end{tabular}

Table 71: Optical Calliper Measurements of SLM Part 8.

\subsubsection{SLM Part 9}

\section{Design, build and post processing:}

Having successfully demonstrated that it was possible to manufacture a flow cell for UV-Vis spectroscopic analysis via the SL process, the next logical stage of development would be to produce a similar part from metal. This would allow high temperature reactions in a much larger range of chemical reagent and solvents to be performed. The design for SLM part 9 was based on the previous design of SL part 10, however the large tube network attached to the quartz window viewing port was removed. This was not only to reduce the time required to produce the build, as well as the cost, but also to prevent any issues with powder removal from compromising the success of the build. SLM part 9 was therefore manufactured from 316L stainless steel using the Realizer SLM 50, with the dimensions outlined in Table 72.

\begin{tabular}{|l|l|}
\hline Part Length $(\mathrm{cm})$ & 89.00 \\
\hline Part Width $(\mathrm{cm})$ & 26.50 \\
\hline Part Height $(\mathrm{cm})$ & 38.00 \\
\hline Part Volume $\left(\mathrm{cm}^{3}\right)$ & 24.60 \\
\hline Channel Diameter $(\mathrm{mm})$ & 2.00 \\
\hline Channel Length $(\mathrm{mm})$ & 191.00 \\
\hline Internal Reaction Volume $\left(\mathrm{cm}^{3}\right)$ & 0.60 \\
\hline Path Length $(\mathrm{mm})$ & 2.00 \\
\hline
\end{tabular}

Table 72: SLM Part 9 Build Dimensions 
The reactor was tapped at each end using a 10-32 UNF screw thread, and a PTFE o-Ring was inserted into both ports. The quartz window ports were tapped with a 3/8-16 UNC thread, and secured in place with one of our previously manufactured window screws. A sprung clip was also secured to the part using two threaded bolts.

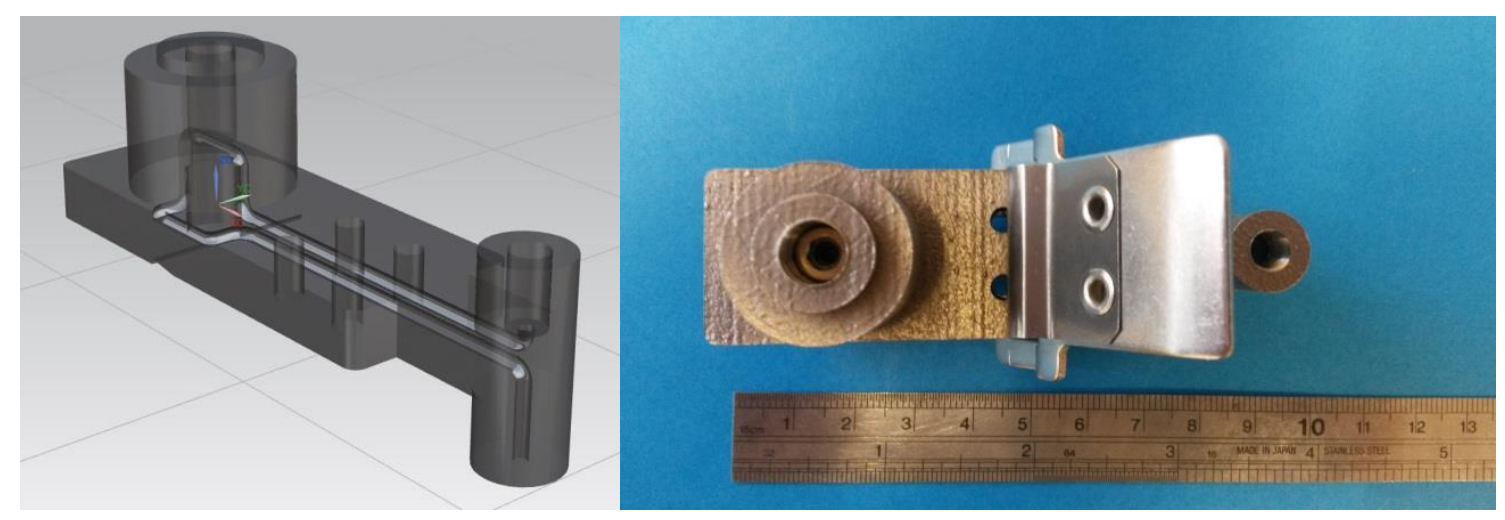

Figure 112: CAD Model of SLM Part 9.

\section{Application/Discussion:}

\section{Optimisation 5:}

The reaction and analysis conditions from optimisation 4 were repeated, except that SLM part 9 was placed into the DAD compartment instead of SL part 10. This reduced the internal reaction volume to $10.3 \mathrm{~mL}$. The optimisation was run over the period of about 6 hours, generating 20 data points within the allowable temperature and flow rate range. This determined the most optimal data point within this data set as being $75^{\circ} \mathrm{C}$ and $0.2 \mathrm{~mL} / \mathrm{min}$. 


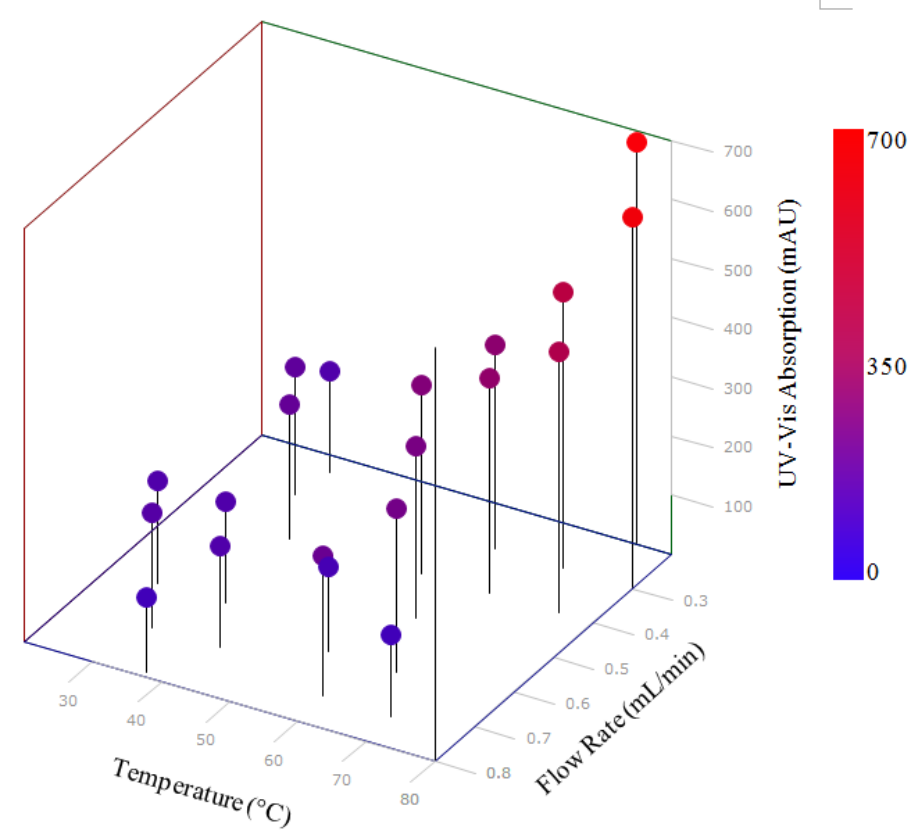

Figure 113: 3D scatter plot of optimisation 5 data set.

The $2 \mathrm{~mm}$ path length of SLM part 2, is exactly half the path length of the previously used SL part 10. As path length and absorption are directly proportional, then comparable data points should be exactly half the value of those seen during optimisation 4. By and large this is true, with the maximal absorption of $680 \mathrm{mAU}$ being approximately half the maximal absorption of $1254 \mathrm{mAU}$ seen in optimisation 4. However, it should be noted that the data values obtained during both optimisation 4 and 5 were nowhere near as reproducible as those produced when using the Agilent flow cell in optimisation 2 (e.g. occasional blank readings caused by air bubbles within the system caused the data set to fail). The Agilent flow cell is a highly optimised and precise instrument that as of yet these designs have been unable to repeat. Future iterations of this design type will be able to build on the progress made during this research, hopefully allowing the manufacture analytical instruments capable of analysing reaction mediums to a higher degree of accuracy.

Measurements of the part were taken in the $\mathrm{x}$ and $\mathrm{y}$ plane, comparing this data with the original CAD to determine dimensional accuracy.

\begin{tabular}{|c|c|c|c|c|}
\hline Dimension & $\begin{array}{c}\text { Actual } \\
\text { Measurement }(\mathbf{m m})\end{array}$ & $\begin{array}{c}\text { CAD measurement } \\
(\mathbf{m m})\end{array}$ & $\begin{array}{c}\text { Difference } \\
(+/-\mathbf{m m})\end{array}$ & $\begin{array}{c}\text { Variation } \\
(\mathbf{\%})\end{array}$ \\
\hline $\mathrm{x}$ & 26.37 & 26.46 & 0.09 & 0.34 \\
\hline $\mathrm{y}$ & 23.81 & 24.00 & 0.19 & 0.80 \\
\hline
\end{tabular}

Table 73: Optical Calliper Measurements of SLM Part 9. 
In general, dimensional accuracy of parts built via the SLM process using the Realizer SLM 50 system were good. Of the two parts manufactured, dimensions were on average within 142 $\mu \mathrm{m}$ of the intended dimensions. This equated to around a $0.69 \%$ discrepancy in geometrical accuracy.

\subsection{Conclusion}

This research has only really scratched the surface of the range of analytical devices that can be integrated into a fluidic device via AM processes. Looking towards the future, manufacturing reactors which are capable of multiple types of inline analysis would constitute a significant leap forward for continuous flow processes. Combining this technology with existing research into reaction optimisation and automation would give these flow devices considerable added benefits over existing micro and milli scale reactors.

\section{4. $\quad$ Future Work}

Future work will focus on optimisation of the inline spectroscopic analysis of reaction mediums. This will include looking at different analytical measurements such as IR and fluorescence spectroscopy. Other work will be focussed on non-spectroscopic analytical techniques such as mass spectrometry, and how AM can be used to easily integrate existing flow chemistry equipment with a wide range of analytical techniques. As well as this, the potential manufacturing of a range of parts which can be integrated to existing flow chemistry equipment as well as general laboratory pumping technologies. This should hopefully lead to an increased functionality of existing flow devices, as well as reduce the cost and time associated with producing this type of reactor. Finally, continuous development of reaction optimisation software that can be easily integrated to the diverse range of analytical data being produced by the devices. This software should be user-friendly and customisable allowing simplistic integration between different flow chemistry setups. 


\section{8. $\quad$ Ultrasonic Additive Manufacture}

\subsection{Introduction}

Ultrasonic Additive Manufacture (UAM), or Ultrasonic Consolidation (UC) as it is also referred, is a hybrid form of additive and subtractive manufacturing technologies which is based on the sequential solid-state ultrasonic welding of metal foils. ${ }^{173}$ The process was developed in 2000 by Solidica Inc.. UAM combines ultrasonic welding with computer numerical control (CNC) machining, using an ultrasonic welder to sequentially join metallic foils together in a layer by layer process. This is achieved via a high frequency ultrasonic energy source generated from an ultrasonic transducer, which transfers energy to a body through a roughened sonotrode in the form of ultrasonic oscillation. These oscillations, combined with a compressive downward force from the sonotrode, allow inter-laminar metal flow with associated localised heating, resulting in metallurgical bonding at the consolidation interface. After each foil has been sequentially welded, the excess body of the part is removed via CNC milling. Repeating this process sequentially allows a $3 \mathrm{D}$ part to be manufactured. UAM is therefore a hybrid of additive and subtractive manufacturing techniques. This novel combination of techniques provides a number of unique manufacturing capabilities including bonding thermally and mechanically dissimilar materials into a single material component, and embedding functional components between foil layers. ${ }^{174}$ These capabilities are particularly pertinent for flow chemistry applications whereby embedded sensors could provide reaction feedback and analysis, and multi-material parts could have a range of applications including embedding catalytic sites or temperature controlled regions. 


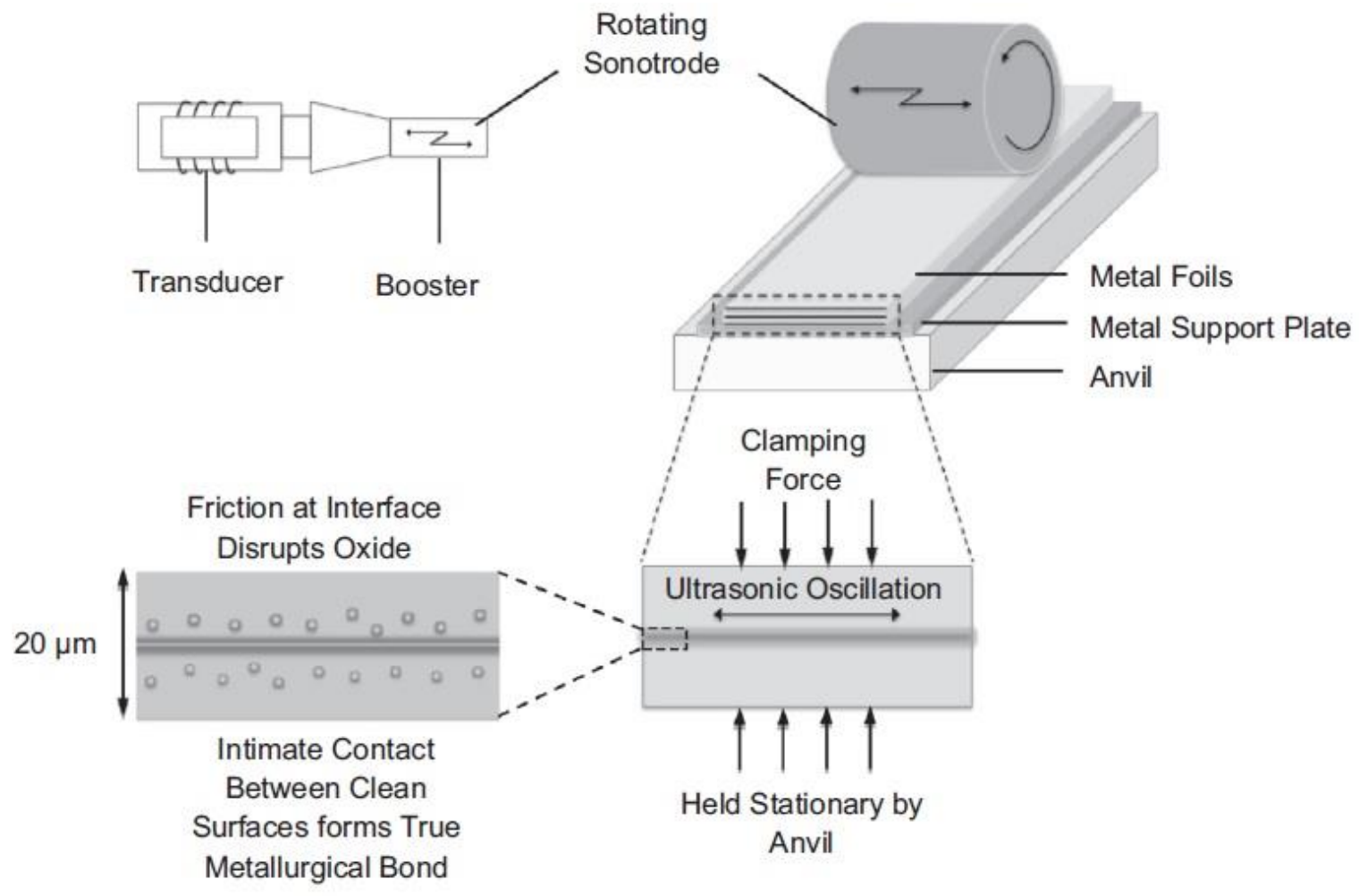

Figure 114: A Typical UAM Build Process. ${ }^{175}$

The machine used for this work was a Form-ation ${ }^{\mathrm{TM}}$ UAM machine (manufactured by Solidica, Inc. USA) with a Ti-6Al-4V sonotrode.

\subsection{Results and Discussion}

In Chapter 7.2 the difficulties of using temperature data generated from the inbuilt thermocouple of a heating mantle/block to monitor the temperature of a chemical reactor attached to it was discussed. The specific dimensions of the reactor, the thermal conductivity of the material, as well as the accuracy of the heating block, can all have a significant impact on the overall temperature of the reaction medium passing through the system. These temperature measurements will inevitably have a significant impact on the accuracy and repeatability of any automated reaction optimisation data generated. It was proposed that the chemical reactors could have a number of inbuilt thermocouples along the reaction pathway, so that temperature data generated would be specific not only to a single reactor but also a specific section of that reactor. For example, parts which have a number of temperature controlled regions would not be the same temperature as the heating mantle they are placed upon. It would also allow parts to be heated accurately on heating blocks which did not have accurate temperature measurement and control software. UAM has been shown to be an ideal 
manufacturing process for embedding discrete components such as thermocouples into an additively manufactured part.

\subsubsection{UAM Part 1}

\section{Design, build and post processing:}

Before embedding a thermocouple into a functioning reactor could be attempted, it was necessary to establish a set of weld parameters which would allow the thermocouple to be embedded, whilst maintaining the structural integrity of the part and the thermocouple. It was decided to try embedding a $200 \mu \mathrm{m}$ diameter type-k thermocouple $\left(-100\right.$ to $\left.250^{\circ} \mathrm{C}\right)$; this thermocouple was selected as it was small enough to embed between two foil layers, and would monitor temperatures across a wide range. The use of a $50 \mu \mathrm{m}$ thick $3003-\mathrm{H} 18$ grade of aluminium foil with a width of $25.4 \mathrm{~mm}$ was trialled as success welding these foils together had previously been had and therefore seemed a logical material to start with. ${ }^{175}$

Before embedding, the thermocouple must be stripped of its protective polymer coating. A 1 $\mathrm{mm}$ thick piece of 1050 aluminium (length $=300 \mathrm{~mm}$, width $=30 \mathrm{~mm}$ ) was used as the baseplate due to the fact it is a very soft alloy (Vickers Hardness 25). A single aluminium foil was welded to this base plate (weld force $=1600 \mathrm{~N}$, weld speed $20 \mathrm{~mm} / \mathrm{s}$, horn power 25 $\mathrm{W}$, weld distance $110 \mathrm{~mm}$ ). The relatively high weld force and horn power was selected to ensure complete consolidation of the foil to the base plate. A thermocouple was then secured in place using masking tape perpendicular to the weld direction, and a second foil was welded over the top (weld force $=1400 \mathrm{~N}$, weld speed $20 \mathrm{~mm} / \mathrm{s}$, horn power $20 \mathrm{~W}$, weld distance 110 $\mathrm{mm})$. The weld force and horn power were reduced to prevent cracking of the foil along the thermocouple/foil interface. Some difficulties were experienced with securing the thermocouple to the part during the weld process. Future research will look at methods of securing these thermocouples in place to prevent them moving during the weld process. 


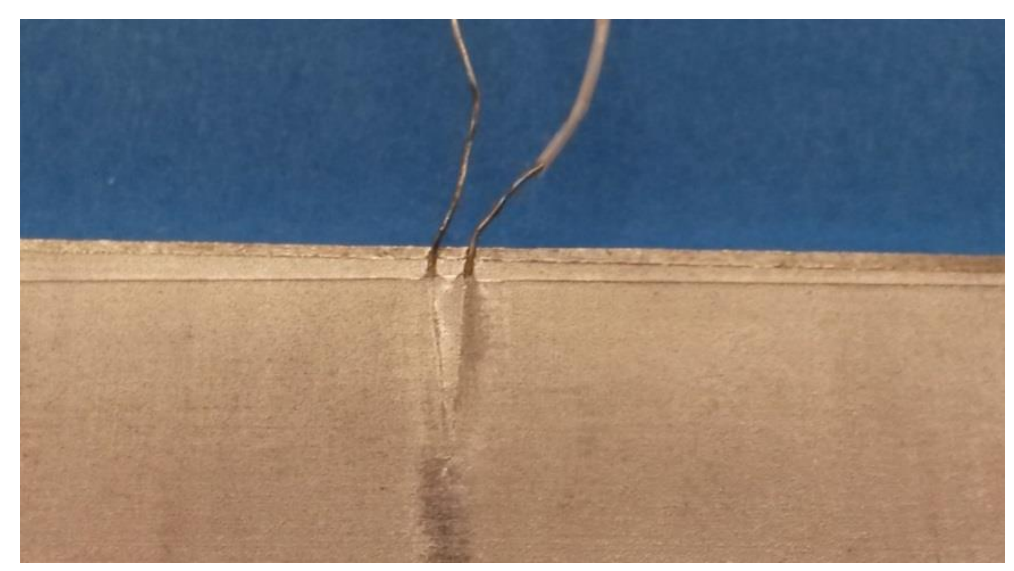

Figure 115: UAM Part 1.

\section{Results/Discussion:}

The thermocouple was connected to a PicoTech TC-08 Thermocouple Data Logger. The part was attached to the microchip heating block of a FlowSyn, with the thermocouple facing the block. The FlowSyn was programmed to heat from $25-100^{\circ} \mathrm{C}$ and the temperature displayed was recorded every 5 seconds. This was compared to the data produced from the thermocouples and recorded via the data logger (Figure 116).

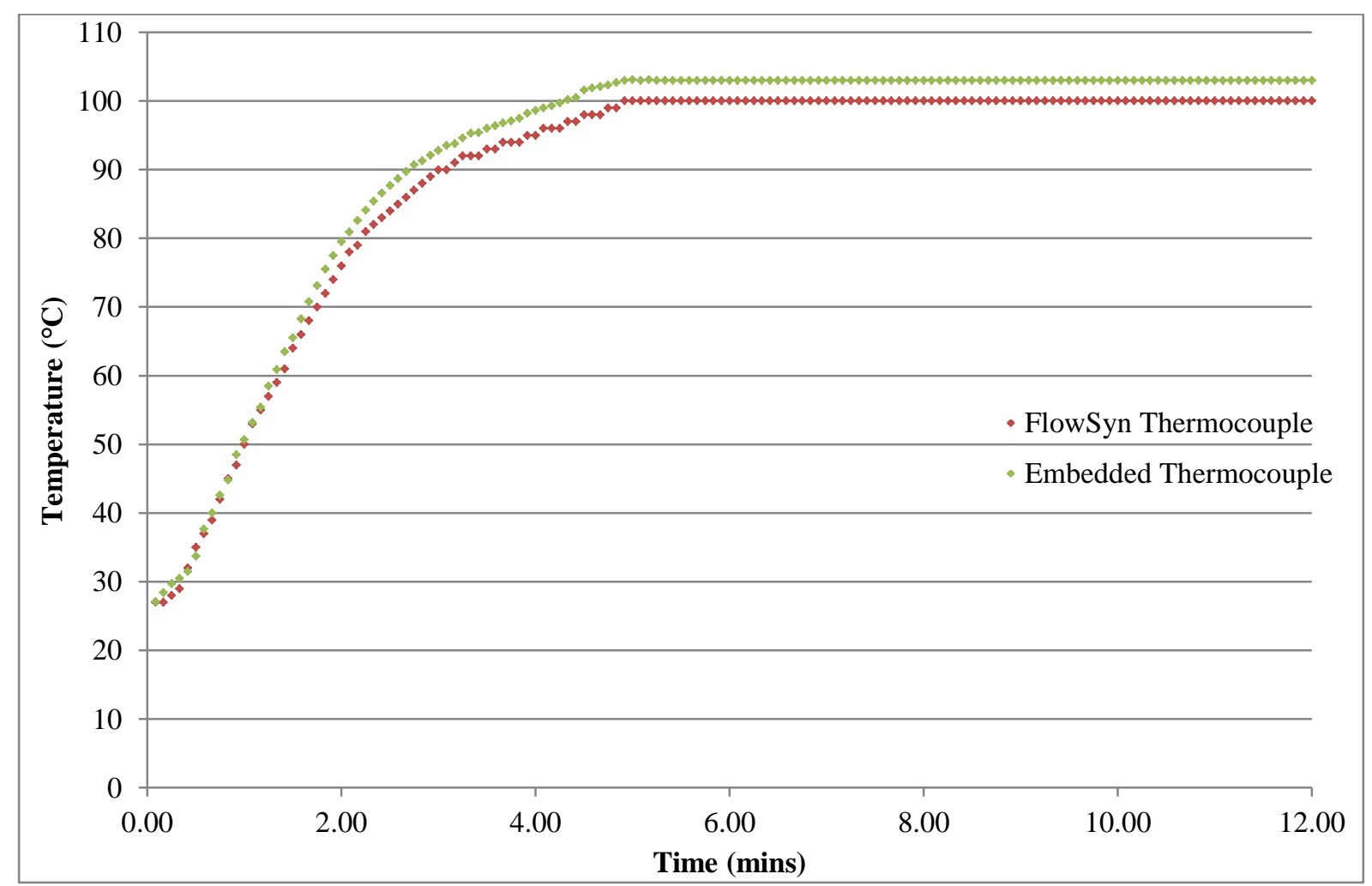

Figure 116: A Graph plotting the temperature profile of a thermocouple embedded between two foils heated by a Uniqsis FlowSyn, against the temperature readout from the FlowSyn thermocouple. 
The temperature reading from the embedded thermocouple was within $+/-5^{\circ} \mathrm{C}$ of the programmed temperature from the FlowSyn. This demonstrates that for precise measurements of optimum reaction conditions, embedded thermocouples may be a more accurate method of determining reaction temperature than using the standard machine readout. For the section of reactor in contact with the heated block, there was no delay in heat transfer throughout the part; a second part was manufactured in order to determine whether this effect would be true for a geometrically bulkier reactor.

\subsubsection{UAM Part 2}

\section{Design, build and post processing:}

For the manufacture of UAM part 2, a thicker $20 \mathrm{~mm} 1050$ aluminium base plate was selected $($ length $=300 \mathrm{~mm}$, width $=30 \mathrm{~mm}$ ). The same manufacturing process outlined in UAM part 1 was used to embed the thermocouple.

\section{Results/Discussion:}

Once again the part was attached to the FlowSyn which was programmed to heat from 25 $100^{\circ} \mathrm{C}$. This time the thermocouple was facing away from the heating block, so that there was $20 \mathrm{~mm}$ of aluminium separating the two readouts. 


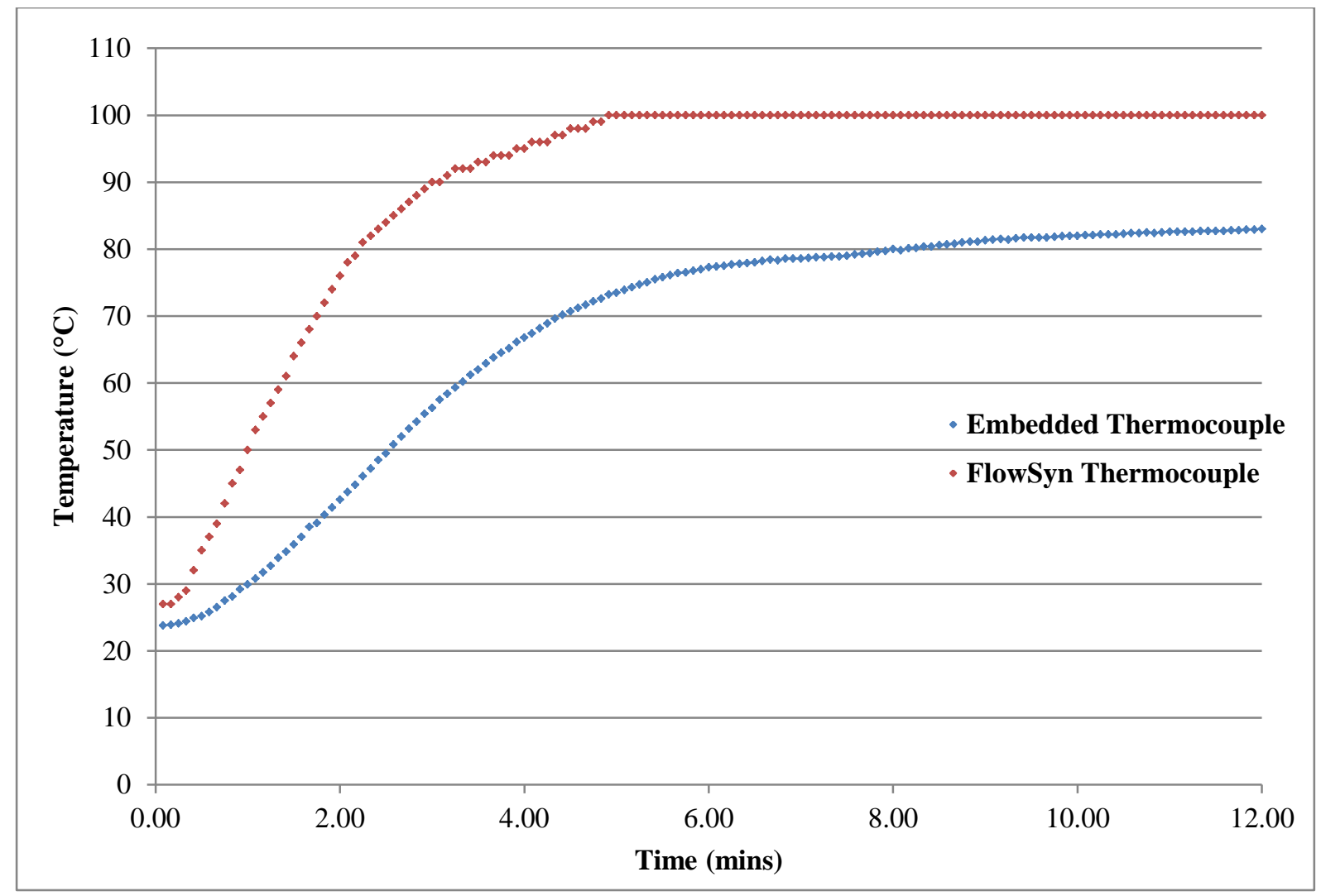

Figure 117: A Graph Plotting the Temperature Profile of a Thermocouple Embedded Between a $10 \mathrm{~mm}$ Aluminium Block and a 50 $\mu \mathrm{m}$ Foil Heated by a Uniqsis FlowSyn Against the Temperature Readout From the FlowSyn Thermocouple.

It was clear that as well as being a considerable delay in the heating profile of the embedded thermocouple, there was also a considerable discrepancy between the two values being generated $\left(\sim 20^{\circ} \mathrm{C}\right)$. This delay will vary with the thermal conductivity of the material, however needs to be considered when calculating the period of time any reaction requires to reach a steady state. Embedded thermocouples within the part will help overcome this issue by generating a true reading for the part. The discrepancy between the two values will depend on the environmental conditions within which the reaction is taking place (in particular the discrepancy between the efficiency of convective heating and radiative cooling of the material), and could lead to large variations in reaction conditions. However, by using the data generated from the thermocouples in a feedback control loop, it would be possible to control the energy outputted by any heating block, to ensure that the desired temperature of a reaction is reached. 


\subsubsection{UAM Part 3}

\section{Design, build and post processing:}

The next manufactured part was designed as a simple flow cell which would determine the temperature of the reaction medium as it passed through a reactor coil. Unfortunately, the Form-ation ${ }^{\mathrm{TM}}$ UAM used during this research can only manufacture parts which are $10 \mathrm{~mm}$ or less in thickness, due to the geometrical constraints of the machine. It was determined that a part of this thickness would be too thin to attach a threaded input and output port to. It was therefore necessary to manufacture UAM part 3 in two separate pieces, whereby the bottom piece would contain a flow channel with an embedded thermocouple, and the top piece would allow the part to be connected to the pumps. Again, due to the limitations of the equipment, it would be necessary to manually mill the appropriate flow paths and input/output ports of the reactor. Once the 2D flow path had been milled onto the surface of the part, the part would be capped with a lid by welding a single foil over the top of it, followed by a second foil which would contain the embedded thermocouple. The flow path that was designed was $30 \mathrm{~mm}$ in length, $2 \mathrm{~mm}$ in width and $1 \mathrm{~mm}$ in depth. The part was also designed so that it would attach to the microchip holder of the FlowSyn via two M4 screws.

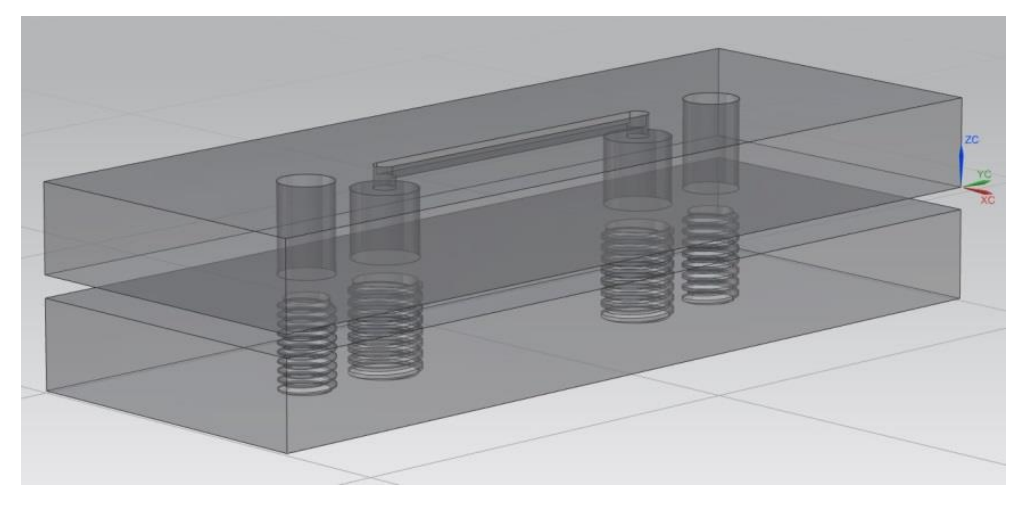

Figure 118: CAD Design for UAM Part 3.

The foils were welded to the part using the same processing parameters outlined in the design of UAM part 1. 


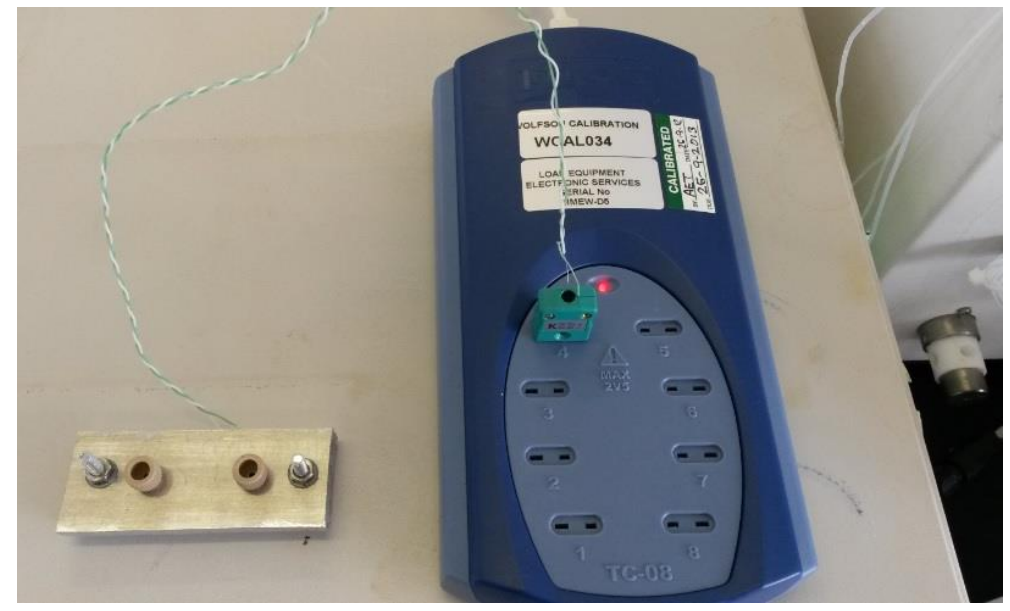

Figure 119: UAM Part 3 attached to a data logger.

\section{Results/Discussion:}

\section{Optimisation 6:}

Having produced a temperature controlled flow cell, it was decided that the data generated from the embedded thermocouple should be used as part of the optimisation feedback loop discussed during Chapters 6 and 7. This data could give a more accurate representation of the reaction temperature, and be used to control the reaction temperature by providing feedback to the heating block/mantle. Once again, the reaction of carvone outlined in Scheme 6 was chosen as the model reaction. In order to run the reaction, a binary pumping module was used to pump the two reagent flows which passed through a $5 \mathrm{~mL}$ stainless steel coil reactor. This reactor was attached to a heating mantle, and heated using the temperature controlled heating module of a Uniqsis FlowSyn. The flow would then pass into UAM part 3 which would be attached to the microchip heating block of the FlowSyn, which would be set to the same temperature as the reaction coil. The embedded thermocouple would be attached to a data logger which would generate the reaction temperature data. The flow would then pass into a 6 port valve, which would allow the flow to pass into either a collection vial, or be injected onto an Agilent 1100 series HPLC for data analysis. The analysis was set up with the conditions outlined in Table 74; 


\begin{tabular}{|c|c|c|c|}
\hline Reaction Volume & \multicolumn{3}{|c|}{$5.5 \mathrm{~mL}$} \\
\hline DAD settings & \multicolumn{3}{|c|}{$254,16 \mathrm{~nm}, 2 \mathrm{~nm}$ slit width } \\
\hline Column & Agilent Zorbax Eclipse Plus 4.6 x 50mmx3.5 um \\
\hline Column Temperature & \multicolumn{3}{|c|}{$30^{\circ} \mathrm{C}$} \\
\hline Mobile Phase & \multicolumn{3}{|c|}{ A: Water B:MeOH, 1 mL/min } \\
\hline \multirow{3}{*}{ Mobile Phase Gradient } & Time & A (\%) & 10 \\
\cline { 2 - 4 } & 0 & 90 & 10 \\
\cline { 2 - 4 } & 10 & 90 & 90 \\
\cline { 2 - 4 } & 16 & 10 & 10 \\
\cline { 2 - 4 } & 17 & 90 & 50 \\
\hline Reagent Inlet A & \multicolumn{3}{|c|}{0.31 mL carvone in 2000 mL MeOH } \\
\hline Reagent Inlet B & 0.46 g semicarbazide, 0.17 g NaOAc in 2000 mL water \\
\hline
\end{tabular}

Table 74: Reaction and analysis conditions used during optimisation 4.

Initially the control of the reaction conditions was carried out manually whilst the new optimisation system was tested. A wide range of reaction conditions were tested whilst the temperature data generated for each condition was stored for further analysis (Table 75). 


\begin{tabular}{|c|c|c|c|c|}
\hline $\begin{array}{c}\text { Reaction } \\
\text { Number }\end{array}$ & $\begin{array}{c}\text { Temperature } \\
\left({ }^{\circ} \mathrm{C}\right)\end{array}$ & $\begin{array}{c}\text { Flow Rate } \\
(\mathrm{mL} / \mathrm{min})\end{array}$ & $\begin{array}{c}\text { Residence } \\
\text { Time } \\
(\mathrm{mins})\end{array}$ & $\begin{array}{c}\text { Product } \\
\text { Conversion } \\
(\%)\end{array}$ \\
\hline 1 & 25 & 1 & 5.5 & 16.9 \\
\hline 2 & 25 & 0.5 & 11 & 22.4 \\
\hline 3 & 25 & 0.25 & 22 & 44.3 \\
\hline 4 & 25 & 0.1 & 55 & 49.8 \\
\hline 5 & 50 & 1 & 5.5 & 36.8 \\
\hline 6 & 50 & 0.5 & 11 & 40.7 \\
\hline 7 & 50 & 0.25 & 22 & 57.4 \\
\hline 8 & 50 & 0.1 & 55 & 52.4 \\
\hline 9 & 75 & 1 & 5.5 & 51.5 \\
\hline 10 & 75 & 0.5 & 11 & 52.2 \\
\hline 11 & 75 & 0.25 & 22 & 58.3 \\
\hline 12 & 75 & 0.1 & 55 & 69.0 \\
\hline
\end{tabular}

Table 75: Reaction Conditions Used During Optimisation 6.

This data is displayed in Figure 120.

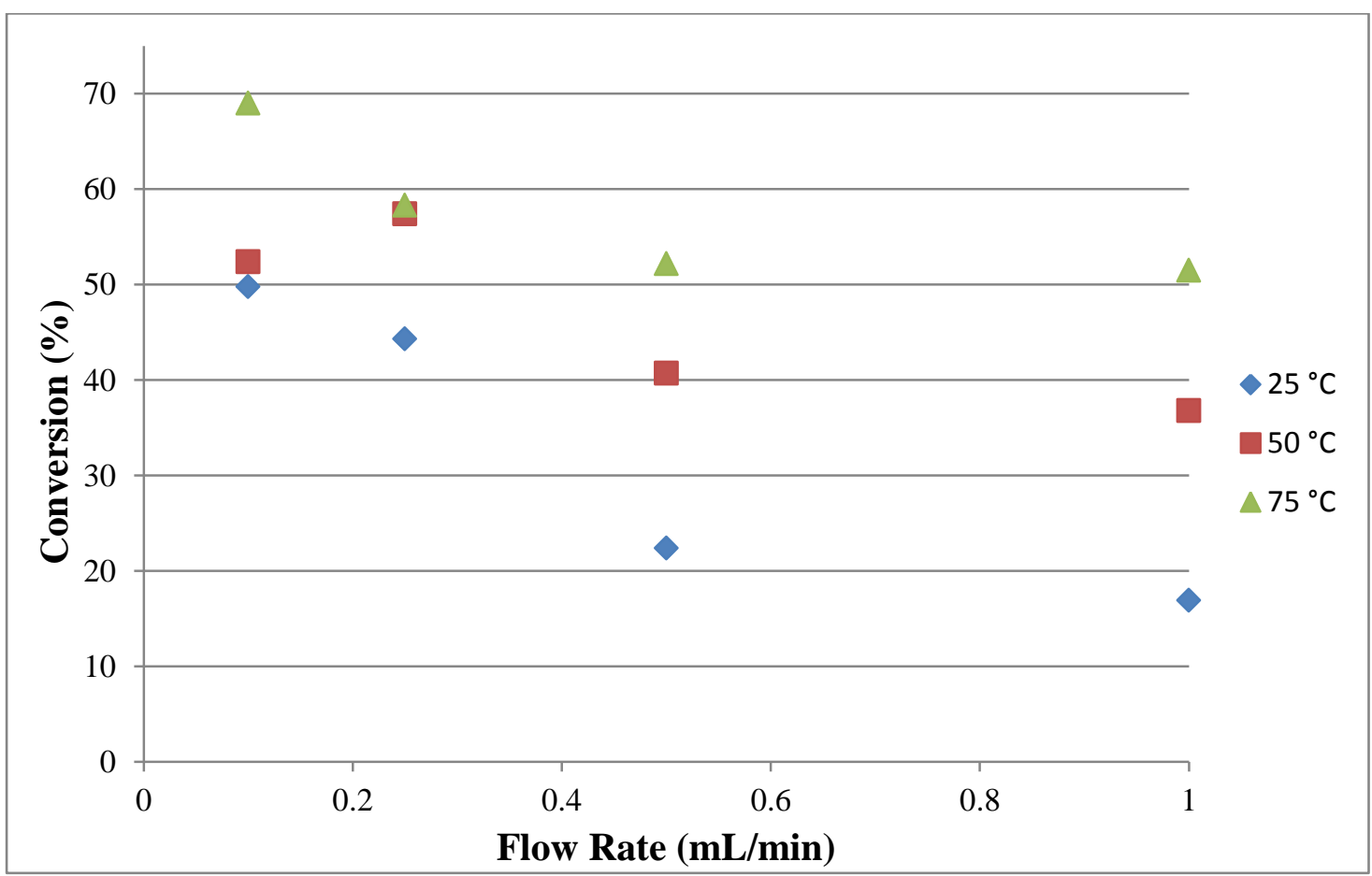

Figure 120: Graph Showing the Data Generated from Optimisation 6. 
The data logger was setup to record the temperature of the reaction every 30 seconds. Figure 121 depicts the temperature data recorded during the three reactions run at $1 \mathrm{~mL} / \mathrm{min}$, comparing it to the pre-programmed temperature of the FlowSyn. The data was recorded for the first 5 minutes of each set of reaction conditions.

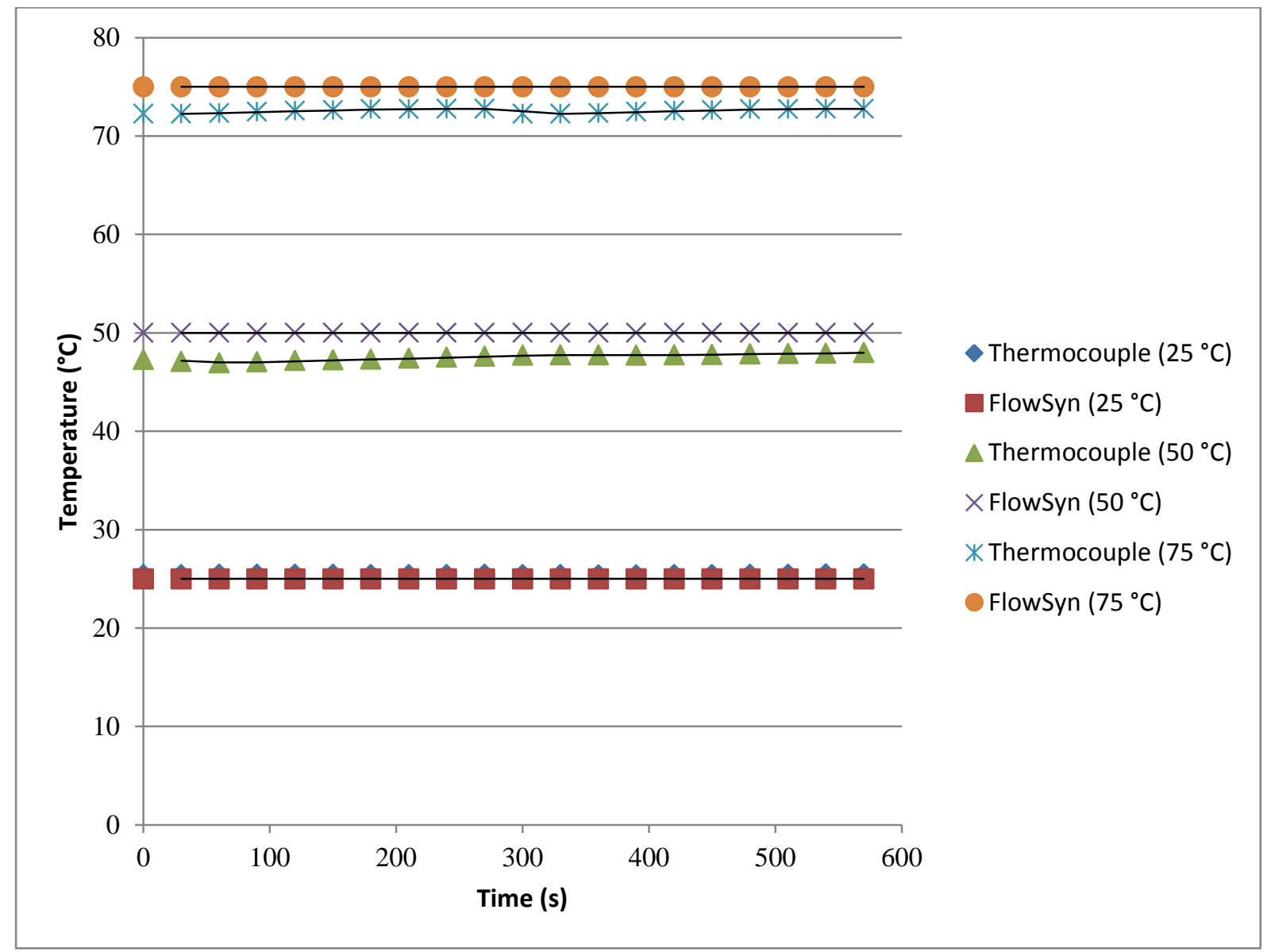

Figure 121: Thermocouple Data Generated During Optimisation 6 for the Reactions Run at $1 \mathrm{~mL} / \mathrm{min}$.

It is clear that there is a discrepancy $\left(\sim 2^{\circ} \mathrm{C}\right)$ between the programmed temperature from the FlowSyn and the actual temperature being generated within the reactor. Although this discrepancy is largely consistent and would not affect optimisation trends substantially, it still represents and error within the system which could lead to incorrect conclusions about optimum reaction conditions which would not be repeatable between different reaction setups. Work in this area is ongoing, with the aim of producing a range of functional analytical flow devices via UAM. 


\subsection{Conclusion}

The UAM process offers considerable scope in terms of embedding functionality into flow devices in the form of a range of sensors and detectors, which could be used as part of a wider automated flow system. Unfortunately, UAM is still in early development stages as an AM process, and still requires considerable research and development to optimise. In this research, this is characterised by the inability to produce complex $3 \mathrm{D}$ structures without painstaking effort to individually mill each layer of the part. However, it may be possible to hybridise UAM with other existing metal AM processes such as SLM. This would allow the user to incorporate the complex designs that can be produced via SLM, whilst adding functionality to those parts via embedded UAM components.

\subsection{Future Work}

Future work will focus on developing a series of macros which can process the large amount of data generated from the embedded thermocouples. These macros will need to process the data and output it as a single value (probably an average reaction temperature), allowing the data to be inputted into the optimisation software. It would also be of interest to manufacture parts with multiple embedded thermocouples, allowing the part to have a number of temperature controlled regions which could be individually monitored. The data generated could also be used prior to the reaction conditions being run, to ensure that the reactor temperature is exactly the same as the pre-programmed temperature. As well as this, ongoing research will focus on using UAM to embed optical fibres within the flow path of a reactor, allowing the generation of inline spectroscopic data from a number of points along the flow path. This data could once again be used as a feedback mechanism for the reaction optimisation setup. Other areas of interest will be the production of multi-material parts, as well as embedding a range of other components such as flow rate sensors and switching valves. 


\section{Summary and Concluding Remarks}

\begin{tabular}{|c|c|c|c|c|}
\hline$\underset{\text { Process }}{\text { AM }}$ & $\begin{array}{l}\text { Currently } \\
\text { Available } \\
\text { Materials }\end{array}$ & $\begin{array}{l}\text { Chemical } \\
\text { Stability }\end{array}$ & Thermal Stability & $\begin{array}{l}\text { Achievable } \\
\text { Build } \\
\text { Resolutions }\end{array}$ \\
\hline FDM & $\begin{array}{l}\text { ABS, PLA, PA, } \\
\text { PC, ULTEM®. }\end{array}$ & $\begin{array}{l}\text { FDM materials } \\
\text { range in chemical } \\
\text { stability, however } \\
\text { typically the ester } \\
\text { linkages of } \\
\text { polymers like PC } \\
\text { and ULTEM® are } \\
\text { susceptible to } \\
\text { chemical } \\
\text { degradation. }\end{array}$ & $\begin{array}{c}\text { FDM materials range } \\
\text { in thermal stability. } \\
\text { ABS has a high glass } \\
\text { transition } \\
\text { temperature of } \\
220^{\circ} \mathrm{C} \text {, whereas } \mathrm{PC} \\
\text { has a lower glass } \\
\text { transition } \\
\text { temperature of } 147 \\
{ }^{\circ} \mathrm{C} \text {. }\end{array}$ & $\begin{array}{c}\text { Generally } \\
\text { acceptable, with } \\
\text { build resolutions } \\
\text { of }+/-0.1 \mathrm{~mm} \\
\text { possible. Needs } \\
\text { support } \\
\text { structures for } \\
\text { smaller } \\
\text { geometries. }\end{array}$ \\
\hline LS & $\begin{array}{c}\text { Typically } \\
\text { polymers such as } \\
\text { Nylon-11 and }-12 \text {, } \\
\text { PP, PEEK and } \\
\text { some } \\
\text { experimental } \\
\text { ceramics. }\end{array}$ & $\begin{array}{l}\text { Amide linkages of } \\
\text { polymers such as } \\
\text { Nylon- } 12 \text { are } \\
\text { susceptible to } \\
\text { strong bases and } \\
\text { nucleophiles. } \\
\text { PEEK is a } \\
\text { material widely } \\
\text { used in flow } \\
\text { chemistry. }\end{array}$ & $\begin{array}{l}\text { Wide ranging but } \\
\text { generally stable at } \\
\text { high temperatures. } \\
\text { The most frequently } \\
\text { used Nylon- } 12 \text { has a } \\
\text { heat deflection } \\
\text { temperature of } 177 \\
{ }^{\circ} \mathrm{C} \text { at } 66 \text { psi. }\end{array}$ & $\begin{array}{l}\text { Comparable or } \\
\text { even slightly } \\
\text { better than FDM, } \\
\text { with build } \\
\text { resolutions of +/- } \\
0.15 \mathrm{~mm} \\
\text { possible. }\end{array}$ \\
\hline SL & $\begin{array}{l}\text { UV curable } \\
\text { photopolymers, } \\
\text { typically acrylate } \\
\text { or epoxy } \\
\text { backboned. }\end{array}$ & $\begin{array}{l}\text { Poor chemical } \\
\text { stability, } \\
\text { significant } \\
\text { degradation and } \\
\text { swelling in } \\
\text { stronger solvent } \\
\text { systems due to } \\
\text { weak epoxy and } \\
\text { acrylate } \\
\text { backbones. }\end{array}$ & $\begin{array}{c}\text { Huge range of SL } \\
\text { materials available, } \\
\text { however generally } \\
\text { very poor. Accura } 60 \\
\text { has a low glass } \\
\text { transition } \\
\text { temperature of } 58^{\circ} \mathrm{C} \text {. } \\
\text { Accura Bluestone } \\
\text { marketed as a high } \\
\text { temperature material } \\
\text { has a glass transition } \\
\text { temperature of } 83^{\circ} \mathrm{C} .\end{array}$ & $\begin{array}{l}\text { Excellent, with } \\
\text { build resolutions } \\
\text { of less than +/- } \\
0.05 \mathrm{~mm} \\
\text { possible. }\end{array}$ \\
\hline SLM & $\begin{array}{l}\text { Steel, titanium, } \\
\text { aluminium, etc. }\end{array}$ & $\begin{array}{l}\text { Highly stable } \\
\text { metal oxide layers } \\
\text { make SLM parts } \\
\text { chemical resistant } \\
\text { to a wide range of } \\
\text { reagents. }\end{array}$ & $\begin{array}{l}\text { Thermally stable to } \\
\text { extremely high } \\
\text { temperatures. } \\
\text { Aluminium is stable } \\
\text { up to its melting } \\
\text { point of } 660^{\circ} \mathrm{C} \text { and } \\
\text { titanium has a } \\
\text { melting point of } \\
\text { around } 1660^{\circ} \mathrm{C} \text {. }\end{array}$ & $\begin{array}{c}\text { Excellent, with } \\
\text { build resolutions } \\
\text { of less than }+/- \\
0.05 \mathrm{~mm} \\
\text { possible. } \\
\text { However, often } \\
\text { requires more } \\
\text { build process } \\
\text { optimisation than } \\
\text { other techniques. }\end{array}$ \\
\hline UAM & $\begin{array}{l}\text { Aluminium, } \\
\text { copper etc. }\end{array}$ & $\begin{array}{c}\text { Highly stable } \\
\text { metal oxide layers } \\
\text { make UAM parts } \\
\text { chemical resistant } \\
\text { to a wide range of } \\
\text { reagents. }\end{array}$ & $\begin{array}{l}\text { Thermally stable to } \\
\text { extremely high } \\
\text { temperatures. } \\
\text { Aluminium is stable } \\
\text { up to its melting } \\
\text { point of } 660^{\circ} \mathrm{C} \text {. }\end{array}$ & $\begin{array}{c}\text { Relies on } \\
\text { subtractive } \\
\text { milling processes } \\
\text { - theoretically } \\
\text { capable of very } \\
\text { high resolution } \\
\text { builds. }\end{array}$ \\
\hline
\end{tabular}

Table 76: A comparison of the materials properties, design requirements and engineering considerations each AM manufacturing technique reviewed above. 
The additive manufacturing techniques presented each demonstrate significant future scope in terms of chemical reactor design and manufacture. However, each of the mentioned techniques is not without its own engineering and material issues, which need to be fully considered before they could be used to manufacture chemical reactors. The applicability of each technique is assessed in the table below. For each technique, a number of distinct materials and equipment types are available, making thorough comparisons of each technique complex, but guideline information for each process has been included in Table 76.

FDM is perhaps the most widely used AM process for the manufacture of microfluidic devices. The FDM process is ideally suited to manufacturing simplistic microfluidic geometries, due to the fact that the parts can be produced in a cost and time efficient manner. This allows the parts to be treated as disposable, often overcoming some of the issues with this process associated with the physical and chemical properties of the parts produced. Due to the user friendly consumer nature of this process, there is also significant scope for research teams around the globe to share designs and build parameters, allowing reaction setups to be replicated in any laboratory. However, there are still significant issues to be overcome before this process can be considered a serious alternative to existing manufacturing processes. Firstly, despite being continually developed, the list of printable materials is still a stumbling block for most fluidic processes. As well as this there are still some issues with build resolution, reproducibility and more fundamentally the ability to produce a completely dense and water-tight part that can withstand elevated temperatures and pressures.

LS is a manufacturing process that offers a significant amount towards microfluidic device manufacture in terms of material properties as well as achievable part resolution. However, it would take some significant research to establish the correct processing conditions to facilitate powder removal from micron sized internal features. If these conditions were found or if the current trend of improving commercially available equipment continues, then this process may have a significant contribution to the field of microfluidic manufacture. For the time being though it perhaps remains too costly a process to pursue, given the potential offered by some of the other additive manufacturing toolbox already available.

SL has proven to be an excellent process for the manufacture of high precision microfluidic pieces. It can produce high resolution, fully dense parts that do not require much post processing. Although this process is more expensive than FDM, it is still considerably 
cheaper than other conventional manufacturing processes and therefore has a significant future in the field. The main limitation of the process is the availability of materials that possess the appropriate chemical properties for fluidic applications. Many of the materials are not solvent friendly, and experience distortion over prolonged use. As well as this many of the materials are thermally insulating and are therefore not ideally suited to heated fluidic applications. One area where there is significant promise for this process is the manufacture of bio-reactors. In recent years a number of commercially available bio-resins have been given FDA approval, and are therefore suitable for use with specific cell lines. This materials portfolio is ever expanding and will enable a full spectrum of bio applications to be feasible using this process.

SLM is another process that has huge potential for the manufacture of fluidic devices. The parts produced are chemically compatible across a wide range of applications, thermally conductive, capable of withstanding significant pressures and are fully dense. It has been shown that with the right processing conditions powder removal is possible at very small geometries and the build resolution of commercially available equipment is improving at a rapid rate. The only issue that the process potentially has is that the cost of operating SLM equipment, or even purchasing the parts from a supplier is still quite high. Despite this SLM is now a viable alternative to conventional manufacturing process for milli-scale fluidics, and in the coming years will also become more common place for the manufacture of micro-scale devices as well.

Finally, UAM is perhaps the process furthest away from widespread use for the manufacture of microfluidics, but perhaps with the largest upside. At this point in time the process is still very much a developmental one and perhaps only suited to research laboratories. It does however offer huge potential in the areas of embedded technology which would allow the manufacture of devices with a significant amount of added functionality to the user.

It is clear from these studies that despite some clear limitations, AM offers huge potential in terms of chemical reactor design and manufacture. The user involvement with the entire design and manufacturing process allows precise control and rapid optimisation of reactor dimensions, geometry and functionality. The integration of sensors and detectors into parts for inline reaction analysis offers huge potential gains over existing flow devices. This level of design freedom is not currently available when using existing microreactor fabrication methods. Additional benefits of AM procedures, such as an increased speed of production 
and reduced manufacturing costs, show that AM processes have significant scope to improve on the existing portfolio of microreactor fabrication processes.

This research has demonstrated a range of complex $3 \mathrm{D}$ geometries that can be applied to flow chemistry applications. It has also identified several current areas of weakness that the industry leading specification machines used in these studies still encounter. However, AM is a quickly evolving field, with new technologies, systems and the types of materials that can be processed continually being developed, which should help rapidly advance the portfolio of reactor designs possible using these manufacturing methods. 


\section{Experimental}

\subsection{Instrumentation and Software}

\subsubsection{Infrared Spectroscopy}

All infrared spectra were obtained using a Perkin-Elmer 65 FT-IR spectrophotometer. Spectra for liquids were acquired as a thin film using sodium chloride plates or in the case of solids, as a $\mathrm{NaCl}$ disc.

\subsubsection{NMR}

All ${ }^{1} \mathrm{H}$ and ${ }^{13} \mathrm{C}$ NMR spectra were measured at 400 and $100 \mathrm{MHz}$ respectively using a Bruker DPX $400 \mathrm{MHz}$ spectrometer. The solvent used for NMR spectroscopy was chloroform- $d_{1}(\delta$ 7.26, ${ }^{1} \mathrm{H} ; \delta 77.0,{ }^{13} \mathrm{C}$ ) using TMS (tetramethylsilane) as the internal reference, unless stated otherwise. Chemical shifts are given in parts per million (ppm) and $J$ values are given in Hertz $(\mathrm{Hz})$.

\subsubsection{Column Chromatography}

All chromatographic manipulations used silica gel as the adsorbent. Reactions were monitored using thin layer chromatography (TLC) on aluminium backed plates with Merck TLC $60 \mathrm{~F}_{254}$ silica gel. TLC visualised by UV radiation at a wavelength of $254 \mathrm{~nm}$, or stained by exposure to an ethanolic solution of vanillin (acidified with concentrated sulfuric acid) or an ethanolic solution of Phosphomolybdic acid, followed by charring where appropriate. Purification by column chromatography using Apollo ZEOprep 60/ 40-63 $\mu \mathrm{m}$ silica gel.

\subsubsection{Pumping Equipment}

Flow was achieved within the AM devices via a number of different pumping technologies including a FlowSyn continuous flow system, an Agilent 1100 series binary pumping module, a Thompson single step pump, and a Semat A-99 battery powered syringe pump. 


\subsubsection{High Performance Liquid Chromatography}

All HPLC analysis was conducted using an Agilent 1100 series LC system. This system consisted of two binary pumping modules, an autosampler, heated column compartment, and DAD. Details of individual LC runs are given in the results and discussion.

\subsubsection{Imaging}

$\mu \mathrm{CT}$ imaging of SLM Part 7 was carried out using an Xradia VersaXRM 500 3D X-Ray Microscope with the following parameters.

\begin{tabular}{|c|c|c|c|}
\hline Imaging Mode & Setting & Source Information & Setting \\
\hline Camera Temperature & $-59\left({ }^{\circ} \mathrm{C}\right)$ & Power & $9.97 \mathrm{~W}$ \\
\hline Source Setting & $160 \mathrm{kV}, 63 \mu \mathrm{A}$ & Voltage & $159.85 \mathrm{~V}$ \\
\hline Source Distance & $200.1037 \mathrm{~mm}$ & Target Current & $62.36 \mathrm{~A}$ \\
\hline Detector Distance & $35.6731 \mathrm{~mm}$ & Tube Current & $220.44 \mathrm{~A}$ \\
\hline Pixel Size & $57.2995 \mu \mathrm{m}$ & Hours on Target & $15.32 \mathrm{~h}$ \\
\hline Optical Magnification & 0.39991 & Centering X & $-137.62 \mathrm{~mm}$ \\
\hline Exposure Time & $18 \mathrm{~s}$ & Centering X & $93.74 \mathrm{~mm}$ \\
\hline Camera Readout & $2.5 \mathrm{MHz}$ & & \\
\hline Image Size & $1024 \times 1024$ & & \\
\hline
\end{tabular}

Table 77: Scan settings for $\mu \mathrm{CT}$ imaging of SLM Part 7.

Optical microscopy was carried out using a GX Microscope L2003B HTG capable of x5, x10, x20, x40, and x60 magnification.

Scanning electron microscopy images were taken using a LEO 440. Reconstruction of the SEM images were conducted using Image J $1.48 \mathrm{~V}$, with the BoneJ plugin installed.

Non-contact scanning topography was undertaken using a Talysurf CLI 2000 Machine 3D surface profiler. 


\subsubsection{FDM Equipment}

Two FDM machines were used during the course of the research. A PP3DP UP! Printer was used to manufacture ABS parts. ABS reel was purchased from Maplin and was used as supplied. Parts were manufactured using the following build parameters;

\begin{tabular}{|c|c|}
\hline Setting & Value \\
\hline Extrusion Temperature & $260^{\circ} \mathrm{C}$ \\
\hline Extrusion Speed & $50 \mathrm{~mm} / \mathrm{s}$ \\
\hline Heated Bed Temperature & $100^{\circ} \mathrm{C}$ \\
\hline Z Resolution & $0.2 \mathrm{~mm}$ \\
\hline Base Height & $2 \mathrm{~mm}$ \\
\hline Fill & Dense \\
\hline Part Angle & $45^{\circ}$ \\
\hline Part Surface & 3 layers \\
\hline Support Density & 3 layers \\
\hline Support Space & 8 lines \\
\hline Support Area & 4 mm ${ }^{2}$ \\
\hline Support Angle & $30^{\circ}$ \\
\hline Build Quality & Fine \\
\hline
\end{tabular}

Table 78: Build parameters used when manufacturing ABS parts using an UP! Printer.

A MakerBot Replicator was used to manufacture PLA parts. PLA reel was purchased from MakerBot and was used as supplied. Parts were manufactured using the following build parameters;

\begin{tabular}{|c|c|}
\hline Setting & Value \\
\hline Extrusion Temperature & $210^{\circ} \mathrm{C}$ \\
\hline Extrusion Speed & $100 \mathrm{~mm} / \mathrm{s}$ \\
\hline Heated Bed Temperature & $50^{\circ} \mathrm{C}$ \\
\hline
\end{tabular}

Table 79: Build parameters used when manufacturing PLA parts using a MakerBot Replicator 2. 


\subsubsection{LS Equipment}

LS parts were produced using an EOS FORMIGA P 100 LS machine. All parts were manufactured from DuraForm ${ }^{\circledR}$ PA (Nylon). PA 2200 was purchased from 3D Systems and was used as supplied. Parts were manufactured using the build parameters outlined in table 79 ;

\begin{tabular}{|c|c|}
\hline Setting & Value \\
\hline Left and right feed heater set point & $130^{\circ} \mathrm{C}$ \\
\hline Part heater set point & $142^{\circ} \mathrm{C}$ \\
\hline Powder layer thickness & $0.1 \mathrm{~mm}$ \\
\hline Left and right feed distance & $0.254 \mathrm{~mm}$ \\
\hline Fill laser power & $11 \mathrm{~W}$ \\
\hline Fill beam offset (X and Y) & $0.254 \mathrm{~mm}$ \\
\hline Outline laser power & $5 \mathrm{~W}$ \\
\hline Outline scan count & 1 \\
\hline Outline beam offset (X and Y) & $0.229 \mathrm{~mm}$ \\
\hline Slicer fill scan spacing & $0.15 \mathrm{~mm}$ \\
\hline
\end{tabular}

Table 80: Build parameters used when manufacturing PA 2200 parts using a EOS FORMIGA P 100.

\subsubsection{UAM Equipment}

UAM parts were manufactured using a Form-ation ${ }^{\mathrm{TM}} \mathrm{UC}$ machine (manufactured by Solidica, Inc. USA) with Ti-6Al-4V sonotrode. The foils used were $50 \mu \mathrm{m}$ thick $3003-\mathrm{H} 18$ aluminium alloy with a width of $25 \mathrm{~mm}$.

\subsubsection{SLM Equipment}

SLM parts were produced using a Renishaw AM250, Realizer SLM 100 or a Realizer SLM 50. Parts were manufactured from 316L stainless steel and Ti-6Al-4V titanium. Parts were manufactured according to manufacturers recommended processing conditions, such as the following build parameters when using the Renishaw AM250 to manufacture parts from Ti$6 \mathrm{Al}-4 \mathrm{~V}$ 


\begin{tabular}{|c|c|}
\hline Outer Shell Build Parameters & Value \\
\hline Build Style & Contour \\
\hline Contours & 1330 \\
\hline Hatches & 167383 \\
\hline Volume Border Build Parameters & Value \\
\hline Distance & $52.6 \mathrm{~m}$ \\
\hline Exposure Time & $14.6 \mathrm{~h}$ \\
\hline Speed & $1 \mathrm{~mm} / \mathrm{s}$ \\
\hline Power Output & $140 \mathrm{~W}$ \\
\hline Focus Offset & $11 \mathrm{~mm}$ \\
\hline Point Distance & $20 \mu \mathrm{m}$ \\
\hline Point Exposure Time & $65 \mu \mathrm{s}$ \\
\hline Volume Area Build Parameters & Value \\
\hline Distance & $1238.6 \mathrm{~m}$ \\
\hline Exposure Time & $344.1 \mathrm{~h}$ \\
\hline Speed & $1 \mathrm{~mm} / \mathrm{s}$ \\
\hline Power Output & $200 \mathrm{~W}$ \\
\hline Focus Offset & $-12.5 \mathrm{~mm}$ \\
\hline Point Distance & $45 \mu \mathrm{m}$ \\
\hline Point Exposure Time & $190 \mu \mathrm{s}$ \\
\hline Volume Area Build Parameters & Value \\
\hline Distance & $51.9 \mathrm{~m}$ \\
\hline Exposure Time & $14.4 \mathrm{~h}$ \\
\hline Speed & $1 \mathrm{~mm} / \mathrm{s}$ \\
\hline Power Output & $200 \mathrm{~W}$ \\
\hline Focus Offset & $11 \mathrm{~mm}$ \\
\hline Point Distance & $65 \mu \mathrm{m}$ \\
\hline Point Exposure Time & $225 \mu \mathrm{s}$ \\
\hline
\end{tabular}

Table 81: Build parameters used when manufacturing SLM parts using a Renishaw AM250. 


\subsubsection{SL Equipment}

SL parts were produced using a 3D Systems Viper si2 SL system. Parts were manufactured from Accura 60 resin, which was supplied by 3D systems and used from a sealed container. The processing parameters were as outlined in table 81;

\begin{tabular}{|c|c|}
\hline Setting & Value \\
\hline Laser Power & $18 \mathrm{~mW}$ \\
\hline Border speed & 10.4 units/s \\
\hline Hatch speed & 50.7 units/s \\
\hline Fill speed & 27.1 units/s \\
\hline Material & Accura 60 \\
\hline Dip Distance & 0 \\
\hline Pre Drip & 0 \\
\hline Z Wait & 1 \\
\hline Sweeps & 1.9685 units/s \\
\hline Sweep Velocity & 0.0120 in \\
\hline Preferred Blade Gap & $0.1 \mathrm{~mm}$ \\
\hline Layer Thickness & 400 \\
\hline Blade gap \% & 50 units/s \\
\hline Blade Velocity & 0 \\
\hline
\end{tabular}

Table 82: Build parameters used when manufacturing Accura 60 parts using a Viper si2 SL system.

\subsubsection{CAD and .STL Conversion}

CAD drawings were produced using the commercially available software NX 7.5 and 8.5 (Siemens Industry Software), and converted to .STL file format for visualisation in MiniMagics 2.0 (Materialise).

\subsubsection{Reaction Optimisation}

Reaction optimisation software was written in MacroPad version 6.7.1.110, Microsoft Excel 2010 Visual Basics and Chemstation. 


\subsection{Laboratory Procedures}

\section{General procedure for the oxidation of aldehydes to methyl esters}

An aldehyde (4 mmol) and tetra-butyl ammonium bromide (132 $\mathrm{mg}, 0.4 \mathrm{mmol})$ were dissolved in methanol $(1.65 \mathrm{~mL}, 40 \mathrm{mmol})$ and made up to $5 \mathrm{~mL}$ with ethyl acetate. The solution was transferred to a small glass sample vial. To a second glass vial sodium hypochlorite $(5 \mathrm{~mL}, 12.5 \%$ solution in water) was added. The two solutions were flowed through the reactor at $0.38 \mathrm{mLmin}^{-1}$ giving a $t_{R}$ of 30 minutes. Reactant products were collected and extracted using ethyl acetate $(3 \times 25 \mathrm{~mL})$ and distilled water $(25 \mathrm{~mL})$. The combined organic layers were dried over anhydrous magnesium sulfate and evaporated to dryness under vacuum. Where appropriate the crude oil was purified using column chromatography to yield the pure product. Samples were analysed using ${ }^{1} \mathrm{H}$ NMR and IR spectroscopy.<smiles>COC(=O)c1ccccc1</smiles>

Methyl Benzoate (5.2.2). Clear colourless liquid, (93\%): ${ }^{1} \mathrm{H}$ NMR (400 MHz; $\left.\mathrm{CDCl}_{3}\right) \delta 3.92$ (s, 3H, $\mathrm{CH}_{3}$ ), 7.42-7.64 (m, 3H, Ar-H), 8.05 (m, 2H, Ar-H). IR (NaCl) v 3028, 2671, 1710, $1494,1416,1289,1223,970,699,489 \mathrm{~cm}^{-1}$.<smiles>COC(=O)c1ccc(C)cc1</smiles>

Methyl p-Toluate Colourless solid, (54\%). ${ }^{1} \mathrm{H}$ NMR (400 MHz; $\left.\mathrm{CDCl}_{3}\right) \delta 2.39\left(\mathrm{~s}, 3 \mathrm{H}, \mathrm{CH}_{3}\right)$, 3.89(s, 3H, $\left.\mathrm{CH}_{3}\right) 7.21-7.23(\mathrm{~d}, J=8.0 \mathrm{~Hz}, 2 \mathrm{H}, \mathrm{Ar}-\mathrm{H}), 7.92(\mathrm{~d}, J=8.0 \mathrm{~Hz}, 2 \mathrm{H}, \mathrm{Ar}-\mathrm{H})$. 
<smiles>COC(=O)c1ccc([N+](=O)[O-])cc1</smiles>

Methyl p-Nitrobenzoate Yellow solid, (100\%). ${ }^{1} \mathrm{H}$ NMR (400 MHz; $\left.\mathrm{CDCl}_{3}\right) \delta 3.99(\mathrm{~s}, 3 \mathrm{H}$, $\left.\mathrm{CH}_{3}\right) 7.22(\mathrm{~d}, J=11.2 \mathrm{~Hz}, 2 \mathrm{H}, \mathrm{Ar}-\mathrm{H}), 7.92(\mathrm{~d}, J=10.8 \mathrm{~Hz}, 2 \mathrm{H}, \mathrm{Ar}-\mathrm{H})$.

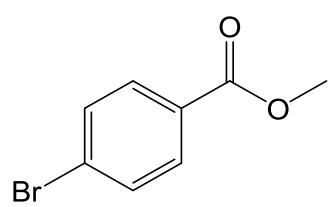

Methyl p-Bromobenzoate White solid, (100\%). ${ }^{1} \mathrm{H}$ NMR (400 MHz; $\left.\mathrm{CDCl}_{3}\right) \delta 3.92(\mathrm{~s}, 3 \mathrm{H}$, $\left.\mathrm{CH}_{3}\right)$ 7.57-7.59 (m, 2H, Ar-H), 7.89-7.91 (m, 2H, Ar-H).<smiles>COC(=O)c1cccc([N+](=O)[O-])c1</smiles>

Methyl m-Nitrobenzoate Yellow solid, (99\%). ${ }^{1} \mathrm{H}$ NMR (400 MHz; $\left.\mathrm{CDCl}_{3}\right) \delta 4.00(\mathrm{~s}, 3 \mathrm{H}$, $\mathrm{CH}_{3}$ ), 7.45-7.69 (t, $\left.J=7.8 \mathrm{~Hz}, 1 \mathrm{H}, \mathrm{Ar}-\mathrm{H}\right),(\mathrm{dt}, J=1.4,7.9 \mathrm{~Hz}, 1 \mathrm{H}, \mathrm{Ar}-\mathrm{H}), 7.92$ (ddd, $J=0.8$, 2.0, 8.0 Hz, 2H, Ar-H), 8.87-8.89 (t, $J=5.6 \mathrm{~Hz}, 1 \mathrm{H}, \mathrm{Ar}-\mathrm{H})$.<smiles>COC(=O)/C=C/c1ccccc1</smiles>

Methyl trans-cinnamate Yellow solid, (17\%). ${ }^{1} \mathrm{H}$ NMR (400 MHz; $\left.\mathrm{CDCl}_{3}\right) \delta 3.83(\mathrm{~s}, 3 \mathrm{H}$, $\left.\mathrm{CH}_{3}\right), 6.70-6.72(\mathrm{~d}, J=7.6 \mathrm{~Hz}, 1 \mathrm{H}, \mathrm{CH}), 6.74-6.76(\mathrm{~d}, J=7.6 \mathrm{~Hz}, 1 \mathrm{H}, \mathrm{CH}), 7.26-7.60$ (m, $5 \mathrm{H}, \mathrm{Ar}-\mathrm{H})$. 


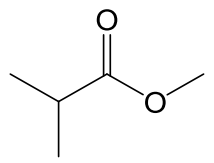

Methyl isobutyrate Colourless oil, (100\%). ${ }^{1} \mathrm{H}$ NMR (400 MHz; $\left.\mathrm{CDCl}_{3}\right) \delta 1.18-1.20(\mathrm{~d}, J=$ $5.2 \mathrm{~Hz}, 6 \mathrm{H}, \mathrm{CH}_{3}$ ), 2.57-2.62 (sept, $\left.J=6.7 \mathrm{~Hz}, 1 \mathrm{H}, \mathrm{CH}\right), 4.40\left(\mathrm{~s}, 3 \mathrm{H}, \mathrm{CH}_{3}\right)$.<smiles>COC(=O)c1ccc(OC)cc1</smiles>

Methyl anisate Yellow Oil, (5\%). ${ }^{1} \mathrm{H}$ NMR $\left(400 \mathrm{MHz} ; \mathrm{CDCl}_{3}\right) \delta 3.90\left(\mathrm{~s}, 3 \mathrm{H}, \mathrm{CH}_{3}\right), 6.52-6.53$ (dd, $1 \mathrm{H}, \mathrm{CH}), 7.18-7.19(\mathrm{dd}, J=1.6,3.6 \mathrm{~Hz}, 1 \mathrm{H}, \mathrm{CH}), 7.58-7.59\left(\mathrm{dd}, 1 \mathrm{H}, \mathrm{CH}_{3}\right)$.

\section{Procedure for the production of Sudan 1 dye}<smiles>Oc1ccc2ccccc2c1/N=N/c1ccccc1</smiles>

A solution of aniline $(0.2 \mathrm{~mL}, 0.204 \mathrm{~g}, 2.19 \mathrm{mmol})$, hydrochloric acid (10 M, $0.35 \mathrm{~mL})$, water $(2 \mathrm{~mL})$ and $\mathrm{N}, \mathrm{N}$-dimethylformamide $(4 \mathrm{~mL})$ was prepared and pumped at $0.195 \mathrm{~mL} / \mathrm{min}^{-1}$. A solution of sodium nitrite $(0.75 \mathrm{~g}, 10.9 \mathrm{mmol})$, water $(4 \mathrm{~mL})$ and $\mathrm{N}, \mathrm{N}$-dimethylformamide (2 $\mathrm{mL}$ ) was prepared and pumped at $0.195 \mathrm{~mL} / \mathrm{min}^{-1}$. The two streams intercepted at a t-piece and were pumped together at $0.39 \mathrm{~mL} / \mathrm{min}^{-1}$ giving a residence time of 30 minutes. After 30 minutes the stream was intercepted by a solution of $\beta$-naphthol $(0.3 \mathrm{~g}, 1.04 \mathrm{mmol})$, sodium hydroxide solution $(10 \%, 6 \mathrm{~mL})$, water $(3 \mathrm{~mL})$ and $\mathrm{N}, \mathrm{N}$-dimethylformamide $(3 \mathrm{~mL})$ being pumped at $0.39 \mathrm{~mL} / \mathrm{min}^{-1}$ giving a residence time of 15 minutes. Reactant products were collected and extracted using diethyl ether $(3 \times 25 \mathrm{~mL})$ and water $(25 \mathrm{~mL})$. The combined 
organic layers were dried over anhydrous magnesium sulfate and evaporated to dryness under vacuum. Samples were analysed using NMR spectroscopy. Red Solid, (17\%). ${ }^{1} \mathrm{H}$ NMR (400 MHz; $\left.\mathrm{CDCl}_{3}\right) \delta$ 6.87-6.89 (d, $\left.J=8.0,1 \mathrm{H}, \mathrm{Ar}-\mathrm{H}\right), 7.11-7.78(\mathrm{~m}, 9 \mathrm{H}, \mathrm{Ar}-\mathrm{H}), 8.56-8.58(\mathrm{~d}, J=$ $8.0 \mathrm{~Hz}, 1 \mathrm{H}, \mathrm{Ar}-\mathrm{H}), 16.28$ (s, 1H, OH).

\section{Procedure for the oxidation reaction of potassium iodide}

Potassium iodide (4.98 g, $0.03 \mathrm{mmol})$ was dissolved in $30 \mathrm{~mL}$ of water and then transferred to a small glass sample vial. To a second sample vial potassium persulfate $(8.11 \mathrm{~g}, 0.03$ mmol), sodium thiosulfate $(4.74 \mathrm{~g}, 0.03 \mathrm{mmol})$, and starch indicator (10 drops) were dissolved in $30 \mathrm{~mL}$ of water. The solutions were pumped through a T-piece at flow rates ranging from 0.6 to $1.3 \mathrm{~mL} / \mathrm{min}$, and then through both SL part 5 and a reel of PTFE tubing. The reaction progressions were timed until the characteristic dark blue colour of elemental iodine was observed.

\section{General procedure for the room temperature formation of an oxime}<smiles>O=[N+]([O-])c1ccc(/C=N/O)cc1</smiles>

For specific reagent concentrations, flow regimes and analysis methods see results and discussion section (Chapter 7.2.1, Tables 51-56). 4-Nitrobenzaldehyde was dissolved in methanol and transferred to a sample vial. To a second sample vial a solution containing hydroxylamine hydrochloride, sodium hydroxide and water was prepared. The two solutions were mixed via a T-piece, and pumped at flow rates ranging from $0.1-1 \mathrm{~mL} / \mathrm{min}$ through a number of different flow devices. The oxime product was analysed online via HPLC as well as in line via UV-Vis spectroscopy. 
General procedure for the formation of carvone semicarbazide.

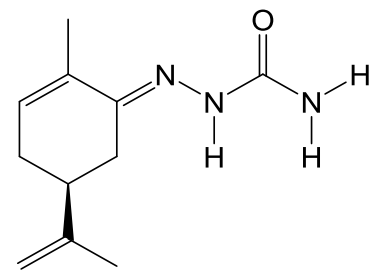

For specific reagent concentrations, flow regimes and analysis methods see results and discussion section (Chapter 7.2.2, Tables 60-69). $R-(-)$ carvone was dissolved in methanol and transferred to a sample vial. To a second sample vial a solution containing semicarbazide hydrochloride, sodium acetate and water was prepared. The two solutions were mixed via a T-piece, and pumped at flow rates ranging from $0.1-1 \mathrm{~mL} / \mathrm{min}$ through a number of different flow devices. The carvone semicarbazone product was analysed online via HPLC as well as in line via UV-Vis spectroscopy. 


\section{1. $\quad \underline{\text { References }}$}

$1 \quad$ P. Watts and C. Wiles, Chem. Commun., 2007, 5, 443-467.

2 J. Wegner, S. Ceylan and A. Kirschning, Adv. Synth. Catal., 2012, 354, 17-57.

3 P. Abgrall and A. Gué, J. Micromech. Microeng., 2007, 17, 15-49.

4 K. Jähnisch, V. Hessel, H. Löwe and M. Baerns, Angew. Chem., Int. Ed. Engl., 2004, 43, 406-446.

5 J. H. Bannock, S. H. Krishnadasan, M. Heeney and J. C. de Mello, Mater. Horiz., 2014, 4, 373-378.

6 B. H. Weigl, R. L. Bardell and C. R. Cabrera, Adv. Drug Deliv. Rev., 2003, 55, 349377.

7 V. Hessel, S. Hardt, H. Lowe and F. Schonfeld, AIChE J., 2003, 49, 566-577.

8 D. L. Browne, M. Baumann, B. H. Harji, I. R. Baxendale and S. V Ley, Org. Lett., 2011, 13, 3312-3315.

9 L. Capretto, W. Cheng, M. Hill and X. Zhang, Top. Curr. Chem, 2011, 304, 27-68.

10 C. F. Carter, H. Lange, S. V Ley, I. R. Baxendale, B. Wittkamp, J. G. Goode and N. L. Gaunt, Org. Process Res. Dev., 2010, 14, 393-404.

11 S.-A. Leung, R. F. Winkle, R. C. R. Wootton and A. J. DeMello, Anal. (Cambridge, U. K.), 2005, 130, 46-51.

12 T. Schwalbe, V. Utze and G. Wille, Chimia (Aarau)., 2002, 56, 636-646.

13 D. M. Roberge, L. Ducry, N. Bieler, P. Cretton and B. Zimmermann, Chem. Eng. Technol., 2005, 28, 318-323.

14 J. A. Newby, D. W. Blaylock, P. M. Witt, J. C. Pastre, M. K. Zacharova, S. V Ley and D. L. Browne, Org. Process Res. Dev., 2014, 18, 1211-1220.

15 Uniqsis, http://www.uniqsis.com, (accessed January 2015).

16 K. S. Elvira, X. Casadevall, R. C. R. Wootton and A. J. de Mello, Nat. Chem., 2013, 5, 905-915.

17 R. C. Wheeler, O. Benali, M. Deal, E. Farrant, S. J. F. Macdonald and B. H. Warrington, Org. Process Res. Dev., 2007, 11, 704-710.

18 B. Gutmann, J. P. Roduit, D. Roberge and C. O. Kappe, Angew. Chem., Int. Ed., 2010, 49, 7101-7105. 
19 W. R. Melchert, B. F. Reis and F. R. P. Rocha, Anal. Chim. Acta, 2012, 714, 8-19.

20 R. L. Hartman and K. F. Jensen, Lab Chip, 2009, 9, 2495-2507.

21 T. Noël, J. R. Naber, R. L. Hartman, J. P. McMullen, K. F. Jensen and S. L. Buchwald, Chem. Sci., 2011, 2, 287-290.

22 S. D. Schaber, D. I. Gerogiorgis, R. Ramachandran, J. M. B. Evans, P. I. Barton and B. L. Trout, Ind. Eng. Chem. Res., 2011, 50, 10083-10092.

23 J. S. L. Philpot, Trans. Faraday Soc., 1940, 35, 38-46.

24 A. Manz, N. Graber and H. M. Widmer, Sens. Actuators, B, 1990, 1, 244-248.

25 E. Garcia-Egido, S. Y. Wong and B. H. Warrington, Lab Chip, 2002, 2, 31-33.

26 M. A. Schmidt, R. Srinivasan, S. L. Firebaugh, I. M. Hsing, M. P. Harold and K. F. Jensen, in 9th International Conference on Solid State Sensors and Actuators, Chicago, 1997, pp. 163-166.

27 M. C. Mitchell, V. Spikmans, A. Manz and A. J. de Mello, J. Chem. Soc., Perkin Trans. 1, 2001, 5, 514-518.

28 B. Ahmed-Omer, J. C. Brandt and T. Wirth, Org. Biomol. Chem., 2007, 5, 733-740.

29 E. A. Mansur, M. Ye, Y. Wang and Y. Dai, Chin. J. Chem. Eng., 2008, 16, 503-516.

30 K. Geyer, J. Codée and P. Seeberger, Chem. - Eur. J., 2006, 12, 8434-8442.

31 I. Glasgow and N. Aubry, Lab Chip, 2003, 3, 114-120.

32 B. Pieber, S. T. Martinez, D. Cantillo and C. O. Kappe, Angew. Chem., Int. Ed., 2013, 52, 10241-10244.

33 N.-T. Nguyen and Z. Wu, J. Micromech. Microeng., 2005, 15, 1-16.

34 J. Friend and L. Yeo, Biomicrofluidics, 2010, 4, 1-5.

35 T. R. Dietrich, A. Freitag and R. Scholz, Chem. Eng. Technol., 2005, 28, 477-483.

36 A. R. Bogdan and N. W. Sach, Adv. Synth. Catal., 2009, 351, 849-854.

37 Amarequip, http://amarequip.com/, (accessed March 2016).

38 J. N. Lee, C. Park and G. M. Whitesides, Anal. Chem., 2003, 75, 6544-6554.

39 P. D. I. Fletcher, S. J. Haswell, E. Pombo-Villar, B. H. Warrington, P. Watts, Y. F. Wong and X. Zhang, Tetrahedron, 2002, 58, 4735-4757.

K. F. Jensen, MRS Bull., 2006, 31, 101-107. 
41 M. H. Yen, J. Y. Cheng, C. W. Wei, Y. C. Chuang and T. H. Young, J. Micromech. Microeng., 2006, 16, 1143-1153.

42 Micronit Microfluidics, http://store.micronit.com/foccused-flow-droplet-generatorlarge-droplets.html, (accessed January 2015).

43 P. Abgrall and A. M. Gué, J. Micromech. Microeng., 2007, 17, 15-49.

44 C. Iliescu, H. Taylor, M. Avram, J. Miao and S. Franssila, Biomicrofluidics, 2012, 6.

45 C. Iliescu, K. L. Tan, F. E. H. Tay and J. Miao, in International Conference on Materials for Advanced Technologies, 2005, pp. 75-78.

46 C.-M. Ho and Y.-C. Tai, J. Fluids Eng., 1996, 118, 437.

47 N. Blow, Nat. Methods, 2007, 4, 665-670.

48 K. a. Addae-Mensah, S. Retterer, S. R. Opalenik, D. Thomas, N. V. Lavrik and J. P. Wikswo, J. Microelectromech. Syst., 2010, 19, 64-74.

49 A. Karczemska, D. Witkowski, V. Ralchenko, A. Bolshakov, D. Sovyk, J. Lysko and J. Hassard, in Perspective Technologies and Methods in MEMS Design, 2009, pp. 2224.

50 A. Erten, M. Makale, X. Lu, B. Fruhberger, S. Kesari and S. Esener, J. Micromech. Microeng., 2011, 21.

51 IMSChips, http://ims-chips.de/, (accessed March 2016).

52 A. Berthold, L. Nicola, P. M. Sarro and M. J. Vellekoop, Sens. Actuators, A, 2000, 82, 224-228.

53 Y. J. Pan and R. J. Yang, J. Micromech. Microeng., 2006, 16, 2666-2672.

54 J. G. Bomer, A. V Prokofyev, A. Van Den Berg and S. Le Gac, Lab Chip, 2014, 14, 4461-4464.

55 J. Kim, R. Surapaneni and B. K. Gale, Lab Chip, 2009, 9, 1290-1293.

56 H. Wu, B. Huang and R. N. Zare, Lab Chip, 2005, 5, 1393-1398.

57 M. Alonso-Amigo, JALA, 2000, 5, 96-101.

58 R. A. Yadav, R. Scholar, P. S. V Joshi and A. P. N. K. Kamble, IJSER, 2012, 3, 1-8.

59 HicksPlastic, http://hicksplastic.com/, (accessed March 2016).

60 U. M. Attia, S. Marson and J. R. Alcock, Microfluid. Nanofluid., 2009, 7, 1-28. 
61 H. Becker, W. Dietz, P. Dannberg and F. Institut, in Micro Total Analysis Systems, 1998, pp. 253-256.

62 S. J. Lee and N. Sundararajan, Microfabrication for Microfluidics, Springer, New York, 2010.

63 J. J. Brandner, in Microreactors in Organic Synthesis and Catalysis, ed. T. Wirth, Wiley, Hoboken, 2008, pp. 1-15.

64 P. Risthaus, J. Haubelt, R. Knitter and D. Gohring, Microsyst. Technol., 2001, 7, 8590.

65 C. Chan, G. Cao and T. Stoebe, Microsyst. Technol., 2000, 6, 200-204.

66 B. Mutsuddy and R. Ford, Ceramic Injection Molding, Chapman and Hall, London, 1995.

67 I. Peltsman and M. Peltsman, in Cermamic Engineering and Science Proceedings, 1981, vol. 2, pp. 1006-1010.

68 B. R. Knitter, W. Bauer and D. Göhring, Adv. Eng. Mater., 2001, 3, 49-54.

69 Z. S. Rak, Powder Met. Met. Ceram., 1999, 38, 126-132.

70 D. J. Beebe, G. A. Mensing and G. M. Walker, Annu. Rev. Biomed. Eng., 2002, 4, 261-86.

71 P. Gravesen, J. Branebjerg and O. S. Jensen, J. Micromech. Microeng., 1993, 3, 168182.

72 T. M. Squires, Rev. Mod. Phys., 2005, 77, 977-1026.

73 L. Capretto, W. Cheng, M. Hill and X. Zhang, Top. Curr. Chem., 2011, 304, 27-68.

74 N. Schwesinger, T. Frank and H. Wurmus, J. Micromech. Microeng., 1996, 6, 99-102.

75 J. B. Knight, A. Vishwanath, J. P. Brody and R. H. Austin, Phys. Rev. Lett., 1998, 80, 3863-3866.

76 A. Gu, M. Jhunjhunwala, M. Thalmann, M. A. Schmidt and K. F. Jensen, Langmuir, 2005, 21, 1547-1555.

77 H. Song, J. D. Tice and R. F. Ismagilov, Angew. Chem., 2003, 42, 768-772.

78 V. Hessel, H. Löwe and F. Schönfeld, Chem. Eng. Sci., 2005, 60, 2479-2501.

79 D. Gobby, P. Angeli and A. Gavriilidis, J. Micromech. Microeng., 2001, 11, 126-132.

80 T. J. Johnson, D. Ross and L. E. Locascio, Anal. Chem., 2002, 74, 45-51. 
81 S. Wong, M. Ward and C. Wharton, Sens. Actuators, B, 2004, 100, 359-379.

82 T. T. Veenstra, T. S. J. Lammerink, M. C. Elwenspoek and A. van den Berg, J. Micromech. Microeng., 1999, 9, 199-202.

83 S. W. Lee, D. S. Kim, S. S. Lee and T. H. Kwon, J. Micromech. Microeng., 2006, 16, 1067-1072.

84 A. D. Stroock, S. K. W. Dertinger, A. Ajdari, I. Mezic, H. A. Stone and G. M. Whitesides, Science (80-. )., 2002, 295, 647-651.

85 Y. Lin, G. J. Gerfen, D. L. Rousseau and S. R. Yeh, Anal. Chem., 2003, 75, 53815386.

86 V. Mengeaud, J. Josserand and H. H. Girault, Anal. Chem., 2002, 74, 4279-4286.

87 R. A. Vijayendran, K. M. Motsegood, D. J. Beebe and D. E. Leckband, Langmuir, 2003, 19, 1824-1828.

88 A. Günther and K. F. Jensen, Lab Chip, 2006, 6, 1487-1503.

89 H. Gu, M. H. G. Duits and F. Mugele, Int. J. Mol Sci., 2011, 12, 2572-2597.

90 G. G. Yaralioglu, I. O. Wygant, T. C. Marentis and B. T. Khuri-Yakub, Anal. Chem., 2004, 76, 3694-3698.

91 I. Glasgow and N. Aubry, Lab Chip, 2003, 3, 114-120.

92 Z. Yang, S. Matsumoto, H. Goto, M. Matsumoto and R. Maeda, Sens. Actuators, A, 2001, 93, 266-272.

93 J. H. Tsai and L. Lin, Sens. Actuators, A, 2002, 97-98, 665-671.

94 P. Paik, V. K. Pamula, M. G. Pollack and R. B. Fair, Lab Chip, 2003, 3, 28-33.

95 H. H. Bau, Sens. Actuators, A, 2001, 79, 207-215.

96 A. A. Deshmukh, D. Liepmann and A. P. Pisano, in IEEE solid-state sensor and actuator workshop, 2009, pp. 73-76.

97 M. H. Oddy, J. G. Santiago and J. C. Mikkelsen, Anal. Chem., 2001, 73, 5822-5832.

98 H. Y. Lee and J. Voldman, Anal. Chem., 2007, 79, 1833-1839.

99 L. H. Lu, K. S. Ryu and C. Liu, J. Microelectromech. Syst., 2002, 11, 462-469.

100 K. F. Jensen, in Microreactor Technology and Process Intensification, eds. Y. Wong and J. D. Holladay, American Chemical Society, Washington DC, 2005, pp. 1-22. 
101 J. Yue, J. C. Schouten and T. A. Nijhuis, Ind. Eng. Chem. Res., 2012, 51, 1458314609.

102 P. A. Auroux, D. Iossifidis, D. R. Reyes and A. Manz, Anal. Chem., 2002, 74, 26372652.

103 M. Kakuta, P. Hinsmann, A. Manz and B. Lendl, Lab Chip, 2003, 3, 82-85.

104 R. Herzig-Marx, K. T. Queeney, R. J. Jackman, M. A. Schmidt and K. F. Jensen, Anal. Chem., 2004, 76, 6476-6483.

105 C. Liu, D. Cui and X. Chen, J. Chromatogr. A, 2007, 1170, 101-106.

106 A. Cleary, S. García-Blanco, A. Glidle, J. S. Aitchison, P. Laybourn and J. M. Cooper, IEEE Sens. J., 2005, 5, 1315-1320.

107 P. Hinsmann, J. Frank, P. Svasek, M. Harasek and B. Lendl, Lab Chip, 2001, 1, 16-21.

108 T. M. Floyd, M. A. Schmidt and K. F. Jensen, Ind. Eng. Chem. Res., 2005, 44, 23512358.

109 P. J. Viskari and J. P. Landers, Electrophoresis, 2006, 27, 1797-1810.

110 W. Ferstl, T. Klahn, W. Schweikert, G. Billeb, M. Schwarzer and S. Loebbecke, Chem. Eng. Technol., 2007, 30, 370-378.

111 Y. Takahashi, M. Nakakoshi, S. Sakurai, Y. Akiyama, H. Suematsu, H. Utsumi and T. Kitamori, Anal. Sci., 2007, 23, 395-400.

112 Vapourtec, http://vapourtec.com/, (accessed January 2016).

113 http://www.astm.org/COMMITTEE/F42.htm/, (accessed January 2016).

114 R. D. Goodridge, C. Ohtsuki, M. Kamitakahara, D. J. Wood and K. W. Dalgarno, Key Eng. Mater., 2006, 309-311, 289-292.

115 M. Stern, G. Franchin, M. Kayser, J. Klein, C. Inamura, S. Dave, J. C. Weaver, P. Houk, P. Colombo, M. Yang and N. Oxman, 3D Print. Addit. Manuf., 2015, 2, 92105.

116 D. Therriault, S. R. White and J. a Lewis, Nat. Mater., 2003, 2, 265-71.

117 J. Bruneaux, D. Therriault and M. C. Heuzey, J. Micromech. Microeng., 2008, 18, 111.

118 M. D. Symes, P. J. Kitson, J. Yan, C. J. Richmond, G. J. T. Cooper, R. W. Bowman, T. Vilbrandt and L. Cronin, Nat. Chem., 2012, 1-6.

119 P. J. Kitson, M. H. Rosnes, V. Sans, V. Dragone and L. Cronin, Lab Chip, 2012, 12, 3267-3271. 
120 3D Touch, http://www.3dsystems.com/shop/support/legacy/supplies, (accessed January 2015).

121 J. S. Mathieson, M. H. Rosnes, V. Sans, P. J. Kitson and L. Cronin, Beilstein J. Nanotechnol., 2013, 4, 285-91.

122 V. Dragone, V. Sans, M. H. Rosnes, P. J. Kitson and L. Cronin, Beilstein J. Org. Chem., 2013, 9, 951-959.

123 P. J. Kitson, M. D. Symes, V. Dragone and L. Cronin, Chem. Sci., 2013, 4, 3-7.

124 G. Chisholm, P. J. Kitson, N. D. Kirkaldy, L. G. Bloor and L. Cronin, Energy Environ. Sci., 2014, 7, 3026-3032.

125 K. B. Anderson, S. Y. Lockwood, R. S. Martin and D. M. Spence, Anal. Chem., 2013, 85, 5622-5626.

126 J. L. Erkal, A. Selimovic, B. C. Gross, S. Y. Lockwood, E. L. Walton, S. McNamara, R. S. Martin and D. M. Spence, Lab Chip, 2014, 14, 2023-32.

127 J. L. Erkal, A. Selimovic, B. C. Gross, S. Y. Lockwood, E. L. Walton, S. McNamara, R. S. Martin and D. M. Spence, Lab Chiphip, 2014, 14, 2023-32.

128 K. C. Bhargava, B. Thompson and N. Malmstadt, Proc. Natl. Acad. Sci. U. S. A., 2014, 111, 15013-15018.

129 3DSystems, http://www.3dsystems.com/3d-printers/professional/projet-3500-hdmax, (accessed January 2015).

130 A. K. Au, W. Lee and A. Folch, Lab Chip, 2014, 14, 1294-301.

131 3DSystems, http://www.3dsystems.com, (accessed January 2015).

132 P. Risthaus, J. Haubelt, R. Knitter and D. Gohring, Microsyst. Technol., 2001, 7, 8590.

133 C. Martino, S. Berger, R. C. R. Wootton and J. Andrew, Lab Chip, 2014, 14, 41784182.

134 L. Novakova-Marcincinova, Manuf. Ind. Eng., 2012, 11, 35-37.

135 R. D. Goodridge, R. J. M. Hague and C. J. Tuck, J. Mater. Process. Technol., 2010, 210, 72-80.

136 F. P. W. Melchels, J. Feijen and D. W. Grijpma, Biomaterials, 2010, 31, 6121-30.

137 R. Hague, S. Mansour, N. Saleh and R. Harris, J. Mater. Sci., 2004, 39, 2457-2464. 
138 K. Altaf, I. A. Ashcroft and R. Hague, in Proceedings of the 20th International Workshop on Computational Mechanics of Materials - IWCMM 20, 2012, pp. 112117.

139 B. Sager, D. W. Rosen, M. Shilling and T. R. Kurfess, in Solid Freeform Fabrication, 2003, pp. 70-81.

140 DSM, http://www.dsm.com/products/somos/en_US/home.html, (accessed January 2015).

141 K. Altaf, I. A. Ashcroft and R. Hague, Comput. Mater. Sci., 2012, 52, 112-117.

142 3DSystems, http://www.3dsystems.com/, (accessed January 2015).

143 CustomPart, http://www.custompartnet.com, (accessed January 2015).

144 A. B. Leduc and T. F. Jamison, Org. Process Res. Dev., 2012, 16, 1082-1089.

145 R. C. Wootton, R. Fortt and A. J. de Mello, Lab Chip, 2002, 2, 5-7.

146 R. C. R. Wootton, R. Fortt and A. J. De Mello, Lab Chip, 2002, 2, 5-7.

147 V. Hessel, F. Schonfeld and C. Hofmann, Lab Chip, 2004, 65-69.

148 A. E. Burgess and J. C. Davidson, J.Chem.Educ, 2012, 89, 814-816.

149 S. Lawton, G. Steele, P. Shering, A. Engineering, A. Park, C. Sk, L. Zhao and I. Laird, Org. Process Res. Dev., 2009, 13, 1357-1363.

150 D. Freitag, T. Wohlers and T. Philippi, J. Mater. Process. Technol., 2004, 149, 616622.

151 M. Rombouts, L. Froyen, P. Mercelis and J. P. Kruth, CIRP Ann., 2006, 55, 187-192.

152 S. Pauly, L. Löber, R. Petters, M. Stoica, S. Scudino, U. Kühn and J. Eckert, Mater. Today, 2013, 16, 37-41.

153 L. Thijs, F. Verhaeghe, T. Craeghs, J. Van Humbeeck and J. P. Kruth, Acta Mater., 2010, 58, 3303-3312.

154 B. Vayre, F. Vignat and F. Villeneuve, Mech. Ind., 2012, 13, 89-96.

155 ExtremeTech, http://www.extremetech.com, (accessed January 2015).

156 I. Yadroitsev and I. Smurov, Phys. Procedia, 2011, 12, 264-270.

157 L. Papadakis, A. Loizou, J. Risse and J. Schrage, in Procedia CIRP, 2014, vol. III, pp. 90-95.

158 Renishaw, http://www.renishaw.com, (accessed January 2015). 
159 D. E. Long, Anal. Chim. Acta, 1969, 46, 193-206.

160 E. Seifert, L. Abramo, B. Thelin, T. Lundstedt, A. Nystrom, J. Petteresen and R. Bergman, Chemom. Intell. Lab. Syst., 1998, 42, 3-40.

161 D. M. Roberge, Org.Proc.Res.Dev, 2004, 8, 1049-1053.

162 K. Koch, B. J. A. Van Weerdenburg, J. M. M. Verkade, P. J. Nieuwland, F. P. J. T. Rutjes and J. C. M. Van Hest, Org. Process Res. Dev., 2009, 13, 1003-1006.

163 J. A. Palasota and S. N. Deming, J. Chem. Educ., 1992, 69, 560-563.

164 C. R. T. Tarley, G. Silveira, W. N. L. dos Santos, G. D. Matos, E. G. P. da Silva, M. A. Bezerra, M. Miró and S. L. C. Ferreira, Microchem. J., 2009, 92, 58-67.

165 R. D. Krause and J. A. Lott, Clin. Chem., 1974, 20, 775-782.

166 S. N. Deming, M. L. Parsons and C. L. Shavers, J. Chem. Educ., 1979, 56, 307-309.

167 F. H. Walters, L. R. Parker Jr., S. L. Morgan and S. N. Deming, Sequential Simplex Optimization, CRC Press, Boca Raton, 1991.

168 J. P. Mcmullen, M. T. Stone, S. L. Buchwald and K. F. Jensen, Angew. Chem., 2010, 49, 7076-7080.

169 J. R. Hyde, R. A. Bourne, I. Noda, P. Stephenson and M. Poliakoff, Anal. Chem., 2004, 76, 6197-6206.

170 R. A. Bourne, R. A. Skilton, A. J. Parrott, D. J. Irvine and M. Poliako, Org.Proc.Res.Dev, 2011, 15, 932-938.

171 R. A. Skilton, A. J. Parrott, M. W. George, M. Poliakoff and R. A. Bourne, Appl. Spectrosc., 2013, 67, 1127-31.

172 E. V. Gurkovskii, V. K. Leko and I. A. Stepanova, Steklo. Keram., 1974, 15-16.

173 R. J. Friel and R. A. Harris, Procedia CIRP, 2013, 6, 35-40.

174 C. Y. Kong and R. Soar, Appl. Opt., 2005, 44, 6325-6333.

175 T. Monaghan, A. J. Capel, S. D. Christie, R. A. Harris and R. J. Friel, Compos. Part A, 2015, 76, 181-193. 


\section{Appendix}

\subsection{Design of a Simplex Reaction Optimisation Model}

\subsection{Introduction}

In order to perform both inline and online optimisation of the reactions, as well as having complete control over reaction residence times, temperatures, and reagent compositions, it was necessary to design a series of macros which would allow the control over each component of an Agilent LC system. In order to do this it was necessary to be able to control the Chemstation software that is the graphical user interface (GUI) for the LC system. MacroPad is a bespoke piece of macro development software specifically designed for developing macros which are capable of controlling the Chemstation software. Through MacroPad, it is possible to access the Chemstation registers, which store all the input and output data produced during the LC analysis. The information stored in these registers will allow complete control over both the LC input variables such as flow rates, temperatures and pressures, as well as quantitative outputs such as spectroscopic and chromatographic data from any LC analysis which has been run. It was therefore proposed that MacroPad would be used to code a series of bespoke macros, which could be run through Chemstation allowing us to intimately control the reaction and LC conditions.

The control and command of the LC software would need to be coupled with a separate piece of software which would run the simplex algorithms. This programme was generated using the Visual Basics (VB) programming language built into Microsoft Excel. VB was selected as the programming language because it is simple to use, with a large network of internet support forums. The GUI of Excel is also familiar to most people, allowing the software to be user-friendly to anyone unfamiliar with reaction optimisation, as well as allowing them to easily process any data generated. Using VB would also not require the installation of any new software onto the computers running the department LC systems, allowing the simple transportation of the programme between computers on a data storage device. 


\subsection{Results and Discussion}

\subsection{Chemstation Macros}

The following is a description of the function of all the macros that were written, and how they operated within the Chemstation software. There will not be references to specific lines of code, however an annotated copy of this is attached to this document on a separate CD.

Having opened the Chemstation software, the user must load the optimisation macros using the Chemstation dialog input box. The user must then create a template method, which defines the initial reaction and analysis conditions for the pumping modules, column compartment, DAD compartment, as well as the external 6 port valve. The 6 port valve is programmed to inject a slug of reaction medium onto the column at the beginning of each new LC method. This template can be saved and re-opened for future run sequences. This method will define the reaction and analysis conditions which each of the optimisation runs will be based around. For the "Create Optimisation Set" tab the user must generate a .txt file containing the set of reaction conditions which are to be run.

Upon loading of the Chemstation macros, a new drop down menu called "Reaction Monitoring" will be available to the user. This drop down menu allows the user to either select the "Create Optimisation Set" or the "Automation" option on the menu. These two features have slightly different functions. 


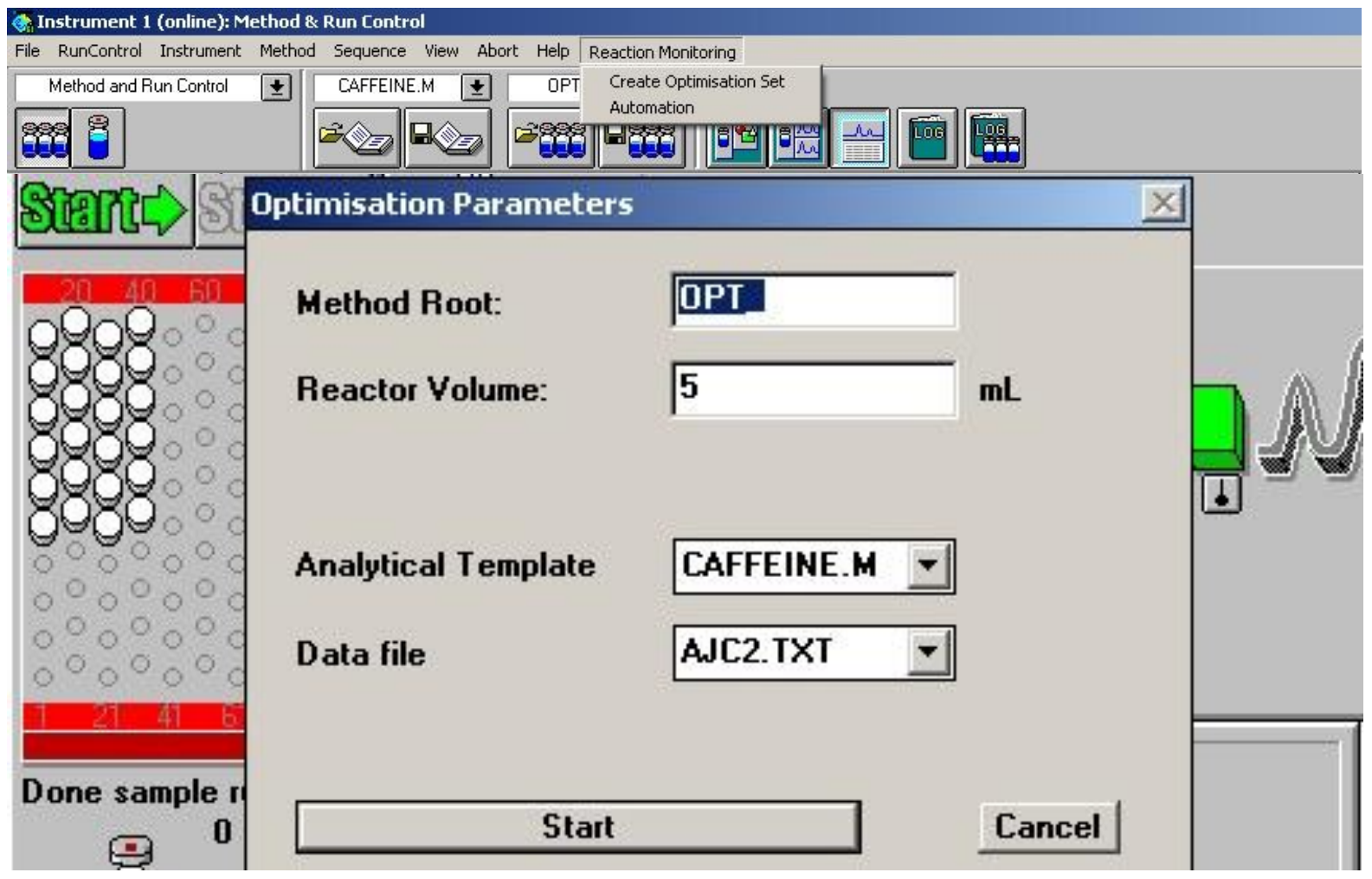

Figure 122: Examples of the reaction optimisation user interface macro within Chemstation.

\section{Create Optimisation Set}

The "Create Optimisation Set" tab allows the user to create a non-automated optimisation set by manually inputting each new set of reaction conditions into the LC software. Upon selecting the "Create Optimisation Set" tab any data previously stored in the Chemstation registers is refreshed, and the user is presented with an input dialog box. This dialog box allows the user to define the reactor volume, the data set save location, the template method to be used, and the location of the input .txt data file. The user can then select the start button which generates the new reaction conditions (based on the reaction conditions outlined within the .txt file previously generated). This method is added to a sequence which defines each of the individual reaction conditions which have been or will be run within the new optimisation set. Once the new set of conditions have been run, the user can manually analyse the data generated and determine the next set of conditions they wish to run in the optimisation sequence.

\section{$\underline{\text { Automation }}$}

The "Automation" tab allows the user to create a fully automated optimisation set, whereby the Chemstation and visual macros control the entire optimisation. Again, having selecting 
the "Automation" tab any data previously stored in the Chemstation registers is refreshed, and the user is presented with an input dialog box. This dialog box allows the user to define the reactor volume, the data set save location, the template method to be used, and the location of the input .txt data file. This time however, when the user selects the start tab the software takes over complete control of the optimisation, which will continue to run until predefined stopping criteria are reached. Selection of this tab loads a second macro (AC_S_Main) which opens and reads the data within the .txt file. If there is no text file available, the macro will wait until one appears. A third macro (AC_S_CreateMethod) is loaded and the .txt file is deleted. The CreateMethod macro defines the new LC method settings including the reaction flow rate, reaction temperature and reagent composition. The new method is then saved with a unique name corresponding to its individual experiment number. The macro will then calculate the reaction residence time from the new flow rate and the previously inputted reactor volume. This residence time is inputted into the method as a "post-time" delay, which gives the new reaction conditions time to equilibrate before a slug of the flow is injected onto the column. The new method conditions are saved and a fourth macro (AC_AddToSequence) is loaded. The add sequence macro creates a new row within the current sequence and adds the existing method to that row. This sequence is saved and once the equilibration delay period has been completed the new method will be run. Once the LC analysis for the run has been completed, a post run macro (AC_S_FindProduct) is loaded. Various iterations of this macro were produced, altering the data outputted within the .txt file. These iterations included data output as reaction yield, peak intensity, and peak area. This data is then output as another .txt file, which will be read either manually or by an external piece of optimisation software. The method then reloads AC_Main and the process repeats itself. This loop will continue until no more .txt files are available to be read.

\subsection{Visual Basics Simplex Model}

Having generated the macros which would allow us control over the reaction conditions and LC analysis for each specific reaction, next a programme which defined a flexible simplex model must be generated. This model must be able to input LC analytical data from a .txt file, generate a new set of reactions conditions, and output these conditions as a new .txt file. It was initially decided that a two variable simplex model (temperature and flow rate) could be generated, before moving on to a more complex three variable design. The programme 
generated had a Microsoft Excel worksheet user interface, whereby the user could define the specific simplex model as well as the initial reaction conditions. This allows them to specify the starting reaction temperature, flow rate and reagent composition for the first experiment. It also allows them to define the size of the initial simplex as a function of the variation in temperture and flow rate between each point. The user can also specify the limits of the simplex plot, in terms of the minimal and maximal allowable tempertures and flow rates, as well as defining the maximum number of experiments to run for each optimisation. Once this data has been inputted the user can select the Reset Data tab to clear the spreadsheet of any previous data runs and select begin analysis. This will generate the txt file containing the initial reaction conditions, which will be read by the Chemsation optimisation macros. The programme will then wait until a new .txt file has been generated by Chemstation containing the data output from that run.

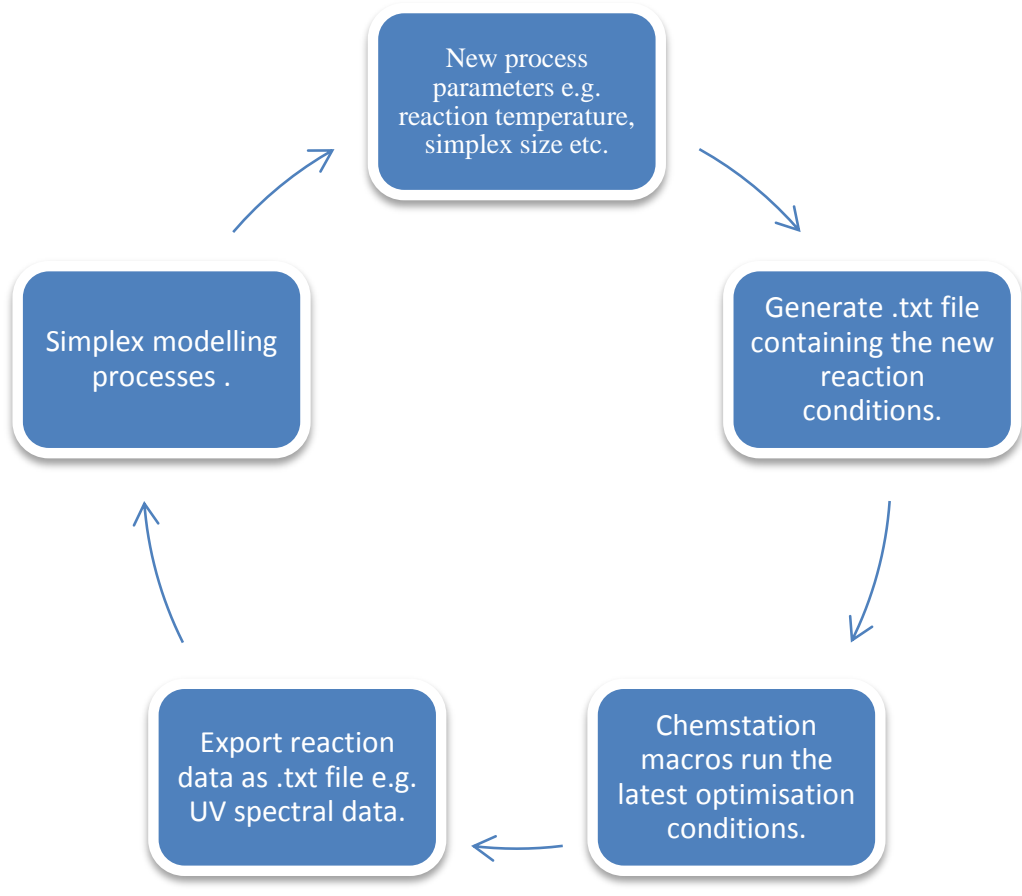

Figure 123: The VB simplex model used during the reaction optimisation process. 


\begin{tabular}{|c|c|c|c|c|}
\hline 4 & B & c & D & E \\
\hline \multicolumn{5}{|l|}{1} \\
\hline 2 & Start Temperature & 30.0 & ${ }^{\circ} \mathrm{C}$ & \\
\hline \multicolumn{5}{|c|}{ 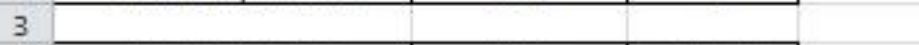 } \\
\hline 4 & Start Flow Rate & 0.90 & $\mathrm{~mL} / \mathrm{min}$ & \\
\hline \multicolumn{5}{|l|}{5} \\
\hline 6 & Start \% B & 50.0 & $\%$ & \\
\hline \multicolumn{5}{|l|}{7} \\
\hline 8 & Temperature Variable & 5.0 & ${ }^{\circ} \mathrm{C}$ & \\
\hline \multicolumn{5}{|l|}{9} \\
\hline 10 & Flow Rate Variable & 0.10 & $\mathrm{~mL} / \mathrm{min}$ & \\
\hline \multicolumn{5}{|l|}{11} \\
\hline 12 & Max Data Points & 30 & & \\
\hline 13 & Current Data Points & 25 & & \\
\hline 14 & & Area & Temp & Flow \\
\hline 15 & Old Point 1 & 0 & 0.0 & 0.00 \\
\hline 16 & Old Point 2 & 0 & 0.0 & 0.00 \\
\hline 17 & Worst Point 3 & 0 & 0.0 & 0.00 \\
\hline \multicolumn{5}{|l|}{18} \\
\hline 19 & New Data Point & 0 & 0.0 & 0.00 \\
\hline 20 & Deleted Data Point & 0 & 0.0 & 0.00 \\
\hline 21 & Triangulated Point & 0 & & \\
\hline 22 & Recalculation Point & 0 & 0.0 & 0.00 \\
\hline 23 & Secondary Deleted Point & 0 & 0.0 & 0.00 \\
\hline \multicolumn{5}{|l|}{24} \\
\hline \multicolumn{5}{|l|}{25} \\
\hline 26 & Reset Data & \multirow{2}{*}{\multicolumn{2}{|c|}{ Begin Analysis }} & \\
\hline \multirow{2}{*}{\multicolumn{5}{|c|}{28}} \\
\hline & & & & \\
\hline 29 & 26 & & 0.44 & 27.5 \\
\hline \multicolumn{5}{|l|}{30} \\
\hline 31 & Temperature High & 80 & & \\
\hline 32 & Temperature Low & 25 & & \\
\hline 33 & Flow High & 1 & & \\
\hline 34 & Flow Low & 0.2 & & \\
\hline
\end{tabular}

Figure 124: User interface of simplex programme allowing the user to input the initial reaction conditions.

Once the LC analysis for that reaction has been completed and Chemstation has outputted a .txt file, the programme will open, read and transfer the data into the data collection spreadsheet. This spreadsheet saves all the reaction conditions and LC outputs as well as specifying the data points currently part of the current simplex. It also displays the points which have been run in a $2 \mathrm{D}$ graph to allow the user to visualise the optimisation. The simplex algorithm initially coded was similar to the standard model described in section 7.1, however a couple of alterations were made. The initial simplex was not generated as a set of 3 data points simultaneously. Instead it was decided to generate each point of the simplex individually, with each new point being calculated upon the determination of the previous points data input. Secondly, when the simplex passed outside of the limits of the data set, the programme simply generated a new point randomly within the plot, instead of applying an arbitrarily low value to this point and continuing the simplex by removing the second worst 
point from the data set. Both of these alterations were made to initially simplify the coding of the programme, and future iterations of the programme would look to integrate these features into the design.

\begin{tabular}{|c|c|c|c|c|c|c|c|}
\hline Data Point & Temp & Flow & $\%$ B & Yield & Active & Matching & Data Point \\
\hline 1 & 30.00 & 0.90 & 50.0 & 8.32636 & 0 & 0 & 1 \\
\hline 1 & 35 & 0.80 & 50.0 & 10.6324 & 0 & 0 & 2 \\
\hline 1 & 30.0 & 0.70 & 50.0 & 11.3887 & 0 & 0 & 3 \\
\hline 1 & 35 & 0.6 & 50.0 & 13.8188 & 0 & 0 & 4 \\
\hline 1 & 30 & 0.5 & 50.0 & 14.5417 & 0 & 0 & 5 \\
\hline 1 & 35 & 0.4 & 50.0 & 19.988 & 0 & 0 & 6 \\
\hline 1 & 30.0 & 0.30 & 50.0 & 25.0282 & 0 & 0 & 7 \\
\hline 1 & 35 & 0.2 & 50.0 & 31.0949 & 0 & 0 & 8 \\
\hline 1 & 78.3 & 0.67 & 50.0 & 44.3399 & 0 & 0 & 9 \\
\hline 1 & 34.8 & 0.28 & 50.0 & 27.0801 & 0 & 0 & 10 \\
\hline 1 & 39.8 & 0.38 & 50.0 & 28.5246 & 0 & 0 & 11 \\
\hline 1 & 34.8 & 0.48 & 50.0 & 22.2377 & 0 & 0 & 12 \\
\hline 1 & 27 & 0.55 & 50.0 & 17.987 & 0 & 0 & 13 \\
\hline 1 & 74.6 & 0.44 & 50.0 & 49.136 & 0 & 0 & 14 \\
\hline 1 & 69.6 & 0.34 & 50.0 & 54.4903 & 0 & 0 & 15 \\
\hline 1 & 64.6 & 0.44 & 50.0 & 48.835 & 0 & 0 & 16 \\
\hline 1 & 79.6 & 0.34 & 50.0 & 52.7886 & 0 & 0 & 17 \\
\hline 1 & 74.6 & 0.24 & 50.0 & 62.6701 & 0 & 0 & 18 \\
\hline 1 & 64.6 & 0.24 & 50.0 & 58.55 & 0 & 0 & 19 \\
\hline 1 & 50.1 & 0.82 & 50.0 & 23.7797 & 0 & 0 & 20 \\
\hline 1 & 55.1 & 0.72 & 50.0 & 27.5764 & 0 & 0 & 21 \\
\hline 1 & 60.1 & 0.82 & 50.0 & 37.4671 & 0 & 0 & 22 \\
\hline 1 & 65.1 & 0.72 & 50.0 & 43.7282 & 0 & 0 & 23 \\
\hline 1 & 70.1 & 0.82 & 50.0 & 33.6152 & 0 & 0 & 24 \\
\hline 1 & 70.1 & 0.62 & 50.0 & 25.7608 & 0 & 0 & 25 \\
\hline 0 & 27.5 & 0.44 & & & 1 & 0 & 26 \\
\hline 0 & & & & & 0 & 0 & \\
\hline & & & & & & & \\
\hline
\end{tabular}

Figure 125: Data storage section of simplex programme allowing the user to visualise all reaction conditions run.

The programme would continue generating new data points until the data point limit had been met. Having generated all the required data, the programme would then transfer the data set into a separate spreadsheet and convert it into a 3D scatter plot to allow simple visualisation of the data. A separate iteration of this programme was also developed, which would allow temperature data inputted from an embedded thermocouple within the reactor (Chapter 9).

\subsection{Conclusion}

If $\mathrm{AM}$ processes are to achieve their potential of manufacturing fluidic devices with a range of integrated inline analysis tools, then chemists will need to develop a range of robust analytical software packages and instruments capable of interpreting and analysing this data in a time and cost effective manner. Software packages, such as the one developed during the course of this research, which integrate AM fluidic devices with pre-existing flow and analytical equipment will be highly sought after, due to the reduction in time and expenditure required to integrate them into daily laboratory processes. 


\subsection{Future Work}

Future work will focus on developing a more complex simplex model capable of dealing with multiple input variables, as well as a range of output variables. This will also include developing macros capable of working with a wide range of typical flow and analytical instrumentation, allowing the optimisation software to be applied to a number of applications. The model could also be developed so that it is capable of dealing with points outside of the allowable data range, as well as analysing a more precise area of the data set once an optimum has been found, to pinpoint the exact maximal output. 


\subsection{Publications List}

A. J. Capel, S. Edmondson, S.D.R. Christie, R.D. Goodridge, R.J. Bibb and M. Thurstans, Lab Chip, 2013, 13, 4583-4590.

T. Monaghan, A.J. Capel, S.D.R. Christie, R.A. Harris, R.J. Friel, Composites Part A, 2015, 76, 181-193.

\subsection{Videos}

Attached to this thesis on a separate memory stick are videos of some of the AM manufacturing processes described in this thesis. These videos are included with the aim of supplementing the descriptive process. These videos are titled as follows;

- Fused Deposition Modelling (FDM) Process at Loughborough University

- Selective Laser Sintering (SLS) Process at Loughborough University

- Stereolithography (SL) Process at Loughborough University

\subsection{Optimisation Macros}

Attached to this thesis on a separate memory stick are annotated versions of the Chemstation and Visual Basics macros used during the LC reaction optimisation detailed in this thesis. 